\title{
A Structural Framework for Assembly Modeling and Recognition
}

Christian Bauckhage 
Dipl.-Inform. Christian Bauckhage

AG Angewandte Informatik

Technische Fakultät

Universität Bielefeld

email: cbauckha@techfak.uni-bielefeld.de

Abdruck der genehmigten Dissertation zur Erlangung des akademischen Grades Doktor-Ingenieur (Dr.-Ing.).

Der Technischen Fakultät der Universität Bielefeld

am 25.2.2002 vorgelegt von Christian Bauckhage.

Gutachter:

Prof. Dr. Gerhard Sagerer

Prof. Dr. Horst Bunke

Prüfungsausschuss:

Prof. Dr. Helge Ritter

Prof. Dr. Gerhard Sagerer

Prof. Dr. Horst Bunke

Dr. Bernhard Jung

Gedruckt auf alterungsbeständigem Papier nach ISO 9706 


\section{A Structural Framework for}

\section{Assembly Modeling and Recognition}

Der Technischen Fakultät der Universität Bielefeld

zur Erlangung des Grades

Doktor-Ingenieur

vorgelegt von

Christian Bauckhage

Bielefeld - Februar 2002 

To my parents 



\section{Acknowledgments}

Writing a thesis in computer science while being employed in a collaborative research center is not about barricading oneself in an office and leaving it a couple of years later with thousands of lines of fabulous code and a book. Rather, it means to discuss ideas and approaches, to co-develop algorithms and datastructures, and to coauthor scientific papers. Consequently, there are many people whose support and cooperation throughout my PhD years I appreciate wholeheartedly.

First of all, I would like to thank my advisor Gerhard Sagerer who continuously supported and encouraged me and trusted me to find my own approach to the subject treated in this thesis. In particular, I want to thank him for giving me the opportunity to meet people and present my work on many conferences even while I was still a $\mathrm{PhD}$ student. Also, I am very grateful to Horst Bunke whose books on syntactic and structural methods in computer vision were a valuable source of insight and ideas and most positively influenced my own work. Furthermore, not only did he agree to review this thesis but despite his tight schedule also found the time to attend my disputation.

Then of course, I want to express my gratitude to the members of the Applied Computer Science Group who were just great colleagues. As they are many, I cannot mention everyone but let me single out a few: Franz Kummert always knew a way out when I was lost in the labyrinth that programming in ERNEST can become to the novice. Gernot Fink provided many, many useful suggestions when it finally came to writing down this thesis and Frank Lömker always appeared to be a walking encyclopedia when I had unsolvable technical problems. With Jannik Fritsch, Elke Braun, and Sven Wachsmuth I had a lot of fruitful discussions and they inspired and initiated many extensions of my programs which I would never have come up with by myself. Susanne Kronenberg introduced me to the science and phenomena of discourse and Britta Wrede and Christoph Schillo created the most pleasant atmosphere one can wish for in an office. Also, I deeply appreciate the assistance of Lisabeth van Iersel who always lent me a helping hand when there was bureaucratic paperwork.

Finally, but not at all in the least, I would like to thank my family, i.e. my parents and my brother, and Miriam for their assistance, affection, and emotional support. Without them, I would never have come this far. 



\section{Contents}

1. Introduction 1

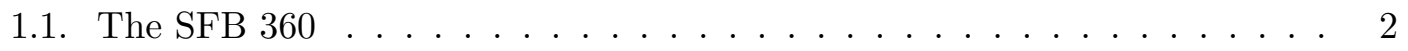

1.2. Aspects of Assemblies in Cooperative Construction . . . . . . . . . . . 3

1.3. Assemblies and Computer Vision . . . . . . . . . . . . . . . . 6

1.4. Organization of this Thesis . . . . . . . . . . . . . 8

2. Assembly Modeling and Monitoring $\quad 11$

2.1. Definition of Mechanical Assemblies . . . . . . . . . . . . . . . . . . . 12

2.2. Geometrical Assembly Modeling . . . . . . . . . . . . . . . . . . . . . 13

2.3. Structural Assembly Modeling . . . . . . . . . . . . . . . . . . . 14

2.4. Assembly Modeling within the SFB $360 \ldots \ldots$. . . . . . . . . 18

2.5. Visual Assembly Process Monitoring . . . . . . . . . . . . . . . . . . . . 20

2.6. Mathematical Complexity . . . . . . . . . . . . . . . . . 22

2.7. Discussion . . . . . . . . . . . . . . . . . . . . 23

3. Syntactic Assembly Modeling 27

3.1. Formal Language Theory and Assembly Modeling . . . . . . . . . . . . . 30

3.1.1. A Context Free Grammar of baufix ${ }^{\circledR}$ Assemblies . . . . . . . . . . . 31

3.1.2. Learning Assembly Grammars from Examples . . . . . . . . . . . 32

3.2. Syntactic Approaches to Assembly Detection . . . . . . . . . . . . . . . . 40

3.2.1. Semantic Networks as Parsers . . . . . . . . . . . . . . . . . 41

3.2.2. Assembly Parsing and Discourse Theory . . . . . . . . . . . . 44

3.3. Spinoffs from Syntactic Assembly Detection . . . . . . . . . . . . . . . . 50

3.3.1. Learning Assembly Sequence Plans from Vision . . . . . . . . . 50

3.3.2. Combining Assembly and Action Detection . . . . . . . . . . . . 54

3.3.3. Application of Syntactic Context . . . . . . . . . . . . . 57

3.4. Syntactic Methods in Another Domain . . . . . . . . . . . . . . . . . . 62

3.4.1. Syntactic Models to Structure Room Scenes . . . . . . . . . . . . . 62

3.4.2. An Experimental Implementation . . . . . . . . . . . . . . . . 64

3.5. Summary . . . . . . . . . . . . . . . . . . 67 
4. Mating Feature Graphs $\quad \mathbf{7 1}$

4.1. Different Syntactic Structures . . . . . . . . . . . . . . . . . . . 72

4.2. Graphs as the Semantics of Syntactically Derived Plans . . . . . . . . . . 75

4.2.1. Relational Modeling . . . . . . . . . . . . . . . . . 75

4.2.2. Labeled Graphs . . . . . . . . . . . . . . . . . . . . . 77

4.2.3. Computing Mating Feature Graphs . . . . . . . . . . . . . . . 81

4.2.4. Intermediate Summary . . . . . . . . . . . . . . . . . . 87

4.3. Matching Mating Feature Graphs . . . . . . . . . . . . . . . . 88

4.3.1. Assembly Recognition . . . . . . . . . . . . . . . . . . . 90

4.3.2. Assembly Reconstruction . . . . . . . . . . . . . . . . . . 95

4.4. Summary . . . . . . . . . . . . . . . . . . . 100

4.5. Intermediate Conclusion . . . . . . . . . . . . . . . . . . 102

5. Temporal Context 105

5.1. Concept of a Visual Memory . . . . . . . . . . . . . . . . . 106

5.2. An Experimental Implementation . . . . . . . . . . . . . . . . . . . 107

5.2.1. Matching Interest Points . . . . . . . . . . . . . . . . . . . . 109

5.2.2. A Performance Example . . . . . . . . . . . . . . . . . . . . 113

5.3. Summary . . . . . . . . . . . . . . . . . . . . 115

$\begin{array}{ll}\text { 6. Evaluation } & \mathbf{1 1 7}\end{array}$

6.1. Prototypical Detection Results . . . . . . . . . . . . . . . 118

6.2. Assessing the Difficulty of a Task . . . . . . . . . . . . . . . . 119

6.3. Results . . . . . . . . . . . . . . . . . . . . . . 124

6.4. Summary . . . . . . . . . . . . . . . . . 126

$\begin{array}{lr}\text { 7. Conclusion } & 129\end{array}$

A. Further Notes on Mechanical Assemblies 133

A.1. Nonuniqueness of Context Free Assembly Structures . . . . . . . . . . . . 133

A.2. Mating Features Resulting from Assembly . . . . . . . . . . . . 133

$\begin{array}{ll}\text { B. Details on Assembly Detection from Parsing } & 139\end{array}$

B.1. An Example of Conjoining Assembly Trees . . . . . . . . . . . . . . . . . 139

B.2. An Example of Instantiating Assembly Concepts . . . . . . . . . . . . . . 141

$\begin{array}{ll}\text { C. Formal Proofs } & \mathbf{1 4 5}\end{array}$

C.1. Ernest and Context Free Grammars . . . . . . . . . . . . . . . . . 145

C.2. Partial Orders . . . . . . . . . . . . . . . . . . . . . 148

D. Complete Definition of the Semantics Function $\mathcal{S} \quad 151$ 
Bibliography

Index 


\section{Introduction}

Imagine two children playing together. Imagine they are playing with a wooden construction-kit and pretend to be "instructor" and "constructor". One is the instructor who plans and tells what to do. The other child plays the constructor and accomplishes his playfellow's instructions. At the beginning of their play they agreed to construct a locomotive. After they have played for short a while, this is what they see in front of them:

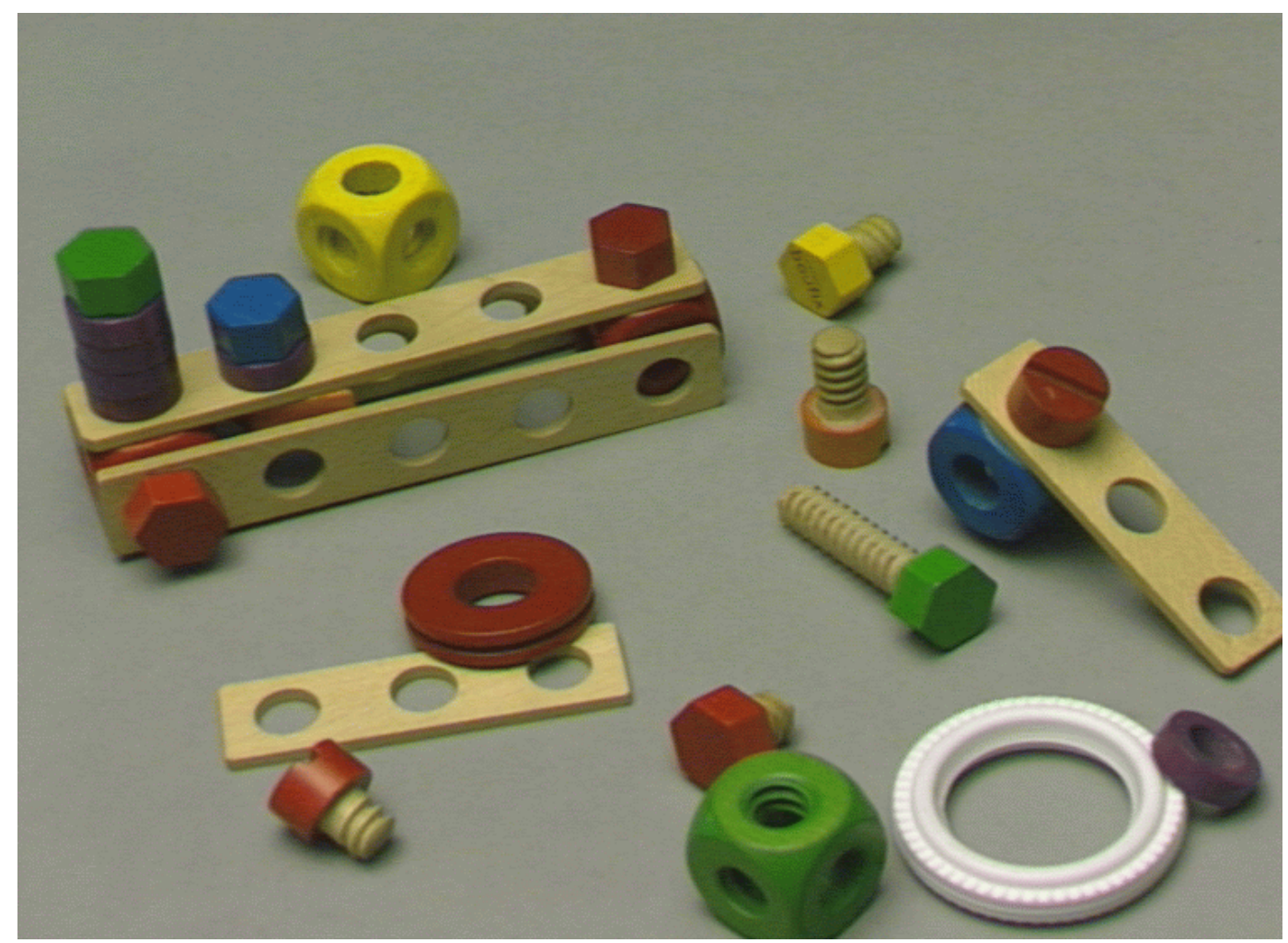

Figure 1.1.: A scene of assemblies and elementary parts of the baufix ${ }^{\circledR}$ construction-kit.

In this situation the instructor requests: "Take one of the little bolts and attach it to the rear of the steam engine!' 'In order to react appropriately the constructor has to conclude what he was told to do. First, he has to relate the verbal 
instruction to the scene he observes, then he must think about the instructor's intention and finally he must derive and carry out a suitable sequence of actions. As he was asked to manipulate one of the little bolts and the steam engine, he has to look for a little bolt and a steam engine. At the moment the child can see several objects and since some of them are bolts, he has to search those for a little one and pick it up; altogether an easy task. Searching for the steam engine, however, is not that easy and requires more thought and abstraction. It is more difficult because the scene does not contain a real steam engine. But there are two complex objects which the children have assembled earlier and maybe the instructor did refer to one of these.

Identifying the intended complex object would be easy if the instructor had pointed to it. Likewise the constructor would know immediately which assembly to manipulate if one of them had been introduced as steam engine before. But let us assume that both is not the case. Thus the constructor has to examine both assemblies and must decide which might depict a steam engine. As we would expect from Fig. 1.1, he chooses the large assembly in the back. Its shape resembles a longish barrel and there are structures sticking out of it like chimneys of a locomotive. However, recognizing the steam engine is not enough to complete the task, the constructor's part is not yet done. Next he must take the engine and attach the little bolt to it. To this end the child should know what part of the assembly is referred as the rear of the steam engine and he has to decide how to grasp the assembly so that the required construction can be accomplished. As the reference to the rear of the steam engine does not precisely specify how to connect the bolt and the assembly, the constructor has two possibilities. He might ask the instructor for more details concerning the attachment or he might follow his intuition and assemble the objects according to his preferences. If these do not coincide with the instructor's intention, the instructor will surely intervene and explain her idea of the attachment more precisely. Thus, through communication and cooperation the children will coordinate their perceptions. They can adjust their interpretations of the scene and the instructions and will finally succeed in constructing the locomotive.

\subsection{The SFB 360}

Amazingly, even for little children all the tasks exemplified above do not pose a real problem. In fact, the parts of the baufix ${ }^{\circledR}$ construction-kit shown in Fig. 1.1 are toys to be played with by children from the age of three. At that age already, children effortlessly perform sophisticated cognition tasks. They are able to plan and carry out the construction of toy objects. They can talk about toy assemblies and recognize them as models of real world objects. These astounding capabilities of the human brain even at early stages of its growth become even more impressive if one tries to implement them on a machine; decades of intensive research have revealed how difficult this is.

Nevertheless, scientific efforts towards intelligent systems with complex cognitive ca- 
pabilities have increased significantly throughout the last years. The main motivation is to enable unconstrained, natural, and human-like man-machine communication. More and more projects aim to construct smart and cognitively skilled machines that should cooperate with or assist human users. Although commercial solutions are not yet available, scientists already predict many applications for communicating machines. Among the most mentioned are service robots, tele-operated machines in hazardous environments, or interactive toys. In fact, game playing scenarios like in our example are a popular setting for research on intelligent man-machine interaction. They are simple but allow to study numerous phenomena of human cognition, communication, and behavior. Examples of work towards playing machines are the PLAYBOT project by Tsotsos et al.[128], the CELL system by Roy [110], and the Collaborative Research Center 360 (SFB 360) at Bielefeld University (cf. [106]) where this thesis emerges from.

Within the SFB 360 computer scientists, linguists, and psychologists are trying to understand the mechanisms of interpersonal communication in order to develop a situated artificial communicator. Interested in advanced human-computer interaction they construct a machine which is situated in a certain environment and processes visual and acoustic input to understand a human's verbal and gestural instructions. Not only should the machine understand its human vis-a-vis but it also should react appropriately and accomplish what it was told to do. The scenario for this research was chosen to be the cooperative construction of toy models of, for instance, airplanes, trucks, or locomotives using parts from the baufix ${ }^{\circledR}$ construction-kit.

Developing a machine like that is definitely a demanding task: it requires the integration of automatic image and natural speech understanding, it requires to implement dialogue strategies and it requires the capability to manipulate physical objects. And as the artificial communicator should perform assembly tasks, it furthermore requires that the machine can deal with mechanical assemblies. Apparently, the latter requirement is less specific than the other ones. It is not immediately clear if it raises any problems and of what nature these might be. Therefore, we shall have a closer look at this issue and will discuss what intelligent man-machine interaction for cooperative assembly is all about.

\subsection{Aspects of Assemblies in Cooperative Construction}

First efforts in research on intelligent assembly robots date back to the early seventies of the last century. In 1975 Ambler et al. [3] reported on an assembly system equipped with two cameras, a moving table, and a manipulator that was able to assemble up to 10 simple toy objects with pegs and holes as shown in Fig 1.2. The system was designed to interact with a human and had three phases of operation. In the first phase an instructor successively placed different objects under an overhead camera, each in several orientations. The system took pictures of the objects and generated relational 

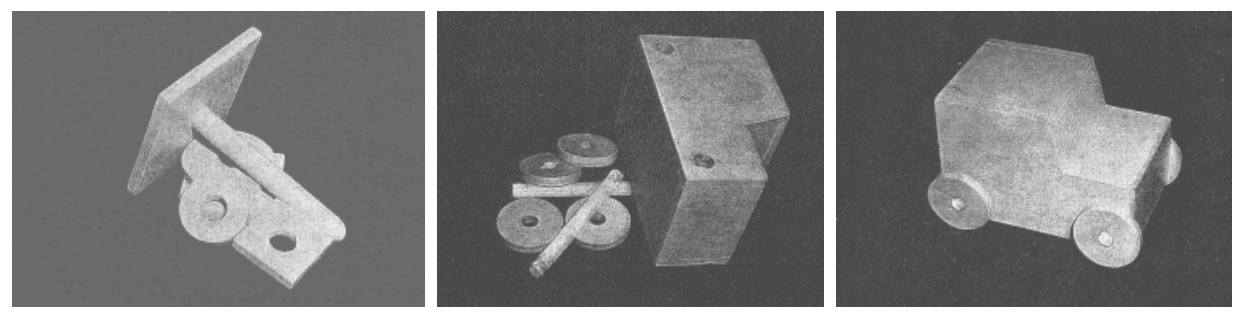

Figure 1.2.: Examples of objects Ambler et al. [3] were dealing with in 1975. Pictures reprinted from [3], (c)1975, with permission from Elsevier Science.

descriptions which were labeled by the user. The descriptions were based on attributes of regions which had been extracted from image data. To facilitate the necessary computations Ambler and his coworkers considered bright objects on a dark background. The model acquisition phase was followed by a teach-in session where the objects were put into standard positions and the machine was programmed to take and to assemble them. In the operating phase a couple of objects were arranged in heaps on the table (see Fig. 1.2). The system's side camera was used to detect those heaps; for object identification detected heaps were moved under the overhead camera and the system employed preprogrammed strategies to rearrange the heap in order to recognize the resulting entities. Recognized objects were taken and put into their standard position. After all parts of a heap were separated and put into standard positions, the system started to run the preprogrammed assembly sequence.

An astounding and important characteristic of this system was that it performed all this without any knowledge of assemblies. Of course, it was equipped with implicit assembly knowledge, otherwise it could not have constructed complex objects. But any knowledge concerning assemblies was encoded in the standard positions of parts and the preprogrammed sequences to manipulate them. As soon as all the parts were located in their feeding positions, the system just repeated the predetermined manipulator motions and did not behave intelligently anymore ${ }^{1}$. Of course, realizing such a system was a great achievement in the 1970s. But from a present-day perspective its flexibility in assembly was rather marginal.

The main reason for the machine's low adaptability rests in lack of interaction between the system and its instructor. Human-computer cooperation was limited to the presentation and naming of objects in several positions and to classical teach-in programming of manipulator sequences. During the assembly phase the instructor and the system did not interact. Consequently, there was no need for the system to have knowledge of assemblies. As the authors admit, this resulted in a loss of flexibility: the system

\footnotetext{
${ }^{1}$ It had a force sensor to control grasping and therefore was able to cope with slightly misplaced objects, thus there was little intelligence left in the assembly phase.
} 
was nearly unable to cope with errors. If, for instance, a part required in an assembly sequence was missing or fell down after grasping, the system could not finish its task.

In the SFB 360 scenario cooperation between human and machine is closer and the role of assemblies is more important than in the work of Ambler and his colleagues. As our introductory example of the playing children indicates, cooperative assembly is heavily centered on assembled objects. Instructor and constructor refer to them, they name them and they discuss their appearance or structure. Therefore assemblies as such cannot be neglected when designing a system for cooperative assembly.

Even if cooperative assembly only has to deal with simple toy objects, the issues that must be regarded can become quite complex. Yet in the simple scenario of the SFB 360 assemblies are subject of many questions and give rise to diverse problems. Research on artificial communicators for cooperative construction must at least consider their following aspects:

- Planning: to the instructor assemblies appear as a problem of planning. She has to determine sequences of assembly tasks and, if necessary, must modify originally planned steps.

- Structure and Process: the constructor experiences assemblies as evolving complex structures. They are intermediate steps of a construction process resulting from manipulations requested by the instructor.

- Learning: assemblies also challenge the constructor's skills in adaption and learning. Using experience or intuition to anticipate what the instructor has planned generally speeds up construction.

- Semantics: not every assembly depicts a real world object, but during a construction process assemblies may become instances of real life entities.

- Pragmatics: assemblies also have pragmatic aspects. Introducing names for complex objects usually establishes intrinsic orientations (like in the case of the rear of the steam engine). This generally simplifies further instructions as the partners can use intrinsic orientations to coordinate their perception.

- Recognition: realizing an artificial communicator obviously requires to solve problems of speech and image understanding. Assemblies or references to assemblies have to be recognized in visual and acoustic data.

- Simulation: human-computer interaction can be studied in virtual worlds as well. Thus it is desirable to have a virtual reconstruction of physical properties of assemblies in order to simulate manufacturing.

- Motion Planning: for an intelligent robot assemblies also pose problems of trajectory planning and grasp control for object manipulation. 
Treating any of these aspects by means of a machine necessarily requires to provide data or knowledge of assembled objects. Looking for adequate representations, however, is not a trivial problem and a general framework for assembly modeling has not yet been introduced. Especially the integrated treatment of different facets of flexible and cooperative assembly remains an interesting topic of research as only few projects about the world deal with similar problems. However, some of the aspects listed above do not exclusively arise in developing an artificial communicator to support assembly but are familiar problems in research on robotics and automation.

In 1980 Nevins and Whitney [91] for the first time systematically explored issues of importance in robotic assembly. Their interest in assembly did not come from an artificial intelligence perspective but was driven by economical considerations. Nevertheless, they identified problems like simulation, automatic planning and inspection to be of primary concern for cost reduction in manufacturing. Numerous approaches to these problems have been published ever since, many of them focused on issues of representation. By now computer aided modeling and planning for intelligent mechanical assembly have become established fields of research. Most of the techniques developed so far are designed to be used in industrial CAD or CAM/CIM systems. Even if these methods do not primarily aim at intelligent human-computer interaction, they provide important hints for the scenario of the SFB 360 and relevant contributions will be reviewed in Chapter 2 .

\subsection{Assemblies and Computer Vision}

This thesis addresses the problem of assembly recognition from computer vision. In their early work on general issues in robotic assembly Nevins and Whitney [91] already mentioned the potential of vision in assembly and manufacturing. According to their study the main benefit offered by this technique lies in the field of inspection and error detection. A more recent article by Noble [93] underlines that computer vision has indeed become a customary tool in industrial manufacturing. However, the use of computer vision to deal with problems of higher complexity than inspection or error detection is still in its infancy. Challenging vision tasks in robotic assembly like, for instance, process understanding or learning from demonstration in an arbitrary domain are topics of research but have not yet been applied in industry.

The absence of industrial solutions indicates that issues in visual assembly recognition are complicated and complex. How difficult they are can be also seen from literature: in spite of undeniable progress in vision and robotics since the pioneering work by Ambler et al.[3], research on assembly observation is still restricted to rather simple objects. The examples in Fig. 1.3 illustrate that even recent contributions only consider simply shaped and textured objects. As this drastically facilitates object modeling for recognition, it seems that knowledge representation still is a crucial issue in vision for assembly.

Every pattern recognition task requires a suitable representation of the instances 


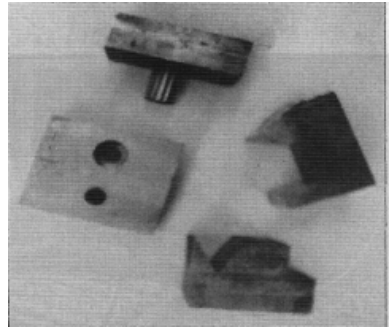

(a) Tung and Kak [129]

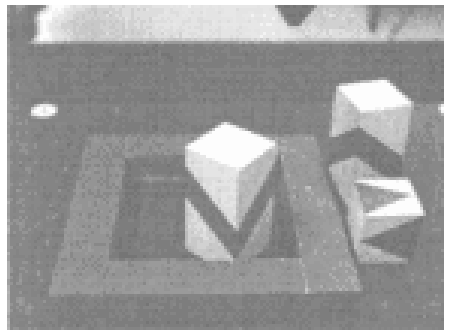

(b) Lloyd et al. [78]
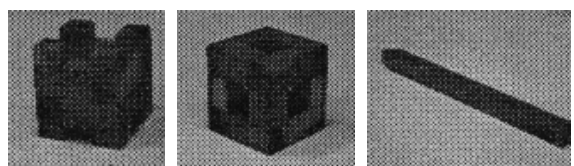

(c) Ikeuchi et al. [61, 62]

Figure 1.3.: Examples of elementary objects considered in recent contributions to visual assembly recognition and process understanding. (Pictures (c)1996, 1999, and 1992 IEEE).

that should be recognized. In computer vision, recognition usually is based on features extracted from the image signal. Possible features might be geometric entities like points, lines, homogeneous regions, sets of points, or patches of texture; or they might be of more abstract nature like color histograms, Fourier transforms, or indices derived from simpler (geometric) features. Features can represent global or local properties of objects. Global features characterize objects as a whole. They are well suited to process images that were recorded under standardized conditions and show few isolated objects. When dealing with partially occluded objects or cluttered images global features mostly are improper to attack the task. Local features capture local properties of objects which should be invariant under changes of perspective or illumination. Often relations among sets of local features are used to generate specific representations. The problem about local features is to find appropriate invariants which are sufficiently characteristic to enable reliable object recognition.

The use of global features is usually associated with schemes of implicit knowledge representation. Based on statistical inference or optimization processes which usually require large sets of training samples, approaches like statistical classifiers, neural network, Markov random fields, or Bayesian networks provide prototypical object models. Local features, on the other hand, are often used in explicit knowledge representation. Recognition systems based on predicate logic, grammars, or semantic networks encode relations among local features that usually have to be modeled by a knowledge engineer.

As this thesis is embedded in the SFB 360, the visual recognition of the bolt-nut type assemblies from the baufix ${ }^{\circledR}$ scenario is our primary concern. This of course requires a suitable method of knowledge representation. Since the artificial communicator should neither operate in a standardized environment nor be restricted to the construction of a certain type of toy models, knowledge representation must be flexible and able to deal with as much feasible assemblies as possible. Therefore, implicit modeling seems inappropriate. Consider for example the assembly shown in Fig. 1.4 which depicts the 


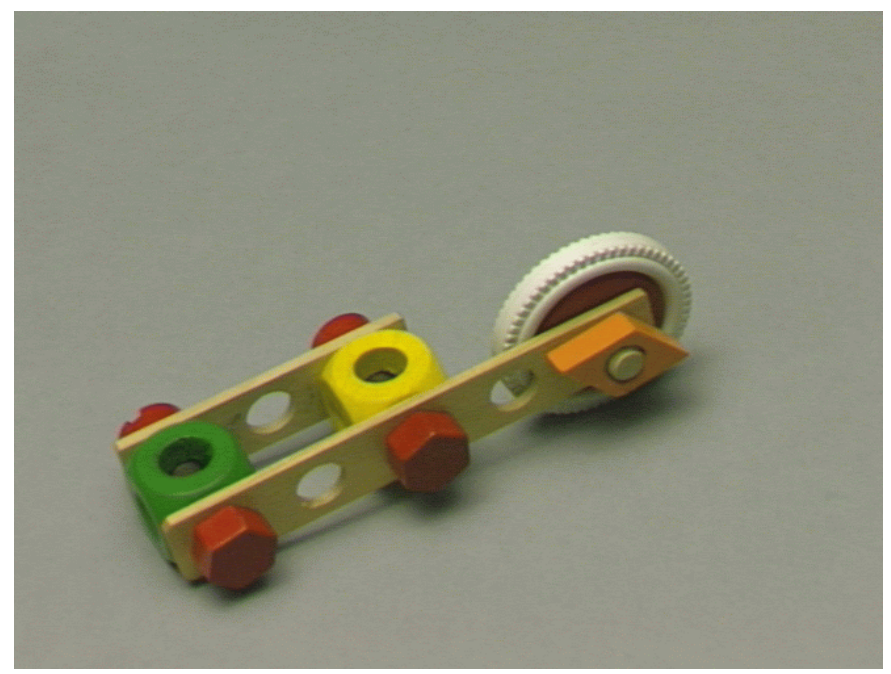

Figure 1.4.: A baufix ${ }^{\circledR}$ assembly depicting the wheel fork of a scooter.

wheel fork of a scooter. Its appearance in an image heavily depends on the perspective thus many different images of this assembly would be necessary only to gain reliable wheel fork recognition. Hence, training a general purpose assembly recognition system based on implicit knowledge representation would mean enormous effort. For the same reason, any collection of any kind of individual models also seems improper. Since flexible recognition would require huge amounts of individual assembly models, other approaches to knowledge representation are required. In the following we will argue that flexible and reliable recognition can be acquired by means of generic models combined with learning mechanisms.

\subsection{Organization of this Thesis}

Mechanically, all baufix ${ }^{\circledR}$ assemblies are composed according to a small set of rules. Within a feasible assembly every part plays a certain role which is constrained by these rules and characterizes its relations to the other parts. In Chapter 3, we will introduce a general model of the class of all baufix ${ }^{\circledR}$ assemblies that subsumes the construction rules of and takes into account the functional aspects of parts of an assembly. This model is recursive and corresponds to a simple grammar and thus allows to derive a syntactic structure for every assembly in our scenario. It was implemented in the semantic network language ERNEST (cf. $[71,113]$ ) and is used to detect assembly structures in images of baufix ${ }^{\circledR}$ objects.

As Chapter 3 will show, syntactic descriptions of assemblies are closely related to assembly sequence plans and we will introduce a method to generate plans from vision. 
Due to this close relation, the syntactic model also allows to approach the problem of action monitoring.

A frequent problem in assembly recognition arises from perspective occlusions. In Fig. 1.4, for example, we can see that the wheel is attached to the fork even though the bolt responsible for this attachment is not visible. Though it is occluded by the wheel we can infer the bolt's presence from the visual context. Chapter 3 discusses how contextual knowledge provided by the syntactic model allows to hypothesize the presence of elementary objects even if they are just partially visible or totally occluded. Integrating this technique into a feedback loop with an elementary object recognition process improves the performance of the artificial communicator's recognition modules.

Considerations about the function of the parts (or variables) of the syntactic model revealed that recursive descriptions may be found for any type of structured set of objects. Another interesting aspect about recursive models of assemblies is that they are tightly related to discourse theory. These theoretical issues are discussed in Chapter 3 as well and we introduce a framework to facilitate the search for recursive models. Based on this framework the application of the syntactic approach to another domain is presented.

Syntactic methods are a long-established in pattern recognition because they are well suited to detect structures of a certain class in various kinds of data (cf. e.g. [39, 97]). However, they are not that well suited to individual assembly recognition because individual assemblies usually entail several grammatical derivations. As we will explain in Chapter 4, most mechanical assemblies will even come along with many different syntactic structures. But even if two descriptions of an assembly are syntactically different, on a semantical level they are equivalent for they denote the same object. Thus, the recognition of individual assemblies should be based on the semantics of syntactic descriptions. Chapter 4 introduces a graph representation which captures relevant relations among parts of an assembly. It constitutes an interpretation of syntactically derived assembly structures and we will present a denotational semantics function to calculate graphs from syntactic structures. This, in fact, realizes a learning mechanism for explicit object models, i.e. our system can learn individual representations from image analysis.

Based on graphs object recognition becomes a problem of graph matching. In Chapter 4 we will describe the use of a matching tool developed at the University of Berne. It is able to find exact and error tolerant matches between graphs which allows to treat a whole class of problems: Chapter 4 presents the recognition of assemblies, the recognition of similar assemblies, and the recognition of subassemblies.

Furthermore, matching assembly graphs also enables 3D reconstruction. 3D object recognition from image data is usually based on stereo vision and poses particular problems. In his book on three-dimensional computer vision, Faugeras notes [34, page 165]:

The main difficulty is to come up with good answers for the following so called correspondence problem: Given a token in image 1, what is the corresponding token in image 2 ? 
Usual approaches to this problem apply techniques from low level vision. They try to establish correspondences by searching for features of the one image similar to those of the other image (cf. e.g. [117]). Chapter 4 will present a high-level vision approach. Given stereo images of assemblies correspondences can be found by structural matching of symbolic information.

Generally, syntactic and structural knowledge representation for assembly recognition is flexible and economical for it can deal with a wide variety of objects without representing numerous individuals. It also yields reliable results and thus it is reasonable to be used in the scenario of the SFB 360. However, syntactic methods can be expansive in terms of computation time. Syntactical pattern analysis often has to check many alternatives to derive the best possible explanation of an input pattern. Generally, it is easy to examine patterns of few constituting parts. But if the number of primitives increases, syntactic analysis will suffer from combinatorial explosions and thus may last a while. However, in the SFB 360 we are dealing with construction processes and therefore can propagate object information through time which, as Kummert et al. [73] proved in earlier work, can considerably speed up object recognition. This, of course, requires a mechanism to store results from previous object recognition.

Chapter 5 will describe a system to store and associate different types of information from visual assembly process monitoring. This memory has a multidimensional structure since it registers data from different levels of image processing. If new information is drawn from image processing, it is stored and related to earlier derived facts. By this the memory dynamically learns prototypical features for assembly recognition. It is scalable since new dimensions can easily be integrated into the architecture and it is modular because methods to compare data of a certain dimension can be exchanged. From a system architecture point of view this memory represents a hybrid architecture coupling methods based on global and local features to represent assemblies.

A performance analysis of our syntactic approach to assembly detection as well as an analysis of the hybrid system that exploits process information for assembly recognition will be presented in chapter 6 . As we will explain, evaluating the results yielded by an assembly recognition method must take into account how difficult the recognition task was. It will be discussed how this difficulty could be measured and a solution to the problem based on linguistic variables will be introduced. Chapter 7 will summarize and conclude the topics of this thesis. 


\section{Assembly Modeling and Monitoring}

Mechanical assemblies are almost omnipresent in our everyday life. Man-made objects of utility like tools, toys, furniture, vehicles, electronic devices, and all sorts of machines are usually composed of simpler parts. And as most assembled artifacts are mass products machined in highly automated plants, manipulating assemblies by means of machines seems easy. But it is not. Even though automation has become an off-the-shelf technology, automatic manufacturing is by no means trivial. In fact, it requires careful shop floor design as well as process planning or scheduling and the installation of monitoring devices for failure detection.

These are tasks usually accomplished by human experts. Production engineers plan assembly sequences, mechanisms of part feeding and manipulation, and ways of quality control. They must thereby consider physical properties of the incorporated parts as well as of the final product and as industrial products are exposed to economic competition, their designs have to be economically reasonable. Hence, yet before they physically exist, mechanical assemblies pose difficult optimization problems which are subject to constraints of temporal, spatial, physical and economical nature.

Trying to solve problems like these by means of intelligent machines is obviously challenging. Nevertheless, encouraged by progress in artificial intelligence and robotics (and of course driven by economic motivations) an increasing number of researchers addresses automatic planning and intelligent manipulation of composite objects. Moreover, interest in man-machine interaction for assembly and automatic perception and recognition of assembled objects have gained attention nowadays. Such efforts are, for instance, made at Bielefeld University where the Collaborative Research Center 360 explores advanced man-machine communication in an assembly scenario. The setting was introduced in the previous chapter where we also identified and discussed different aspects of assemblies arising in its scope. We saw that there are problems that go beyond sequence or trajectory planning for they require to face demanding cognition tasks. Issues like process understanding, complex object recognition, and semantics and pragmatics of assemblies throughout a dialog must be dealt with. Thus, in intelligent cooperative construction assemblies necessitate the integration of various techniques and methods known from artificial intelligence that usually are studied separate from one another.

Industrially motivated assembly research as well as basic research in cooperative assembly do, of course, require suitable schemes of knowledge representation. Without an abstract representation of assembly knowledge none of the above problems can be 
solved algorithmically and therefore can hardly be treated by intelligent machines. Unfortunately, due to the variety of aspects and difficulties there is yet no general framework to represent assemblies. But literature on assembly modeling is rich and many methods focused on certain special questions can be found. Thus, owing to its importance within the SFB 360, we will review relevant and representative approaches in the following. We will also review work on mathematical complexity in assembly modeling because it yields insight of practical importance. And since it is the main objective of this thesis, we will examine to what extend known methods can be applied to visual process monitoring and assembly recognition. But first of all we need a precise definition of what mechanical assemblies are.

\subsection{Definition of Mechanical Assemblies}

Many of the contributions introduced in the following do not provide a formal definition of mechanical assemblies. Requicha and Wahlen [105], in contrast, thoroughly examine this topic and present several mathematical definitions of assemblies composed of rigid parts. To them an assembly instance $\alpha$ consisting of $n$ solid objects is a pair $\alpha=(\sigma, \tau)$ where $\sigma=\left(S_{1}, S_{2}, \ldots, S_{n}\right)$ is a collection of solids and $\tau=\left(T_{1}, T_{2}, \ldots, T_{n}\right)$ is a tuple of transformations. The authors carefully point out that $\sigma$ is a point of the solid configuration space $C_{s}=M_{s} \times M_{s} \times \ldots \times M_{s}$ which is the product of $n$ copies of $M_{s}$, the modeling space for solids. Likewise, the transformation configuration space $C_{t}$ is the direct product of $n$ copies of $M_{t}$, the modeling space for transformations. As rigid objects in $\mathbb{R}^{3}$ have three positional and three orientational degrees of freedom, $M_{t}$ is a six-dimensional space. The structure of $M_{s}$ is more complex for it is the space of $r$-sets in $\mathbb{R}^{3}$ where $r$-sets are compact, regular, semi-algebraic subsets of $\mathbb{R}^{3}$ used to model individual solids (cf. [104]).

To cope with phenomena due to pose uncertainty of parts, Requicha and Wahlen define a nominal assembly to be a set of assembly instances:

$$
A(\sigma, \Theta)=\left\{\alpha(\sigma, \tau) \mid \tau \in \Theta \subseteq C_{t}\right\} .
$$

As it cannot be guaranteed that parts are manufactured with perfect shape, they introduce variational assemblies to take part variability into account:

$$
A=\bigcup_{\sigma \in \Sigma} A(\sigma, \Theta)
$$

where $\Sigma$ is a subset of $C_{s}$.

Finally, Requicha and Wahlen underline that not all $\alpha=(\sigma, \tau)$ correspond to feasible assemblies. The set of realizable assemblies is restricted by the conditions of noninterference and path-existence which guarantee that no two objects share the same region of space and that for every part there is a collision free path to be integrated into the assembly. 
A less formal, more intuitive but widely accepted definition of mechanical assemblies is given by Rabemanantsoa and Pierre $[100]^{1}$ :

A mechanical assembly is a set of interconnected parts representing a stable unit in which each part is a solid object. Surface contacts between parts reduce the degree of freedom for relative motion. Attachments by means of screw, glue or pressure act on surface contacts and eliminate all degrees of freedom for relative motion.

The construction of such an assembly can be described as a sequence of assembly tasks in each of which subassemblies are joined to form larger units. Subassemblies are defined to be subsets of parts which either contain a single element or a number of rigidly connected elements. Thus, as Jones and Wilson [63] point out, a subassembly can be treated as if it were a part. According to Wolter [135], sets of non-rigidly connected parts which do not constitute stable units are called partial assemblies. For the remainder of this thesis we will keep this terminology.

\subsection{Geometrical Assembly Modeling}

Most published methods to represent assembled objects belong into one of the two following categories: on the one hand, geometric models describe the spatial position and orientation of individual objects and thus allow to register physical contacts among the parts of an assembly. On the other hand, there are more abstract descriptions of assemblies reflecting topological, semantical or hierarchical structures of mechanical artifacts. Most contributions outlined in the following integrate both approaches but stress either the one or the other.

Geometric models are unique representations of physical objects. Popular methods to implement such representations are Boundary Representations (BRep) and Constructive Solid Geometry (CSG). BRep methods describe objects by means of graphs whose vertices contain parameterized descriptions of object surfaces while CSG approaches model physical objects as a union of certain predefined volumes (cf. [104]).

Examples for the use of BRep methods in assembly modeling can be found in the work of De Floriani and her coworkers [16, 29]. In addition to boundary models they developed a graph representation to record surface contacts between individual objects so that these easily can be unified to form more complex units. Anantha, Kramer and Crawford [5] present an approach where they enhance boundary models by domain specific knowledge of possible contact relations to facilitate the automatic analysis of a part's mechanical degrees of freedom. A similar method to simulate construction processes is presented by Thomas and Nissanke [125, 127]. They introduce a graph representation

\footnotetext{
${ }^{1}$ In fact, this definition is adopted from an earlier one by Homem de Mello and Sanderson [54].
} 
of the geometrical properties of objects and define an algebra of graph merging operations to virtually solve construction tasks. A contribution by Rabemanantsoa and Pierre [100] is again based on a degree of freedom analysis of parts comprised in an assembly. Using CSG representations of individual objects and information about possible interconnections calculated from the models they derive assembly sequences for composite objects.

In the works of van Holland, Bronsvoort, and Jansen [130] and Caracciolo et. al. [23] most attention is paid to contact relations between mating features of assembled objects. In fact, the idea to model geometric properties of mating features like bolt and hole and to examine possible relations among them can already be found in an early contribution by Ambler and Popplestone [4]. Beyond mere geometric properties Laperrière and ElMaraghy [74] and Röhrdanz, Mosemann, and Wahl [109] also simulate effects of gravity in order to test the physical stability of object connections. Besides the authors cited so far, Fuh, Chang, and Melkanoff [41], Kruth, Van Zeir, and Detand [70] as well as Linn and Liu [76] also examine geometric aspects of mating features. They also deal with robotic assembly but concentrate on automatic mating feature identification from geometrical models.

Eng et al. [32] use kinematic-pair-liaison diagrams which characterize the relations among the mating features of two parts. Transforming these diagrams into matrices which can easily be combined allows to represent the contact state of an object with respect to other ones. Mäntylä and his coworkers [48, 103] realize a multigraph representation of assemblies and besides geometric contact information include the function of parts so that man-machine interaction in computer-aided workpiece design becomes more intuitive. Dealing with assembly sequence planning Hong and Cho [56] define hypothetic costs for the connection of triples of parts. The costs are derived from geometric details of object contacts and are used to define energy functions of a Hopfield net. After cycling through an energy minimization process, the net reaches a stable state which corresponds to an optimal sequence.

Ruiz and Ferreira [111] consider assembly modeling from a theoretical point of view. They propose a representation in canonic coordinates and show that their approach is advantageous in terms of computational efforts. In another rather theoretical contribution Arbab [6] develops a set theoretic formalism to provide a mathematically sound description of the union of several CSG object models.

\subsection{Structural Assembly Modeling}

Structural methods of assembly modeling are frequently found in contributions concerned with assembly sequence planning. They rather abstract from detailed part geometry and model mating relations among objects on higher levels of conception. Instead of their spatial position interconnected parts are, for instance, characterized by the forces 


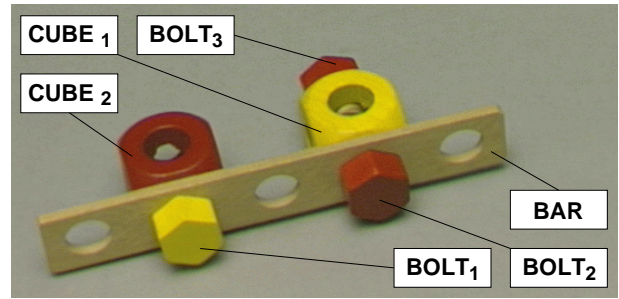

(a)

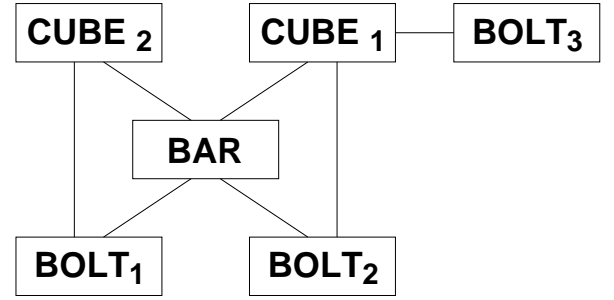

(b)

Figure 2.1.: Example of a baufix ${ }^{\circledR}$ assembly and the corresponding liaison graph representing contact relations among the parts.

necessary to loosen the connection. On the other hand, assemblies and subassemblies are often seen as complex units of semantic or functional significance like, for example, the tailplane fin of an airplane.

One of the earliest contributions pursuing a structural approach was presented by Chang and Lee [25] in 1988. They used frames to model knowledge of complex objects and their parts. At the same time, Neumann [90] surveyed expert systems in assembly and product design and elaborately discussed how to draw inference from hierarchical models. Chakrabarty and Wolter [24] also introduce a representation of component hierarchies to model assemblies and make use of databases to store plans of subassemblies which can be merged and facilitate modeling of similar assemblies.

Hsu, Fuh and Zhang [58] describe manufacturing processes by means of state transition diagrams where assemblies are considered as in- and output of a construction step. Thus, physical properties of parts play a secondary role while their function is of primary concern. Another method taking into account that assemblies result from manufacturing processes are precedence graphs which, among others, are described by Chen and Henrioud [26]. Precedence graphs display precedence relations among individual assembly tasks and thus allow to identify possible assembly sequences as well as tasks that can be accomplished simultaneously. The work of Chen and Henrioud is based on liaison graphs which they use to derive all minimal precedence graphs of a product. Liaison graphs were introduced by Bourjault [17] who proposed them as a method to model contact relations among parts².

Liaison graphs neglect details of part connections, they simply summarize all physical contacts within an assembly and thus describe its topological structure. An example of this important concept is shown in Fig. 2.1. Each vertex of the graph in Fig. 2.1(b)

\footnotetext{
${ }^{2}$ Remarkably, this contribution (which Bourjault wrote as his thesis) is among the most cited works in sequence planning literature although it is written in French. Other descriptions of this concept can, for instance, be found in further work by Henrioud and Bourjault [51] or in a more recent article by De Fazio, Rhee, and Whitney [28].
} 
represents an object of the assembly shown in Fig. 2.1(a). Objects related by an edge are in physical contact: the object labeled BAR, for instance, has surface contacts with the objects named BOLT 1 and $\mathrm{CUBE}_{2}$. Since $\mathrm{CUBE}_{2}$ is threaded onto BOLT $\mathrm{T}_{1}$, they are related by an edge as well although this type of surface contact is not visible in the image. Note that in the example the edges of the graph are unlabeled; many contributors, however, assign labels to the edges in order to further specify object relations. It is, for instance, quite common to characterize the type of connection. Had we done this in our example, the edge relating $\mathrm{BOLT}_{1}$ and $\mathrm{CUBE}_{2}$ could have been labeled threaded cylindrical hole insertion, the one between BOLT $\mathrm{B}_{1}$ and BAR might have been labeled cylindrical hole insertion and the relation between $\mathrm{CUBE}_{2}$ and $\mathrm{BAR}$ could have been characterized as planar surface contact.

Stadzisz and Henrioud [121] point out that liaison graphs are similar for whole families of products. They introduce graph grammars to generate liaison graphs automatically and use Petri nets to represent generic assembly plans. Liaison graphs whose edges are labeled by indicators of the force necessary to loosen the corresponding contact are applied by Lee and Wang [75]. This yields models obviously closer to the physical world than the mere topological representations provided by classical liaison graphs. Two further closely related methods of assembly modeling are block graphs developed by Qian and Pagello [99] and non directional blocking graphs described by Goldwasser, Latombe, and Motwani [46]. These representations specify which objects of an assembly can not or can be moved with respect to the other objects, respectively. Hence, physically feasible decompositions of assemblies can be calculated without detailed geometric knowledge. Both concepts, however, should be subsumed in the class of liaison graph methods for they basically correspond to liaison graphs with an edge labeling scheme chosen accordingly.

Another popular method to model mechanical assemblies is due to work by Homem de Mello and Sanderson $[53,54,55]$. They propose the use of $A N D / O R$ graphs to summarize all feasible possibilities to construct an assembly.

AND/OR graphs are well known from heuristic problem solving (cf. [98]). They can be regarded as a generalization of ordinary graphs called hypergraphs. Hypergraphs contain hyperlinks which connect a parent node with a set of successors. In problem solving by means of AND/OR graphs, individual elements of a hyperlink which are called AND link correspond to subproblems of a problem represented in the parent node. If there are several hyperlinks emanating from a node, they are called OR links and represent alternative approaches to subdivide the corresponding problem. In heuristic problem solving one is interested in finding solutions of AND/OR graphs, i.e. a subtree with the following properties:

1. It contains the root node.

2. All its leaves are nodes in the original AND/OR graph with no edges leaving them. 


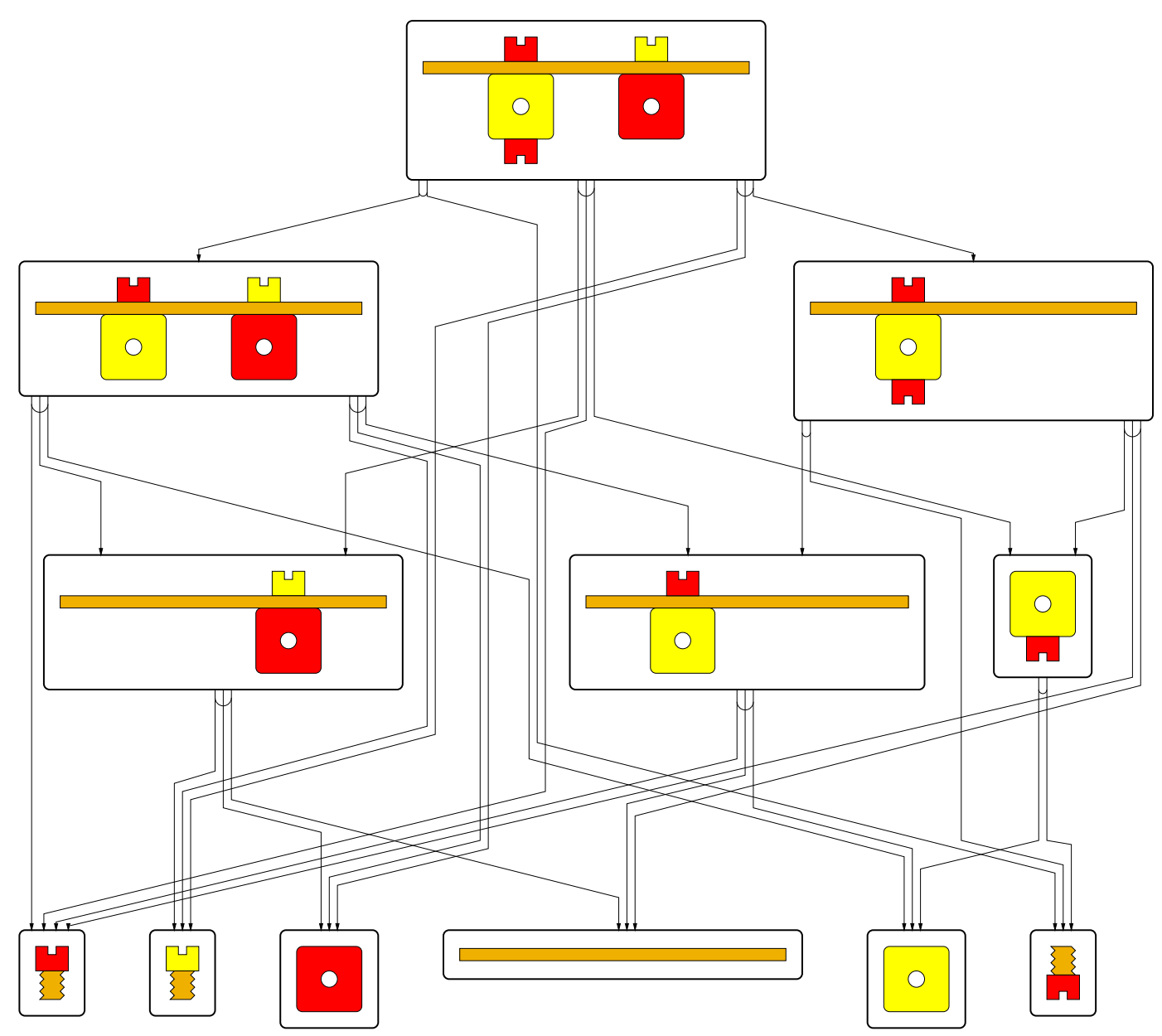

Figure 2.2.: An AND/OR graph summarizing all feasible decompositions of the assembly shown in Fig. 2.1(a). The graph contains five trees, therefore there are five different possibilities to assemble the composite object in the upper most node from the elementary objects in the lower most nodes.

3. If it contains an AND link $a$ of a hyperlink $h$, it must contain all other AND links of $h$.

Homem de Mello and Sanderson propose to identify the nodes of an AND/OR graph with an assembly and its subassemblies and to draw hyperlinks such that every hyperlink corresponds to a geometrically and mechanically feasible decomposition of the assembly in its parent node. If this is done correctly, every solution of the AND/OR graph represents a feasible sequence to disassemble (or vice versa to assemble) the composite object 
in the root node; in assembly sequence planning, the solutions are known as assembly trees.

As the concept of AND/OR graphs is essential for some of the theoretical consideration in the next chapter, let us have a look at an example. Figure 2.2 depicts the AND/OR graph describing the baufix ${ }^{\circledR}$ assembly in Fig. 2.1(a). Each node in the graph contains a sketch of the (sub)assembly it represents. Hyperlinks are illustrated by bundles of edges connected by an arch. If there are several hyperlinks emanating from a node, there are several possibilities to loose a rigid connection, i.e. a connection between a bolt and a nut, of the assembly in that node. Hence, for the assembly in the upper most node, for instance, there are three possibilities to perform a disassembly task. From the fact that the graph contains five assembly trees we learn that given the objects in the lowest row of nodes there are five different sequences to assemble the structure in the upper most node.

AND/OR graph approaches can be found in many publications on assembly modeling, because they provide a comprehensive but compact way to represent part-of structures and, at the same time, encode temporal process knowledge. However, Suzuki et al. [123] point out that it is difficult to handle AND/OR graphs algebraically. Therefore, they map them onto Petri nets which they use to analyze construction processes, an idea also pursued by Thomas, Nissanke and Baker [126]. Finally, for the same purpose Seow and Devanathan [118] apply a framework of temporal logic. They derive logical equations to discuss how mechanical assembly evolve over time.

\subsection{Assembly Modeling within the SFB 360}

In the introduction we described several non trivial problems of importance in the scenario of the SFB 360. We saw that, in a sense, the objects that are being constructed condition the interaction in cooperative assembly: geometrical, temporal, or structural properties of assemblies affect how the cooperating partners express their intentions. Thus, inherent in intelligent interaction for assembly, there are phenomena caused by the assemblies themselves.

A perfect example of such phenomena are depictional metonymies. Metonymies are figures of speech in which the name of one thing is used for the name of another associated with or related to it (e.g. land that belongs to the crown). The notion of depictional metonymies was introduced by Rieser and Meyer-Fujara [107] in order to classify metonymies where things are described by entities that they depict. Conducting linguistic research within the SFB 360, Rieser and Meyer-Fujara observed that this type of metonymy frequently appears in a corpus of exemplary dialogues which were recorded in experiments where subjects had to assemble a toy airplane using the baufix ${ }^{\circledR}$ construction-kit. Shortly after the beginning of most experiments, subjects 
started to talk about wings or propellers that should be attached to the fuselage although there were but models of wings, propellers and fuselages.

Concerned with the semantics and pragmatics of depictional metonymies Rieser and Meyer-Fujara [14, 108] develop syntactic models of some of the (sub)assemblies that were constructed in the experiments. They use General Phrase Structure Grammars to provide specific models for individual assemblies which reflect the sequence of construction the instructor specified in the respective experiment. For the interpretation of their syntactic models Rieser and Meyer-Fujara present a semantics function that maps syntactic structures on metonymies, i.e. pairs of instances $\left(I, I_{\text {depict }}\right)$ where the first component represents a baufix ${ }^{\circledR}$ assembly and the second one describes a concept depicted by the assembly. Finally, the pragmatics of metonymies are characterized by a couple of constraints that were extracted from the experimental data. Putting it all together this constitutes a theoretical framework to study the conditions for and the evolution of the use of metonymies throughout a dialogue. And as the authors demonstrate by means of further examples, their framework is not restricted to the world of baufix ${ }^{\circledR}$ assemblies but can be adopted to other domains.

Further assembly research within the SFB 360 focuses on the dynamics of assembly semantics. Wachsmuth, Jung, and Hoffhenke [52, 64, 132] study changes in the semantics or roles of assemblies during cooperative construction. Since such effects can be found frequently in the dialogue corpus, they try to find out the preconditions for them to happen and how intelligent machines could deal with such phenomena. They introduce an operational knowledge representation formalism called COAR (Concepts for Objects, Assemblies and Roles) to formally reconstruct effects of dynamic conceptualization in sequences of assembly tasks. Assembly processes are simulated by means of a virtual reality tool that contains geometrical models of baufix ${ }^{\circledR}$ objects. baufix ${ }^{\circledR}$ assemblies are represented as collections of elementary objects where the relations among mating features (or ports as the authors call them) are detailedly registered. COAR distinguishes concepts describing objects (e.g. bolt or bar) and concept for roles (e.g. axis or rotor blade) which are related by corresponding role-of links. Frame like structures are used to model hierarchies of semantical part-of structures for complex real world objects. An airplane, for instance, could consist of a fuselage, wings and other parts where each of the parts might be assembled from simpler units itself. COAR also contains a formalism to represent prototypical shapes of real world objects called imaginal prototyping. On different levels of geometrical abstraction this mechanism analyzes geometric assembly models and extracts symbolic meaning. Hence, equipped with a text processing interface the system realized by Wachsmuth, Jung and Hoffhenke provides a virtual constructor. A user can initiate assembly tasks by typing corresponding natural language commands. Due to its comprehensive knowledge base, the system is able to relate terms from the world of airplanes to baufix ${ }^{\circledR}$ assemblies if they are introduced by the user. Because of its knowledge of prototypical shapes the system can recognize certain assemblies of baufix ${ }^{\circledR}$ objects to represent real world entities. 


\subsection{Visual Assembly Process Monitoring}

Automatic assembly monitoring is a research topic of increasing significance; issues of interest reach from quality control and process monitoring to sequence learning by demonstration. Literature on assembly observation is rich, however, although vision solutions exist, computer vision does not yet play a predominant role in this field of application.

Noble [93] addresses the industrial usability of computer vision in principle. She describes the state of the art in geometry driven vision inspection and enumerates critical issues and open problems. As a major challenge in vision for manufacturing she identifies the need for tolerant object models. Noble argues that it is cumbersome to always provide up-to-date models and that these usually are idealized and thus may lead to acceptable parts being rejected by vision based inspection algorithms. Therefore, she proposes to apply stochastic templates which average a number of prototypical object views and demonstrates the suitability of this idea by means of examples. A study performed by Nelson, Papanikolopoulos and Khosla [89] points in the same direction. The authors examine possibilities to integrate visual servoing into robotic manufacturing. Their motivation is to increase the robustness of assembly systems that are programmed based on geometrical assembly models. By means of visual feedback from static or dynamic cameras they realize a control loop to track objects in an assembly cell and thereby facilitate the handling of misplaced parts. An example for machine vision in quality control for assembly is presented by Khawaja et al. [66]. Given geometric models they generate synthetic images of tooth wheels which are used to train a vision system for error inspection in an application concerned with simple gearboxes.

Another issue of interest in automatic assembly monitoring is execution observation. Methods to verify that individual assembly tasks or whole assembly plans are executed correctly have been proposed by several researchers. Many of them make use of computer vision techniques, however, vision usually is not the only source of information in execution monitoring. Syed, ElMaraghy, and Chagneux [124], for instance, apply Kohonen maps to classify sensory data yielded by a camera and several force sensors. Their objective is to verify in real time whether robotic manipulators correctly approach objects that have to be grasped. A knowledge based approach to assembly diagnosing is presented by Abu-Hamdam and Sherif El-Gizawy [2]. They define a taxonomy of errors that might occur before and during an assembly task. Two expert systems are coupled via a blackboard architecture; one is responsible for error detection while the other accomplishes error diagnosis. Sensory input to the experts is provided by collection of devices including a camera, tactile arrays and force-torque sensors.

Several articles by Ikeuchi and his coworkers $[60,61,62,85,86]$, work by Tung and Kak [129] and contributions by Dillmann and colleagues [31, 47] extend the use of vision in assembly from quality and execution control to process understanding and learning by demonstration. They all introduce systems that analyze assembly tasks demonstrated by a human and derive corresponding plans and manipulator trajectories. Again, similar 

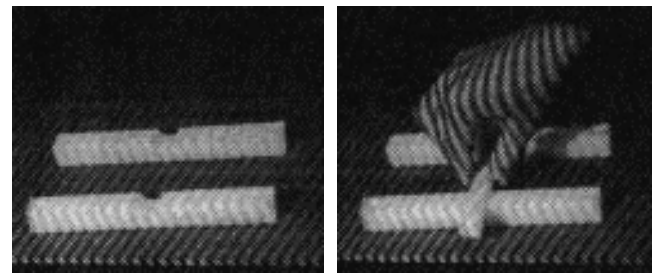

(a) Observation of an assembly task

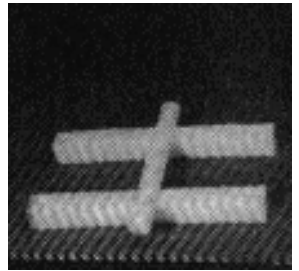

(b) Geometric object model

Figure 2.3.: Examples from a paper by Ikeuchi, Kawade, and Suehiro [60]. The images show stages of an assembly task that are evaluated automatically and lead to the geometric model depicted on the right. (Pictures (C)1993 IEEE).

to the work on execution control outlined above, none of the contributed methods purely relies on vision.

Figure 2.3 shows images found in a publication by Ikeuchi, Kawade, and Suehiro [60]. On the left, a sequence of images is shown which depicts the course of an assembly task. Based on sequences like this Ikeuchi and his coworkers extract actions performed by a human and generate corresponding manipulator actions. A camera permanently provides grey value images of the construction cell. Whenever there are significant differences between consecutive images, an assembly task is assumed to take place. If the differences between images become negligible again, the task is supposed to be over and a laser range finder is used to determine which objects have been manipulated and how they are situated. To this end geometric models of individual objects are fitted into the highly accurate range data. This step results in geometrical assembly models as shown in Fig. 2.3(b). By means of a predefined taxonomy of surface contacts the surface relations within reconstructed assemblies are classified. As each surface contact in the taxonomy is associated with a sequence of manipulator actions, it is possible to derive manipulator sequences reproducing the observed actions. Moreover, suitable grasping points are estimated from difference images as well. They are assumed to be situated at points of intersection between the geometric representation of the manipulated object and a model of hand fitted into the camera images.

Tung and Kak [129] address the integration of sensing, task planning and execution control for assembly. They realized a robot that learns assembly sequences demonstrated by a user. While performing the task, the user wears a data glove so that corresponding manipulator motions can be deduced. After learning an assembly sequence for a set of parts, the system is capable to assemble those parts, no matter how they are positioned in its workspace. Each part is modeled geometrically and as they are of simple shape and carry markers (see Fig. 1.3(a) on page 7), 2D vision is sufficient to estimate their position and orientation. Dillmann and his coworkers [31, 47] also combine different sensing strategies in their implementations of skill acquiring systems. Like Tung and 
Kak they process data provided by a data glove and a camera. Again the camera is used for object localization while the data glove serves to reconstruct manipulator motions. Using this integration scheme their systems is able to perform simple pick and place operations, bag packing and the assembly of simply shaped parts.

Recently, augmented reality methods have been applied to the learning by demonstration paradigm as well. Freund and Rossmann [37] describe how to project virtually demonstrated assembly processes into real world assembly cells and present several applications of this technique. Among the advantages offered by this approach the authors esteem applications in remote control and intuitive robot programming to be the most promising ones. A similar concept is introduced by Lloyd et al. [78] who also aim at virtual demonstration in assembly. Their system only copes with marked block world objects (see Fig. 1.3(b) on page 7), however, it is equipped with a 3D vision device so that realistic environment models can be constructed from the physical surroundings. Raghavan, Molineros, and Sharma [101] describe an interactive tool for support manual assembly. While assembling certain parts, a user observes the environment through a head display and useful information is projected into the scene. Again, object recognition is done by means of geometric models and facilitated by markers attached to the objects.

\subsection{Mathematical Complexity}

Most of the approaches to assembly modeling we surveyed in this chapter were found in contributions concerned with assembly sequence planning. This does not surprise since in industrial manufacturing there is considerable demand for optimal assembly sequences ${ }^{3}$. Likewise it is not surprising that several researches have examined the problem of assembly sequence planning in principle. They asked whether optimal sequences can be found in any case or if there are assemblies where, due to the expected computational effort, one has to be contend with suboptimal solutions provided by heuristic methods. Due to its practical impact we will shortly summarize results concerning this issue, i.e. the mathematical complexity of assembly modeling and planning.

Many contributions to automatic assembly planning presume geometric assembly models that can be separated using methods from computational geometry in order to search for optimal sequences. However, Kavraki and her colleagues [65] remind that for 3D objects this is an $N P$ complete problem (cf. [88]) and moreover prove that even planar partitioning into connected subassemblies is $N P$ complete. Formally, they study the question if, given a set $A$ of non-overlapping polygons in the plane, a proper subset $S \subset A$ can be separated from a $A \backslash S$ by a collision free rigid motion of $S$ where both $S$ and $A \backslash S$ are connected. The $N P$ completeness of this problem is proven by reduction from the well known $N P$ complete problem called 3SAT. Goldwasser, Latombe, and

\footnotetext{
${ }^{3}$ In industrial practice optimal is usually synonymous with minimal manufacturing costs.
} 
Motawi [46] reach the same result by reducing the problem of collision free part removal from the NP complete SET COVER problem. Being aware of the complexity of assembly planning Ramos, Rocha and Vale [102] explain that it is neither caused by the number of necessary assembly tasks nor by the number of components but by assembly topology and they present an algorithm to determine the exact number sequences from a given precedence graph without counting them. Homem de Mello and Sanderson [55] also investigate how assembly topology influences the complexity of the planning problem. Interested in the storage requirements of their AND/OR graph approach they empirically derive an average of $O\left(2^{n}\right)$ for assemblies of $n$ strongly interconnected components.

Again in terms of computer memory Wolter [136] examines the costs of several modeling methods. For each method he considers different assembly topologies and derives upper bounds for the size of the corresponding data structures. Except of some special cases the storage requirements of all inspected methods grow exponentially. AND/OR graphs, however, prove to be the most compact way to represent assembly plans.

\subsection{Discussion}

Over the last decade there were extensive research activities dedicated to assembly modeling. These were mainly stimulated by two rather antipodal interests. On the one hand, economical demands from industry led to research in automatic assembly sequence planning and a lot of modeling techniques were proposed in this discipline. On the other hand, progress in basic research on intelligent robotics motivated scientists to explore intelligent approaches to ever more complex tasks and assembly modeling techniques emerged from studies in process understanding or learning by demonstration.

In this chapter we reviewed various contributions to assembly modeling and saw that there are two basic paradigms. Geometry based approaches provide detailed information of physical dimensions of elementary objects and assemblies are represented as feasible unions of elementary models. Structural modeling captures hierarchical, topological or any other sort of semantically relevant relations among assembled parts. There also are hybrid approaches combining geometrical and structural information. Structural models, for example, might represent geometric relations among parts and geometric models might be augmented with information of, for instance, the function of a part.

As this thesis is concerned with assembly recognition from computer vision it remains to verify to what extend these paradigms and the specific approaches outlined above are suited for our problem. In the following we shall therefore assess the advantages and disadvantages of the different approaches. In doing this we must remember that we are dealing with a scenario of cooperative and flexible assembly where the instructor's choice what to construct should be as less restricted as possible.

Structural approaches to assembly modeling usually provide simple models of individual assemblies. They abstract from geometry but might represent any other kind of 
relevant relations or information. In general, the components and relations of a structural model are chosen with regard to a specific application. A major drawback, however, is that structural models seldom are generic. Model acquisition usually takes an expert who has to edit the necessary information. Furthermore, except for a contribution by Stadzisz and Henrioud [121], which we will discuss later, no ideas how to generalize structural assembly models were reported. Thus, assembly recognition based on structural models would imply considerable modeling effort, if more than just a few assemblies should be recognized. Recognizing any feasible assembly by means of individual models is obviously impracticable. Conscious of the acquisition problem, many contributors describe how to compute structural models from representations like, for instance, geometrical models. But this evidently only shifts the problem to the supply of large sets of geometric models and does not provide a generic solution.

Geometrical assembly modeling requires to represent physical dimensions of elementary objects and a collection of rules to describe well formed object compositions. Thus, geometrical assembly modeling is more generic than most structural approaches: composition constraints can be implemented using methods from computational geometry which enables to generate assembly models automatically. Likewise, the composition rules allow to test if an arbitrary configuration of objects depicts a feasible assembly. Due to their generic nature, geometric modeling techniques seem appropriate for recognition and have actually been applied to assembly process monitoring. However, extracting exact geometric information from image data is burdensome and computationally expensive. Consequently, as the examples in Fig. 1.3 and Fig. 2.3 on pages 7 and 21 substantiate, even recent approaches to assembly observation only deal with simple block world objects. Moreover, they either require precise measurements like provided by laser range finders or use markers to facilitate recognition and pose estimation. This, of course, is a severe restriction that we would like to avoid in our scenario.

Apart from structural and geometrical modeling, one can think of implicit knowledge representation for assembly recognition. Some researchers proposed the use of templates for assembly monitoring. This idea, however, suffers from the same drawback like many structural methods: realizing a flexible assembly recognition system would require numerous templates even if statistical templates were used. Nevertheless, assemblies or, even worse, views of an assembly not represented by a (statistical) template could not be recognized.

In our discussion of assembly modeling techniques within the SFB 360 we described a grammar based approach by Rieser and Meyer-Fujara [107]. Formal grammars usually generate large sets of entities and thus provide a compact way to represent structural knowledge of whole object classes. However, the grammars proposed by Rieser and Meyer-Fujara model individual assemblies and do not enable flexible recognition. But the set generating properties of grammars are interesting. If it was possible to generalize from grammars describing individual objects, a generic representation of assembly knowledge would be available. 
Stadzisz and Henrioud [121] gather liaison graphs of a family of products and derive web grammars. They claim their approach to be generic and present an exemplary grammar which describes different products. However, all the products are topologically equivalent, i.e. they do have the same component structure. Thus, the grammar basically enumerates feasible permutations of elementary objects. It is a collection of individual models and does not describe abstract composition principles. Viewed from a formal grammar perspective, its main shortcoming is that Stadzisz and Henrioud do not make use of the mighty principle of recursion. Therefore, with respect to flexible recognition this approach, too, is inappropriate.

Summarizing the preceding paragraphs we see that it is possible to model assemblies by means of formal grammars which are known to be a compact method to represent knowledge of huge sets of entities with a certain structure. If it was possible to identify abstract principles behind the component structure of a class of assemblies, we should be able to provide grammars that generate objects whose internal structure obeys these principles. Likewise, by means of grammatical parsing we had a mechanism to decide whether an arbitrary object belongs to the class of assemblies modeled by the grammar. Therefore, grammars seem predestined to model assembly knowledge in an assembly recognition application. However, we must be aware of the complexity of the assembly partitioning problem: with a growing number of parts an assembly can be separated into a growing number of subassemblies which again can be structured in numerous different ways. Generally, this will cause grammatical models to derive many different descriptions of an individual assembly which of course are semantically equivalent. Rieser and Meyer-Fujara observed this phenomenon even in the case of simple assemblies and called it spurious ambiguities. This indicates that individual assembly recognition can hardly be performed by means of grammatical models alone and that it will require further thought. 


\section{Syntactic Assembly Modeling}

Mechanical assembly is a process of constructing complex objects from a set of separate parts. It results in composite units whose individual components are physically connected to at least another one. Mechanical assembly thus imposes structure on a set of unrelated parts. It actually yields several structures because assembly establishes different relations among the parts and each relation may serve as a criterion to structure the set; common examples for such criteria are the relations is adjacent to and consists-of.

Since assembly components may be elementary or composite objects, the consistsof criterion normally reveals hierarchical structures where higher-level parts consist of lower-level ones which again may have internal structure. In the following, we shall investigate this phenomenon more closely. We will introduce context free grammars to represent and analyze hierarchical assembly structures and we will apply them to visual assembly recognition. This framework is not restricted to baufix ${ }^{\circledR}$ assemblies but the toy objects will help to set forth our ideas. In preparation for this we will first present further general observations.

Generally, assemblies consist of multifunctional objects which might form numerous other assemblies. Yet not any set of parts can be assembled into a stable unit. Mechanical assembly rather means to rigidly connect parts via surface features such that only marginal relative motion is possible. Therefore, the parts must show surface features that enable interconnections and simultaneously reduce the degree of freedom for mutual motion. These mating features are well known in assembly research where their geometrical appearance is of primary concern (cf. e.g. [130]). We, however, will consider their function: from an abstract point of view an assembly consists of several functional units each providing necessary features to realize a stable connection. Simultaneously, an assembly is composed of several subassemblies that may be elementary parts or assembled objects. Thus, from a functional viewpoint elementary parts and assemblies are equivalent. They both must provide mating features to be assembled.

A feasible assembly consists of a finite set of elementary components and each component has a finite number of mating features. In most practical cases, however, the number of different mating features found within an assembly is low and there are few disparate but many similar attachments. Assemblies can thus be classified according to the predominant attachment; baufix ${ }^{\circledR}$ assemblies, for example, belong to the class of bolted assemblies.

Bolted connections where a bolt and a nut hold together a series of parts are a 


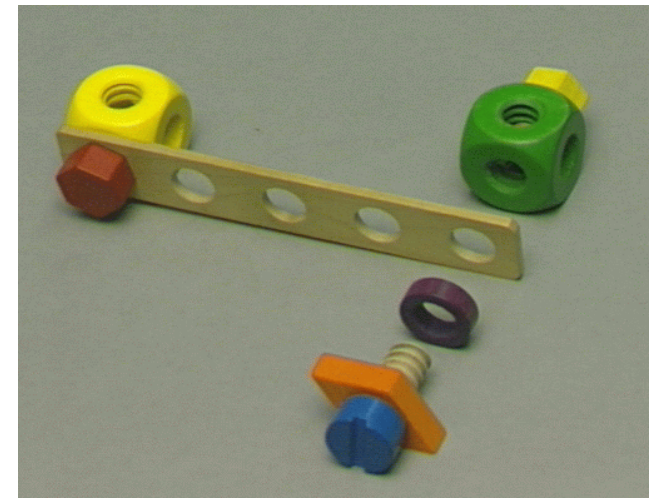

(a)

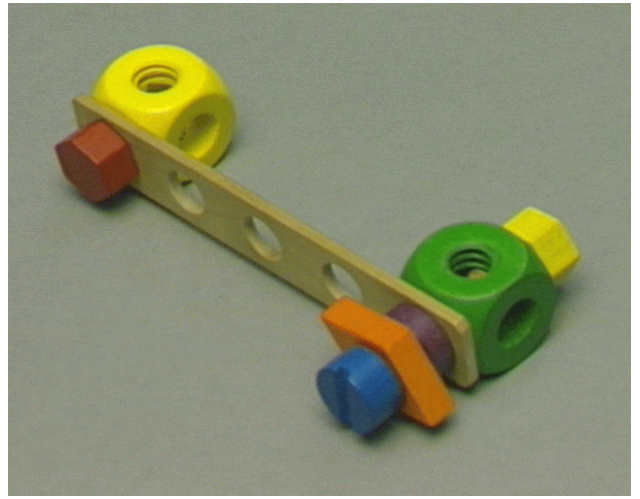

(b)

Figure 3.1.: Illustration of the basic principles of constructing bolted assemblies.

common mechanical structure and draftsmen know various techniques to establish rigid connections between bolts and nuts. Screw connections between threaded cylindrical shafts and holes, however, are probably the most common method.

baufix ${ }^{\circledR}$ assemblies are bolted assemblies with screw connections. A baufix ${ }^{\circledR}$ bolt has a threaded shaft while cubes and rhomb-nuts provide threaded holes. Apart from bolts and nuts there are further baufix ${ }^{\circledR}$ parts which we will call miscellaneous objects in the following. Miscellaneous objects like bars or rings have simple cylindrical holes and can be put onto bolts. However, unless there is a fixating nut, connections of bolts and miscellaneous objects will not be stable. Figure 3.1 depicts prototypical feasible assemblies (or feature combinations) realizable with baufix ${ }^{\circledR}$ parts. From these examples we can conclude three basic principles for the construction of baufix ${ }^{\circledR}$ assemblies:

1. A baufix ${ }^{\circledR}$ assembly consists at least of a bolt and a nut.

2. Optionally, these parts may fix some miscellaneous objects.

3. All components are either elementary objects or assemblies.

Together with our observations concerning the inherent function of assembly components, these principles directly lead to the abstract model of bolted assemblies shown in Fig. 3.2. The model summarizes that a baufix ${ }^{\circledR}$ assembly consists of two obligatory and a couple of optional parts. To stress their functional significance the parts are termed correspondingly. The Bolt-Part provides a threaded shaft, the Misc-Parts must provide simple holes, and the Nut-Part has to contribute a threaded hole. Since elementary components and subassemblies are functionally equivalent, the model also involves recursion: each functional part may be an elementary object or an assembly. 


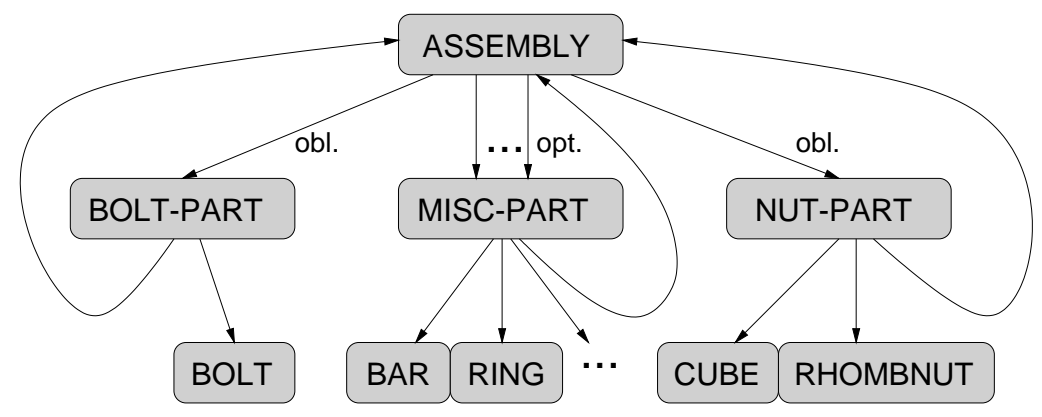

Figure 3.2.: Recursive model of the principle structure of bolted assemblies.

As it makes use of recursion, the model provides a very compact representation of abstract assembly knowledge. It enables to describe the mechanical structure of any baufix ${ }^{\circledR}$ assembly; the assembly in Fig. 3.1(b), for instance, can be structured as follows:

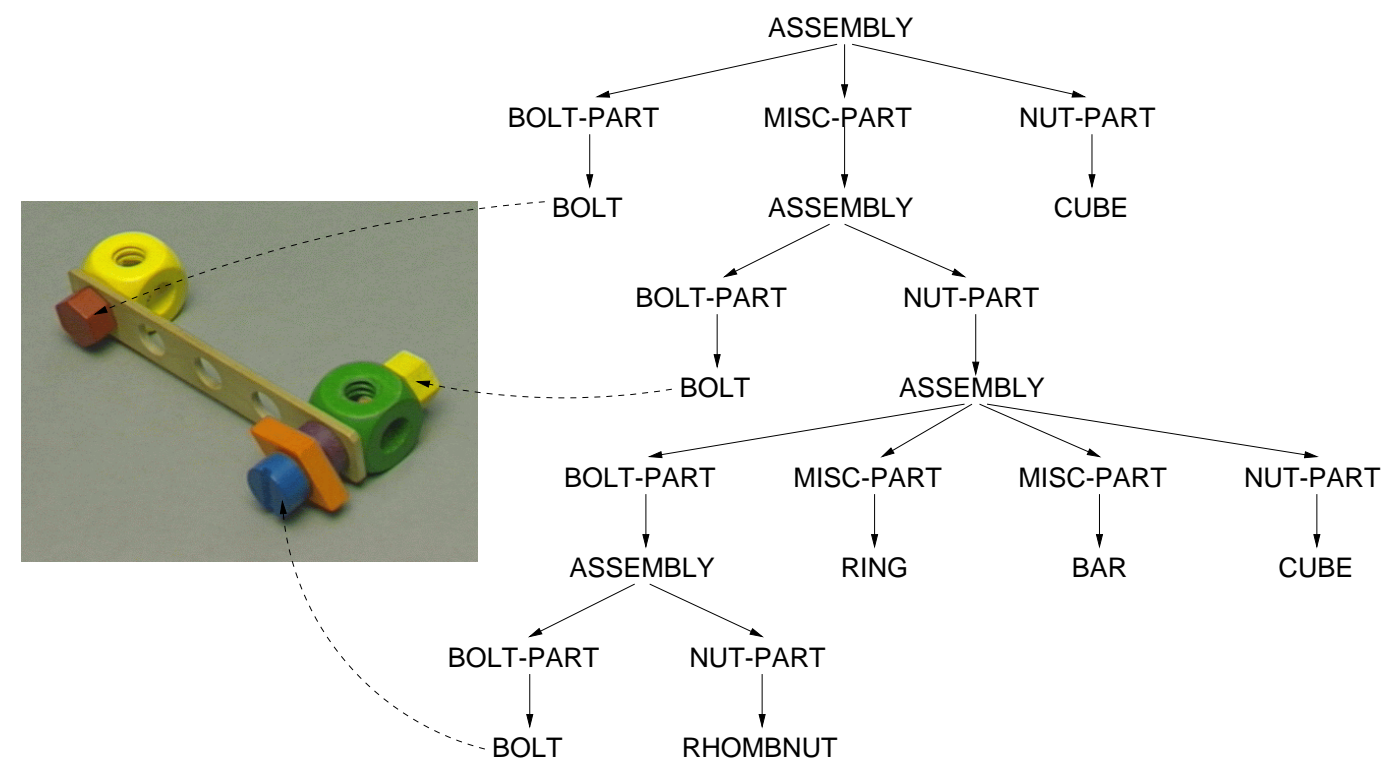

Figure 3.3.: A possible hierarchical description of the structure of the assembly in Fig. 3.1(b), derived according to the above model. Dashed arrows indicate correspondences between hierarchical levels and parts of the assembly.

This structure describes the assembly as a composition of a (red) bolt, a complex miscellaneous part, and a (yellow) cube. The miscellaneous part is an assembly of a (yellow) bolt and a nut which consists of a complex bolt, a ring, a bar, and a (green) cube. Finally, on the lowest level of the hierarchy the complex bolt is represented by a (blue) bolt and a rhomb-nut. Obviously, this hierarchy is not the only one to characterize the 
assembly. It would, for example, be perfectly acceptable to represent it as a combination of a (yellow) bolt and a corresponding complex nut. In fact, the selection of objects itemized on the highest level of the hierarchy is almost arbitrary while the objects on the levels below will depend on this choice. If we listed all possible choices, there would be five different hierarchical structures all equally suited to characterize the assembly. We will return to this later.

Simple considerations regarding the function of subassemblies or the functional meaning of mating features thus lead to a simple, generic model of the class of bolted assemblies which allows to generate structural descriptions of any baufix ${ }^{\circledR}$ assembly. These descriptions abstract from geometric details but reflect structures due to consists-of relations among subsets of parts of an assembly. Next, we shall see that this generic modeling approach is closely related to formal language theory.

\subsection{Formal Language Theory and Assembly Modeling}

The notion of formal languages was first introduced by Chomsky [27]. A formal language $L$ is a set of words over an alphabet $T$, i.e. $L \subseteq T^{*}$. The elements of $L$ are selected by a finite set of rules called a formal grammar. A tuple $G=(N, T, P, S)$ defines a Chomskygrammar if

$N$ is an alphabet of non terminal symbols (also called variables)

$T$ is an alphabet of terminal symbols where $N \cap T=\emptyset$

$P \subseteq\left((N \cup T)^{*} \cdot N \cdot(N \cup T)^{*}\right) \times(N \cup T)^{*}$ is a non empty set of productions

$S \in N$ is a variable called the start symbol.

To denote that $(u, v)$ is a production in $P$ we write $u \rightarrow v$. If $u \rightarrow v$ is a production in $P$ and $a$ and $b$ are strings in $(N \cup T)^{*}$ we write $a u b \Rightarrow a v b$ and say that $a u b$ directly derives $a v b$. If $a_{1}, a_{2}, \ldots a_{n}, n \geq 1$, are strings in $(N \cup T)^{*}$ and $a_{1} \Rightarrow a_{2}, a_{2} \Rightarrow a_{3}, \ldots$, $a_{n-1} \Rightarrow a_{n}$ we write $a_{1} \stackrel{*}{\Rightarrow} a_{n}$ and say that $a_{1}$ derives $a_{n}$. Given this terminology, the formal language $L(G)$ generated by a grammar $G$ is defined as follows:

$$
L(G)=\left\{x \in T^{*} \mid S \stackrel{*}{\Rightarrow} x\right\}
$$

In his work on linguistic structures Chomsky identified different classes of formal languages with different mathematical properties. Languages are usually classified with regard to the productions of their generating grammars. In the above definition we presented the most general type of grammar that generates the so called type- 0 or unrestricted languages. Formal languages of other classes are derived from grammars with less flexible productions. In the following we will narrow our discussion to the class of type-2 or context free languages and to the corresponding context free grammars (CFGs). 
A CFG is a Chomsky-grammar characterized by productions $u \rightarrow v$ with $u \in N$ and $v \in(N \cup T)^{*} \backslash\{\epsilon\}$ where $\epsilon$ denotes the empty word.

Context free grammars are of considerable importance in computer science. An important application lies, for instance, in the theory of programming languages. If a CFG is used to specify a programming language, there will be a mechanism called parsing that verifies whether a given program is syntactically correct, i.e. whether it is a word of the corresponding language. This thesis will promote the idea that CFGs also provide a means to model individual mechanical assemblies as well as classes of assemblies. We will see that any assembly can be syntactically structured according to a CFG. This indicates the existence of parsing algorithms to check if a given configuration of parts corresponds to a feasible assembly which might be useful for vision purposes. Before we generally discuss these topics let us motivate the idea by means of an example.

\subsubsection{A Context Free Grammar of baufix ${ }^{\circledR}$ Assemblies}

The abstract model of the class of baufix ${ }^{\circledR}$ assemblies presented above corresponds to a context free grammar $G=(N, T, P, S)$ with the following set of variables

$$
N=\{\text { ASSEMBLY, BOLT-PART, MISC-PART, NUT-PART }\},
$$

the set of terminals

$$
T=\{B O L T, B A R, R I N G, F E L L Y, S O C K E T, C U B E, R H O M B N U T\},
$$

the start symbol $S=$ ASSEMBLY and the productions $P$ chosen to be:

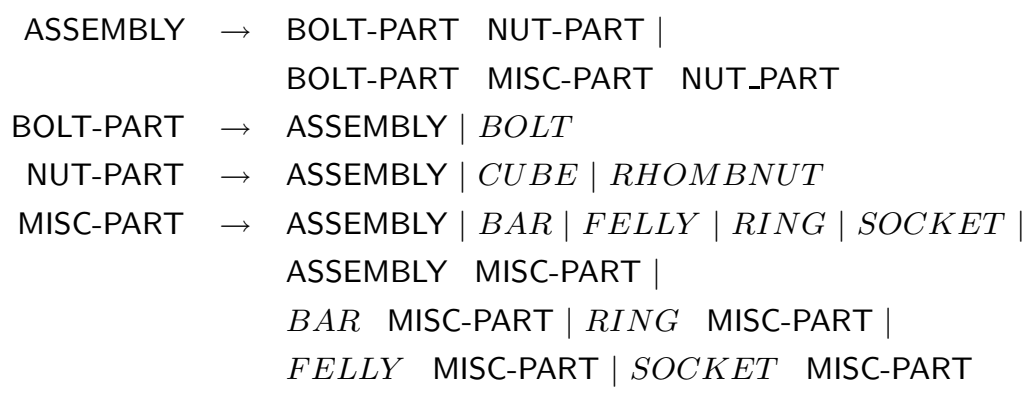

Elementary baufix ${ }^{\circledR}$ parts correspond to terminal symbols of this grammar while assemblies and their functional parts appear as variables. The productions 3.1 and 3.2 describe the composition of a bolted assembly and point out that the miscellaneous-part is optional. The next productions state that the bolt- and the nut-part either consist of a (sub)assembly or of an elementary object. Rules 3.5-3.8 describe how the miscellaneouspart of an assembly is structured: it either is a single part or a subassembly or a sequence of those. 
Note that the language $L(G)$ generated by this grammar also contains words not corresponding to mechanically feasible assemblies. For instance, there are words composed of numerous bolts and a single cube which evidently are infeasible. Or, reconsider the rules $3.5-3.8$ which slightly differ from the model in Fig. 3.2. In the grammar, the fact that there might be sequences of miscellaneous objects is expressed using recursion. But recursive productions do not specify how many objects there might be at most ${ }^{1}$. In reality, however, the number of possible miscellaneous objects is constrained by the length of the bolt to which they are attached. Thus, as the grammar cannot express context sensitive mechanical restrictions like that, the set of words corresponding to feasible baufix ${ }^{\circledR}$ assemblies will only be a small subset of $L(G)$. For the time being, we will postpone this problem but we shall return to it in Chapter 3.2.

Like every CFG this simple grammar produces linear structures. And even though sophisticated syntactic methods like graph grammars seem more appropriate to represent 3D assemblies, we shall see that this CFG is sufficient for our purposes. Moreover, context free grammatical models are neither restricted to assemblies with a single type of mating feature combination nor to domains with binary mating relations. In [11] we presented rather didactic examples to illustrate that the principles deduced for bolted assemblies can be generalized to n-ary mating relations and several types of connections. Actually, the existence of context free grammars that model composite objects is not an accident but an inherent property of mechanical assemblies which is due to the fact that they can be structured according to the consists-of relation. Each hierarchical structure of an assembly corresponds to a word of at least one context free language. Furthermore, as we will explain next, there is a general mechanism to generalize and combine different context free languages associated with different assemblies such that grammars modeling whole classes of assemblies can be derived from examples.

\subsubsection{Learning Assembly Grammars from Examples}

In Chapter 2, we discussed Homem de Mello's and Sanderson's [53, 54, 55] approach to knowledge representation for assembly tasks. Assuming detailed relational models to be given, they presented an algorithm to decompose assemblies and proved it to be complete and correct. I.e. they proved that their algorithm generates all feasible decompositions of an assembly. To represent the resulting assembly sequences the authors proposed the use of AND/OR graphs which, as a comparative study by Wolter [136] yielded, is in fact the most efficient way to store assembly sequence data. Therefore, it is no surprise that AND/OR graphs have become a standard in assembly modeling. However, already almost 30 years ago Hall [49] noted that there is a one to one correspondence between AND/OR graphs and context free grammars. The example in Fig. 3.4 should illustrate

\footnotetext{
${ }^{1}$ Neither does the above model. Thus, both methods are equally appropriate to model arbitrary sequences of miscellaneous objects
} 


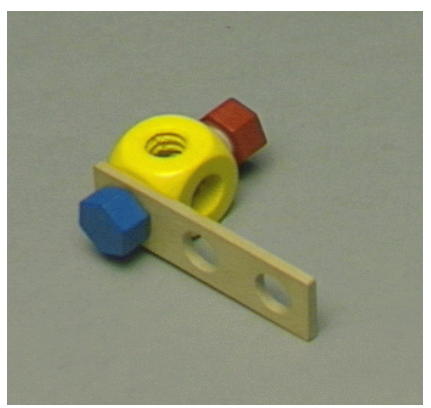

(a)

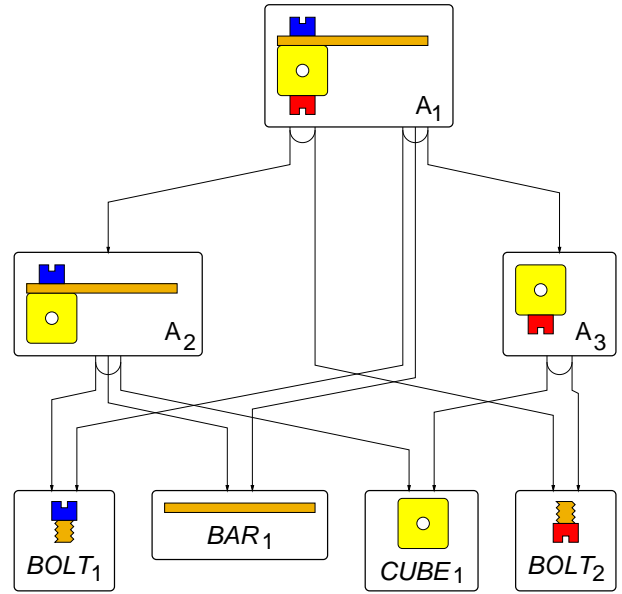

(b)

Figure 3.4.: An exemplary assembly and its feasible decompositions represented as an AND/OR graph.

this. The depicted an AND/OR graph for the exemplary assembly corresponds to a CFG $G_{\mathrm{A}}$ with

$$
G_{\mathrm{A}}=\left(N_{\mathrm{A}}=\left\{\mathrm{A}_{1}, \mathrm{~A}_{2}, \mathrm{~A}_{3}\right\}, T_{\mathrm{A}}=\left\{B O L T_{1}, B O L T_{2}, C U B E_{1}, B A R_{1}\right\}, P_{\mathrm{A}}, S_{\mathrm{A}}=\mathrm{A}_{1}\right)
$$

where $P_{\mathrm{A}}$ contains the following productions:

$$
\begin{aligned}
& \mathrm{A}_{1} \rightarrow B O L T_{2} \mathrm{~A}_{2} \mid B O L T_{1} B A R_{1} \mathrm{~A}_{3} \\
& \mathrm{~A}_{2} \rightarrow B O L T_{1} B A R_{1} C U B E_{1} \\
& \mathrm{~A}_{3} \rightarrow B O L T_{2} C U B E_{1} .
\end{aligned}
$$

Hence, nodes with descending hyperlinks are mapped onto grammatical variables, leaf nodes are equal to terminal symbols, and hyperlinks correspond to the productions of a grammar. The assembly and its complex subassemblies thus appear as the variables of $G_{\mathrm{A}}$ while its elementary components are the terminal symbols; the language derivable from $G_{\mathrm{A}}$ is

$$
\begin{aligned}
L\left(G_{\mathrm{A}}\right) & =\left\{x \mid \mathrm{A}_{1} \stackrel{*}{\Rightarrow} x\right\} \\
& =\left\{B O L T_{2} B O L T_{1} B A R_{1} C U B E_{1}, B O L T_{1} B A R_{1} B O L T_{2} C U B E_{1}\right\} .
\end{aligned}
$$

Each tree of the AND/OR graph can thus be identified with a word of the grammar. Consequently, hierarchical component structures of an assembly can be represented as linear sequences of symbols ${ }^{2}$.

\footnotetext{
${ }^{2}$ Hall pointed out that mapping an AND/OR graph to a grammar imposes an ordering on the edges of the hyperlinks but argued that this is no loss of generality and that both techniques are equivalent,
} 
Since such sequences strongly abstract from geometric details and relations among individual mating features and since the same set of objects might be assembled into geometrically different configurations with yet the same component structure, the language actually characterizes a set of assemblies ${ }^{3}$. It is an inherent feature of each assembly in this set. And as any mechanical assembly can be structured by means of an AND/OR graph for which a corresponding CFG can be specified, there is a characteristic context free language for any assembly.

Our exemplary grammar, however, only describes a small set of structurally equivalent assemblies. It does, for instance, not derive words characterizing the complex subassemblies $A_{2}$ and $A_{3}$ of the underlying assembly. But it is possible to generalize it such that it will characterize a whole class of assemblies. In fact, the following four step procedure will do the trick:

1. Express the fact that assemblies are composed of functional units. Introduce functional layers to the grammar, i.e. add functional variables and corresponding productions.

2. Identify the functional significance of the new variables and further generalize the grammar by substituting corresponding symbols for them.

3. Extrapolate from syntactic relations among individual objects to relations among object types. I.e. replace terminals denoting individual objects by terminals denoting object types.

4. Further generalize the grammar by introducing recursive productions. To this end substitute a general assembly symbol for all variables denoting complex objects.

Presented on a level of detail like this, executing this algorithm seems to require a human expert. However, if we assume the assembly to be modeled accordingly, the generalization of the grammar can be accomplished automatically. To see this, we shall sketch the above steps in more detail.

1. Introduction of functional layers: Add new symbols to the set of variables $N_{\mathrm{A}}$ such that for each symbol $a \in N_{\mathrm{A}} \cup T_{\mathrm{A}}$ that appears on the right hand side of a production there will be a variable $\mathrm{f}(a)$. For our example this will yield six new variables $\mathrm{f}\left(\mathrm{A}_{2}\right), \ldots, \mathrm{f}\left(C U B E_{1}\right)$.

For each of the new variables introduce productions $\mathrm{f}(a) \rightarrow a$ and replace old productions $l \rightarrow r_{1} \ldots r_{n}$ by new ones of the form $l \rightarrow \mathrm{f}\left(r_{1}\right) \ldots \mathrm{f}\left(r_{n}\right)$. Here, this will lead to the CFG $G_{\mathrm{A}}^{\prime}=\left(N_{\mathrm{A}}^{\prime}, T_{\mathrm{A}}, P_{\mathrm{A}}^{\prime}, S_{\mathrm{A}}\right)$ visualized in Fig. 3.5.

anyway. We adopt his point of view and will henceforth treat AND/OR graphs and CFGs as equivalent mathematical formalisms; in particular we will use AND/OR graphs as graphical representations of CFGs.

${ }^{3}$ See Appendix A.1 for a more detailed discussion. 


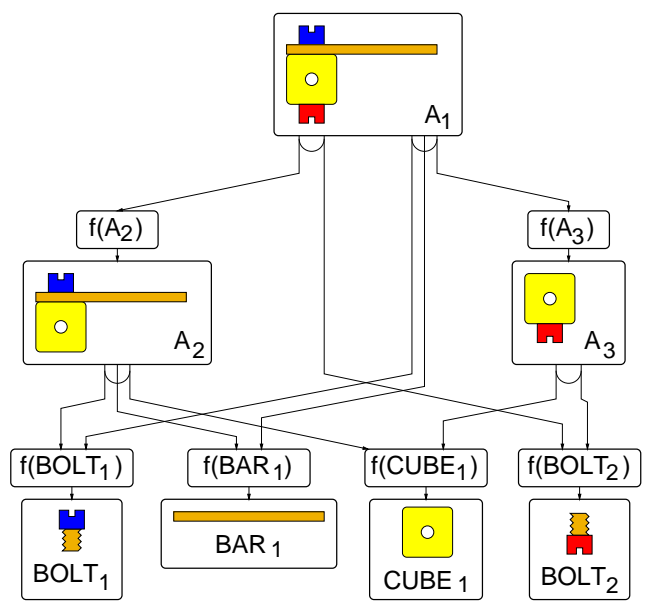

Figure 3.5.: The AND/OR graph with additional nodes $f\left(A_{2}\right), \ldots, f\left(C U B E_{1}\right)$.

Altogether, this step seems unusual for it introduces unnecessary productions whereas in formal language theory interest normally lies in eliminating those (cf. $[45,57])$. However, it will help to integrate our insight concerning the functional meaning of assembly components into the productions of the grammar (remember that the grammar describing all baufix ${ }^{\circledR}$ assemblies also has an additional layer of variables).

2. Identification of functional meaning: Given a suitable representation of (sub)assemblies and elementary objects the functional significance of the new symbols $\mathrm{f}(a)$ can be estimated automatically, too. For our purpose, it suffices to associate sets of mating features to those nodes of the modified AND/OR graph that represent (composite) objects.

Hence, let us assume the assembly represented by the start symbol $S$ comes along with a set

$$
F_{S}=\left\{f_{1}, f_{2}, \ldots f_{n}\right\}
$$

of mating features. Each feature $f \in F_{S}$ belongs to an elementary object (represented by a terminal symbol $t \in T_{\mathrm{A}}$ ) and has a function and a state. Thus, there are three functions $\tau: F_{S} \rightarrow T_{\mathrm{A}}, \varphi: F_{S} \rightarrow \Phi$, and $\sigma_{S}: F_{S} \rightarrow \Sigma$ assigning terminals, functions, and states to mating features in $F_{S}$.

In our example, $\Phi=\{\mathrm{BP}, \mathrm{MP}, \mathrm{NP}\}$ would be a good choice to represent the functions of mating features. It contains abbreviations of bolt-part, misc-part, and nutpart, respectively and thus lists all feature types that occur in the baufix ${ }^{\circledR}$ domain. 
And choosing $\Sigma=\{1,0\}$ will enable to express whether or not a feature participates in an attachment. Hence, mating features can be characterized by a triples like, for instance, $\left(B A R_{1}, \mathrm{MP}, 1\right)$.

Furthermore, let each other symbol $a \in N_{\mathrm{A}} \cup T_{\mathrm{A}}$, that represents a (complex) subassembly be associated with a corresponding set of mating features $F_{a}$ as well. Note that for each $a$ we will have $F_{a} \subseteq F_{S}$ and that the functions $\tau$ and $\varphi$ are the same as for $F_{S}$. This is because terminal symbol and function of a mating feature $f \in F_{a}$ do not depend on $a$. However, the state of a feature certainly will depend on the mechanical context of the object it belongs to. Thus, its state will depend on its location in the AND/OR graph so that for each $a \in N_{\mathrm{A}} \cup T_{\mathrm{A}}$ there is a unique function $\sigma_{a}: F_{a} \rightarrow \Sigma$. Let

$$
\Sigma_{1}(a)=\left\{f \in F_{a} \mid \sigma_{a}(f)=1\right\} \quad \text { and } \quad \Sigma_{0}(a)=\left\{f \in F_{a} \mid \sigma_{a}(f)=0\right\}
$$

be subsets of mating features associated to $a$ containing those features that are or are not part of an attachment, respectively. Finally, if $a_{i} \rightarrow \mathrm{f}\left(a_{j}\right)$ and $\mathrm{f}\left(a_{j}\right) \rightarrow a_{j}$ are productions in the extended grammar, we define

$$
\operatorname{prec}\left(\mathrm{f}\left(a_{j}\right)\right)=a_{i} \quad \text { and } \quad \operatorname{succ}\left(\mathrm{f}\left(a_{j}\right)\right)=a_{j} .
$$

Given all this, the functional significance of a variable $\mathrm{f}(a)$ can be determined by evaluating the expression

$$
\Phi(\mathrm{f}(a))=\left\{\varphi(f) \mid f \in \Sigma_{1}(\operatorname{prec}(\mathrm{f}(a))) \cap \Sigma_{0}(\operatorname{succ}(\mathrm{f}(a)))\right\} .
$$

The functional significance of a variable $\mathrm{f}(a)$ thus can be determined from the features that are part of an attachment in its predecessor in the AND/OR graph but are not connected to other features in the succeeding node. I.e. the functional significance of $\mathrm{f}(a)$ and thus of the part in its succeeding node is determined by the functions $\varphi$ of those features that change their state in an assembly task.

Figure 3.6 illustrates this idea. It shows the objects $\mathrm{A}_{2}$ and $C U B E_{1}$ and the corresponding sets of mating features ${ }^{4}$. In this example, we have

$$
\Sigma_{1}\left(\mathrm{~A}_{2}\right) \cap \Sigma_{0}\left(C U B E_{1}\right)=\left\{f_{9}, f_{8}, f_{7}, f_{1}\right\} \cap\left\{f_{4}, f_{3}, f_{2}, f_{1}\right\}=\left\{f_{1}\right\} .
$$

Thus, feature $f_{1}$ of $C U B E_{1}$ changes its state if the cube becomes part of the assembly $\mathrm{A}_{2}$. And the meaning of the functional variable $\mathrm{f}\left(C U B E_{1}\right)$, i.e. the function this variable stands for, is given by the set $\Phi\left(\mathrm{f}\left(C U B E_{1}\right)=\left\{\varphi\left(f_{1}\right)\right\}=\{\mathrm{NP}\}\right.$. We can therefore conclude that the cube is the nut-part of the assembly $\mathrm{A}_{2}$.

\footnotetext{
${ }^{4}$ Note that baufix ${ }^{\circledR}$ cubes supply four threaded holes and that the continous thread of a bolt can be represented by a discrete number of mating features which will depend on its length.
} 


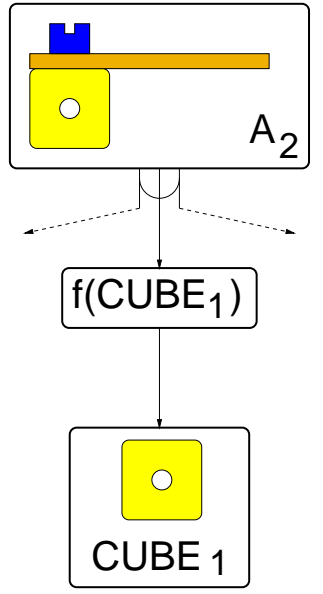

(a)

$$
\begin{gathered}
F_{\mathrm{A}_{2}}=\left\{\begin{array}{rll}
f_{9} & = & \left(B O L T_{1}, \mathrm{BP}, 1,\right) \\
f_{8} & = & \left(B O L T_{1}, \mathrm{BP}, 1\right), \\
f_{7} & = & \left(B A R_{1}, \mathrm{NP}, 1\right), \\
f_{6} & = & \left(B A R_{1}, \mathrm{NP}, 0\right), \\
f_{5} & = & \left(B A R_{1}, \mathrm{NP}, 0\right), \\
f_{4}= & \left(C U B E_{1}, \mathrm{NP}, 0\right), \\
f_{3}= & \left(C U B E_{1}, \mathrm{NP}, 0\right), \\
f_{2}= & \left(C U B E_{1}, \mathrm{NP}, 0\right), \\
f_{1}= & \left(C U B E_{1}, \mathrm{NP}, 1\right)
\end{array}\right\} \\
F_{C U B E_{1}}=\left\{\begin{array}{rll}
f_{4}= & \left(C U B E_{1}, \mathrm{NP}, 0\right), \\
f_{3}= & \left(C U B E_{1}, \mathrm{NP}, 0\right), \\
f_{2}= & \left(C U B E_{1}, \mathrm{NP}, 0\right), \\
f_{1}= & \left(C U B E_{1}, \mathrm{NP}, 0\right)
\end{array}\right\}
\end{gathered}
$$

(b)

Figure 3.6.: A detail of the modified AND/OR graph in Fig. 3.5 and the corresponding sets of mating features.

In order to integrate functional meaning into the grammar, extend the already modified set of variables $N_{\mathrm{A}}^{\prime}$ by the power set $2^{\Phi 5}$. Then substitute the functional significance $\Phi(\mathrm{f}(a))$ for the symbols $\mathrm{f}(a)$ and correspondigly adjust the concerned productions.

This step yields a new CFG $G_{\mathrm{A}}^{\prime \prime}=\left(N_{\mathrm{A}}^{\prime \prime}, T_{\mathrm{A}}, P_{\mathrm{A}}^{\prime \prime}, S_{\mathrm{A}}\right)$ shown in Fig. 3.7(a). Even though this grammar already reflects the role of the components of the assembly it is of marginal generality. It does, for instance, not describe assemblies where $B O L T_{1}$ would be replaced by a bolt called $B O L T_{1234}$. However, the name of an object should be irrelevant for a structural description, it would rather suffice to know what kind of objects are assembled. This problem is treated in the next step.

3. Extrapolation of relations among object types: Project the set $T_{\mathrm{A}}$ of terminal symbols which denote individual objects onto a set

$$
T_{\mathrm{A}}^{\prime}=\left\{a \mid a_{i} \in T_{\mathrm{A}}, i \in \mathbb{N}\right\}
$$

of terminals denoting object types. This and a corresponding adjustment of the productions results in the grammar $G_{\mathrm{A}}^{\prime \prime \prime}=\left(N_{\mathrm{A}}^{\prime \prime}, T_{\mathrm{A}}^{\prime}, P_{\mathrm{A}}^{\prime \prime \prime}, S_{\mathrm{A}}\right)$ depicted in Fig. 3.7(b).

\footnotetext{
${ }^{5}$ Choosing the power set is necessary because an assembly might have complex features composed of features from elementary objects so that new functions will emerge, cf. Appendix A.2.
} 


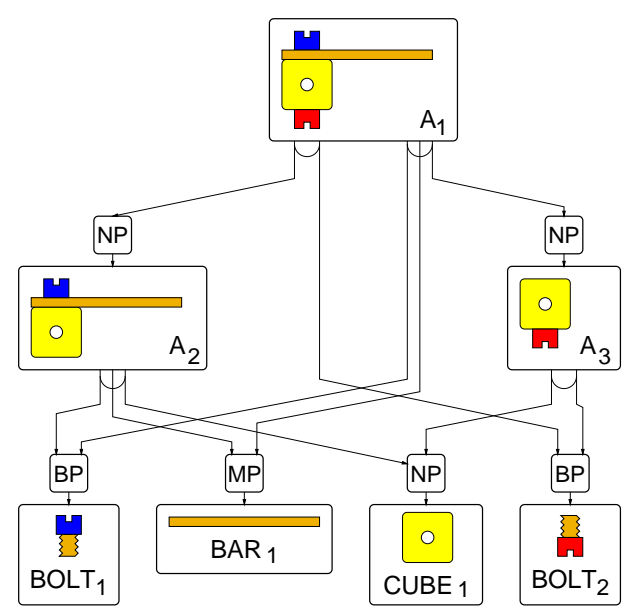

(a)

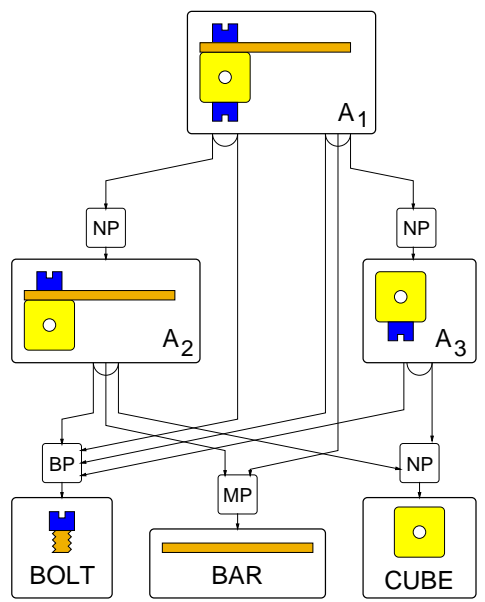

(b)

Figure 3.7.: 3.7(a) The AND/OR graph after the symbols $\mathrm{f}\left(\mathrm{A}_{2}\right), \ldots, \mathrm{f}\left(C U B E_{1}\right)$ have been renamed in order to indicate the functional significance of their succeeding nodes. 3.7(b) The graph after replacing symbols for individual objects by general object symbols and a corresponding unification of hyperlinks.

As the new grammar describes relations between object types instead of between individual objects, the visualization should not distinguish the two bolts anymore. Without loss of generality both bolts thus are drawn in blue.

4. Introduction of recursion: Finally, introduce a new variable $A$ and change the set of productions from $P_{\mathrm{A}}^{\prime \prime \prime}$ to $P_{\mathrm{A}}^{\prime \prime \prime \prime}$ where all variables $\mathrm{A}_{i}$ are replaced by $\mathrm{A}$ to annihilate the distinction between assemblies and subassemblies. If this results in productions deriving sequences of the same symbol $\mathrm{A} \rightarrow a b b \ldots b c$ where $b \in 2^{\Phi}$ and $a, c, \in 2^{\Phi *}$, introduce recursive productions that will allow to generate arbitrary sequences of symbols derived by $b$. I.e. substitute $\mathrm{A} \rightarrow a b c$ for $\mathrm{A} \rightarrow a b b \ldots b c$ and for each production $b \rightarrow d$ introduce an additional one $b \rightarrow d b^{6}$.

Finally, choose $S_{\mathrm{A}}^{\prime}=\mathrm{A}$ to be the new start symbol. The grammar $G_{\mathrm{A}}^{\prime \prime \prime \prime}=$ $\left(N_{\mathrm{A}}^{\prime \prime \prime}, T_{\mathrm{A}}^{\prime}, P_{\mathrm{A}}^{\prime \prime \prime \prime \prime}, S_{\mathrm{A}}^{\prime}\right)$ that results for our example is visualized in Fig. 3.8.

One might argue that the last step could have been carried out earlier and that is is unnecessary to introduce the functional recursion scheme for there could have been production like $\mathrm{A} \rightarrow \mathrm{A} \mid C U B E$. However, recursion by means of functional parts is more general and more efficient. Productions like the mentioned one would mean to enumerate

\footnotetext{
${ }^{6}$ Compare the productions MISC-PART $\rightarrow \ldots$ on page 31 .
} 


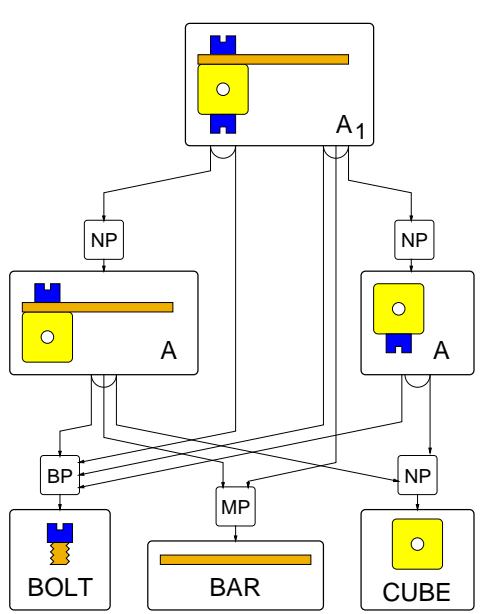

(a)

$\begin{aligned} \mathrm{A} & \rightarrow \mathrm{BP} \mathrm{NP} \mid \mathrm{BP} \mathrm{MP} \mathrm{NP} \\ \mathrm{BP} & \rightarrow B O L T \\ \mathrm{MP} & \rightarrow B A R \\ \mathrm{NP} & \rightarrow \mathrm{A} \mid C U B E\end{aligned}$

(b)

Figure 3.8.: The AND/OR graph and the corresponding grammar that result from substituting each variable $A_{n}$ by $A$ and choosing $A$ to be the new start symbol.

all decompositions of a certain example. And as we discussed in Chapter 2, the number of possible decompositions of more complex assemblies can become quite large.

In the case of our example this algorithm yields a grammar that generates the infinite set of words

$$
L\left(G_{\mathrm{A}}^{\prime \prime \prime \prime}\right)=\{B O L T, B O L T B A R\}^{+} \cdot\{C U B E\}
$$

that obviously includes sequences not corresponding to any feasible assembly. Like in the case we already discussed on page 32 , it contains, for instance, words composed of infinitively many bolts and a single cube which of course do not have physically feasible counterparts. In fact, it is impossible to rigidly attach more than four bolts to a baufix ${ }^{\circledR}$ cube thus each word of the language with more than four BOLT symbols does not represent a baufix ${ }^{\circledR}$ assembly. However, the language certainly represents every assembly that is composable from a single cube and an arbitrary number of bolts and bars. Therefore, we say that the exemplary assembly is a generator of a context free grammar that derives a language for baufix ${ }^{\circledR}$ assemblies containing bolts and bars and a single cube. This is the class of assemblies that contain a composite nut. Other exemplary assemblies will derive languages of assemblies containing complex bolts or complex misc parts. The resulting grammars can obviously be unified so that the general component structure of baufix ${ }^{\circledR}$ assemblies can be learned from examples. Some assemblies also may comprise all connection variants possible in the baufix ${ }^{\circledR}$ domain and thus enable to generate grammars for the class of all baufix ${ }^{\circledR}$ assemblies. In Fig. 3.1(b) we already saw an 
assembly that nearly meets the requirements for a generator of a comprehensive model. Except for a felly and a socket it contains all types of baufix ${ }^{\circledR}$ objects and every possible recursive relation between subassemblies can be observed.

Since the generalization of the algorithm is straightforward and it is not restricted to the treatment of baufix ${ }^{\circledR}$ assemblies, comprehensive assembly models for arbitrary domains can automatically be acquired from adequate examples. However, how to find sets of appropriate examples covering all structurally possible or relevant consists-of relations remains a problem for further research.

Now that we are acquainted with a simple generic mechanism for assembly modeling and its theoretical background we will discuss an approach to assembly detection in image signals. We will introduce several strategies to search for assembly structures by means of syntactic image analysis, discuss their advantages and drawbacks, and present characteristic results.

\subsection{Syntactic Approaches to Assembly Detection}

As we explained in the introduction, the cooperative construction scenario studied in the SFB 360 necessitates flexible assembly recognition from image data. We also discussed that this would best be based on generic representations of assembly knowledge and just saw that grammars provide an excellent means for this purpose. However, it remains to explore how they can be applied to assembly detection.

Analyzing complex configurations of elementary components with regard to a given grammar is called parsing. Parsing yields whether or not a complex structure belongs to a given formal language. Dealing with context free languages parsing is usually done by means of $\operatorname{LR}(k)$-parsers which analyze a pattern from left to right ${ }^{7}$. But assemblies are three-dimensional entities and images of assemblies are two-dimensional patterns. Therefore, even though assemblies correspond to words of context free languages, ordinary LR $(k)$-parsing cannot work and we need more sophisticated mechanisms for syntactic image analysis.

Following a hybrid approach that combines neural and semantic nets Kummert et al. [73] realized a reliable method to recognize elementary baufix ${ }^{\circledR}$ objects from vision. Their algorithm yields results in form of labeled image regions as shown in Fig. 3.9(b). Apparently, after object recognition assemblies appear as clusters of labeled regions which either are adjacent to or nested in each other. This of course is necessary for assemblies are 3D clusters of interconnected objects and photography is a linear, topology preserving projection form 3D to 2D. Mathematically, however, the presence of a region

\footnotetext{
${ }^{7} \mathrm{~A} \operatorname{LR}(k)$ parser reads a token of an input pattern and stores it on a stack. If there is a production $p_{i}=\left(n_{i}, \alpha_{i}\right)$ and $\alpha_{i}$ is on top of the stack, it will be replaced by $n_{i}$. This will be iterated until the whole input has been processed or an error occurred. As there may be several productions that would fit, the next $k$ tokens of the input are considered to constrain the choice of suitable productions [57].
} 


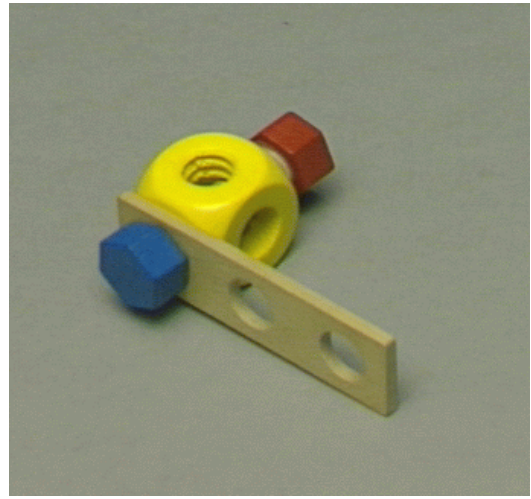

(a)

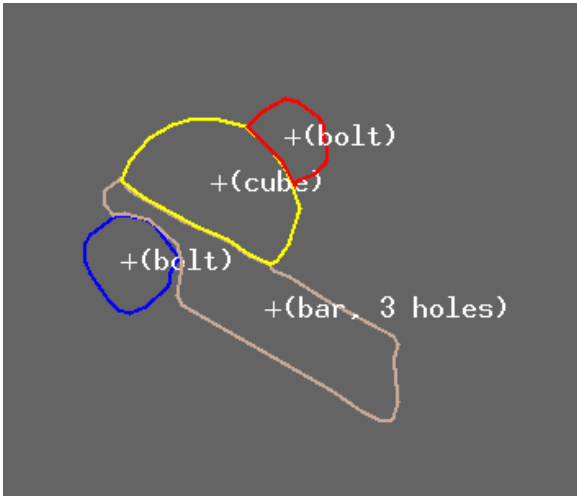

(b)

Figure 3.9.: The exemplary assembly known from Fig. 3.4(a) and corresponding results from a hybrid method for robust elementary object recognition introduced by Kummert et al. [73].

cluster does not provide a sufficient criterion for the presence of an assembly in an image because elementary objects may form clusters without being assembled. Camera perspective may even cause clusters to occur yet if there are only separated objects in the scene. But the existence of a region cluster is certainly a necessary precondition for finding assembly structures. Thus, we will assume $2 \mathrm{D}$ objects to be syntactic primitives and clusters of $2 \mathrm{D}$ objects to be the structures that must be parsed.

\subsubsection{Semantic Networks as Parsers}

In earlier work [12] we presented the idea to implement the recursive model of baufix ${ }^{\circledR}$ assemblies as a semantic network using ERNEST [71, 113].

ERNEST knows three different types of nodes and three different types of links. Concepts represent classes of objects, events, or abstract conceptions with certain general properties. They form the basis for semantical pattern analysis. Dealing with image understanding, for instance, interest lies in interpreting the sensor signal by means of given concepts. An instance is a copy of a concept where descriptions of common properties of a class are substituted by values derived from the signal. In an intermediate state of processing, instances may not be computable because certain prerequisites are missing. Nevertheless, the available information can be used to constrain an uninstantiated concept. This is done via the node type modified concept. Like in other semantic network formalisms part links decomposes a concept into its natural components. If a concept has several possible component structures, they can be modeled as different modalities. Another well-known kind of links are specializations. They realize an inheritance mechanism 
by which a concept inherits all properties of the general one. To distinguish knowledge from different levels of abstraction ERNEST introduces concrete links. Moreover, concepts are associated with attributes which represent numerical or symbolic features of the modeled term. Relations defining constraints for attributes can be specified and must be satisfied before valid instances can be generated. Since the results from initial segmentation seldom are perfect, concepts can also contain a judgment function to estimate the congruence between an image area and the term represented by the concept. The knowledge modeled in an ERNEST network is utilized in processes of creating and assessing modified concepts and instances. Assuming that complex object recognition means to detect all object parts, an object is considered to be recognized as soon as instances for all its parts were generated.

With all these features ERNEST is a powerful tool for pattern recognition. Within the scope of this thesis it is interesting to note that ERNEST networks with no other than part links are structurally equivalent to context free grammars ${ }^{8}$. Due to this equivalence the idea to implement generic assembly models as semantic networks is perfectly reasonable. It is in fact advantageous since semantic networks provide a way to deal with constraints among syntactic primitives that is missing in simple CFGs. Attributes assigned to concepts may represent knowledge or restrictions of non context free nature and thus provide opportunities known from attributed grammars (cf. [30]).

\section{Processing Model}

Our assembly model was mapped into an ERNEST knowledge base and attributes were defined which represent context sensitive and domain specific knowledge like, for example, the state of an object's mating features. This actually solves a problem mentioned earlier: the CFG that models baufix ${ }^{\circledR}$ assemblies allows to derive physically infeasible structures. The ERNEST implementation prevents this undesirable effect because attributes that register mating relations among objects suppress the generation of structures corresponding to infeasible attachments. Further attributes were introduced to support the control algorithm that governs the instantiation process. They register, for instance, which objects were already examined and estimate which objects should be considered next.

Given the semantic network as the processing model and the results from elementary object recognition as input data, detecting baufix ${ }^{\circledR}$ assemblies is a process of sequentially analyzing object clusters. Resulting hierarchical assembly structures are visualized as shown in Fig. 3.10(a). The figure shows a white polygon nested in black one, both surrounding images regions that were recognized to depict assemblies. The white one corresponds to a subassembly of the complex object enclosed by the black polygon; Figure. 3.10(b) depicts the corresponding syntactic structure.

\footnotetext{
${ }^{8}$ This is easy to see if we consider the AND/OR graph representation of a CFG: nodes correspond to concepts, AND links correspond to part links, and each hyperlink corresponds to a modality. A formal proof can be found in Appendix C.1.
} 


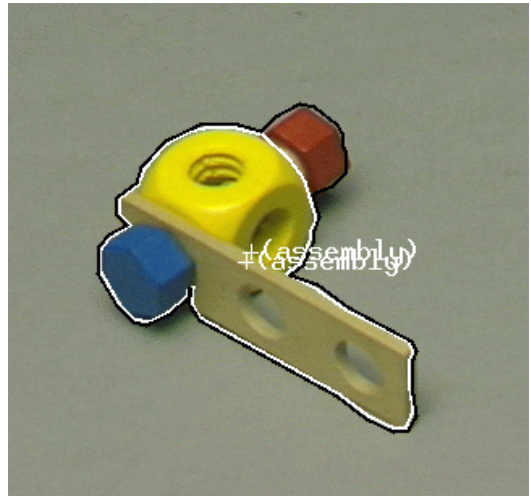

(a)

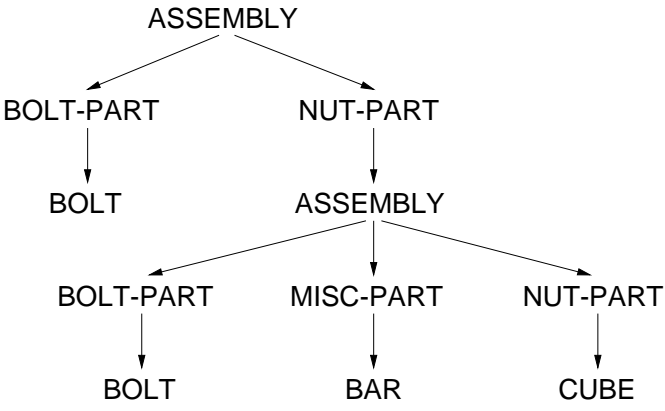

(b)

Figure 3.10.: Assembly detection result cast into the image and hierarchical structure corresponding to this result.

Details on the process of instantiating concepts from the knowledge base can be found in Appendix B.2. Here, we will only roughly sketch the underlying ideas: Since bolts are obligatory components of every baufix ${ }^{\circledR}$ assembly, analyzing a $2 \mathrm{D}$ cluster starts with a region representing a bolt. After instantiating the bolt as the bolt-part of an assembly, an attribute marks its image region as 'considered' and the adjacent objects are examined. If one of them complies with a criterion for choosing objects for the next step of the analysis and depicts a miscellaneous object the corresponding concept is instantiated and its region is marked 'considered' as well. This process iterates until a cube or a rhombnut are found such that an assembly can be instantiated. If an assembly was found but the cluster still contains unmarked objects, it is determined what role the assembly may play within a more complex unit, then it is instantiated as the corresponding functional part of another assembly, and finally the analysis is continued respectively.

In order to obtain a consistent syntactic interpretation for a $2 \mathrm{D}$ cluster its elements must be examined in a certain sequence. Generally, a method to order higherdimensionally distributed primitives for parsing is domain dependent and has to be found heuristically. In the baufix ${ }^{\circledR}$ domain, however, simple topological considerations avoid misinterpretations. Objects that are put onto bolts are linearly arranged in space, thus, as Fig. 3.11(a) underlines, the centers of mass of their image regions are ordered approximately linear as well. This defines directions in the image plane which, if estimated at the beginning of an analysis or after a (sub)assembly has been instantiated, can be used to search for further objects ${ }^{9}$. Hence, objects to be instantiated in a (sub)assembly

\footnotetext{
${ }^{9}$ The center of a bolt region and the center of an adjacent region, for example, define an initial search direction. If there are several neighbors, the control algorithm must consider alternative directions in a backtracking manner if one of them fails to yield a good interpretation.
} 


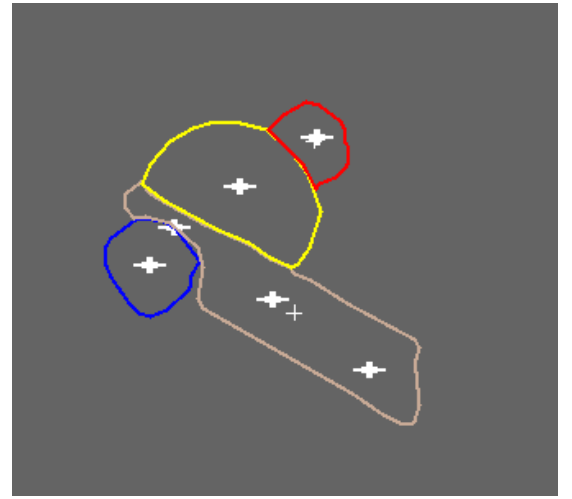

(a)

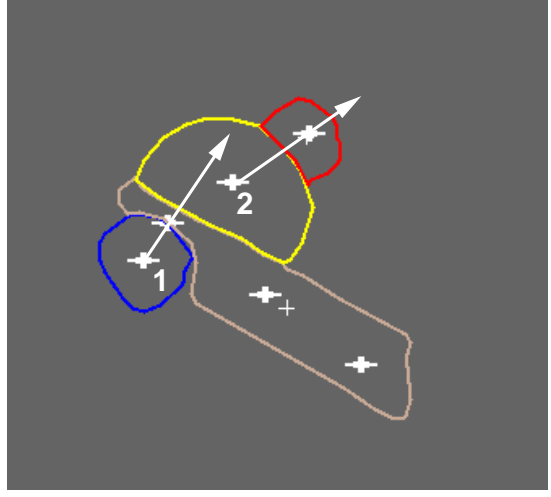

(b)

Figure 3.11.: 3.11(a) Positions of the centers of mass of the bolts and the cube and calculated positions of holes of the bar. 3.11(b) The directions for sequential cluster analysis yielding the result in Fig. 3.10.

must be adjacent to each other and if there are several neighbors, one of them should comply with the current search direction. Objects deviating from these directions are not assumed to belong to the current (sub)assembly. If an adjacent object depicts a bar the positions of its holes have to be considered instead of its center point and since holes used in a connection are not visible, their positions are calculated by dividing the bar's main axis into equidistant ranges.

Arrow 1 in Fig. 3.11(b) shows the initial search direction for our example. It is given by the center of the blue bolt's head and the position of the nearest hole of the bar. Arrow 2 in Fig. 3.11(b) indicates the direction for searching after a subassembly has been found that serves as a nut. It is defined by the center of a cube that was assumed to be attached to another bolt and a corresponding reference point of an unmarked neighboring object. Further details on this search heuristic can be found in [9] and [13].

\subsubsection{Assembly Parsing and Discourse Theory}

As the research conducted within the SFB 360 concerns human perception and communication and their emulation on intelligent machines, efforts are not only spent on aspects of computer vision but also on automatic speech recognition, man-machine dialog, and discourse parsing. Furthermore, the scenario necessitates the integrated treatment of both modalities and thus enforces intense cooperation among researchers from both fields. It were these fortunate circumstances that revealed a similarity between discourse and assembly structures and inspired another parsing strategy. But before we describe how 


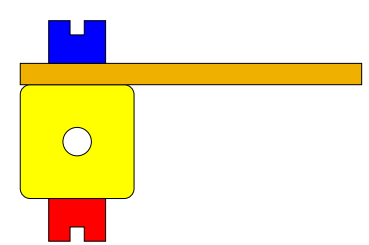

(a) Assembly $A_{1}$

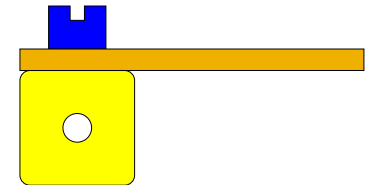

(b) Subassembly $A_{2}$

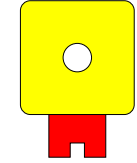

(c) Subassembly $A_{3}$

Figure 3.12.: The already known assembly $A_{1}$ and its two complex subassemblies $A_{2}$ and $A_{3}$. Both subassemblies contain the yellow cube. Therefore, they are said to be conjoined in $\mathrm{A}_{1}$.

principles of discourse processing can be transformed into strategies for visual assembly detection, we shall first familiarize us with general facts about discourse analysis.

Intuitively, discourse grammars specify how to combine structural primitives resulting from an investigation of discourse structure and discourse relations [114]. It is generally agreed that discourse has recursive structure, i.e. in discourse smaller segments with common reference points are recursively related [44]. Therefore, a discourse grammar consists of a sentence grammar, usually chosen to be unification based, and a set of discourse productions which describe discourse relations. This defines a framework in which intra- and intersentential constraints can be expressed because it integrates discourse constraints as well as syntactic and semantic restrictions. Moreover, often the processed discourse structure is only partially available for further processing. This means that only subparts with special properties are labeled as admissible parts for subsequent interpretation [134]. Summarizing, discourse grammars generate structures based on semantic and syntactic knowledge which capture various discourse phenomena [67].

In order to transform discourse theory into a framework that describes the internal structure of assemblies the structural properties of assemblies must match those of discourse. That is, assemblies must have a recursive structure where complex units can be obtained from recursively embedding less complex ones. Furthermore, there must be a relation between subassemblies that allows to unify their structures into larger units. As outlined above the consists-of relation naturally imposes recursive structures on mechanical assemblies. A relation between subassemblies was not introduced yet, but there actually is one. If two subassemblies share elementary objects, they can be conjoined to yield larger units. Figure 3.12 exemplifies this by means of the already familiar assembly $A_{1}$. Since its complex subassemblies $A_{2}$ and $A_{3}$ both contain the yellow cube, they are conjoined in $\mathrm{A}_{1}$. 


\section{Processing Model}

The structural similarities between discourse and assemblies inspired a new parsing strategy for assembly detection in image signals [11]. It adapts an approach to discourse parsing due to Kronenberg [68, 69] who proposed to apply a LR(1)-parser together with a unification grammar. The advantage of her approach is that the unification grammar puts most of the syntactic information that is standardly captured in context free phrase structure rules into a lexicon. Every word in the lexicon is represented by a feature structure which specifies the values of various attributes. This resembles the definition of concepts and attributes of elementary objects in the ERNEST network for assembly detection. Thus, the part of our knowledge base that describes elementary assembly components can be identified with a lexicon for discourse parsing.

A LR(1)-parser standardly carries out two actions called shift and reduce. The reduce action reduces the right hand symbols of a grammatical production to the left hand symbol. Kronenberg augmented a standard LR(1)-parser with the facility to handle feature structures. As a consequence, every reduce action includes a unification of discourse feature structures yielding structural descriptions of larger elements. This, in turn, corresponds to the instantiation of ASSEMBLY concepts. Instantiating a concept after all its parts have been instantiated can be interpreted as a reduce action. Moreover, within the ERNEST formalism instantiating a concept requires to (re)calculate its attributes. Since we defined attributes that register the states of all mating features comprised in an assembly, the instantiation of an assembly thus also includes a unification of feature structures. The shift action pushes symbols from and input string onto a processing stack. In the semantic network this can be simulated by the attributes that select the objects for examination and realize sequential cluster analysis as describe above.

These observations motivated a modified approach to assembly detection. Its basic idea is trying to find small units in an object cluster (similar to discourse segments in speech parsing) and to conjoin them with earlier found ones, if possible. Thus, assembly structures (or derivation trees) resulting from the analysis of a subpart of a cluster are little by little conjoined with already derived structures. Subcluster parsing terminates if the cluster is completely analyzed or if the unification fails. The latter occurs if two objects should be instantiated as parts of an assembly but the feature structure of one of them does not allow further unification. If, for example, all holes of cube were already associated with bolts, the cube must not be related with another bolt.

Merging of subassemblies is done by a conjoin operation that manipulates derivation trees (also denoted assembly trees). It integrates already derived trees (which are called current assembly trees according to discourse theory terminology) into incoming assembly trees (which is another name for the recently derived assembly tree).

Definition: The conjoin operator $\triangle$ combines two assembly trees $X$ and $Y$ if both share an elementary object. In $\mathrm{X} \triangle \mathrm{Y}$ node $n$ of $\mathrm{X}$ is substituted by $\mathrm{Y}$, if and only if $n$ 


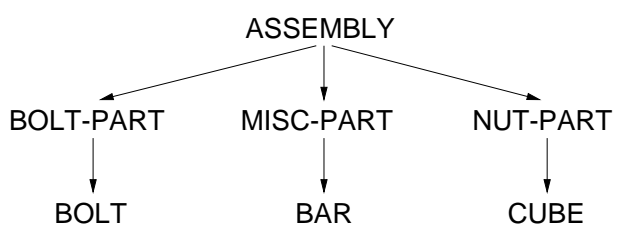

(a)

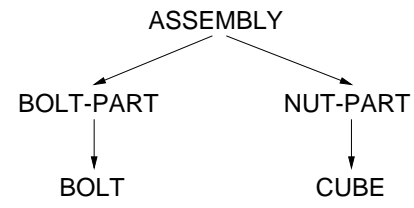

(b)

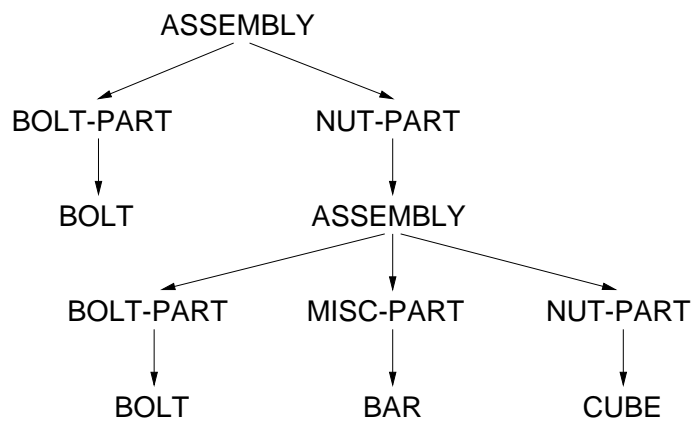

(c)

Figure 3.13.: 3.13(a) Current assembly tree describing subassembly $A_{2}$ of assembly $A_{1}$. 3.13(b) Incoming assembly tree describing subassembly $A_{3}$ of assembly $A_{1}$. 3.13(c) Assembly tree representing $A_{1}$ as $A_{3} \triangle A_{2}$.

represents a common object (see Fig. 3.13 for an example). If $X$ and $Y$ do not share an object, $\mathrm{X} \triangle \mathrm{Y}$ is undefined.

Thus, each time an assembly was instantiated, the modified algorithm for cluster analysis tests if other assemblies have been instantiated so far which share an elementary object with the recent one; if so, a conjoin operation is performed. Three different cases can occur:

1. No conjoinable current assembly tree is found. This either happens if no other subassembly was detected so far or if there only are substructures which do not share an object with the incoming one. If there are further unexamined objects in the cluster, the analysis will continue. To this end, it is determined what role the recently detected assembly might play in a more complex structure and the algorithm tries to instantiate corresponding instances. Since the decision concerning the functional role is based on local evidence, i.e. guessed from adjacent cluster objects, it might be wrong. If the global structure of the cluster does not support it, the analysis attempt will fail. In that case the analysis will restart at another part of the cluster. If after instantiating an assembly there are no further unexamined objects, cluster analysis has been completed and the process terminates.

2. One or several of the current assembly trees and the incoming one can be conjoined. These trees will be integrated into the incoming one and the analysis continues as sketched above.

3. Several common elementary objects are found in a current assembly tree and several corresponding nodes in the incoming assembly tree must be substituted. This 


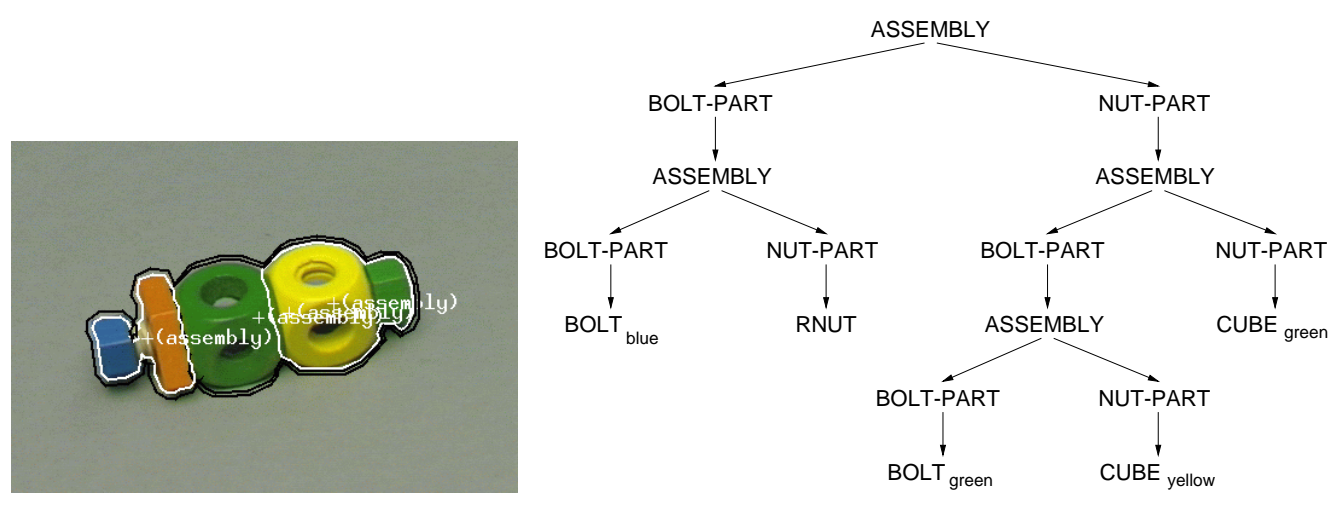

(a)

(b)

Figure 3.14.: Image of an assembly with detection result cast into it and the corresponding syntactic structure. Structures like this cannot be found with the original version of the assembly detection algorithm.

case occurs if assemblies are connected via several elementary objects; an example is presented in Appendix B.1.

The major advantage of the modified algorithm is its ability to detect nonlinear assembly structures. The original approach to cluster analysis employs a straight bottomup instantiation strategy: if a structure is found in a cluster, its possible role will be estimated from the adjacent objects, i.e. from its context in the cluster, and the analysis will continue correspondingly. If there are several alternatives but all corresponding instantiation attempts fail, the analysis will stop. This approach can only yield linear structures as shown in Fig. 3.3 and Fig. 3.10(b). The notion of linearity was introduced by Wolter [135] and characterizes assemblies which can be constructed by adding elementary objects to complex units. They can be represented by syntactic structures where each hierarchical level contains only a single complex component. Most baufix ${ }^{\circledR}$ assemblies will be linear assemblies, however, there also are nonlinear ones.

Figure 3.14 shows an object that cannot be assembled linearly but rather requires to connect subassemblies that were constructed separately. The detection result cast into the image was obtained from the parsing strategy based on conjoining subassemblies. The corresponding syntactic structure is shown on the right. Obviously, it is not linear; the highest hierarchical level contains two complex parts. Structures like this cannot be detected in a pure bottom-up instantiation process. 


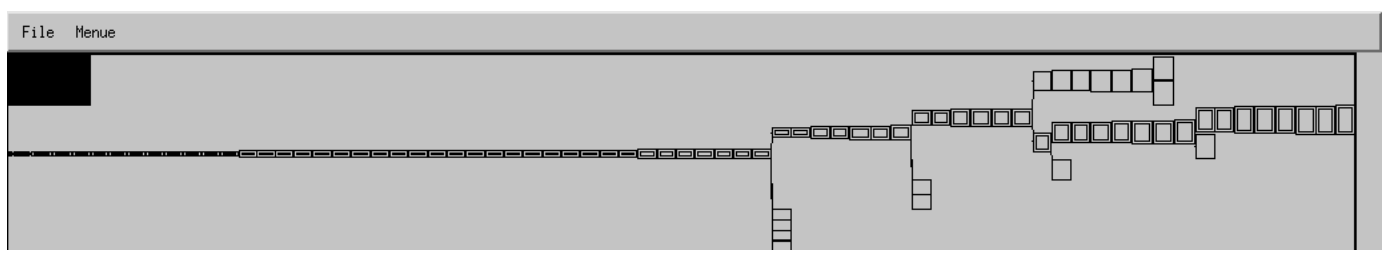

Figure 3.15.: ERNEST search tree that was generated while analyzing the assembly in Fig. 3.14. The double framed boxes represent search tree nodes that lead to valid instances while the single framed nodes represent instantiation attempts that failed or alternatives that were not considered any further.

\section{Implementational Issues}

Realizing the detection algorithm that adopts ideas from discourse parsing required a slight modification of the original knowledge base. An additional concept ASSEMBLYANCHOR was introduced to keep track of possible starting points for cluster analysis.

Figure 3.15 visualizes the instantiation process that yielded the result for the nonlinear assembly we just discussed. The figure shows the search tree generated by ERNEST while processing the corresponding object cluster. Each assembly detection process starts with reading and clustering elementary objects and with generating corresponding instances. Here, this preliminary processing leads to the line of search tree nodes on the left. After all elementary objects were instantiated the tree branches for the first time. There are five possibilities to start assembly detection since an attribute of the ASSEMBLYANCHOR concept yielded the two bolts and the two cubes as alternatives starting points. It also produced a fifth branch that may be use as a fallback state for the analysis if the other four alternatives would fail.

Choosing the green bolt to start the analysis results in the instantiation of an assembly composed of the green bolt and the yellow cube. Since the cluster contains further unexamined objects, it has to be estimated what function this assembly might have and as there are several alternatives the search tree branches again. The first branch appears because green bolts allow more objects than a single cube to be attached to them. Thus, the assembly might act as a BOLT-PART. Since the yellow cube is adjacent to an unexamined object the assembly might also be the NUT-PART of a more complex object. The third branch represents another fallback state. Pursuing the BOLT-PART assumption yields an assembly of a complex bolt and the green cube and again possible functions must be be estimated. This time a BOLT-PART assumption is impossible because green bolts with two cubes cannot be attached to further objects. But since the green cube is adjacent to an unexamined object, the assembly might be a NUT-PART. The second branch at this point of the tree yet again represents a fallback.

Pursuing the NUT-PART hypothesis does not lead to a result. The underlying syntactic model requires that after a NUT-PART has been instantiated as the first component of an 
ASSEMBLY some miscellaneous parts or a bolt will follow. Here, however, there is another nut adjacent to the green cube. Thus, the last fallback must be taken into account and another instance of ASSEMBLY-ANCHOR is generated in the corresponding search tree node. This time, only the blue bolt remains as a possible starting point for subcluster analysis because the other objects of the original set of candidates are already integrated into structural descriptions. The blue bolt and an obligatory fallback thus yield two alternatives for further processing. The first alternative yields an assembly consisting of the bolt and the rhomb-nut that may serve as a BOLT-PART. Pursuing this assumption yields that the cube adjacent to the rhomb-nut is part of a current assembly which therefore is conjoined with the incoming one. Afterwards, there are no objects left to be examined and the analysis terminates.

\subsection{Spinoffs from Syntactic Assembly Detection}

So far, we have seen that context free grammars are capable to describe the composition of mechanical assemblies and that grammatical parsing is suited to detect assemblies in image data. Assembly detection is accomplished by means of semantic networks and extends ordinary parsing because in addition to syntactic structures semantic networks can also recognize feature structures. Next, we will see that this reveals mating relations among parts and thus leads to topological unique descriptions of assemblies. Furthermore, we will discuss how the syntactic model of baufix ${ }^{\circledR}$ assemblies enables to detect mating operations in an assembly environment and that integrating results from action and assembly detection can improve the respective results. Finally, we will explain how knowledge encode in our grammatical model may also support the recognition of elementary objects.

\subsubsection{Learning Assembly Sequence Plans from Vision}

On pages 43 and 44 we discussed the need for sequencing $2 \mathrm{D}$ clusters of recognized baufix ${ }^{\circledR}$ objects to enable syntactic parsing. Sequencing was accomplished using a heuristic drawn from topological properties of baufix ${ }^{\circledR}$ assemblies. In this section, we will explain that sequential cluster analysis also may be used to derive assembly plans from computer vision. Next, however, we shall consider the formal background of our heuristic; this will require the following definitions:

A set $A$ is said to be partially ordered by a relation $\sqsubseteq$ (usually pronounced weaker than) if the following properties hold for every $x, y, z \in A$ :

1. $x \sqsubseteq x$.

2. $x \sqsubseteq y$ and $y \sqsubseteq x$ implies $x=y$.

3. $x \sqsubseteq y$ and $y \sqsubseteq z$ implies $x \sqsubseteq z$. 


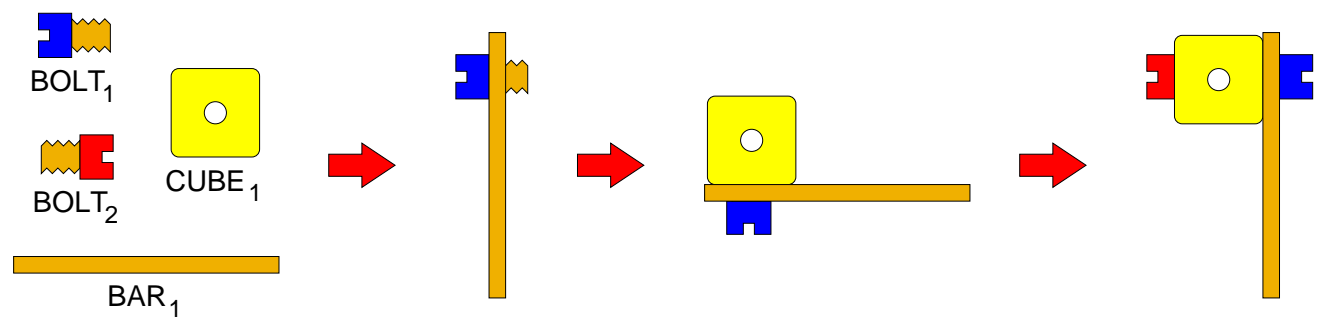

Figure 3.16.: A sequence to assemble the familiar assembly $\mathrm{A}_{1}$.

Let $A$ be a partially ordered set. An element $a \in A$ is a maximal element if there is no $b \in A, b \neq a$ such that $a \sqsubseteq b$. An element $a \in A$ is called a minimal element if for no $b \in A, b \neq a, b \sqsubseteq a$. $A$ is called a chain if for all $a, b \in A, a \sqsubseteq b$ or $b \sqsubseteq a$. Note that every subset of a chain is a chain. Given a partially ordered set $A$ and a chain $C \subseteq A$, we say that $C$ is a maximal chain in $A$ if

$$
\nexists C^{\prime} \subseteq A: C \subset C^{\prime}
$$

I.e. a chain is maximal if it is not a subset of any other chain.

Now that we know about partially ordered sets and chains consider the following

Definition: Let $o_{1}$ and $o_{2}$ be two elementary objects such that either one of them is a bolt $B O L T_{i}$ and the other is attached to it, or none of them is a bolt but both are connected to $B O L T_{i} . o_{1}$ carries $o_{2}$ on $B O L T_{i}$ if one of the following conditions holds:

1. $o_{1}=o_{2}$, or

2. $o_{1}$ is $B O L T_{i}$ and $o_{2}$ is attached to it, or

3. $o_{1}$ was attached to $B O L T_{i}$ ere $o_{2}$ was attached to it.

If $o_{1}$ carries $o_{2}$ on $B O L T_{i}$ we will henceforth write $o_{1} \sqsubseteq c_{i} o_{2} \cdot{ }^{10}$

From this definition we see that each bolt in a bolted assembly generates a relation that imposes a partial order on the set of compound objects. Moreover, such orders will always be chains. ${ }^{11}$ However, the name of the relation seems a little odd. We will motivate it by means of the familiar assembly $A_{1}$. Suppose $A_{1}$ is assembled as shown in Fig. 3.16. First, $B A R_{1}$ is attached to $B O L T_{1}$. If oriented as sketched in the figure, $B O L T_{1}$ will carry $B A R_{1}$ and we may write $B O L T_{1} \sqsubseteq_{c_{1}} B A R_{1}$. After attaching $C U B E_{1}$ to the bolt and in a corresponding orientation, $B O L T_{1}$ and $B A R_{1}$ will both carry $C U B E_{1}$. Thus, will also have $B O L T_{1} \sqsubseteq_{c_{1}} C U B E_{1}$ and $B A R_{1} \sqsubseteq_{c_{1}} C U B E_{1}$. The dependency on the orientation is of course undesirable. Therefore, the relation $x$ carries $y$ on $z$ should be understood abstractly. It is meant to express that, with respect to $z, x$ precedes $y$ or $x$

\footnotetext{
${ }^{10}$ Appendix C.2 motivates why the relation is chosen with respect to a bolt.

${ }^{11}$ See Appendix C.2 for a proof.
} 


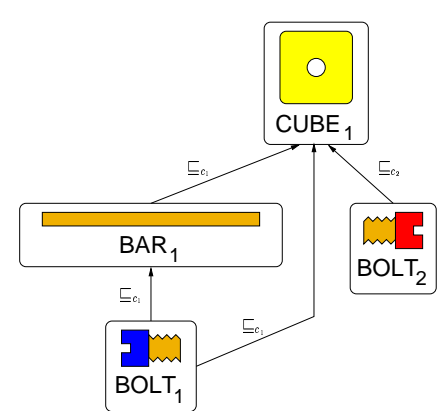

(a)

\author{
$B O L T_{1} \sqsubseteq_{c_{1}} B A R_{1} \sqsubseteq_{c_{1}} C U B E_{1}$ \\ $B O L T_{2} \sqsubseteq_{c_{2}} C U B E_{1}$
}

Figure 3.17.: A graphical representation of the ordering relations in $A_{1}$ and the two maximal chains contained in the assembly.

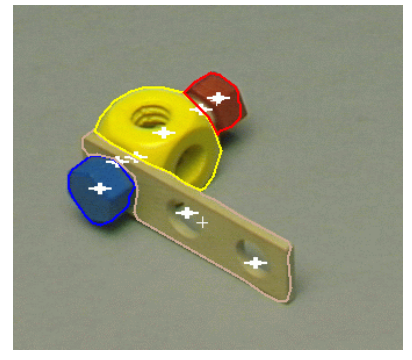

(a)

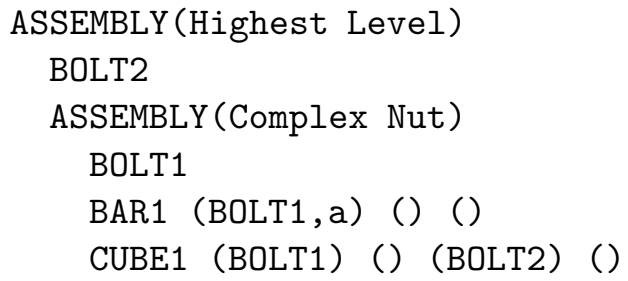

(b)

Figure 3.18.: Calculated positions of mating features and a high-level assembly sequence plan for the assembly $A_{1}$.

is below $y$. Finally, if $B O L T_{2}$ is attached to the assembly, it may be seen as a carrier of the cube as well and we will have $B O L T_{2} \sqsubseteq_{c_{2}} C U B E_{1}$. But as it neither is in direct contact with $B A R_{1}$ nor with $B O L T_{1}$, it should not be interpreted to carry those.

The ordering relations within assembly $A_{1}$ can be graphically summarized as shown in Fig. 3.17(a). We easily recognize two maximal chains; Fig. 3.17(b) shows them using the notation we just introduced.

Since bolted assemblies necessarily consist of at least two objects, each chain in an assembly has a minimal element $b$ and a maximal element $n$. Object $b$ will always be a bolt and $n$ will be a nut. Thus, as $b \neq n$, these elements can be thought to define a line.

Hence, lines defined by sequentially arranged objects are a general topological property of bolted assemblies. The basic idea behind our heuristic was that similar structures must be found in images of baufix ${ }^{\circledR}$ assemblies since photography preserves topology. And indeed, there are approximately linear arrangements: the center points of image regions associated to bolted objects define lines in the image plane along which syntactic analysis 


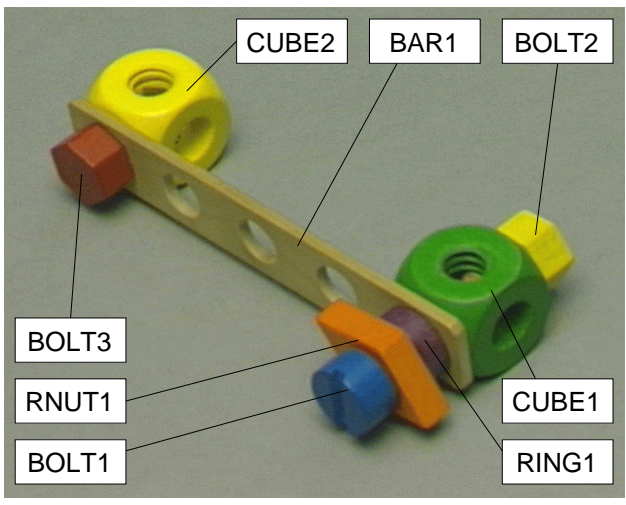

(a)

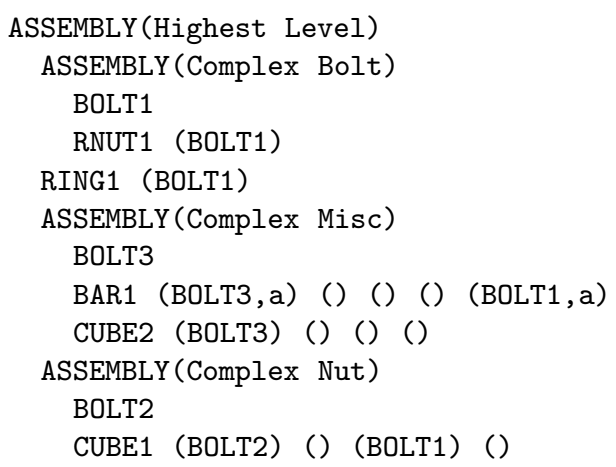

(b)

Figure 3.19.: The assembly known from Fig. 3.1(b) and a possible sequence plan. The syntactic component structure of this plan corresponds to the detection result discussed in Appendix B.

can be performed. For objects like rhomb-nuts or rings these center points are assumed to represent the projected position of the corresponding mating feature. For bars this assumption is certainly invalid. However, their mating features are assumed to be equally spaced along the principle axis of the corresponding image region. And in fact, this yields positions which comply with the postulated linear arrangements. Dealing with cubes the center point will likewise not represent a mating feature. Thus, mating features of cubes are assumed to be found at the points of intersection between the estimated lines and the border of a cube's region (see Fig. 3.18(a)).

Given their image coordinates, it is possible to estimate which mating features participate in a connection. Object attributes defined in the ERNEST network for assembly detection can verify whether a feature belongs to one of the lines that are due to a partial order. If so, the feature is assumed to be attached to the corresponding bolt. The detection algorithm can therefore generate descriptions of assemblies which describe hierarchical component structures and simultaneously reflect relations among mating features. Data structures that include both kinds of information correspond to what Chakrabarty and Wolter [24] call high-level assembly sequence plans. Examples of sequence plans for baufix ${ }^{\circledR}$ assemblies generated from visual inspection are shown in Figs. 3.18 and 3.19.

The plan in Fig. 3.19 results from the detection process discussed in Appendix B. Its overall structure reflects, that certain complex parts of the assembly can be constructed in parallel (cf. Fig. 3.1(a)). Objects with holes occur with (lists of) slots representing their mating features. Although cubes have six surfaces, in the plan they only have four slots since baufix ${ }^{\circledR}$ cubes feature only four threaded holes. If a feature is occupied, it contains the name of the corresponding bolt. Slots of bars are additionally marked 
with a flag that indicates whether the corresponding bolt is inserted from above (a) or below (b). These flags are defined intrinsically: each instance of a bar has an attribute to register the temporal order in which the bar's mating features were considered during cluster analysis. The bolt in the first of these holes is assumed to be above the bar. Other bolts associated with that bar are above as well if they are on the same side of its principle axis. Those on the other side are said to be below. The order of the slots of bars and cubes is also defined intrinsically. Holes of a cube, for example, are listed in a clockwise manner: the hole that was considered first during analysis will always appear as the leftmost slot, the one intrinsically left to it is represented by the second slot, its vis-a-vis corresponds to the third slot, and the hole on its right appears last in the list. Further details on this labeling scheme can be found in [13].

Plans like this characterize how the elementary assembly objects are connected to each other. Syntactic component structures augmented with information of mating feature relations thus yield topologically unique descriptions of an assembly ${ }^{12}$. High-level plans derived from vision therefore may be translated into appropriate natural language or manipulator instructions in subsequent steps of processing.

\subsubsection{Combining Assembly and Action Detection}

In order to find correct assembly structures from vision all elementary parts of an assembly must be visible and recognizable. Thus, if syntactic assembly detection should yield accurate results the analyzed object clusters must be complete and correct, i.e. for each elementary object of an assembly there has to be a corresponding region which is labeled correctly. However, taking images of three-dimensional objects often implies perspective occlusions which may cause some of their parts to be just partially visible or even hidden. And since partially visible objects might be misclassified, neither the prerequisite of correctness nor that of completeness of a cluster can be guaranteed. Experience shows that for the baufix ${ }^{\circledR}$ domain this is a rather relevant problem if an assembly contains small objects. These are often missing in its image so that syntactic analysis will produce incomplete structures. In $[8,9]$ we proposed a solution to this problem which we will roughly sketch in the following.

While the previous sections discussed assembly detection from a single image, this section will describe how processing image sequences may yield assembly structures. The basic idea is to monitor construction processes, to detect mating operations, and to deduce assembly structures therefrom. We will see that fusing the results from both approaches can cope with slight occlusions and thus increases robustness and reliability in visual assembly detection.

As the cameras in our experimental setting provide a global view of the assembly cell and there are no dedicated devices to monitor the manipulators or the hands of

\footnotetext{
${ }^{12}$ Geometrically, however, they are still ambiguous since they do not regard rotations about bolts.
} 
Pick $X$

Preconditions: hand: empty $\wedge X$ on table $\wedge X \rightarrow$ disappears

Effects: $\quad$ hand: $X \wedge \neg(X$ on table $)$

Connect $X Y$

Preconditions: hand ${ }_{1}: X($ BOLT-PART $) \wedge$

hand $_{2}: Y($ MISC-PART $) \vee($ NUT-PART $)$

Effects: $\quad$ hand $_{1}: X Y \wedge$ hand $_{2}$ : empty

Place $X$

Preconditions: hand: $X \wedge X \rightarrow$ appears

Effects: $\quad$ hand: empty $\wedge X$ on table

Figure 3.20.: A simple set of rules to infer assembly actions from the observation of appearing or disappearing objects in the assembly cell. The rules assume two handed assembly and the notation hand ${ }_{1}$ and hand $\mathrm{d}_{2}$ points out that there are two distinguished hands but does not denote a specific hand or manipulator.

the constructor, mating operations cannot be observed directly. The approach to action detection therefore has to be indirect and simple. It reuses results from the object recognition module and infers assembly actions from symbolic information: analyzing if and which objects appeared in or disappeared from the scene enables to deduce what has been constructed. The major advantage of this indirect approach is its ability to detect operations independent of whether they are carried out by a human or a robot.

The basic assumption behind all inferences made by our algorithm is that complex objects are constructed from a sequence of two-handed assembly tasks. Two handed assembly characterizes a mode of construction where there are two hands or manipulators and each of which can handle one part at a time. According to this assumption, two hands are modeled which may hold at most a single object or a single (partial) assembly. Given these models, a collection of rules is used to hypothesize actions from changing contents of the scene. On this level of abstraction, rules to infer Pick, Place, and Connect actions can be defined which are shown in Fig. 3.20.

Adopting the notation from planning literature [92], each rule itemizes a couple of conditions or events and an assembly operation that can be concluded therefrom. If the scene changes and the requirements of a certain rule are met, the corresponding action is hypothesized and the models are updated accordingly. While Pick and Place actions are directly observable, Connect operations can only be deduced from temporal context: if two objects have been taken, there is no hand left to take another one. Thus if another objects disappears the ones in the hands must have been connected (which of course requires that they provide appropriate mating features) so that a hand was free for 

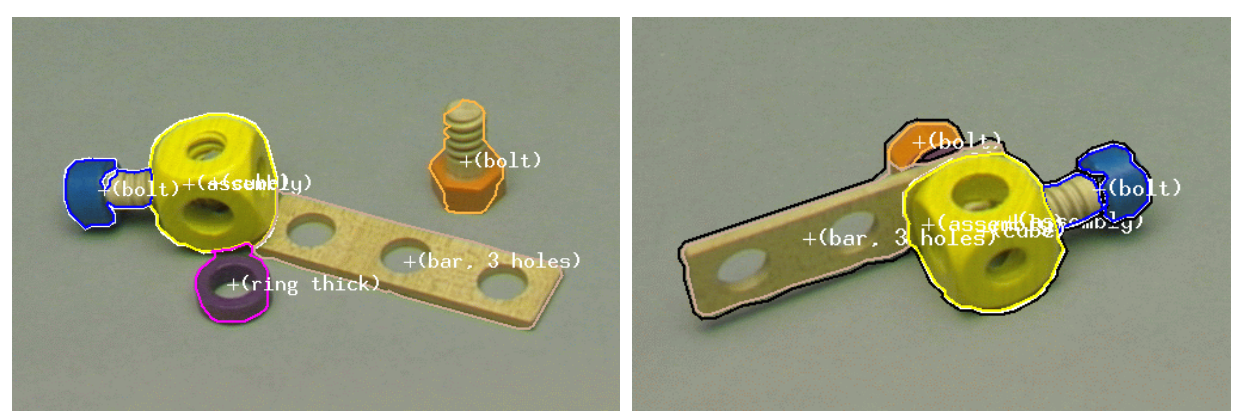

Figure 3.21.: Images depicting different stages of a construction process. Note that due to perspective occlusion the purple ring was not recognized in the right image. This caused the assembly detection procedure to yield an erroneous structure.

taking. A minor drawback of indirect inferences like this is that they require a certain level of cooperation from subjects acting in the scene. The following restrictions should be regarded during construction in order to avoid confusion:

- Each hand must only hold a single object or a single (partial) assembly.

- Objects must only be put down within the field of view of the system.

- New objects must only be introduced to the scene while no identical objects are in the hands of the constructor.

Generally, after a sequence of assembly tasks was detected, one of the hand models will contain a set of connected objects. But from Fig. 3.20 we see that Connect operations require one of the hands to hold a BOLT-PART. Observing a series of Pick and Connect operations thus means to observe sequential attachments of parts to a bolt. Therefore, the set of interconnected objects must be partially ordered. And since its structure was derived from symbolic information that usually does not suffer from perspective occlusion, it provides a reliable description of the assembly whose construction was observed. However, details of mating relations cannot be extracted from symbolically detected Pick and Connect events.

As mentioned above, syntactic assembly detection yields connection details, but perspective occlusion may cause structures to be incomplete. Fusing structures from action and assembly detection can cope with this problem and may provide better results than obtainable from the individual algorithms. In order to integrate both approaches we consider the partially ordered sets they yield. For example, after the assembly depicted on the right of Fig. 3.21 was put into the scene, the purple ring was not recognized and syntactic cluster analysis resulted in:

$$
B O L T_{\mathrm{b}} \sqsubseteq_{c_{\mathrm{b}}} C U B E \text { and } B O L T_{\mathrm{o}} \sqsubseteq_{c_{\mathrm{o}}} B A R \sqsubseteq_{c_{\mathrm{o}}} C U B E
$$


whereas analyzing its construction process yielded:

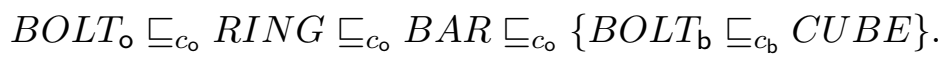

where $\sqsubseteq_{c_{\mathrm{b}}}$ and $\sqsubseteq_{c_{\mathrm{o}}}$ denote the ordering relations due to the blue and the orange bolt, respectively. Note that action detection yielded relations between elementary objects and a set of objects which represents the assembly depicted on the left of Fig. 3.21. This in fact is reasonable because the assembly was taken during the construction process and thus has to appear in one of the hands. It is obvious from this example that simple set comparisons will indicate whether tiny objects are missing in the result from visual assembly detection but are contained in the structure generated by action detection. They thus can easily be inserted into the syntactic structure.

\subsubsection{Application of Syntactic Context}

So far, we have learned how the semantic network that implements the context free grammar of baufix ${ }^{\circledR}$ assemblies detects composite objects from image data. Next, we will see that knowledge of the component structure of assemblies encoded in the network offers further opportunities for computer vision: contextual constraints implicit to the syntactic assembly model and information gathered during cluster analysis may support elementary object recognition.

Within the SFB 360 considerable efforts are spent in different approaches to recognition from vision. We already mentioned a hybrid mechanism that combines neural and semantic networks and processes characteristic features of homogeneous image regions in order to recognize elementary baufix ${ }^{\circledR}$ objects [73]. For the same purpose, contour based techniques were developed, too, as were ideas for integrating different vision paradigms into a single system (cf. [115] and [18], respectively).

All these joint efforts led to a robust and reliable computer vision system that was reported in [112]. A detail of its architectural layout is sketched in Fig. 3.22. It shows the central module for elementary object recognition which integrates different cues provided from lower level image processing. But there also are two feedback loops with the higher level assembly detection component.

The interaction between these modules is twofold because on the one hand, syntactic assembly detection may provide additional cues for the classification of certain image regions. On the other hand, it can rate competing classifications and thus support the decision for the most likely one. We shall explain both mechanisms by means of examples.

\section{Generating Cues for Elementary Object Recognition}

Contextual knowledge gathered while parsing a cluster of objects allows to generate hypotheses for unrecognized image regions. Figure 3.23 exemplifies this inference mechanism. The leftmost image shows an assembly and the results from region based object 


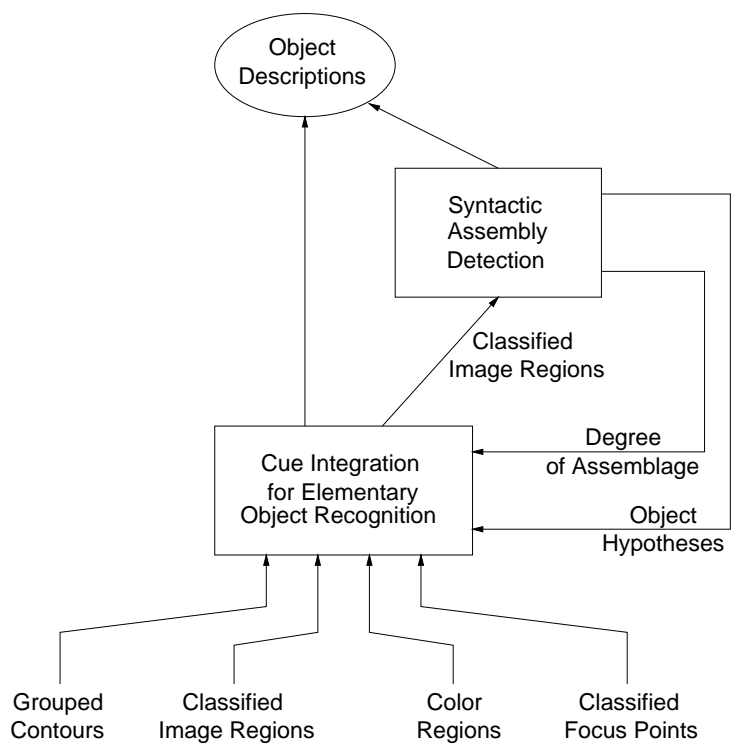

Figure 3.22.: Detail of a computer vision architecture that combines several processing units into a robust and reliable recognition system for baufix ${ }^{\circledR}$ objects.

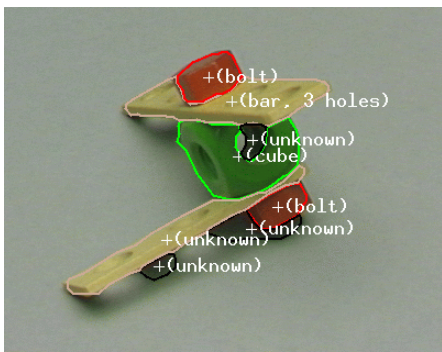

(a)

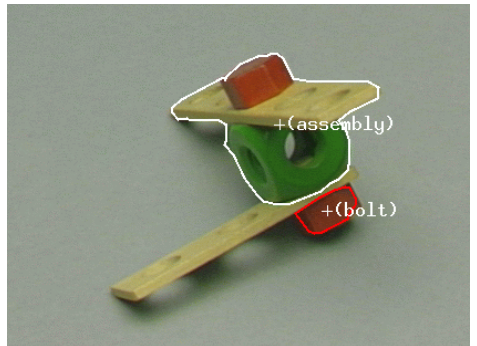

(b)

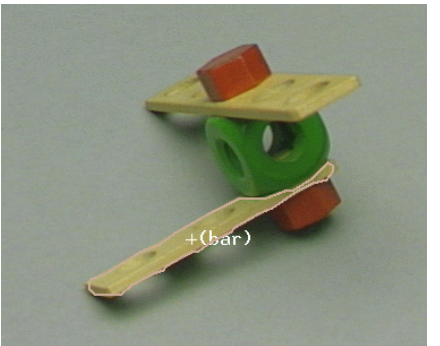

(c)

Figure 3.23.: 3.23(a) Assembly and results from elementary object recognition where the lower bar could not be recognized. 3.23(b) Results from syntactic assembly detection. 3.23(c) Hypothesis generated during the parsing process.

recognition. Note that there are some shadowy regions which were not classifiable and thus are labeled unknown. Apart from these, there is another unknown region which in fact represents a bar but could not be recognized as such.

The second image shows the assembly detection result. Syntactic cluster analysis started at the upper bolt, continued with the adjacent bar and then found the cube. Consequently, an ASSEMBLY was instantiated. Cluster parsing restarted at the lower bolt which was instantiated as the BOLT-PART of another assembly. At this stage of the 


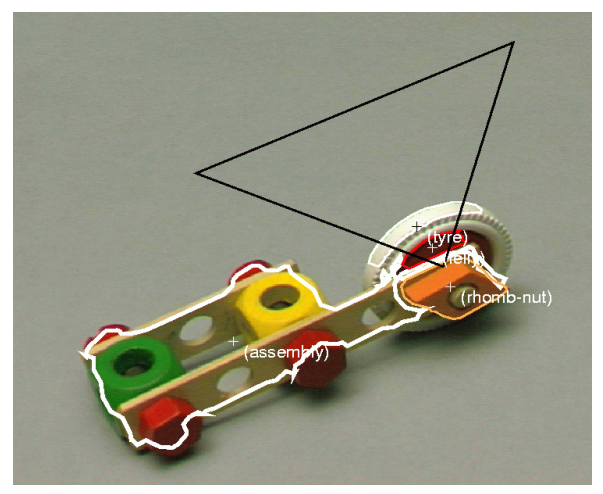

Figure 3.24.: Assembly with detection result and angular range where the parser would expect a bolt from contextual conditions.

analysis, there were two regions directly adjacent to the bolt which left two alternatives to continue parsing. But since one of the regions is represented by a black polygon, it was correctly assumed to depict shadow and was hence neglected. The remaining region, however, does not have an object label either and could thus not be instantiated as an assembly component.

Nevertheless, in situations like this, labels for unknown cluster elements can be guessed from the current state of the analysis. It just has to be considered what kind of part was instantiated last and which parts would accord with the syntactic assembly model if they were instantiated next. If, like in the example, a BOLT-PART was instantiated last, one would expect to find a miscellaneous object or a nut subsequently rather than another bolt. Such expectations can be generated for any partial assembly structure, i.e. for any case where some but not all parts of an assembly are instantiated. Therefore, it is generally possible to restrict the type of unknown cluster objects and to provide plausible cues for elementary object recognition.

In the example the unknown region is of wooden color and has a certain extension. This narrowed the choice of possible labels even further and the region was hypothesized to represent a bar (see Fig. 3.23(c)). However, hypotheses are not integrated into structural descriptions for they may still be wrong. Instead, they are transferred back to the module for elementary object recognition that, in the next processing cycle, integrates them with cues from lower level modules.

Inference from syntactical context not only allows to generate hypotheses for unknown cluster objects but also enables to predict objects which cannot be seen at all. Figure 3.24 shows an assembly we already know from the introduction. Here, the image also contains a polygon surrounding the area corresponding to the most comprehensive assembly structure that could be found. Note that the rhomb-nut, the felly, and the tyre are not part of this area. They were not instantiated as assembly components because 
Object

Recognition:

Assembly
Detection:
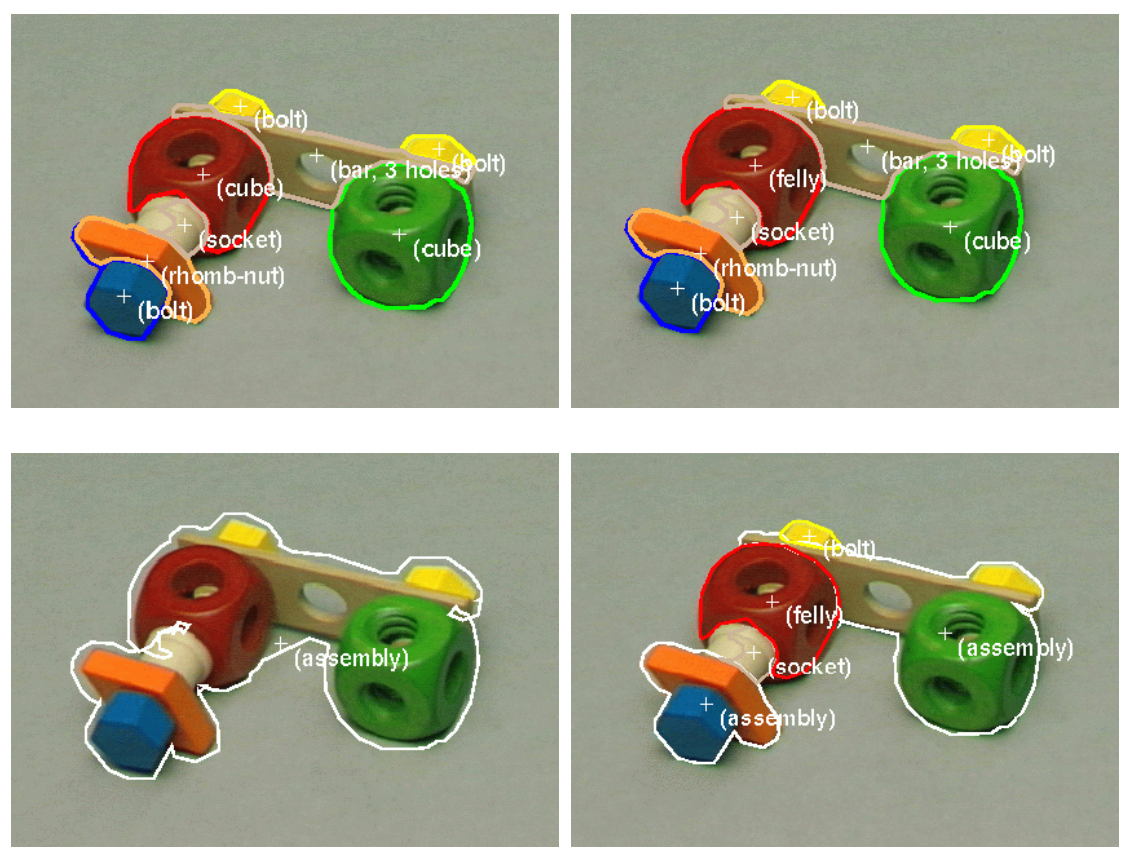

Figure 3.25.: Two competing recognition results (in the alternative on the right, the red cube was wrongly classified as a felly). Assembly detection yielded a degree of assemblage of 1.0 for the left and of 0.3125 for the right alternative.

the bolt they are attached to is not visible. However, during the analysis the rhomb-nut was chosen as a starting point for parsing ${ }^{13}$. In that parsing attempt the linear arrangement of the nut, the adjacent bar, the felly, and the tyre hinted that these elements are very likely to form an assembly if there only was a bolt. The black triangle indicates the admissible angular range in the image where the semantic net would expect and accept the bolt.

Expectations like this can actually be used to coordinate perceptions in cooperative man-machine interaction. If the assembly detection module is integrated into a system for multi-modal cooperation in assembly (cf. [7]) and has to process a scene like the exemplified one, it may verbalize its expectations. Or, it may request the user to slightly move the assembly in question so that a complete structure might be generated.

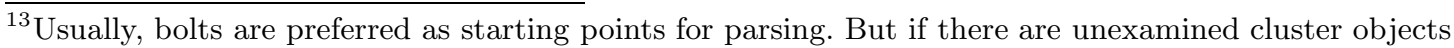
and none of them is a bolt, other obligatory parts are chosen instead. As a nut the rhomb-nut did meet this criterion.
} 


\section{Assessing Competing Object Recognition Results}

Figure. 3.25 exemplifies the second type of interaction between elementary object recognition and assembly detection. The upper row shows two competing object recognition results which are due to inconsistent cues from lower level vision. On the right, the red cube was wrongly classified as a felly, a mistake which occurs frequently for features of cubes and fellies computed from region segmented images are in fact very similar. However, if there are competing interpretations of regions contained in a cluster, syntactic assembly detection can provide evidence which alternative might be correct.

As we see in the lower row of Fig. 3.25, assembly detection yielded a structure including all cluster elements for the alternative on the left. Whereas the recognition error in the right alternative caused the detection of two disjoint assemblies and furthermore prevented some objects of the cluster to be associated with an assembly. Under the assumption that a cluster of labeled regions very likely depicts a single assembly, phenomena like this indicate that there are misclassified regions.

However, a cluster must not depict an assembly at all. Hence, a binary decision between right or wrong should be avoided, instead, a measure of the likeliness of a labeling scheme would be preferable. Therefore, we introduc the degree of assemblage of an object cluster.

Definition: Let $C$ be a cluster of objects and let $|C|$ be the number of its components. Let $a(C) \leq|C|$ denote the number of objects in $C$ which are part of an assembly and let $A(C)$ be the number of disjoint assemblies found in $C$. Then the degree of assemblage $\alpha(C)$ of an object cluster $C$ is given by the quotient:

$$
\alpha(C)=\frac{a(C) /|C|}{A(C)}
$$

Thus, $\alpha$ sets the number of disjoint assemblies detected in a cluster against the number of objects found to be part of an assembly and the total number of cluster elements. If there are few distinct assemblies and the ratio of assembled objects and cluster elements is high, i.e. $a(C) \approx|C|$, then $\alpha$ will be high and the input data for assembly detection is likely to be correct. If the number of disjoint assemblies found in a cluster is high and only few of its objects are assembled, i.e. $a(C) \ll|C|$, then the degree of assemblage will be low and the results from elementary object recognition seem not to be reliable. In our example, $\left|C_{l}\right|=\left|C_{r}\right|=8$ for both alternatives. For the (correct) left alternative we have $a\left(C_{l}\right)=8$ and $A\left(C_{l}\right)=1$ while assembly detection based on the (faulty) right result yields $a\left(C_{r}\right)=5$ and $A\left(C_{r}\right)=2$. Thus $\alpha\left(C_{l}\right)=\frac{8 / 8}{1}=1$ and $\alpha\left(C_{r}\right)=\frac{5 / 8}{2}=\frac{5}{16}$ which hints that the result depicted on the upper left of Fig. 3.25 should be preferred.

Especially in cases of severe perspective occlusion, the recognition of elementary object gains from this scheme of module interaction. On a test set of 26 images of assemblies where many components were just partially visible the recognition accuracy 
could be improved by $10 \%$ [112]. As we will see in Chapter 6 , this of course affects the performance in assembly detection. Right now, we can conclude that the integration of lower level image processing and higher level analysis of symbolic information suggests an avenue towards more robust and reliable recognition in computer vision.

\subsection{Syntactic Methods in Another Domain}

In Chapter 3.1.2 we figured out that context free grammars can describe the component structure of mechanical assemblies. This result was synthesized from work by Homem de Mello and Sanderson and a contribution by Hall; the former introduced AND/OR graphs as a standard method to represent all consists-of relations within an assembly and the latter noted the equivalence of AND/OR graphs and context free grammars some 30 years ago. Section 3.1.2 also presented a method to learn grammatical models from examples. The resulting CFGs do not simply enumerate individual assembly structures but constitute general models of a whole class of assemblies. This was accomplished from considerations of the functional role of subassemblies in mechanical connections. Understanding assemblies as compositions of functional units led to general recursive productions and therefore to compact and comprehensive models.

Sections 3.2.1 and 3.2.2 presented the idea to implement the CFG of baufix ${ }^{\circledR}$ assemblies as a semantic network using ERNEST . Given the grammatical primitives as labeled image regions, we also introduced suitable control strategies which enable the detection of baufix ${ }^{\circledR}$ assemblies in image data. Hence, ERNEST networks can principally act as grammatical parsers on higher-dimensional input.

Next, we will exemplify that our method developed for modeling mechanical assemblies is not restricted to domains of physically connected objects: compact context free grammatical models with recursion over functional components can be found for any set of interrelated objects. This is rather obvious for any non trivial set contains elements and subsets, i.e. it consists-of of smaller units. Thus, any entity that results from relating two or more less complex ones consists-of several parts and can be structured according to AND/OR graphs or CFGs, respectively. (It may, however, be tricky and a question of experience to identify such entities and their components in an arbitrary domain.) Furthermore, we shall corroborate the suitability of semantic networks as grammatical parsers in structural pattern recognition. To this end we will briefly discuss a domain of more abstractly related objects and introduce a suitable and comprehensive context free model together with a corresponding ERNEST implementation that parses yet another kind of higher-dimensional data.

\subsubsection{Syntactic Models to Structure Room Scenes}

This section presents preliminary work on syntactical environment modeling which is part of initial efforts in a research project on service robotics. The project investigates 


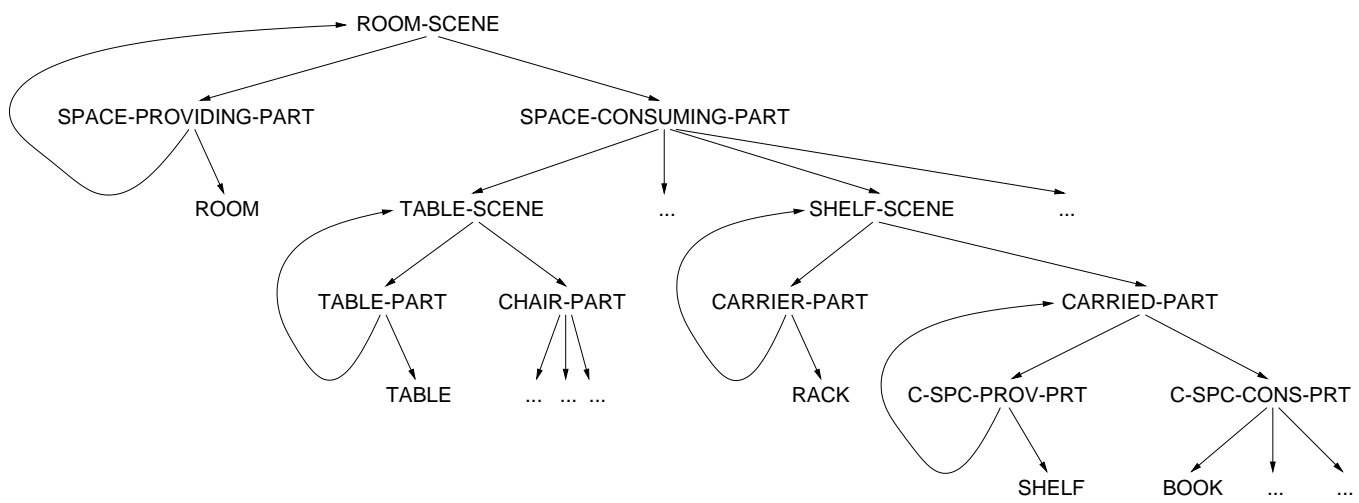

Figure 3.26.: Syntactic model of a room scene. A room scene is decomposed into two functional units, the one that consumes space might be represented by several subscenes which again can have recursive component structures.

how to instruct and steer a mobile robot in ordinary home or office environments. It does not primarily aim at traditional problems of mobile robotics like autonomous exploration or path planning but, like the SFB 360, is focused on man-machine interaction. And similar to the cooperative construction scenario, the intended service robot should rely on visual and acoustic input while communicating with a user. Capabilities like selflocalization or obstacle avoidance, in contrast, may also rest on laser or sonar sensors but need not to be restricted to these.

A mobile robot that has to navigate in an environment of several distinct rooms certainly needs a mechanism to estimate its current position. Consequently, there is intensive research on problems of global and local localization and literature on these issues is vast (cf. [43] for an overview). Besides approaches applying sensors like sonar or laser rangefinders, computer vision based techniques are very popular. These usually make use of landmarks which may be objects characteristic for a location or visual markers attached to, for example, walls. As the latter constitute an artificial and auxiliary means generally not present in ordinary environments, they are ineligible for our setting. Thus, questions of self-localization from vision motivated preparatory work on 3D furniture recognition [50]. However, some pieces of furniture might be present in several rooms and thus are of no use for localization. Others might be characteristic but unrecognizable due to perspective occlusion. This led to the idea to take contextual information into account and to model the furnishing of entire rooms syntactically.

Figure 3.26 illustrates how this might be done applying the techniques presented so far. It depicts a general syntactic model of the component structure of a room scene. The model assumes that a ROOM-SCENE is made of two functional parts: one that provides space and one that consumes space. The space providing unit might either be an empty room or a room with some furniture in it, i.e. a ROOM-SCENE. Typical groups of furniture 
are space consuming units. A group of chairs arranged around a table, for instance, can be recursively decomposed into a chair and a table with a lesser number of chairs. Thus, a TABLE-SCENE could be composed of a TABLE-PART and a CHAIR-PART. The TABLE-PART might be a TABLE or a less complex TABLE-SCENE while the CHAIR-PART could be one of several elementary types of chairs.

Other space consuming parts may have more complex structure. A SHELF-SCENE, for instance, should generally consist of a unit that carries several shelves. As the number of shelves is arbitrary this CARRIER-PART is either a RACK or a SHELF-SCENE itself. And as the shelves carried by a rack may contain several books or other items each CARRIED-PART of a SHELF-SCENE is again recursively structured.

In contrast to mechanical assemblies, this syntactic model of a ROOM-SCENE and its components is not bound to functions of mating features. The functional relations between the variables are rather abstract instead and the model was deduced from experience. Nevertheless, if there was a set of suitable relational representations of room scenes the algorithm presented in Section 3.1.2 would be able to produce a similar context free grammatical model.

While the concept of mechanical mating features thus seems superfluous in deriving context free models of classes of complex entities, the next section will show that it still is advantageous when implementing detectors for syntactical structures.

\subsubsection{An Experimental Implementation}

Intending to verify the applicability of syntactic methods in detecting constellations of furniture from sets of 3D object data, we slightly modified the TABLE-SCENE part of the room model and implemented it as an ERNEST network. The modification further generalized the model in order to allow the treatment of scenes like shown in Fig. 3.27(a) which contain more than one table. Assuming binary partitions of complex scenes resulted in the following CFG:

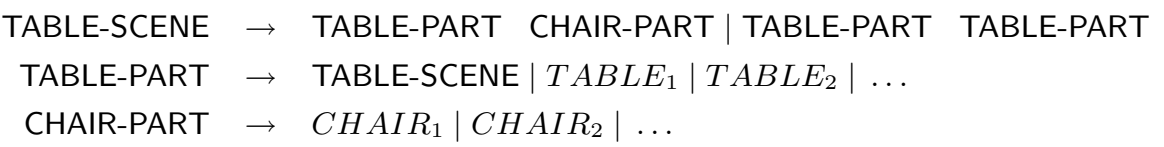

A TABLE-SCENE may thus consist of a TABLE-PART and a CHAIR-PART or of two TABLEPARTs. And while the CHAIR-PART cannot be of complex nature, a TABLE-PART may have recursive structure.

Implementing the grammar as an ERNEST knowledge base is straightforward. Variables and terminals are mapped to concepts and productions are represented by suitable part links. However, as mentioned above, the data that should be searched for syntactic 


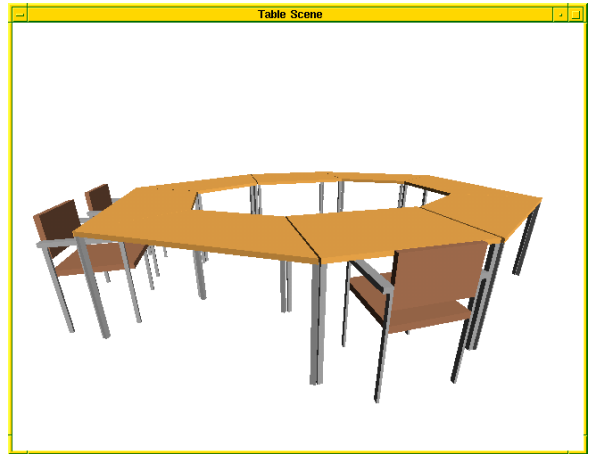

(a)

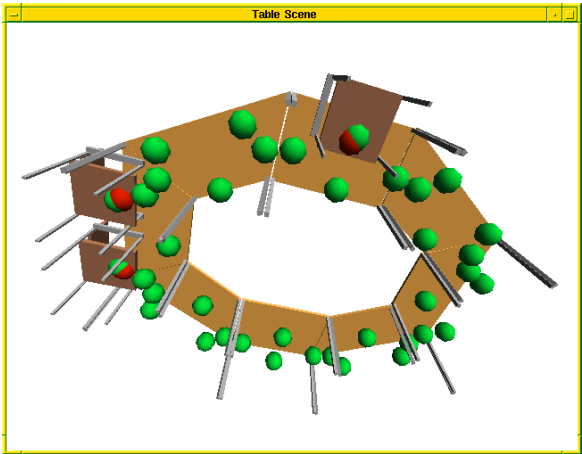

(b)

Figure 3.27.: A table scene consisting of several chairs and tables and corresponding positions of virtual mating features. Green balls associated with tables indicate positions near which one would expect a chair or another table to be situated; red balls indicate the parts of chairs that usually are near a table.

structures results from 3D object recognition, i.e. suitable strategies to parse sets of 3D object models have to be found.

A practicable control algorithm to detect table scenes can actually be adopted from the ideas developed for the baufix ${ }^{\circledR}$ domain. On the first sight, this might surprise since the ERNEST network for baufix ${ }^{\circledR}$ assembly detection continuously makes use of the idea and properties of mating features. Remember that their properties not only led to the syntactic model of bolted assemblies. Considerations regarding their appearance and distribution in an image guide the detection of assembly structures and allow to deduce connection details. But there are no such entities in the furniture domain. Instead, pieces of furniture might form a group without being physically connected.

However, though they are more abstract and not as obvious than in the case of mechanical assemblies, relations among pieces of furniture can yet be stated geometrically. A chair, for instance, has an intrinsic front which in a common table scene is oriented towards a table. Thus, although there are no rigid connections there still are geometrical or spatial constraints that apply to well formed table scenes. Such constraints, of course, can easily be modeled by means of attributes or restrictions associated with concepts of an ERNEST network.

But spatial relations among three-dimensional objects are inherently fuzzy. For example, a chair near a table might be oriented towards the table. Likewise, there will be orientations where the chair does not point to the table. But then there also will be orientations where it somehow does and somehow does not point towards the table. In 


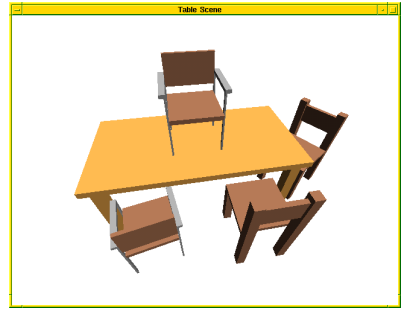

(a)

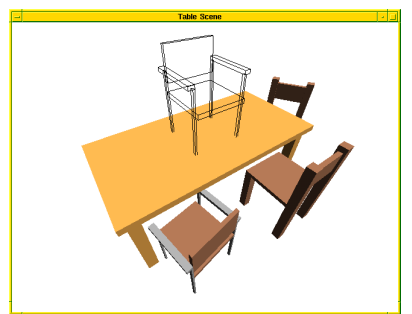

(b)

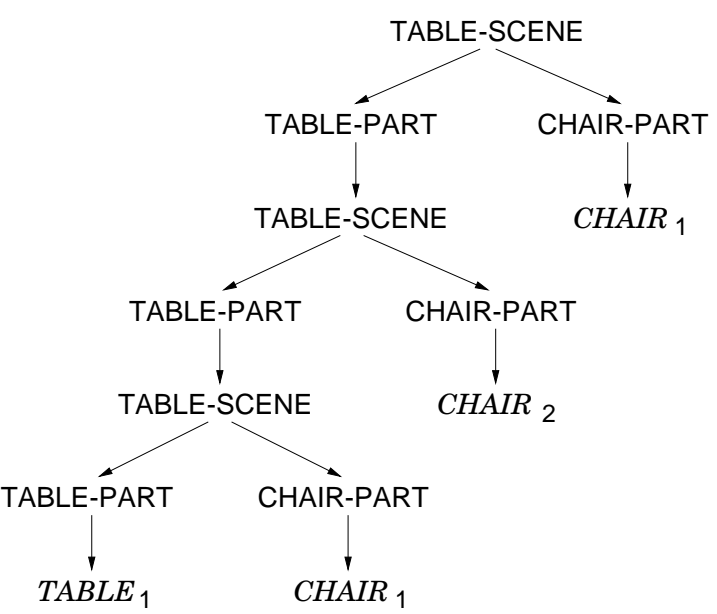

(c)

Figure 3.28.: 3.28(a) A scene of several pieces of furniture. 3.28(b) The table scene detected within this configuration. 3.28(c) Syntactic structure resulting from a parsing process.

other words, there are fuzzy transitions from one orientation to the other where a binary decision will be difficult.

Actually, this phenomenon can be observed for any relation expressed with spatial prepositions and there is intensive research on automatic spatial reasoning and semantic modeling of spatial prepositions (cf. e.g. [1, 42, 94]). For our purposes we were not interested in especially sophisticated computational models but looked for an easy and practicable way to calculate spatial restrictions for well formed table scenes. In analogy to mechanical assemblies and in order to facilitate computations and to adopt our established control strategy we thus assigned virtual mating features to the geometric furniture models. Figure 3.27(b) shows such features assigned to exemplary pieces of furniture. Each table is associated with a set of 3D points which are indicated by green balls. They represent prototypical positions round the table near whom one would usually expect to find a chair or another table. Chairs come along with points indicated by red balls which represent the part of a chair that, in a common table scene, is most likely to be found near a table.

The idea of virtual mating features apparently simplifies the search for interrelated objects in a set of furniture models. Constraints for well-formedness could be stated using any measure of distance between virtual features. In the current implementation we simply apply the Euclidean distance in $\mathbb{R}^{3}$ but more sophisticated methods, as for 
instance proposed in [1], would be possible, too. And like in the case of baufix ${ }^{\circledR}$ assemblies positions of mating features can guide the parsing process performed by the semantic network.

Again the ERNEST knowledge base contains attributes which represent context dependent, domain specific knowledge like, for example, the states of virtual mating features. And in analogy to the baufix ${ }^{\circledR}$ domain, there are attributes which govern the instantiation process for they register examined objects or estimate how to continue parsing.

Given sets of 3D object models as input data, the semantic network searches them for table scenes similar to the method introduced in Section 3.2.2. A table is an obligatory part of any table scene. Thus, after instantiating concepts for all objects in the input data, a table serves as the starting point of a first parsing attempt. It is instantiated as a TABLE-PART which in turn is annotated as a part of a modified concept of a TABLE-SCENE. Then, all other objects with unused virtual mating feature in a certain range about an unused feature of the initial table are considered as possible further components of the TABLE-SCENE. The nearest one that complies with the syntactic model is instantiated correspondingly, e.g. as a CHAIR-PART, and the TABLE-SCENE becomes and instance as well. The features responsible for this instantiation are henceforth considered to be used and the TABLE-SCENE is conjoined with earlier found ones, if necessary. Afterwards, parsing restarts at another table if there is any or continues with unexamined objects near to already instantiated ones. This process iterates until all objects in the input data have been examined. Figure 3.28(b) exemplifies how detected structures are visualized. It depicts a result obtained from the scene in Fig. 3.28(a). In this example, the chair upon the table is not close enough to any of the table's virtual features to be understood as part of a conventional table scene. Consequently, it was not instantiated as such and thus appears as a wireframe model in the output.

The syntactic structure which the semantic network produced for this table scene is shown in Fig. 3.28(c). Since there is just one table to initialize parsing, no conjoin operations were necessary and the resulting structure is linear (see page 48). Two of the three chairs included in the structure are of type $C H A I R_{1}$. The one at the right front of the table is closest to a virtual mating feature of the table and thus is represented on the lowest level of the parsing tree. The $\mathrm{CHAIR}_{2}$ type chair at the left front is to be found on the next level and, even though the perspective is confusing, the chair in the back is farthest from the table and thus integrated on the highest hierarchical level.

\subsection{Summary}

Syntactic approaches to pattern recognition are known for long (cf. e.g. [20, 40]). Benefiting from the powerful mechanism of recursion grammatical models usually provide compact representations of patterns of interrelated primitives. And given a correspond- 
ing parser, formal grammars allow to classify complex patterns and simultaneously yield structural descriptions.

Grammatical formalisms have been introduced to computer aided manufacturing as well. They were used for workpiece or machine tool design [19, 95] or for modeling in assembly sequence planning [121]. But syntax-based contributions to reasoning in assembly make use of rather complex formalisms like shape or graph grammars and simple grammatical approaches were not yet reported. Moreover, to the best of our knowledge they have not yet been applied to assembly recognition.

This chapter dealt with mechanical assemblies from a pattern recognition point of view. Faced with the requirement of visual assembly detection in a dynamic and unpredictable environment we introduced a simple but flexible method of syntactic assembly modeling. We demonstrated that concepts from the early days of formal language theory are sufficient to model the component structure of individual mechanical assemblies as well as of whole classes of composite objects. The fundamental idea was to bring together concepts first introduced by Homem de Mello and Sanderson [53, 54, 55] and a discovery by Hall [49].

Dealing with assembly sequence planning, Homem de Mello and Sanderson proposed the use of AND/OR graphs to represent all feasible decompositions of mechanical artifacts. Hall, however, pointed out that AND/OR graphs and context free grammars as introduced by Chomsky [27] are equivalent formalisms since nodes of an AND/OR graph can be mapped one to one onto variables and terminals of a CFG while hyperlinks can be identified with context free productions. This proves that it is possible to represent assembly structures using simple grammatical formalisms.

Furthermore, this chapter showed that consideration of the mechanical function of subassemblies lead to particular compact representations of assembly knowledge. Understanding composite objects to consist of functional units results in recursive models of entire classes of assemblies. These general models can derive structural descriptions of all feasible assemblies of a certain class and may actually be learned from examples.

However, context free grammatical models of composite objects are rather coarse and do not represent topological or geometric relations. They cannot model even simple mechanical restrictions and thus may derive structures which are mechanically infeasible. Thus, more sophisticated syntax-based techniques like attributed grammars [30], graph grammars [87], coordinate grammars [84], or plex grammars [35] seem more appropriate since they could cope with context sensitive and geometrical constraints. But we pointed out that CFGs are structurally equivalent to a certain type of semantic networks. We demonstrated that CFGs can be implemented using the semantic network language ERNEST and that ERNEST knowledge bases can be employed in assembly detection from vision. By assigning attributes to ERNEST concepts domain dependent knowledge of non context free nature can be encoded. Thus, suitable attributes will prevent the generation of infeasible assembly structures from image analysis.

Discussions with researchers working on speech processing revealed a similarity be- 
tween discourse and assembly structures. And ideas originally developed for discourse parsing led to a robust algorithm for assembly detection from higher-dimensional data. The portability of concepts from discourse theory to assembly detection was corroborated by means of two exemplary implementations. One dealt with the originally considered cooperative construction scenario and the other transferred our methods to the detection of certain arrangements of pieces of furniture.

For the baufix ${ }^{\circledR}$ scenario we also presented a couple of extensions of our syntactical methodology. Observing that bolted assemblies can be understood as a collection of partial orders led to a heuristic that recognizes relations among mating features. Syntactic image analysis thus can also provide connection details so that there is topological information that allows to generate high-level assembly sequence plans. Moreover, in assembly detection from image data perspective occlusions are a rather frequent problem. They may cause erroneous structures or prevent assembly detection at all. We thus proposed to incorporate process knowledge in order to generate reliable assembly structures even if some elementary components were not correctly recognized. Finally, syntactic constraints inherent to our model can be used to support the recognition of elementary objects. To this end syntactic assembly detection is integrated into a feedback loop along with a module for elementary object recognition. Provided with incomplete data from the object recognition module the assembly detection unit hypothesizes labels for previously unrecognized image areas by regarding the syntactic context they appear in. Provided competing data from elementary object recognition assembly detection can rate the competitors by analyzing which of the alternatives yields the most likely assembly structures. 


\section{Mating Feature Graphs}

In the previous chapter, we saw that context free grammars provide a compact and flexible way to represent assembly knowledge. They can be implemented as semantic networks and instantiation strategies inspired from discourse parsing accomplish the detection of assembly structures in image data. However, the scenario of the SFB 360 necessitates more than just detection. The machine that assembles baufix ${ }^{\circledR}$ parts must be able to resolve references made by its instructor. For example, if the instructor wants a tailplane-fin to be manipulated, it is not enough to detect that there are several assemblies in the surroundings, the machine rather has to recognize which of them was meant. It must determine which of the detected structures corresponds to something known as tailplane-fin.

Certain hierarchical descriptions or assembly plans found from image parsing could of course be labeled and stored in a data base. Other plans yielded by scene analysis could then be matched against this set of prototypes in order to realize recognition on a syntactical level. Yet, in general, this will be error-prone and time-consuming. As we discussed in Chapter 3.1.2, mechanical assemblies might, on the one hand, have numerous syntactic structures. On the other hand, our parsing algorithm cannot be guaranteed to always yield the same structure of a complex assembly. Depending on its location and orientation in the image, object cluster parsing will start at different subparts of a cluster so that different syntactic structures are likely to result. We thus would need many representative descriptions for syntactic assembly recognition. However, the more complex an assembly is the more syntactic structures it will generally have. Experiments by Homem de Mello and Sanderson [55] and Wolter [136] even revealed the number of different structures to grow exponentially with the number of interconnected parts.

In this chapter, we shall first verify these observations for the assemblies of the baufix ${ }^{\circledR}$ domain. Then we will present an approach to assembly recognition that extends the syntactic methodology and allows to recognize assemblies without unreasonable efforts. We will introduce a graph representation of relations among mating features of elementary assembly parts. Such descriptions can be understood as the semantics of hierarchical assembly sequence plans. They do not have to be provided manually but can be generated automatically. We shall explain this in more detail and will then discuss how this representation scheme applies to different recognition tasks. 


\subsection{Different Syntactic Structures}

It is known from literature that the number of assembly structures does not primarily depend on the number of elementary components but on the way they are interconnected $[55,102,136]$. An assembly of many parts might have only few structures while an assembly of rather few components might come along with numerous assembly sequences. Moreover, two assemblies composed of yet the same objects might differ in their total of component structures (see Fig. 4.1). Thus, as it depends on assembly topology, there is no simple closed form solution to estimate the number of structures from the number of components [136]. One rather has to provide relational models that characterize the contacts among parts and must derive the total of structures therefrom. Dealing with baufix ${ }^{\circledR}$ assemblies this is surprisingly easy.

In Chapter 3.3.1 we saw that a bolted assembly can be characterized as a set of partial ordering relations. It will contain $n$ maximal chains where $n$ is the number of bolts in the assembly. As they consist of a bolt and the sequence of all objects attached to it, the maximal chains represent sets of topologically connected elementary objects. Furthermore, there also are topological relations among the chains. They are pairwise conjoined because objects attached to several bolts appear in several chains. Hence, a bolted assembly corresponds to a topologically connected set of topologically connected sets of elementary objects. The assembly in Fig. 4.1(a), for example, can be modeled by the following set of chains:

$$
\left\{\begin{array}{l}
B O L T_{1} \sqsubseteq_{c_{1}} B A R_{1} \sqsubseteq_{c_{1}} C U B E_{1}, \\
B O L T_{2} \sqsubseteq_{c_{2}} R N U T_{1} \sqsubseteq_{c_{2}} B A R_{1} \sqsubseteq_{c_{2}} C U B E_{2}, \\
B O L T_{3} \sqsubseteq_{c_{3}} C U B E_{1}
\end{array}\right\}
$$

Based on partially ordered sets, disassembly of bolted assemblies can be modeled easily as well. Disassembling a bolted assembly means to loosen a connection between a bolt and a nut. It corresponds to the removal of the maximal element from a chain. In order to end up with stable subassemblies, all miscellaneous objects fixated by this object (which will always be a nut) must be separated, too. As this will yield a set of topologically disjoint subassemblies, disassembly can be understood as a mapping from a set of chains to a set of sets of chains. Detaching $C U B E_{2}$ from $B O L T_{2}$, for instance, would produce three subassemblies collected in the following set:

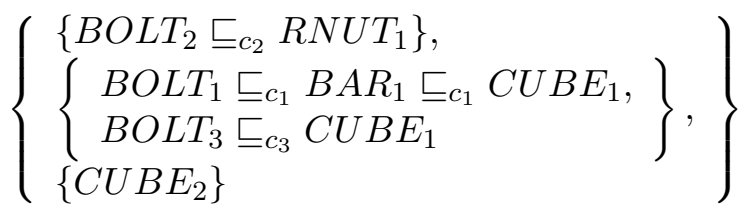

Remember that feasible assembly sequences can be accumulated in an AND/OR graph whose number of trees corresponds to the number of possible syntactic assembly 


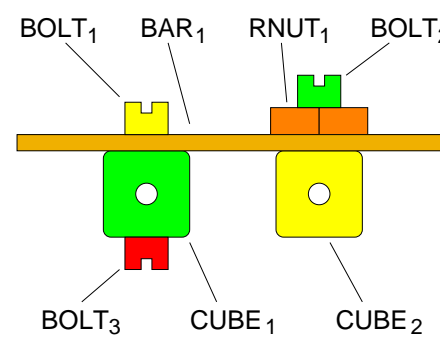

(a) 6 different structures

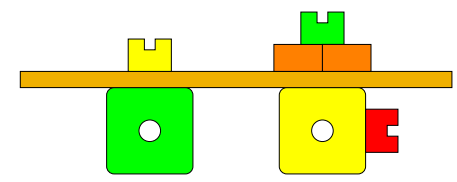

(b) 5 different structures

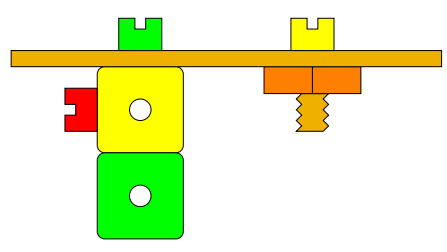

(c) 11 different structures

Figure 4.1.: Sketches of assemblies of different topologies. Though all assemblies consist of three bolts, three nuts, and a bar they differ in the number of possible consists-of structures.

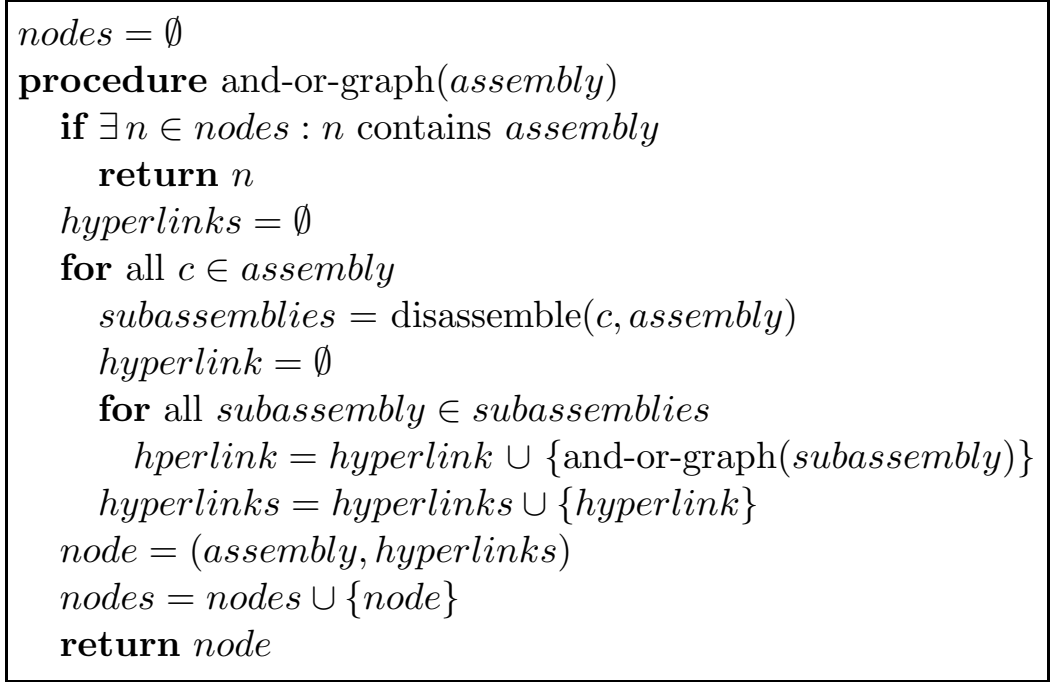

Figure 4.2.: Recursive algorithm to produce the AND/OR graph of a bolted assembly from its representation as a collection of maximal chains of a partially ordered set.

structures. Thus, counting the trees in AND/OR graphs provides a natural approach to assess the complexity of syntactical assembly modeling. Dealing with bolted assemblies this does not even demand considerable implementational effort: as chains of a partially ordered set can be represented as strings, bolted assemblies can be implemented as a collection of strings and disassembly operations can be reduced to ordinary string operations.

Figure 4.2 displays an algorithm that recursively splits collections of strings as exemplified above. The resulting subassemblies are stored in an AND/OR graph which 


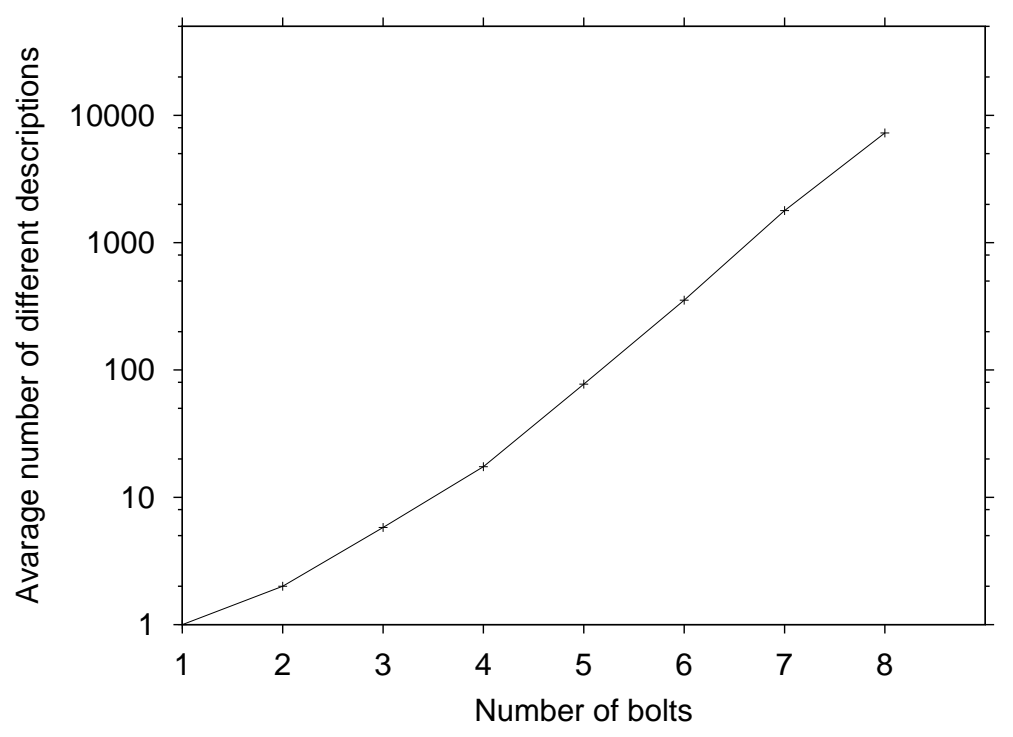

Figure 4.3.: Logarithmic plot of the average number of different syntactic structures of baufix ${ }^{\circledR}$ assemblies with respect to the number of maximal chains (i.e. the number of bolts) contained in an assembly.

is implemented using the recursive data type aog-node(asm) that defines a tuple (asm, $\{\{\operatorname{aog}-\operatorname{node}(\operatorname{asm})\}\}$ ). I.e. the AND/OR graph consists of nodes whose first element contains an assembly while the second one contains a set of hyperlinks where each hyperlink again consists of a set of nodes. Each time the procedure and-or-graph() that generates the graph is called, it verifies whether there already is a node that contains the assembly passed as the parameter. If this is the case, the corresponding node will be returned. If there is no corresponding node yet, a new one will be created and stored in the set of known nodes. It contains the assembly and hyperlinks to all its feasible partitions. To generate the latter, all maximal chains $c$ of the assembly are subjected to a disassemble operation, and-or-graph() is called for each of the resulting subassemblies, and the resulting graphs are collected in a hyperlink.

The number of trees in an AND/OR graph can be estimated from graph traversal along hyperlinks. Since each hyperlink represents an alternative to disassemble the complex object in the node it descends from, alternative hyperlinks must be part of different trees. Thus, whenever top-down traversal reaches a hyperlink whose succeeding nodes all correspond to elementary objects or to assemblies with just one descending hyperlink no alternative branches will appear below and a structural alternative has been found.

Applying this methodology to the baufix ${ }^{\circledR}$ domain led to Fig. 4.3. It logarithmically plots the the average number of alternative syntactic structures against the number of 
bolts contained in an assembly. The curve was determined from counting AND/OR trees of assemblies with up to 8 bolts. For each case we considered 10 assemblies of different topology and averaged the results. As expected from the experiments reported in the literature, the baufix ${ }^{\circledR}$ domain suffers from combinatorial explosion, too. The number of possible assembly sequences and thus of different syntactic structures obviously grows exponentially with the number of assembled bolts.

This result dramatically emphasizes the need for another modeling technique for assembly recognition. Despite of their advantages in assembly detection syntactic descriptions are of little use in recognition. It simply is unreasonable to provide comprehensive databases of individual assembly trees or grammars for flexible and unrestricted recognition by matching syntactic structures ${ }^{1}$. We therefore are in need of another representation which provides unique and comparable descriptions of individual assemblies. These, however, should be easy to provide, simple to handle, and extractable from image data.

\subsection{Graphs as the Semantics of Syntactically Derived Plans}

The key idea to realize a comparable representation of complex objects is to reconsider information yielded from syntactic assembly detection. As explained in Chapter 3.3.1 object cluster parsing results in high-level sequence plans. These plans reflect component structures as well as details of part connections; they uniquely encode topological relations among mating features of elementary assembly components. Moreover, unless detection went wrong, each possible plan of a certain assembly describes the same feature relations. Thus, all possible plans implicitly encode the same relational model.

In order to better understand this, we shall briefly introduce the notion of relational modeling. Afterwards we will see that a special sort of relational models is sufficient to encode mating feature topology and that corresponding models can be derived from high-level assembly sequence plans.

\subsubsection{Relational Modeling}

Following the notation of Vosselman [131], a relational description $D$ is a tuple

$$
D=(P, R)
$$

where $P=\left\{p_{1}, p_{2}, \ldots, p_{N}\right\}$ is a set of primitives and $R$ are relations among those. Each primitive $p_{n}$ is characterized by a set $L$ of attribute value pairs, therefore

$$
p_{n} \in(A \times V)^{L}
$$

\footnotetext{
${ }^{1}$ For the toy airplane, for instance, that contains 11 bolts and is commonly referred as an example when discussing the SFB 360, a total of 1.564 .634 structures was estimated. However, this was impossible using a naive implementation of tree counting. Memory management had to be planned carefully to avoid out of memory errors during computation.
} 
where $A$ is a set of attribute names and $V$ is a corresponding set of symbolic or numeric values. A baufix ${ }^{\circledR}$ object, for instance, could be described by two symbolic attributes: its type and its color and thus might be modeled as

$$
p_{n}=\{(\text { type }, C U B E),(\text { color }, \text { yellow })\} .
$$

Usually, there will be several different relations. For instance, baufix ${ }^{\circledR}$ objects that are located in an assembly cell might be related via relations like in front of or in contact with. And each relation will have several instances. In an assembly, for example, there will be several pairs of objects that form an instance of the in contact with relation. But there might be different contacts between objects. Therefore, an instance of a relation generally consists of a tuple of primitives and has symbolic or numeric attributes as well. Thus, for the $j$ th instance of the $k$ th relation we have

$$
r_{j k} \in P^{M_{k}} \times(A \times V)^{L_{k}} .
$$

All instances of the $k$ th relation are collected in a set $R_{k}$ which is usually paired with a name $N R_{k}$ and thus defines a named relation

$$
P R_{k}=\left(N R_{k}, R_{k}\right) .
$$

A model of a simple assembly that is composed of a bolt, a bar, and a cube, might for instance contain the following named relation

$$
\begin{aligned}
P R_{1}= & \left(\text { in contact with },\left\{\left(p_{1}, p_{2},(\text { contact type }, \text { cylindrical hole insertion })\right)\right.\right. \\
& \left(p_{1}, p_{3},(\text { contact type }, \text { threaded cylindrical hole insertion })\right) \\
& \left.\left.\left(p_{2}, p_{3},(\text { contact type }, \text { planar sur face contact })\right)\right\}\right)
\end{aligned}
$$

where $M_{1}=2, L_{1}=1$, and the $p_{n}$ denote the corresponding objects.

Finally, the relational part of a relational model is given as a set of named relations, i.e.

$$
R=\left\{P R_{1}, P R_{2}, \ldots P R_{K}\right\} .
$$

In their most general form relational descriptions are a mighty paradigm of symbolic knowledge representation. This is why they are long established in structural pattern recognition and image analysis (cf. e.g. $[20,119,131]$ ) as well as in assembly modeling (see Chapter 2). Moreover, it is interesting to note that most approaches to structural knowledge representation are nothing but special cases of relational modeling. An ERNEST knowledge base, for instance, constitutes a relational model for it consists of concepts characterized by attribute value pairs and of different kinds of links relating those concepts. If all relations of a model are binary relations, i.e. each instance of a relation relates exactly two primitives, the model is also called a labeled graph. For the remainder of this chapter we will focus on labeled graphs in assembly modeling and recognition. 


\subsubsection{Labeled Graphs}

Graph theory has a longer tradition than relational modeling. Not surprisingly, it thus has its own terminology. In the following, we will adopt this terminology and base our discussion on definitions by Messmer and Bunke [22, 82, 83]. A directed labeled graph $\Gamma$ is a 4-tuple $\Gamma=(V, E, \mu, \nu)$ where

$V$ is a set of vertices

$E$ is a set of edges where $E \subseteq V \times V$

$\mu$ is a function assigning labels to vertices, i.e. $\mu: V \rightarrow L_{V}$

$\nu$ is a function assigning labels to edges, i.e. $\nu: E \rightarrow L_{E}$

and $L_{V}$ and $L_{E}$ are sets of vertex and edge labels, respectively ${ }^{2}$. From this definition it is obvious that labeled graphs are isomorphic to binary relational models. If we choose $L_{V}=(A \times V)^{L}$, a labeled vertex corresponds to a primitive. And with $L_{E}=(A \times V)^{L_{k}}$ each labeled edge corresponds to a relation $r_{j k} \in R_{k} \subseteq P^{2} \times(A \times V)^{L_{k}}$.

In the following, we will also need the notion of subgraphs. A graph $\Sigma=\left(V^{\prime}, E^{\prime}, \mu^{\prime}, \nu^{\prime}\right)$ is a subgraph of $\Gamma=(V, E, \mu, \nu)$ if

1. $V^{\prime} \subseteq V$

2. $E^{\prime}=E \cap\left(V^{\prime} \times V^{\prime}\right)$

3. $\mu^{\prime}(v)= \begin{cases}\mu(v) & \text { if } v \in V^{\prime} \\ \text { undefined } & \text { otherwise }\end{cases}$

4. $\nu^{\prime}(e)= \begin{cases}\nu(e) & \text { if } e \in E^{\prime} \\ \text { undefined } & \text { otherwise }\end{cases}$

Labeled graphs are widely applied in many fields of computer science. We will concentrate on their application in knowledge representation for image analysis. Here, graph vertices usually represent objects, object parts, or other entities expected in an image while the edges represent known or important relations among them. Bunke [20] points out that the choice of objects, entities, or events represented by the vertices is arbitrary. Except from their suitableness for a given application there are no criteria what primitives and relations should be represented. Knowledge representation based on graphs thus is rather an art that requires experience and careful thought of what information is available and helpful in solving recognition tasks.

After syntactic image analysis, high-level assembly plans provide the most comprehensive information about individual assemblies. We already explained that they describe

\footnotetext{
${ }^{2}$ We denote graphs by $\Gamma$ in order to distinguish them from grammars already termed $G$.
} 
how elementary objects are interconnected and thus provide topologically unique characterizations of baufix ${ }^{\circledR}$ assemblies. Therefore, connection details may be used to recognize assemblies. But part topology is not self-evident from a sequence plan for the connection details are encoded implicitly. Moreover, syntactically different plans of an assembly will encode these relations differently. A labeled graph, however, might explicitly reflect characteristic relations and simultaneously resolve syntactic ambiguities.

Since our general interest lies in compact descriptions and we want to avoid redundancies, the first step towards graph modeling of baufix ${ }^{\circledR}$ assemblies must be to consider what information from syntactic analysis enables recognition and should be modeled. Remember that an assembly sequence plan as introduced in Chapter 3.3.1 directly or indirectly represents

- the elementary objects comprised in the assembly

- a syntactic structure they are organized in

- partial ordering relations among them

- the mating features of objects with holes

- diverse relations among mating features

- angles between bolts attached to the same object

The color of individual assembly parts is not contained in a plan. However, this is a mere question of simplicity. Color information is available from image analysis but would produce illegible plans if annotated. In the following, we will assume it to be given.

As we already know, the first three pieces of information in the above list are less specific than the following ones: a given set of objects might be assembled into many configurations (see Fig. 4.1) and a single configuration might have different syntactic structures. But different configurations involve different mating features and there will be different feature relations. Thus, relations between mating features comprehensively characterize how objects are interconnected; they determine the topology of an assembly. This directly leads to the idea of a graph representation that captures the relations among mating features of elementary objects.

After choosing mating features to be the primitives of a graph based model for assembly recognition, we still need suitable attributes to discriminate them. In the baufix ${ }^{\circledR}$ domain, a mating feature can, for instance, be characterized by its type and by the kind and color of the object it is part of. Consequently, we choose the range of the vertex labeling function $\mu$ as follows

$$
\mu: V \rightarrow T \times O \times C
$$

where

$$
T=\{\text { HOLE, THREAD }, \ldots\}
$$




$$
\begin{aligned}
& O=\{\text { BOLT }, \text { CUBE, RNUT, } \ldots\} \\
& C=\{\text { red, orange, yellow, green, } \ldots\} .
\end{aligned}
$$

Our choice of significant relations among mating features is based on the following considerations. Modeling an assembly of several objects by means of the corresponding mating features is rather abstract. The degree of abstraction will be reduced, if features belonging to the same object are clustered so that object structures become visible. This could be accomplished by drawing edges from each mating feature of a certain object to all its other features. For an object with $n$ mating features, however, this would introduce $n(n-1)$ edges and thus might require unreasonable modeling effort. Instead, it would suffice to relate vertices of topologically adjacent features and label them respectively. This will produce sets of interconnected vertices and yield the desired clustering.

Connections between features from different parts may be expressed using partial ordering relations. In Chapter 3.3.1 we pointed out that the components of a bolted attachment are linearly arranged in space. This also holds for the involved mating features and on page 52 we actually exploited that to extract connection details from image data. The resulting plans implicitly reflect these feature sequences as well: seen as a tree the syntactic structure of a sequence plan is such that elementary bolts will always be the leftmost leaf of a subtree. And if the tree is traversed from left to right, the objects and thus the features attached to a certain bolt will appear in the sequence they were put onto it. Drawing edges between vertices representing successive elements of such sequences yields chains of mating features and characterizes the topology of bolted part connections.

Finally, sequence plans like shown in Fig. 4.4(b) indirectly encode angles between bolts attached to the same object. Slots representing used holes of a bar are labeled with the corresponding bolt and with a flag indicating the side the bolt was inserted from. Hence, if there are several bolts attached to a bar, those with the same flag will be oriented parallel while those with different flags will be anti-parallel. And as the features of cubes are counted in a clockwise manner each pair of bolts attached to a cube can easily be labeled as perpendicular or anti-parallel. Actually, this summarized all possible relative orientations or angles between bolts. In the baufix ${ }^{\circledR}$ domain, two bolts attached to the same object are either parallel $\left(0^{\circ}\right)$, perpendicular $\left(90^{\circ}\right)$, or anti-parallel $\left(180^{\circ}\right)$.

Therefore, by choosing the range of the edge labeling function $\nu$ to be

$$
\nu: E \rightarrow\left\{\mathrm{ADJ}, \sqsubseteq, 0^{\circ}, 90^{\circ}, 180^{\circ}\right\}
$$

comprehensive topological assembly knowledge can be represented in a mating feature graph.

The following example should illustrate what we are typically dealing with in practice. Figure 4.4(c) depicts the mating feature graph derived from the plan in Fig. 4.4(b). Its vertices represent subparts of elementary objects that are part of the assembly in 


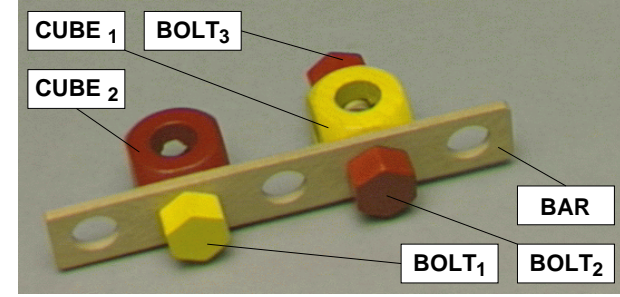

(a)

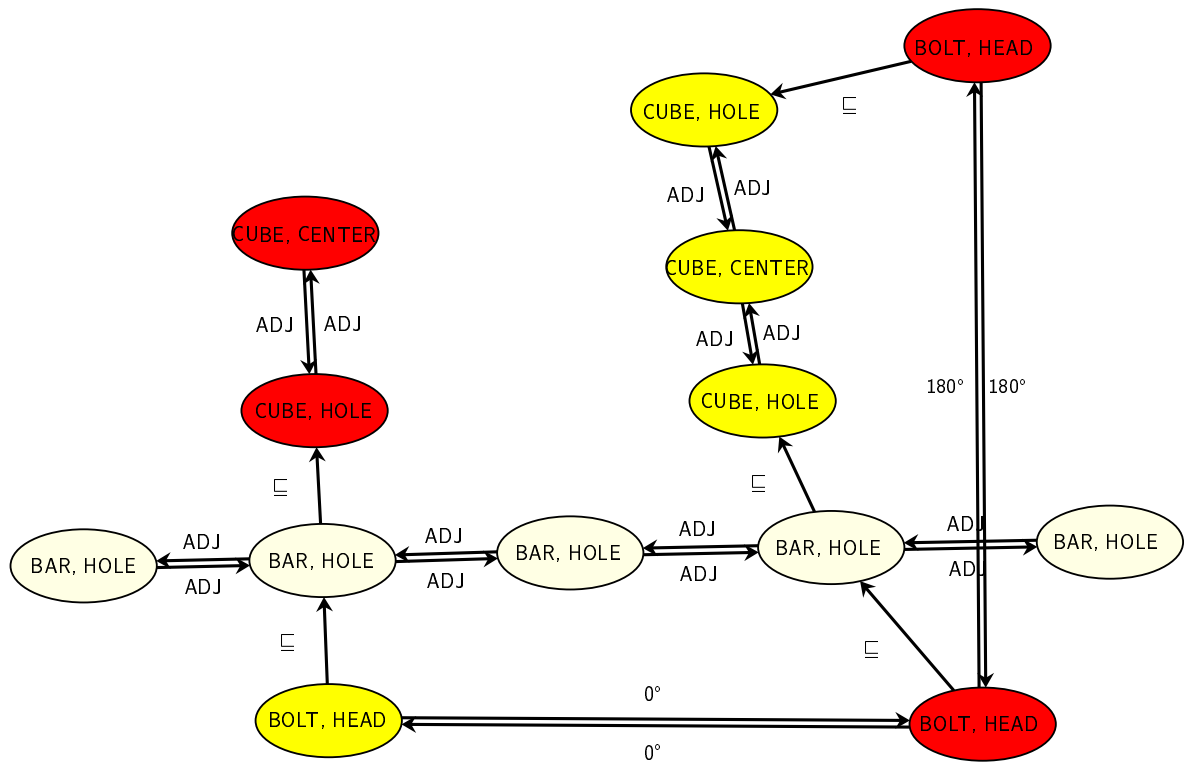

(c)

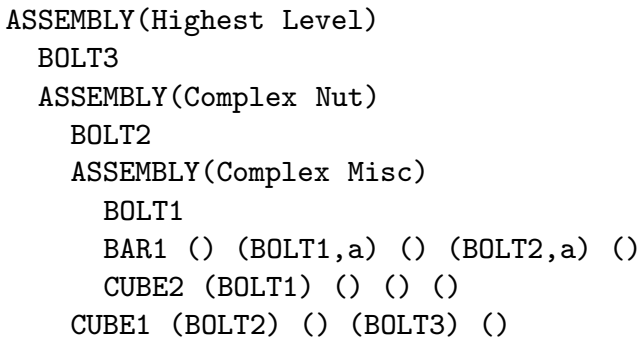

(b)

Figure 4.4.: 4.4(a) An exemplary assembly known from the second chapter. 4.4(b) A corresponding high-level sequence plan resulting from syntactic image analysis. 4.4(c) A graph describing relations among the mating features or subparts of the assembly. Subparts related by edges labeled 'ADJ' belong to the same object. Edges labeled ' $\sqsubseteq$ ' indicate sequences of objects attached to a bolt and edges labeled with an angle represent the relative orientation between bolts attached to the same object.

Fig. 4.4(a). Each vertex is labeled with a feature type and with the type of object it belongs to. Furthermore, vertices are colored according to the object they are part of. 
Subparts connected via pairs of edges labeled 'ADJ' belong to the same object. Thus, certain subgraphs of the graph represent elementary baufix ${ }^{\circledR}$ objects: the bar is represented by a cluster of five holes while the cubes are represented by their occupied holes and by their center of mass. The latter is due to the information available. As explained on page 52 , the coordinates of a cube hole are computed from a geometric heuristic. Since it only allows to determine coordinates of holes connected to a bolt, we decided to regard the center of a cube as a virtual mating feature in order to provide further information. Bolts are represented by their heads. These are no real mating features but their threads will seldom be visible. And furthermore, heads of bolts are natural minimal elements of partial orders that represent mating relations between objects. Chains of edges labeled ' $\sqsubseteq$ ' indicate sequences of subparts attached to a certain bolt. Finally, for each pair of bolts connected to the same object, the corresponding 'BOLT HEAD' vertices are related via edges labeled with the angle the bolts enclose.

From the components of the graph we now can recognize the topological structure of the exemplary assembly. The 'ADJ' relations subdivide the graph into six clusters which represent the assembly's elementary parts. The ' $\sqsubseteq$ ' relations indicate that the yellow bolt is attached to the bar and the red cube and that one of the red bolts is attached to the bar and the yellow cube while the other is only assembled with the cube. We also see that the bolts attached to the bar are inserted into the holes on the left and right of the its center. As these bolts are related by edges labeled ' $0{ }^{\circ}$ ' we can further conclude that they were inserted from the same direction. Finally, the angle of $180^{\circ}$ between the bolts assembled with the yellow cube indicates that these are facing each other.

\subsubsection{Computing Mating Feature Graphs}

All feasible sequence plans of an assembly have the same meaning. They all describe the assembly's part topology which is uniquely determined by relations among mating features. Therefore, mating feature graphs uniquely representing these relations can be seen as the semantics of assembly sequence plans.

Semantics is the study of meaning. Apart from other disciplines, it plays a prominent role in several fields of computer science. In the theory of programming languages, for instance, it describes the meaning of programs. A common way to do this is called denotational semantics [36, 122]. Within a denotational semantics framework, programs are interpreted as functions over abstract mathematical objects and the syntactic components of a program are mapped to mathematical entities. These might be numbers, truth values, functions, etc. However, in order to avoid inconsistencies which might arise from the interpretation of recursive structures, the theory demands that the range of a denotational semantics constitutes a so called semantic domain.

All this resembles our situation since plans seem to correspond to programs and graphs are mathematical objects. Yet to completely justify the notion of mating feature 
graphs as the semantic of plans, we must show that assembly sequence plans really are syntactic entities and that mating feature graphs are elements of a semantic domain.

\section{The Syntax of Assembly Sequence Plans}

So far, except for their hierarchical nature we hardly cared about the structure of plans like shown in Fig. 4.4(b). But they actually are words of a context free language: given an alphabet of terminal symbols

$$
\Sigma=\{\operatorname{ASSEMBLY}(, \text { BOLT }, \text { CUBE, RNUT, } \ldots,(\text { BOLT },),,,(), \mathrm{a}, \mathrm{b}, 0,1, \ldots, 9, \text { HighestLevel }, \ldots\},
$$

a set of non terminals

$$
N=\{\text { cmplxobj, boltobj, nutobj, holes, barholes, fnct, side, num }\},
$$

and the following context free productions (presented in an EBNF notation)

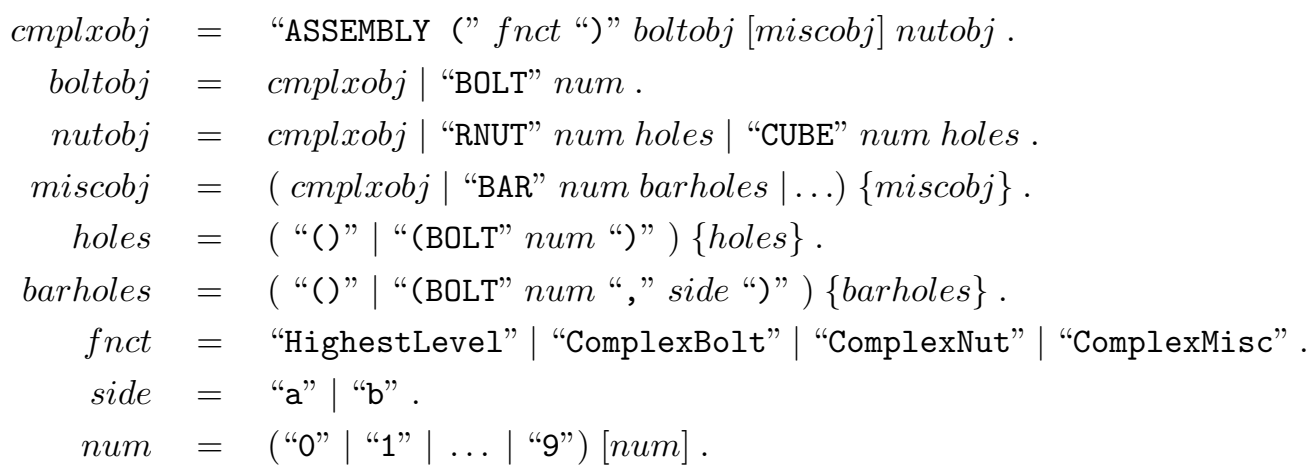

the feasible sequence plans for baufix ${ }^{\circledR}$ assemblies form a subset of the language

$$
\{x \mid \text { cmplxobj } \stackrel{*}{\Rightarrow} x\} .
$$

Choosing cmplxobj as the start symbol, the grammar generates words whose general structure corresponds to that of bolted assemblies: cmplxobj derives the variables boltobj, miscobj, and nutobj which in turn either derive terminal symbols representing elementary objects or produce another complex object. But the grammar also generates detailed structures known from Chapter 3.3.1. Each terminal representing a baufix ${ }^{\circledR}$ object is followed by a number that allows to identify the object and several objects appear with (lists of) parentheses which may enclose terminals denoting bolts. Of course there will be derivations that do not correspond to feasible assembly plans. The number of parentheses associated with an object is unrestricted and there is no mechanism that guarantees consistent assignments of bolts to other objects. However, each feasible and correct plan can be derived from this grammar. Therefore, assembly plans resulting from image analysis are regularly composed from syntactic units which can be mapped to mathematical objects; the overall meaning of a plan will be composed of these. 


\section{Mating Feature Graphs as a Semantic Domain}

In order to verify that mating features graphs constitute a semantic domain, we shall first define this term. A structure $\mathcal{A}=\left(A, \sqsubseteq_{A}\right)$ is a complete partial order or a semantic domain if

1. $A$ is a partially ordered set with respect to $\sqsubseteq_{A}$

2. there is a minimal element $\perp \in A$ such that for all $a \in A, \perp \sqsubseteq_{A} a$

3. each chain $C \subseteq A$ has a least upper bound $\sqcup C$ in $A$.

Given this definition it is easy to see that mating feature graphs indeed form a semantic domain. Consider the infinite set

$$
M F G=\{\Gamma \mid \Gamma \text { is a mating feature graph }\} \cup\{\perp\}
$$

consisting of all possible mating feature graphs and the empty graph $\perp=(\emptyset, \emptyset, \Omega, \Omega)$ where $\Omega$ denotes a totally undefined partial function. If we define

$$
\Gamma_{1} \sqsubseteq_{M F G} \Gamma_{2} \Leftrightarrow \Gamma_{1} \text { is subgraph of } \Gamma_{2}
$$

with $\Gamma_{1}, \Gamma_{2} \in M F G$, then $\mathcal{M F \mathcal { G }}=\left(M F G, \sqsubseteq_{M F G}\right)$ is a semantic domain.

\section{A Denotational Semantics of Assembly Sequence Plans}

Now that we know that high-level assembly sequence plans are thoroughly structured by a grammar and that mating feature graphs can be organized in a semantic domain, we will present a semantics function $\mathcal{S}$ that computes graphs from plans. To this end we will employ the mathematical notation known from literature. A semantics function will be written

$$
\mathcal{F} \llbracket s \rrbracket a_{1} a_{2} \ldots a_{n}
$$

where $\mathcal{F}$ is the name of the function, $s$ enclosed by semantics brackets is a sequence of symbols, i.e. the main argument of the function, and the $a_{i}$ are further arguments necessary to compute the semantics of $s^{3}$. To increase readability we make use of the convention

$$
\mathcal{F} \llbracket s \rrbracket \mathcal{G} \llbracket t \rrbracket a_{1} a_{2} \ldots a_{n} \Leftrightarrow \mathcal{F} \llbracket s \rrbracket\left(\mathcal{G} \llbracket t \rrbracket a_{1} a_{2} \ldots a_{n}\right) .
$$

Finally, given a partial function $f: A \leadsto B$, the function $f\left[\frac{i}{x}\right]: A \leadsto B$ is defined by

$$
f\left[\frac{i}{x}\right](j)= \begin{cases}x & \text { if } i=j \\ f(j) & \text { if } i \neq j .\end{cases}
$$

\footnotetext{
${ }^{3}$ Note that if $\mathcal{A}_{1}, \mathcal{A}_{2}, \ldots \mathcal{A}_{n}$ are semantic domains, $\mathcal{A}_{1} \times \mathcal{A}_{2} \times \ldots \times \mathcal{A}_{n}$ is a semantic domain, too.
} 
Now we are prepared to take a look at $\mathcal{S}$. Remember that high-level sequence plans consist of symbols from an alphabet $\Sigma$. Since only certain words over $\Sigma$ correspond to feasible and correct plans, $\mathcal{S}$ will be a partial function

$$
\mathcal{S}: \Sigma^{*} \leadsto M F G \text {. }
$$

Provided a feasible plan, $\mathcal{S}$ should yield a graph whereas its behavior does not have to be defined for arbitrary arguments. We already mentioned that the semantics of a complex structure is usually composed from the meanings of its parts. Furthermore, the meaning of a part might depend on earlier computations. Considering this we define $\mathcal{S}$ as follows:

$$
\mathcal{S} \llbracket p \rrbracket=\operatorname{partS} \llbracket p \rrbracket \perp
$$

where $\perp$ denotes the empty mating feature graph. Thus, computing the semantics of a plan $p$ means to compute a function part $\mathcal{S}$ that is applied to $p$ and to the empty graph. This implicitly settles its type:

$$
\text { partS : } \Sigma^{*} \times M F G \leadsto M F G .
$$

Defining part $\mathcal{S}$ will be a little burdensome for its result will certainly depend on the form of the argument $p$. As it would be fruitless and too laborious to discuss the definition in full length, we will present just a few cases ${ }^{4}$. First, let us consider the semantics of a whole plan, i.e. of a sequence of symbols headed by ASSEMBLY:

$$
\operatorname{partS} \llbracket \operatorname{ASSEMBLY}(f) B M_{1} \ldots M_{n} N \rrbracket \Gamma=\operatorname{part} \mathcal{S} \llbracket N \rrbracket \ldots \operatorname{part} \mathcal{S} \llbracket M_{1} \rrbracket \operatorname{part} \mathcal{S} \llbracket B \rrbracket \Gamma
$$

Given a mating feature graph $\Gamma$, the semantics of an assembly (plan) composed of a bolt-part $B$, a couple of miscellaneous-parts $M_{i}$, and a nut part $N$ is composed from the semantics of these components. Hence, partS is consecutively called for each part. While the first call depends on $\Gamma$ each of the following calls will depend on the result of the preceding computation. Note that this definition is recursive for the parts $B, M_{1}, \ldots, N$ might represent complex objects and thus lead to further calls of partS $\llbracket$ ASSEMBLY ... $\rrbracket$.

The semantics of an elementary plan element denoting a bolt is defined to be:

$$
\operatorname{partS} \llbracket \operatorname{BOLT} n \rrbracket(V, E, \mu, \nu)=\left(V^{\prime}, E, \mu^{\prime}, \nu\right)
$$

where

$$
\begin{aligned}
V^{\prime} & =V \cup\left\{\operatorname{vrtx}\left(\operatorname{BOLT}_{n}\right)\right\} \\
\mu^{\prime} & =\mu\left[\frac{\operatorname{vrtx}\left(\operatorname{BOLT}_{n}\right)}{\left(\operatorname{BOLT}^{\prime} \operatorname{HEAD}, \operatorname{col}\left(\mathrm{BOLT}_{n}\right)\right)}\right] .
\end{aligned}
$$

\footnotetext{
${ }^{4} \mathrm{~A}$ complete definition of partS and its subfunctions can be found in Appendix D; it is a revised and more elegant version than the one originally presented in [15].
} 
Thus, partS applied to a bolt and a graph adds a new vertex to the graph and modifies its functions that assigns labels to vertices. If we assume the vertex to be identified by a constructor $\operatorname{vrtx}\left(\mathrm{BOLT}_{n}\right)$, the function $\mu$ will have to be modified such that it maps $\operatorname{vrtx}\left(\mathrm{BOLT}_{n}\right)$ onto a triple of attributes (BOLT,HEAD, $\left.\operatorname{col}\left(\mathrm{BOLT}_{n}\right)\right)$. Corresponding to our initial consideration regarding mating feature graphs of baufix ${ }^{\circledR}$ assemblies, the new vertex thus represents the head of a colored bolt where the corresponding color information is assumed to be obtainable from a function $\operatorname{col}()$. All other vertices from $V^{\prime}$ remain unchanged. ${ }^{5}$

The semantics of plan elements denoting objects with a single hole is slightly more complex for they are related to a bolt. As an example, let us consider the plan semantics of a rhomb-nut:

$$
\text { partS } \llbracket \operatorname{RNUT} n H \rrbracket \Gamma=\text { rnutholeS } \mathbb{S} \llbracket H \rrbracket n \Gamma
$$

where the function rnuthole $\mathcal{S}$ has the type

$$
\text { rnutholeS : } \Sigma^{*} \times \mathbb{N} \times M F G \leadsto M F G
$$

and is defined as follows:

$$
\text { rnuthole } \mathcal{S} \llbracket(\text { BOLT } m) \rrbracket n(V, E, \mu, \nu)=\left(V^{\prime}, E^{\prime}, \mu^{\prime}, \nu^{\prime}\right)
$$

where

$$
\begin{aligned}
V^{\prime} & =V \cup\left\{\operatorname{vrtx}\left(\operatorname{RNUT}_{n}\right)\right\} \\
E^{\prime} & =E \cup\left\{\left(v, \operatorname{vrtx}\left(\operatorname{RNUT}_{n}\right)\right)\right\}, v \in V \wedge \operatorname{vrtx}\left(\mathrm{BOLT}_{m}\right) \sqsubseteq v \wedge \nexists v^{\prime} \in V: v \sqsubseteq v^{\prime} \\
\mu^{\prime} & =\mu\left[\frac{\operatorname{vrtx}\left(\mathrm{RNUT}_{n}\right)}{\left(\mathrm{RNUT}_{n}, \mathrm{HOLE}_{\text {,orange }}\right)}\right] \\
\nu^{\prime} & =\nu\left[\frac{\left(v, v r t x\left(\mathrm{RNUT}_{n}\right)\right)}{\sqsubseteq}\right] .
\end{aligned}
$$

Given a graph, rnutholeS introduces a new vertex and a new edge and modifies the labeling functions respectively. The label of the new vertex indicates that it represent the hole of a rhomb-nut. Note that all baufix ${ }^{\circledR}$ rhomb-nuts are orange so the color information can be passed as a constant. The new edge $\left(v, \operatorname{vrtx}\left(\mathrm{RNUT}_{n}\right)\right)$ expresses that $\mathrm{RNUT}_{n}$ is attached to $\operatorname{BOLT}_{m}$. It is drawn between the last element $v$ in the chain of vertices partially ordered due to $\mathrm{BOLT}_{m}$ and is assigned a label ' $\sqsubseteq$ '.

The semantics of plan elements representing objects of multiple holes is even more complex. Cubes or bars might be related to several bolts and, accordingly, those bolts will be interrelated as well. The semantics of a bar, for instance, is given by:

$$
\begin{aligned}
& \text { part } \mathcal{S} \llbracket \operatorname{BAR} n H_{1} \ldots H_{m} \rrbracket \Gamma= \\
& \text { interpartS } \llbracket \operatorname{BAR} n H_{1} \ldots H_{m} \rrbracket \text { intrapart } \mathcal{S} \llbracket \operatorname{BAR} n \rrbracket \text { barhole } \mathcal{S} \llbracket H_{m} \rrbracket n m \ldots \text { barhole } \mathcal{S} \llbracket H_{1} \rrbracket n 1 \Gamma
\end{aligned}
$$

\footnotetext{
${ }^{5}$ For reasons of simplicity we assumed that symbols like $n$ that are derivable from the variable num always denote a number and that the symbol and the number may be used synonymously. We will keep this assumption henceforth.
} 
It is computed from consecutive applications of a function calculating the semantics of the holes of the bar and subsequent calls of functions that calculate relations between the holes and relations between bolts attached to the bar. The function barholeS that calculates the semantics of a hole is typed:

$$
\text { barhole } \mathcal{S}: \Sigma^{*} \times \mathbb{N} \times \mathbb{N} \times M F G \leadsto M F G \text {. }
$$

For an empty pair of parentheses denoting a hole not attached to any bolt it is defined as follows:

$$
\text { barhole } \mathcal{S} \llbracket() \rrbracket i j(V, E, \mu, \nu)=\left(V^{\prime}, E, \mu^{\prime}, \nu\right)
$$

where

$$
\begin{aligned}
& V^{\prime}=V \cup\left\{\operatorname{vrtx}\left(\mathrm{BAR}_{i j}\right)\right\} \\
& \mu^{\prime}=\mu\left[\frac{v r t x\left(\mathrm{BAR}_{i j}\right)}{(\mathrm{BAR}, \mathrm{HOLE}, \text { wood })^{\prime}}\right] .
\end{aligned}
$$

I.e. in this case barhole $\mathcal{S}$ will generate a new vertex and a corresponding label. If barhole $\mathcal{S}$ is called for a hole that is attached to a bolt, we have:

$$
\text { barholeS } \llbracket(\text { BOLT } m, s) \rrbracket i j(V, E, \mu, \nu)=\left(V^{\prime}, E^{\prime}, \mu^{\prime}, \nu^{\prime}\right)
$$

where

$$
\begin{aligned}
& V^{\prime}=V \cup\left\{\operatorname{vrtx}\left(\mathrm{BAR}_{i j}\right)\right\} \\
& E^{\prime}=E \cup\left\{\left(v, \operatorname{vrtx}\left(\mathrm{BAR}_{i j}\right)\right)\right\}, v \in V \wedge \operatorname{vrtx}\left(\mathrm{BOLT}_{m}\right) \sqsubseteq v \wedge \nexists v^{\prime} \in V: v \sqsubseteq v^{\prime} \\
& \mu^{\prime}=\mu\left[\frac{v r t x\left(\mathrm{BAR}_{i j}\right)}{(\mathrm{BAR}, \mathrm{HOLE}, \text { wood })}\right] \\
& \nu^{\prime}=\nu\left[\frac{\left(v, v r t x\left(\mathrm{BAR}_{i j}\right)\right)}{\sqsubseteq}\right] \text {. }
\end{aligned}
$$

Hence, for an occupied hole of a bar a vertex and an edge will be introduced analogous to the rhomb-nut case.

Adjacency relations between holes are computed by the function intrapart $\mathcal{S}$ whose type is given by:

$$
\text { intrapartS : } \Sigma^{*} \times M F G \leadsto M F G \text {. }
$$

If applied to a plan element BAR $n$ and a graph, it is defined to be:

$$
\text { intrapartS } \llbracket \operatorname{BAR} n \rrbracket(V, E, \mu, \nu)=\left(V, E^{\prime}, \mu, \nu^{\prime}\right)
$$

where

$$
\begin{aligned}
E^{\prime}= & E \cup E_{\text {intra }} \\
\nu^{\prime}= & \nu\left[\frac{e}{\mathrm{ADJ}}\right], \forall e \in E_{\text {intra }} \\
V_{B_{n}}= & \left\{\operatorname{vrtx}\left(\mathrm{BAR}_{i j}\right) \in V \mid i=n \wedge j \in \mathbb{N}\right\} \\
E_{\text {intra }}= & \left\{\left(\operatorname{vrtx}\left(\mathrm{BAR}_{n j}\right), \operatorname{vrtx}\left(\mathrm{BAR}_{n j+1}\right)\right),\left(\operatorname{vrtx}\left(\mathrm{BAR}_{n j+1}\right), \operatorname{vrtx}\left(\mathrm{BAR}_{n j}\right)\right) \mid\right. \\
& \operatorname{vrtx}\left(\mathrm{BAR}_{n j}, \operatorname{vrtx}\left(\mathrm{BAR}_{n j+1} \in V_{B_{n}} \wedge j=1, \ldots,\left|V_{B_{n}}\right|-1\right\}\right.
\end{aligned}
$$


intrapartS thus introduces a set of new edges and corresponding labels to the graph passed in the argument. The set $V_{B_{n}}$ contains all vertices that were generated from preceding calls of barholeS for the holes of BAR $n$. In the set $E_{\text {intra }}$ the vertices from $V_{B_{n}}$ are paired such that vertices representing adjacent holes of the bar are related by an edge. And each edge in this set is assigned a label 'ADJ'.

Finally, the function interpart $\mathcal{S}$ of type

$$
\text { interpartS : } \Sigma^{*} \times M F G \leadsto M F G
$$

computes relations among bolts attached to the same bar. It is defined as follows:

$$
\text { interpartS } \llbracket \operatorname{BAR} n H_{1} \ldots H_{m} \rrbracket(V, E, \mu, \nu)=\left(V, E^{\prime}, \mu, \nu^{\prime}\right)
$$

where

$$
\begin{aligned}
E^{\prime} & =E \cup\left\{e \mid\left(e,\left(s, s^{\prime}\right)\right) \in P_{2}\right\} \\
\nu^{\prime} & =\nu\left[\frac{e}{\varphi\left(s, s^{\prime}\right)}\right], \forall\left(e,\left(s, s^{\prime}\right)\right) \in P_{2} \\
P_{1} & =\left\{\left(\operatorname{vrtx}\left(\mathrm{BOLT}_{m}\right), s\right) \mid \operatorname{vrtx}\left(\mathrm{BOLT}_{m}\right) \in V \wedge\left(\mathrm{BOLT}_{m}, s\right) \in\left\{H_{1} \ldots H_{m}\right\}\right\} \\
P_{2} & =\left\{\left(\left(v, v^{\prime}\right),\left(s, s^{\prime}\right)\right) \mid(v, s),\left(v^{\prime}, s^{\prime}\right) \in P_{1} \wedge v \neq v^{\prime}\right\} .
\end{aligned}
$$

The vertices of bolts attached to the holes of $\operatorname{BAR} n$ are paired with the flag that indicates from which side the bolt was inserted into the bar; these pairs are collected in the set $P_{1}$. Then each two pairs from $P_{1}$ are transformed into a tuple whose first component contains two vertices (i.e. an edge) while the second component contains the corresponding flags. These form the set $P_{2}$ where edges between bolts and the enclosed angles are computed from: for each element in $P_{2}$ the edge in the first component is labeled with the result of a function $\varphi$ applied to the flags from the second component. In accordance with the baufix ${ }^{\circledR}$ domain $\varphi$ is defined to be:

$$
\varphi\left(s, s^{\prime}\right)= \begin{cases}0^{\circ}, & \text { if } s=s^{\prime} \\ 180^{\circ}, & \text { otherwise. }\end{cases}
$$

\subsubsection{Intermediate Summary}

As the last sections descended rather deep into mathematical details of semantics theory, the overall thread of this chapter might have been lost. In order to prevent this danger, we will briefly summarize their general topics and results.

The observation which led to the discussion of relational models, labeled graphs, and their generation was that high-level sequence plans for assembled objects implicitly describe topological relations among assembly components. Furthermore, though an assembly might have numerous plans of different structure, they all will describe the same object and thus will encode the same relations. Hence, based on relational descriptions assembly recognition by means of structural matching would require far less models as if it was based on syntactic descriptions. 
Relations among a set of entities can be explicitly represented in a relational model. Labeled graphs are a special kind of relational models which seems appropriate to capture the relevant information encoded in assembly plans. And in fact, we presented a class of graphs, which we called mating feature graphs, that uniquely describes the topology of baufix ${ }^{\circledR}$ assemblies.

Then we discussed if it was possible to derive this explicit representation from the information implicitly provided by assembly plans. As different plans of an assembly all yield the same relations, we asked whether mating feature graphs could be considered as the semantics of plans. We saw that this is possible. On the one hand, high-level sequence plans as presented in Chapter 3.3.1 are thoroughly syntactically structured and thus may be argument of a semantics functions. On the other hand, paired with a suitable partial ordering relation, mating feature graphs form a semantic domain. Thus, the prerequisites to define a semantics function are given and we presented one.

Though this mathematical elaboration seems burdensome and of little practical relevance, it has two considerable advantages. It revealed yet another similarity to the theory of formal languages. While Chapter 3 showed that syntactic methods are well suited to structure assemblies, the last sections proved that semantics theory provides a framework to interpret syntactic assembly structures. And due to the generality of the theoretical background we can assume that the presented method not only copes with baufix ${ }^{\circledR}$ assemblies but that there will be corresponding models and interpretations for other domains as well.

\subsection{Matching Mating Feature Graphs}

Using graphs to represent object knowledge for a recognition task is well established in computer vision (cf. e.g. $[21,120]$ and the references therein). Given graph based representations object recognition becomes a problem of graph matching.

Graph matching is about finding isomorphisms between graphs. A bijective function $f: V \rightarrow V^{\prime}$ is called a graph isomorphism from a graph $\Gamma=(V, E, \mu, \nu)$ to a graph $\Gamma^{\prime}=\left(V^{\prime}, E^{\prime}, \mu^{\prime}, \nu^{\prime}\right)$ if

1. $\mu(v)=\mu^{\prime}(f(v))$ for all $v \in V$

2. $\forall e=\left(v_{1}, v_{2}\right) \in E: \exists e^{\prime}=\left(f\left(v_{1}\right), f\left(v_{2}\right)\right) \in E^{\prime}: \nu(e)=\nu^{\prime}\left(e^{\prime}\right)$ and $\forall e^{\prime}=\left(v_{1}^{\prime}, v_{2}^{\prime}\right) \in E^{\prime}: \exists e=\left(f^{-1}\left(v_{1}^{\prime}\right), f^{-1}\left(v_{2}^{\prime}\right)\right) \in E: \nu^{\prime}\left(e^{\prime}\right)=\nu(e)$

An injective function $f: V \rightarrow V^{\prime}$ is called a subgraph isomorphism from $\Gamma$ to $\Gamma^{\prime}$ if there is a subgraph $\Sigma$ of $\Gamma^{\prime}$ such that $f$ is graph isomorphism from $\Gamma$ to $\Sigma$.

The applicability of the concept of subgraph isomorphism to pattern recognition and computer vision is obvious. Suppose there is a collection of model graphs each of which represents an object or an entity expected to be found in a sensor signal and suppose there is an input graph that was derived from a signal. If a subgraph isomorphism from any of 
the model graphs to the input graph can be detected, the input graph will correspond to or contain the respective model. In other words, the entity represented by the model graph will have been recognized in the signal.

However, signals or images taken by a sensor or a camera will usually be corrupted by noise or suffer from occlusion or other kinds of distortions. Therefore, graph representations derived from a signal usually cannot be guaranteed to match any of the model graphs. In order to increase robustness and to yet enable graph based recognition, various approaches to the detection of error-correcting subgraph isomorphisms have been developed (cf. $[82,83]$ and the references therein). One idea was to search for similar graphs by computing a distance.

A popular measure to asses the similarity of two graphs is to compute their edit distance. To this end a number of graph edit operations is defined that may be used to alter one of the graphs until a subgraph isomorphism can be detected. Given a graph $\Gamma=(V, E, \mu, \nu)$, Messmer and Bunke [82, 83] define a graph edit operation $\delta$ on $\Gamma$ to be any of the following five operations:

$$
\begin{aligned}
& \text { vertex label substitution: } \mu(v) \rightarrow l, v \in V, l \in L_{V} \\
& \text { edge label substitution: } \nu(e) \rightarrow l^{\prime}, e \in E, l^{\prime} \in L_{E} \\
& \text { vertex deletion: } v \rightarrow \$, v \in V \text { where edges } e \in E \text { incident with } v \text { are deleted, too } \\
& \text { edge deletion: } e \rightarrow \$, e \in E \\
& \text { edge insertion: } \$ \rightarrow e=\left(v_{1}, v_{2}\right), v_{1}, v_{2} \in V
\end{aligned}
$$

As the edited graph will usually be the model graph and one usually is looking for subgraph isomorphisms from the model to the input graph, the insertion of vertices has not to be considered because extraneous vertices in the input graph will be ignored, anyway. Defined as above, it will always be possible to specify a sequence $\Delta=\delta_{1} \delta_{2} \ldots \delta_{n}$ of edit operations that transforms any graph $\Gamma$ into a subgraph of any other graph $\Gamma^{\prime}$. Thus, each model graph $\Gamma_{M}$ can be transformed in a graph $\Delta\left(\Gamma_{M}\right)$ such that a subgraph isomorphism $f_{\Delta}$ from $\Delta\left(\Gamma_{M}\right)$ to an input graph $\Gamma_{I}$ exists. The tuple $\left(\Delta, f_{\Delta}\right)$ is called an error-correction subgraph isomorphism from $\Gamma_{M}$ to $\Gamma_{I}$.

To determine the graph edit distance between $\Gamma$ and $\Gamma^{\prime}$ a cost $C(\delta)$ is assigned to each type of edit operation. However, these costs are strongly depend on the application one is dealing with and must be estimated from experience or according to some heuristic. The cost of a sequence of edit operations then simply is

$$
C(\Delta)=\sum_{i=1}^{n} C\left(\delta_{i}\right) .
$$

And the edit distance between two graphs $\Gamma$ and $\Gamma^{\prime}$ is the minimum cost taken over all sequences $\Delta$ that transform $\Gamma$ into $\Gamma^{\prime}$. Finally, in graph matching primary interest lies 
not in the edit distance between $\Gamma$ and $\Gamma^{\prime}$ but in their subgraph distance. This is defined to be the minimum cost taken over all error-correcting subgraph isomorphisms from $\Gamma$ to $\Gamma^{\prime}$; the error-correcting subgraph isomorphism associated with the subgraph distance is called the optimal error-correcting subgraph isomorphism.

Even if all all these definitions are reasonable and easy to understand, detecting subgraph isomorphisms is not a simple problem. Generally, its solution will be computationally expensive because finding ordinary as well as error-correcting isomorphisms between two graphs is known to be $N P$ complete. As this obviously complicates the detection of isomorphisms between a set of model graphs and an input graph, much effort has been spent in the development of efficient algorithms for matching graphs against a database of models (cf. [82, 83] and the references therein). Since this thesis is first of all concerned with practical problems of visual assembly recognition in a flexible environment, it would have been far beyond its scope to develop an algorithm that efficiently matches mating feature graphs. In the following, we will thus describe the use of the graph matching toolkit of the University of Berne (GUB). It was developed by Messmer and Bunke $[82,83]$ and implements several algorithms for detecting errorcorrecting subgraph isomorphisms from a collection of model graphs to an input graph. We shall discuss its application to the recognition of individual baufix ${ }^{\circledR}$ assemblies and to $3 \mathrm{D}$ assembly reconstruction from stereo vision.

\subsubsection{Assembly Recognition}

A pleasant advantage of the GUB is that it provides data structures and functions to maintain large sets of graphs. For some of the matching algorithms it implements this is in fact necessary since they rely on especially preprocessed models. But the other algorithms, too, make use of this database facility and we did not have to care about graph maintenance ourselves. However, in order to facilitate its usability we implemented a visual front end that, as sown in Fig. 4.5(a), allows to view, save, and load the content of a database of model graphs. It also allows to switch between the algorithms provided by the GUB and furthermore enables to set costs and other parameters that enter into the calculation of isomorphisms.

Given a set of models and an input graph, the GUB determines error-correcting subgraph isomorphisms based on costs of the edit-operations vertex deletion, edge deletion and insertion and label substitution. As explained above, the costs of these operation must be provided manually and their choice usually depends on the intended application. For our purposes, we set the costs of the first three operations to 1, 1, 10, respectively. This choice has stood the test of practice and generally yields matching results of good quality. The considerable high costs for edge insertion are due to the observation that the GUB tends to insert edges to minimize subgraph distances. However, when dealing with mating feature graphs, practice has shown that edge insertion introduces relations among features that do not exist in reality and thus results in useless matches. In gen- 


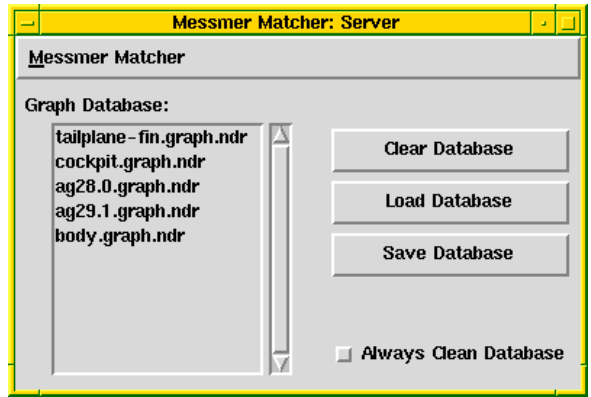

(a)

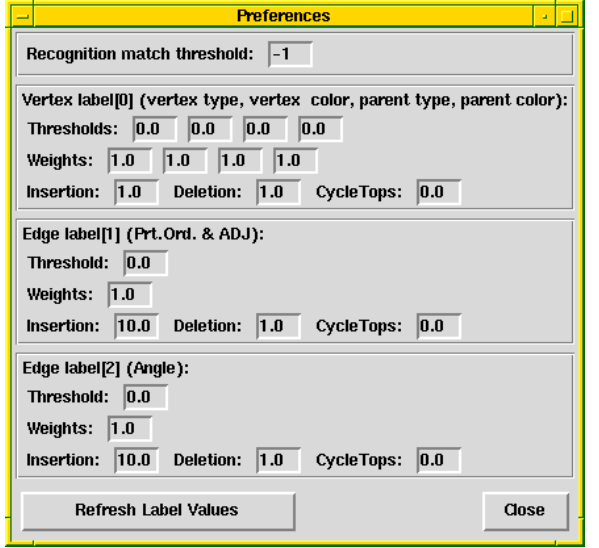

(b)

Figure 4.5.: Screenshots of a graphical user interface that facilitates the handling of the GUB. 4.5(a) A window that displays the current content of a database of graphs. 4.5(b) A window to set costs and weights for graph matching with the GUB.

eral, it is better not to detect any isomorphism than to match an input graph with a model that was transformed into an infeasible mating feature graph.

Costs $C_{S}$ for label substitutions are calculated from the weighted distances of label values, i.e.

$$
C_{S}=\sum_{i=1}^{n} w_{i} d\left(l_{1_{i}}, l_{2_{i}}\right)
$$

where $n$ is the dimension of a label value (for instance, for vertex labels of a mating feature graph, we will have $n=3$ since $l_{1}, l_{2} \in T \times O \times C$ (see page 78)). And as the labels assigned to mating feature graphs are of symbolic nature we use the following metric to compute their distance

$$
d\left(l_{i_{1}}, l_{i_{2}}\right)= \begin{cases}0 & \text { if } l_{i_{1}}=l_{i_{2}} \\ 1 & \text { otherwise. }\end{cases}
$$

Finally, simply setting all the weights $w_{i}$ to 1.0 proved to be a good choice for most assembly recognition tasks.

\section{Different Recognition Tasks}

Table 4.1 summarizes some typical results that can be accomplished by means of mating feature graph matching. It shows matches visualized using LEDA [81]. Each graph in 


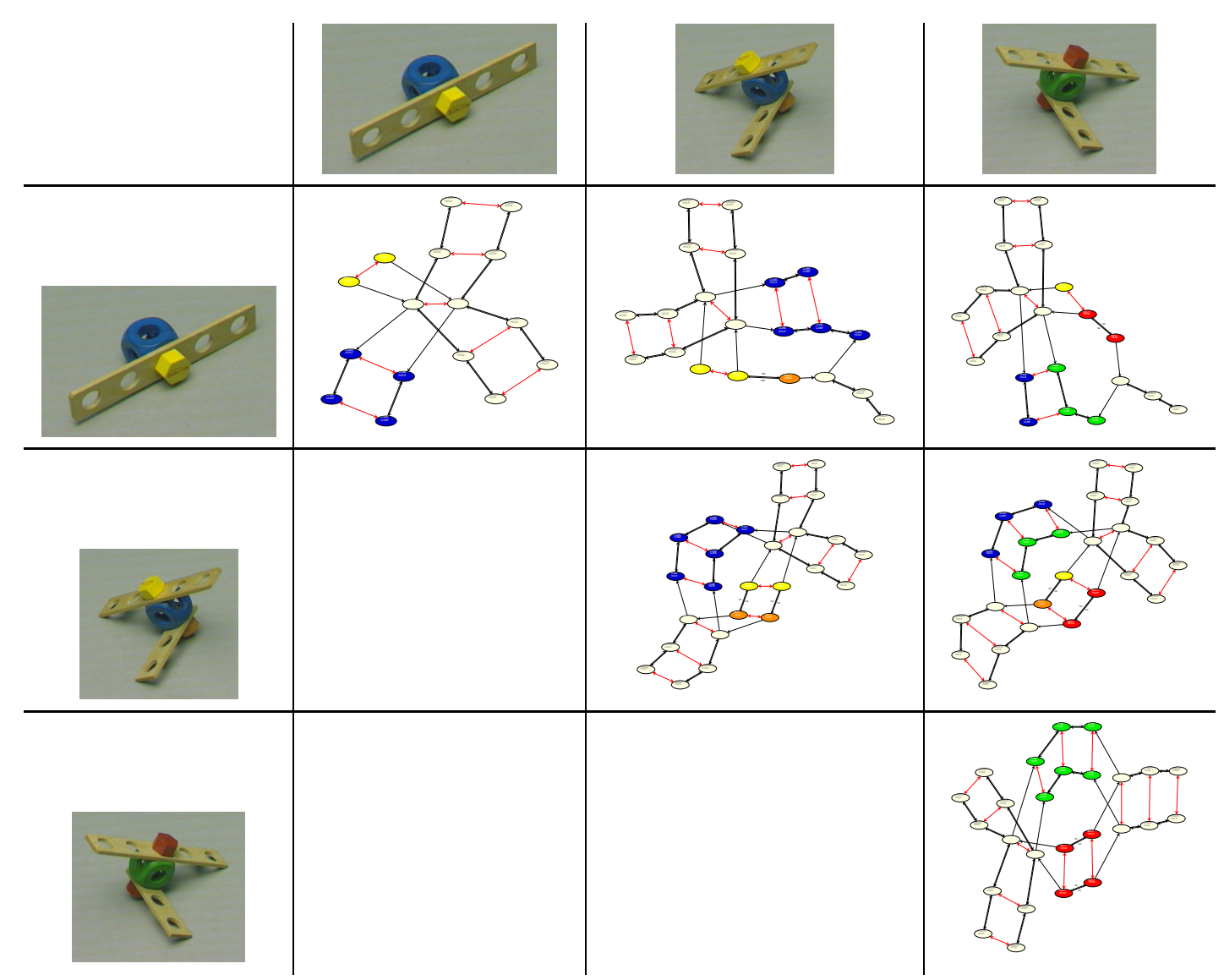

Table 4.1.: Examples for some of the matching capabilities of the GUB: it allows to recognize that two graphs represent the same assembly, that one represents a subassembly of the other, or that they represent similar assemblies.

the table actually consists of two mating feature graphs connected via edges colored in red indicating how vertices of a model graph were matched with those of an input graph. Edges of black color reflect the familiar topological relations within the assembly represented by the corresponding subgraph; their labels were omitted for the sake of readability.

The diagonal elements of the table display examples of exact matches between two mating feature graphs. Hence, it is possible to recognize that two assemblies are made from the same parts and show the same mating relations, i.e. that they are topologically identical. As the GUB enables the detection of subgraph isomorphisms it is also possible to recognize part of relations among complex objects. The second entry of the first row displays an exact subgraph isomorphism and thus constitutes an example of subassembly recognition. 


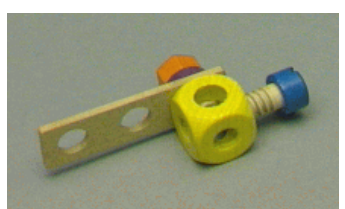

(a)

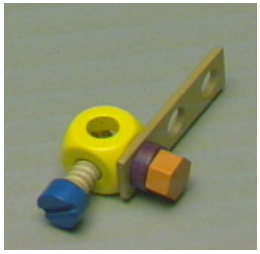

(b)

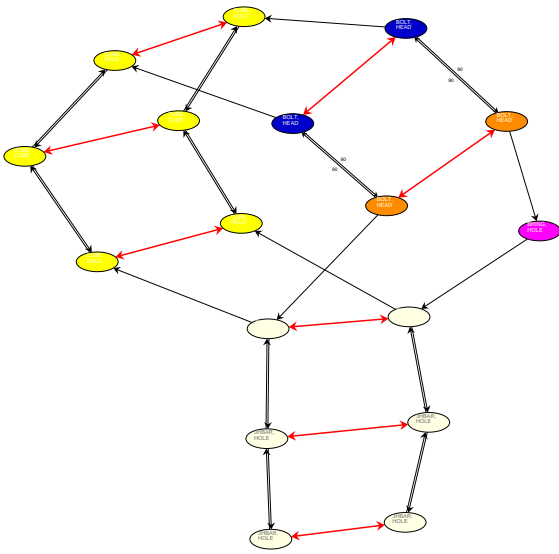

(c)

Figure 4.6.: Example of an error-correcting subgraph isomorphism. 4.6(a) An assembly known from Fig. 3.21 on page 56. 4.6(b) The same assembly seen from a different perspective so that the purple ring was recognizable. 4.6(c) The error-correcting isomorphism between the two mating features graphs generated from the different views.

Psycholinguistic experiments that are currently being conducted within the SFB 360 indicate that if humans assign a name to a baufix ${ }^{\circledR}$ assembly, they believe to recognize a real world object. This cognitive process first of all is triggered by the form of the assembly while the colors of its parts seem to be irrelevant. Thus, there should be no reason to treat the two complex assemblies shown in the table as different objects. They consist of objects of the same type and they are topologically and even geometrically identical. They only differ in the color of the engaged objects. Fortunately, as the third element in the second row and the third element in the first row show, the GUB's capability to detect error-correcting subgraph isomorphisms allows to cope with varying object color in assembly recognition. If for any reason it is not desired to obtain matches like this, they could be prevented by increasing the corresponding weights in the computation of costs for label substitutions.

Furthermore, error-correction by means of vertex or edge deletion or insertion allows to recognize similar assemblies or to recognize assemblies in spite of perspective occlusion. An example of the latter is shown in Fig. 4.6. Figure 4.6(a) displays an assembly already known from Fig. 3.21 on page 56. There, we discussed how to incorporate action detection into assembly detection in order to cope with perspective occlusions. Caused by the perspective, the purple ring in the assembly is hardly visible in Fig. 4.6(a). Therefore, 


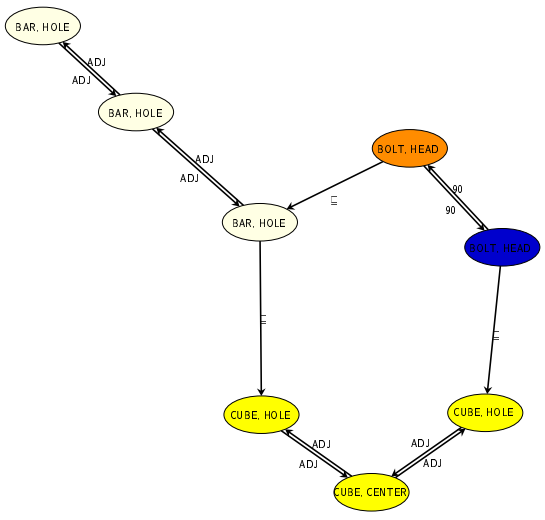

(a) MFG from Fig. 4.6(a)

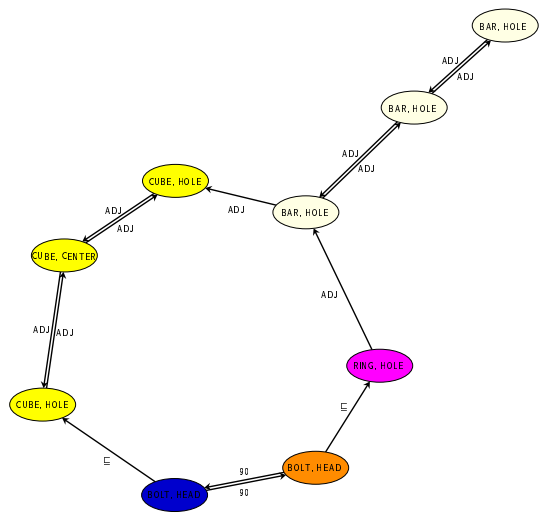

(b) MFG from Fig. 4.6(b)

Figure 4.7.: The mating feature graphs whose match is shown in Fig. 4.6.

it cannot be recognized and assembly detection without information about observed assembly tasks will yield a structure that does not contain the ring. Neither does of course the mating feature graph derived from the corresponding assembly sequence plan. In Fig. 4.6(b), however, the ring is visible and the mating feature graph derivable from this image is correct. Figure 4.6(c) shows how both graphs were mapped onto each other.

Actually, with the costs of edit operations chosen as explained above and the incorrect mating feature graph given as a model the subgraph distance between the two graphs only amounts to 1 . The reason becomes obvious if we consider Fig. 4.7 which shows both graphs in detail. Obviously, the simplest sequence of edit operations that transforms the graph in Fig. 4.7(a) into a subgraph of the one in Fig, 4.7(a) is the deletion of the 巨-edge from the orange bolt head to the hole of the bar. Edge deletion, however, is cheap.

Again, if one is not interested in matches like this, the costs or weights of the corresponding edit operations must be modified accordingly. The choice of adequate costs in fact remains a very interesting issue for further research. Currently, there are plans for psychological experiments that should help to learn more about the way humans perceive (baufix ${ }^{\circledR}$ ) assemblies. For instance, it will be interesting to determine up to which degree of deviation they accept different assemblies to represent the same object. From the results we hope to develop more sophisticated or cognition based costs of edit operations for graph isomorphisms and thus to proceed towards human like performance in assembly recognition.

\section{An Autonomously Learning Vision System}

According to what was said so far, assembly recognition is a two step procedure. In the first step clusters of labeled image regions are analyzed syntactically in order to detect assembly structures in an image. This step is based on a simple and compact context 
free grammatical model of the general component structure of composite objects. Like one would expect from a grammatical approach to pattern recognition, this model allows to detect a huge variety of structures based on a small set of productions and does not require knowledge of individual assemblies. Therefore, though the modeling effort is low, the number of structures that can be treated is nearly unrestricted.

Syntactic analysis according to the assembly grammar can be performed by a semantic network. This allows to register how the objects of a cluster a situated to each other and thus enables to determine mating relations among assembly parts from image analysis. This information can be translated into a graph based representation that detailedly captures assembly topology. I.e. comprehensive topological models of individual assemblies can be derived automatically and do not have to be provided by an expert.

After deriving a mating feature graph from a plan it can be matched against a database of previously calculated graphs. If no match is possible, the graph is assumed to represent a yet unknown assembly and is inserted into the database of models. This, in fact, realizes a learning vision system that autonomously extends its knowledge base.

By now, there is no mechanism to automatically assign symbolic information to the mating feature graphs contained in a knowledge base. If a modeled assembly depicts a real world object like a tailplane-fin or a locomotive, a corresponding label must be manually annotated to the graphs. But as the SFB 360 is dedicated to cooperative multi-modal man-machine dialog and interaction, solutions to this problem are foreshadowing. Just recently, there has been considerable progress in integrated speech and vision processing [133] as well as in system architecture for intelligent man-machine interaction [7]. Thus, concluding from the present achievements we presume that it will soon be possible to automatically ground and learn the meaning of a mating feature graph during a sequence of cooperative assembly tasks.

\subsubsection{Assembly Reconstruction}

A problem we did not mention yet is exemplified in Fig. 4.8. The figure demonstrates that topologically equivalent assemblies may differ geometrically. This dilemma is well known in assembly modeling and is marvelously expressed in a definition by Hurt [59]:

Geometry is where the objects are located; topology is why they are located there ${ }^{6}$.

This indeed agrees with what we said before: possible assembly topologies are predetermined by rules of construction. These specify how mating features can be attached to one another but need not completely constrain the mutual position of interconnected objects. There rather might remain orientational degrees of freedom. In the baufix ${ }^{\circledR}$ domain, for instance, a bar may rotate about a bolt.

\footnotetext{
${ }^{6}$ Found in [100].
} 


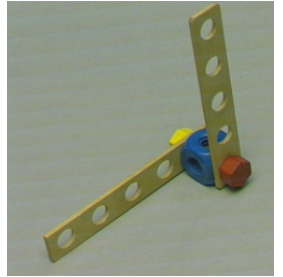

(a)

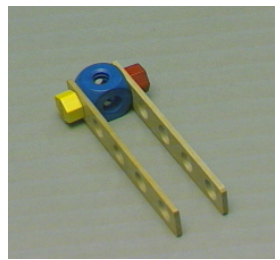

(b)

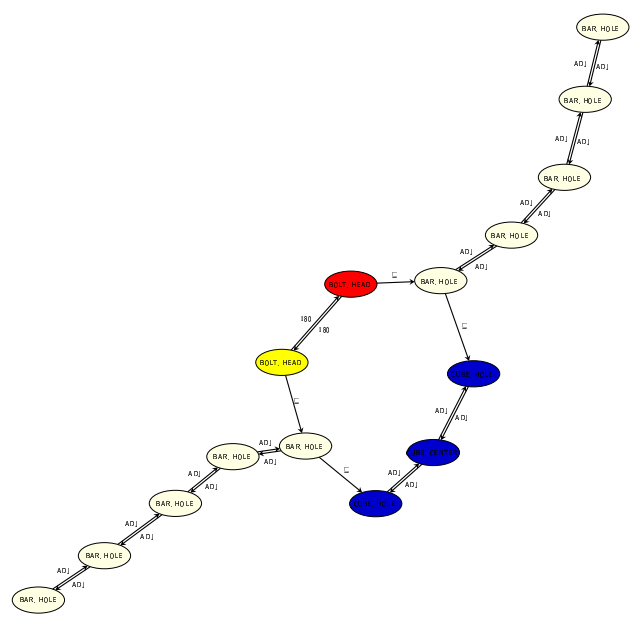

(c)

Figure 4.8.: An example of two geometrically different assemblies of the same topology.

The assemblies in Figs. 4.8(a) and 4.8(b) obviously consist of exactly the same parts and show the same attachments. Thus, both correspond to the mating feature graph shown in Fig. 4.8(c) and assembly recognition based on matching feature graphs will not be able to distinguish them. A distinction would require knowledge of three-dimensional assembly structures which is not available from the techniques we discussed so far. Moreover, geometric information like this will generally not be obtainable from a single image but will at least demand stereo vision.

If a scene is observed by a pair of cameras whose optical parameters and mutual location and orientation are known or may be estimated, the spatial position of entities visible in both image signals can be determined (cf. [34]). Mathematically, depth estimation is not too demanding a task. Calculating the spatial position of an entity is not difficult, the problem is to provide the data the calculation relies on. In its most simple form, this data is given as a set of pairs of image coordinates $\left(\boldsymbol{x}, \boldsymbol{x}^{\prime}\right)$ where $\boldsymbol{x}$ is the coordinate of a pixel in the one and $\boldsymbol{x}^{\prime}$ is the coordinate of a pixel in the other image and both depict the same aspect of the scene. Thus, if the content of two images of a scene should be reconstructed three-dimensionally, the signals must be searched for corresponding features. And this has proven to be a challenging problem.

\section{Stereo Correspondences from Graph Matching}

Usually, correspondences are searched for in the raw or slightly preprocessed image data and a large variety of methods to do so is known (cf. [34, 120]). Our situation, 
however, is different for we are dealing with symbolic information. The data we use for assembly recognition are labeled image regions depicting elementary objects of the baufix ${ }^{\circledR}$ construction-kit. As those regions are characterized by a single color and a description of their boundaries instead of a collection of individually colored pixels, no backward inference to the original signal is possible and the usual methods to find correspondences cannot be applied.

But in Chapter 3.3.1 we explained how to compute image coordinates of mating features of objects comprised in an assembly. In this chapter, we introduced the idea to model assemblies by means of graphs representing relations among mating features and to match those graphs to accomplish recognition. Thus, there is an obvious approach to finding pairs of corresponding image coordinates from symbolic information. We just have to extend the vertex labels of mating feature graphs such that they not only characterize a feature's type and the type and color of the object it belongs to but also its image coordinates. Instead of the vertex labeling function $\mu$ presented on page 78 we henceforth will consider a function $\mu^{\prime}$ with

$$
\mu^{\prime}: L_{V} \rightarrow T \times O \times C \times \mathbb{R}^{2} .
$$

This extension of course requires to integrate feature coordinates into the high-level assembly plans where mating feature graphs are derived from. And we should verify if this will violate the relation to semantics theory we found earlier. Fortunately, the former is not a problem and the latter is not the case. Feature coordinates are available from image analysis by means of an ERNEST network and can be introduced into the plan generating grammar such that it remains context free. We simply introduce a new variable and production

$$
\text { coord = "("num "," num")" }
$$

and replace productions like for instance

$$
\text { holes =( "()"| "(BOLT" num ")") }\{\text { holes }\}
$$

by productions that additionally derive the new variable:

$$
\text { holes }=(\text { "()"| "(BOLT" num ")") coord }\{\text { holes }\} .
$$

As the necessary modification of the semantics function is straightforward, we will not discuss it further.

Thus, given a graph representation where vertices also describe the image coordinate of a feature, graphs derived from stereo images as shown in Fig. 4.9 can be matched in order to obtain pairs of corresponding coordinates. However, we must regard that corresponding features are from different images so their image coordinates will hardly 

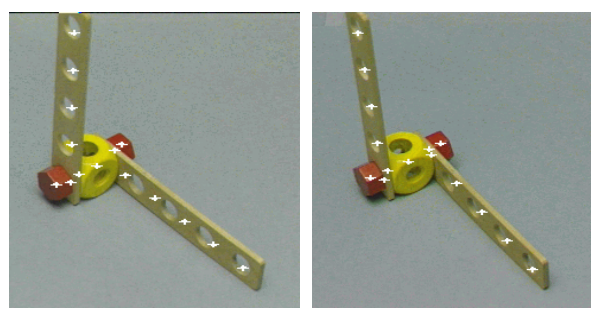

Figure 4.9.: Stereo images of an assembly with calculated positions of mating features cast into them.

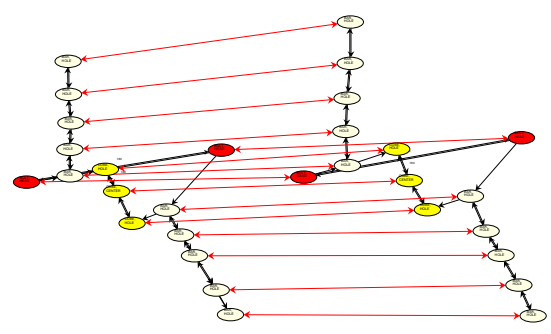

(a)

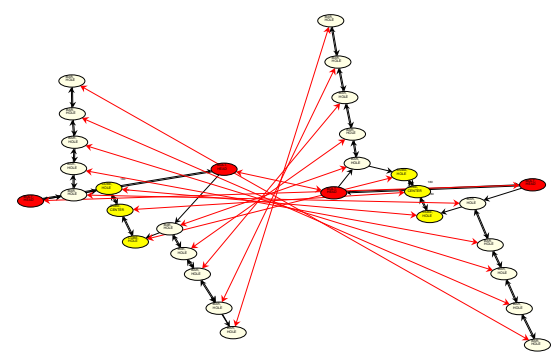

(b)

Figure 4.10.: Two possible isomorphisms between the mating feature graphs derived from the images in Fig. 4.9. In both cases the subgraph distance vanishes.

match. If not cared for, this will cause the GUB to perform numerous label substitutions while searching for a subgraph isomorphism. Remembering that the costs of label substitutions are are given by

$$
C_{S}=\sum_{i=1}^{n} w_{i} d\left(l_{1_{i}}, l_{2_{i}}\right)
$$

we can filter out coordinates from the computation of isomorphisms by just setting their weights to 0.0 .

\section{Finding the Right Match}

Another issue to care about is visualized in Fig. 4.10: if an assembly is axially symmetric in its parts and attachments, it will have a symmetric mating feature graph $\Gamma$. As a consequence, there will be several automorphisms, i.e. isomorphisms from $\Gamma$ to itself which do not require any edit operations. Thus, they do not raise costs and the GUB will not be able to rank them. 
From a topological point of view the assembly shown in Fig. 4.9 is axially symmetric and its mating feature graph has two automorphisms which are shown in Fig. 4.10. If we were dealing with assembly recognition as presented in the previous section, this would not matter because detecting any match of low subgraph distance would accomplish the task. Here, however, we are interested in 3D reconstruction based on corresponding image coordinates. And since the automorphism in Fig. 4.10(b) will produce pairs of incongruent coordinates, only the one in Fig. 4.10(a) is acceptable.

Of course it would be desirable if there was a mechanism to automatically decide which out of several sets of matched coordinates should be used for depth estimation. And indeed, assuming two pinhole cameras in a conventional stereo geometry where the optical axes are parallel and on the same height and the focal length is identical, a simple heuristic can support the decision. Given two corresponding pixels $\boldsymbol{x}_{l}=\left(x_{l}, y_{l}\right)$ and $\boldsymbol{x}_{r}=\left(x_{r}, y_{r}\right)$ in the left and right image, respectively, their disparity is defined as

$$
d=x_{r}-x_{l} .
$$

With $f$ denoting the focal length of the cameras and $b$ the horizontal distance between their optical centers, the depth $z_{w}$ of the real world point $\boldsymbol{x}_{w}$ depicted by $\boldsymbol{x}_{l}$ and $\boldsymbol{x}_{r}$ results from the identity:

$$
d=\frac{f}{z_{w}} b \Leftrightarrow z_{w}=\frac{f}{d} b .
$$

Thus, if two points $\boldsymbol{x}_{w}$ and $\boldsymbol{x}_{w}^{\prime}$ have approximately the same depth $z_{w} \approx z_{w}^{\prime}$ the corresponding disparities will be approximately equal, too. This justifies the following approach. As the spatial extension of baufix ${ }^{\circledR}$ assemblies is rather small, all their mating features will have approximately the same distance to a pair of stereo cameras. Moreover, as indicated in Fig. 4.10(a), stereo imagery basically means a constant horizontal translation of coordinates. Therefore, given a set of $N$ corresponding feature coordinates $\left(\boldsymbol{x}_{l_{i}}, \boldsymbol{x}_{r_{i}}\right)$ the disparities or more general the distances

$$
d_{i}=\left\|\boldsymbol{x}_{l_{i}}-\boldsymbol{x}_{r_{i}}\right\|
$$

should be more or less the same. From Fig. 4.10(b), in contrast, we can deduce that pairs of incongruent coordinates will accord with a reflection at a center of symmetry. Therefore, the distance between two wrongly associated pixels will depend on their distance to the center. Hence, if we compute the mean distance $\mu=\frac{1}{N} \sum_{i=1}^{N} d_{i}$ among a set of corresponding coordinates, the standard derivation

$$
\sigma=\sqrt{\frac{1}{N} \sum_{i=1}^{N}\left(d_{i}-\mu\right)^{2}}
$$

should be small for the acceptable automorphism of a mating feature graph and higher for an unacceptable one. 


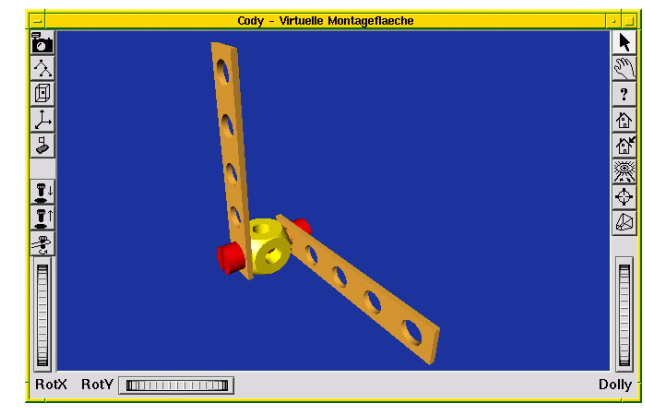

Figure 4.11.: Virtual reconstruction of the assembly in Fig. 4.9.

Of course, there are no pinhole cameras in our experiments. Yet practical experience has proven the suitability of this heuristic. For the automorphism in Fig. 4.10(a), for instance, a standard derivation $\sigma=16.75$ was computed while the one in Fig. 4.10(b) yielded $\sigma=159.56$.

\section{Reconstruction Accuracy}

It must be mentioned that the pixel coordinates of mating features used for 3D reconstruction are of minor accuracy. As explained in Chapter 3.3.1, they are coarsely estimated during syntactic analysis. In Fig. 4.9, for instance, we can see that most of the calculated positions of the bar holes not coincide with the center of the holes. Yet their degree of accuracy is sufficient to determine details of object attachments. But it certainly will never yield precise spatial positions of mating features. The coordinates resulting from depth estimations must rather be considered as rough estimates of the actual spatial locations of mating features.

Nevertheless, the rough estimates are sufficiently accurate to create virtual prototypes of visually analyzed assemblies. Figure. 4.11 displays the 3D model that was derived from the images in Fig. 4.9. It was created using a CAD tool for virtual construction which was developed within the SFB 360 [64].

\subsection{Summary}

Syntactic approaches to pattern recognition are well suited to describe and detect patterns of interrelated primitives. However, the more complex a pattern is the more syntactic descriptions it will generally have. Mechanical assemblies are three-dimensional patterns composed of elementary objects. In Chapter 3, we saw that context free grammars can derive component structures of assemblies and may guide visual assembly detection. In this chapter, we took a step ahead and considered the problem of visual assembly recognition. 
Our first concern was whether syntactic recognition would be feasible or be affected by the possible multitude of descriptions. Thus, we empirically studied the complexity of syntactic modeling of baufix ${ }^{\circledR}$ assemblies. Based on a simple modeling scheme that represents a bolted assembly as a set of partial orders among its parts we calculated AND/OR-graphs of several assemblies of different topology. Counting the number of trees in the graphs revealed that the average number of syntactic structures grows exponentially with the number of bolts contained in an assembly.

This finding has an impact on the choice of a suitable method for assembly recognition. Simple syntax based approaches seem infeasible because robust and reliable syntactic recognition of individual assemblies would require to store too many descriptions or grammars.

A solution to this problem resulted from considering what kind of information is available after assembly detection. In Chapter 3.3.1, we explained that assembly detection by means of an ERNEST network yields high-level sequence plans which encode topological relations among interconnected parts. Here, it was pointed out that these relations can be modeled using a special type of labeled graphs which we called mating feature graphs. As their vertices represent mating features of elementary objects and their edges indicate topological relations among those, mating feature graphs can be seen as a refinement of liaison graphs which represent adjacency relations among assembled objects [17, 28, 51].

Mating feature graphs are topologically unique assembly models. We explained that syntactically different plans of a given assembly all lead to the same graph and discovered another correspondence between the worlds of assembly modeling and formal language theory: syntactic assembly structures and high-level assembly sequence plans correspond to words of context free languages. Mating feature graphs form a semantic domain and can be understood as the denotational semantics of assembly plans.

Based on mating feature graphs assembly recognition becomes a problem of graph matching. As graph matching itself is a nontrivial problem, we gladly applied the graph matching toolkit of the University of Berne (GUB) which provides efficient algorithms to detect error-correcting subgraph isomorphisms between a database of model graphs and an input graph $[22,82,83]$. Given a set of suitable costs for the edit operations performed by the GUB, we presented examples of assembly and subassembly recognition by calculating exact graph or subgraph isomorphisms. Further examples demonstrated that searching for error-correcting subgraph isomorphisms between mating feature graphs also copes with perspective occlusions or can recognize similar assemblies.

Brought together, the methodologies presented so far constitute an autonomously learning vision system. Based on the simple grammar of baufix ${ }^{\circledR}$ assemblies a huge variety of assembly structures can be found from image parsing. The resulting plans are automatically translated into mating feature graphs with can be matched against a labeled set of known graphs. If the system cannot find any (cheap) isomorphism the input will be inserted into the database. Coupled with software modules for multi-modal man- 
machine interaction (cf. [7]) the system is able to acquire assembly knowledge far beyond the predefined syntactic productions.

Despite their suitableness for many recognition tasks there remains a shortcoming of mating feature graphs. They do not represent details of assembly geometry. Therefore, based on mating feature graphs it is impossible to distinguish topologically identical assemblies of different geometry. However, derived from stereo image data mating feature graphs may serve as a source of information for 3D object reconstruction. If image coordinates are assigned to their vertices, isomorphisms between feature graphs will yield pairs of corresponding pixels so that spatial positions of mating features can be calculated. Since the coordinates used for their computation are of minor accuracy, these $3 \mathrm{D}$ positions are rather coarse approximations. Nevertheless, they are precise enough so that a CAD tool for virtual construction can be used to reconstruct the three-dimensional appearance of the corresponding assembly.

\subsection{Intermediate Conclusion}

Before continuing with the next chapter we shall briefly revisit issues raised in the introduction and reflect what we can say about them now.

Page 5 in the introduction listed a couple of aspects that have to be regarded when investigating principles and mechanisms behind cooperative interaction in flexible assembly. In a scenario like studied in the SFB 360, mechanical assemblies pose problems of planning, learning, and recognition. They are entities that have a structure resulting from a process and may also have semantic and pragmatic aspects.

The framework of combined syntactical and relational assembly modeling introduced in Chapters 3 and 4 can treat all these facets. Syntactic descriptions and graph based models both provide a means to describe the structure of assembled objects. While hierarchical syntactic assembly models implicitly encode construction processes or plans, graph based models explicitly denote where this processes lead to.

Assigning names to graphs stored in an automatically generated database enables to seize the semantics or depictional metonymy of composite objects. With respect to the introductory example on page 1 it is, for instance, possible to label a graph model of an assembly as a steam engine after it was generated from image analysis. Therefore, knowledge of individual assemblies can be learned.

By means of graph matching assemblies can be recognized and searching for subgraph isomorphisms also allows to treat pragmatic aspects. During construction, for example, the mating feature graph of an assembly representing the rear of a steam engine may be named respectively. Though this approach will enable the use of specific instructions, at least in this example, it would be of poor cognitive adequacy because it would reverse the way humans introduce names for complex objects. Names of subassemblies are usually introduced after the whole assembly was recognized as a model of a real world object. 
Therefore, another idea is to extend the vertex labeling scheme of mating feature graphs such that each vertex also encodes information of the intrinsic spatial position of the corresponding feature in the assembly. In this way an artificial communicator could automatically locate the rear of the steam engine if required by its instructor. However, a mechanism that assigns spatial identifiers to vertices of mating feature graphs is not yet realized but remains a promising topic for future work.

Considering all this, the undisputed quintessence of what we have discussed so far is that structural approaches treat numerous phenomena in cooperative assembly. In combination, syntactic and graph based methods provide a suitable framework to describe and model different aspects of assemblies. In particular, they provide an avenue to flexible assembly recognition from vision. 


\section{Temporal Context}

The previous chapters presented the benefits of structural approaches to visual assembly recognition. However, syntactic image analysis by means of a semantic network, the generation of a mating feature graph, and the search for a corresponding one may be rather time consuming if the assemblies that are concerned are large.

Yet in the introduction we pointed out that we are dealing with a cooperative construction scenario where complex assemblies do not appear instantaneously but are build in a step by step procedure. Moreover, a usual assembly task in this scenario does not affect all objects in the construction cell. Rather, in each step of an assembly process only a few objects are manipulated while most remain unmoved. And again, sometimes (complex) objects are just rearranged but not attached to others. In a situation like this, the structure of assemblies does not change and their visual appearance will only vary slightly.

A pragmatic approach that copes with the first phenomenon was reported in [7]. Integrated into a system for intelligent multi-modal man-machine communication in assembly, assembly detection and recognition do not have to be performed at every instant in time. Provided a module that registers changes in the assembly cell, the algorithms presented so far are activated only if a new cluster of objects appears in the scene. However, even slight rearrangements of an assembly usually will be interpreted as the appearance of a new cluster so that this approach only accelerates the treatment of immobile assemblies.

Another idea was to reuse assembly knowledge that had been derived earlier in a process. Actually, in prior contributions resulting from our research project Kummert et al. $[72,73]$ already proposed information propagation through time to speed up semantic network based recognition of elementary objects. Their idea was to verify if object instances generated for a previous image of a sequence could be correlated with the current signal. If so, instances are kept and exhaustive computations must only be performed for those parts of an image that considerably differ from its predecessor. However, due to the complex internal structure of assemblies this approach proved to be hardly applicable to assembled objects: to assure correctness all part links in an instance tree of an assembly must be verified so that there will be no substantial speed-up.

This motivated the investigation of completely different approaches to assembly recognition [10]. In this chapter, we will discuss methods that, though structural, are not based on grammatical or relational modeling. Nevertheless, we will not discard the 


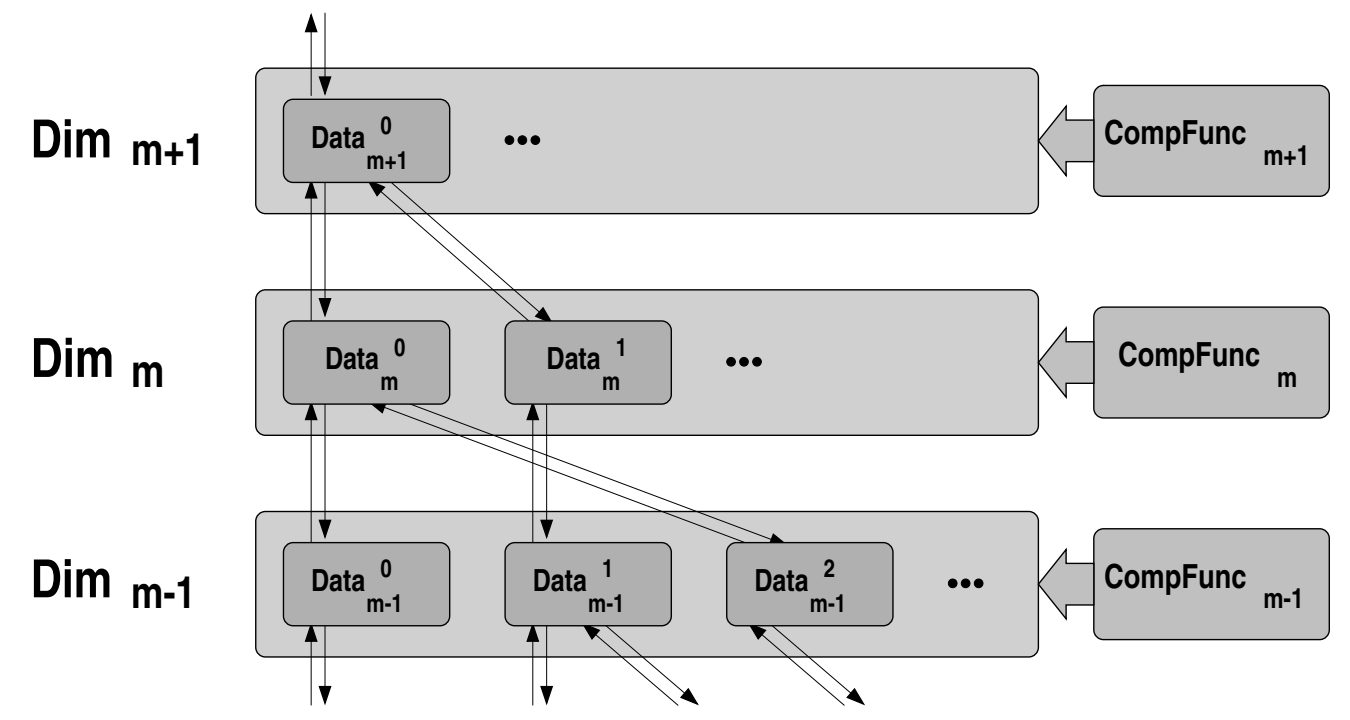

Figure 5.1.: Concept of a hierarchical visual memory to store and relate information for assembly recognition. Data from different stages of processing is stored in different tables $\operatorname{Dim}_{m}, m \in \mathbb{N}$. Each table is associated with a function CompFunc $_{m}$ that compares the corresponding datasets. Data that represents the same assembly but appears in different tables is related via pointers.

techniques developed so far but rather extend them by a step of preprocessing. The system presented next will make use of different global assembly features that can be gathered during syntactic analysis. It stores, organizes, and interrelates different types of data in order to reuse information known from earlier stages of a process. As we will see, its architecture is flexible, extendible, and allows to integrate various pattern recognition paradigms. I.e. we will present a hybrid approach to assembly recognition that accumulates information throughout an assembly process and thereby speeds up performance.

\subsection{Concept of a Visual Memory}

Up to now, we have seen various examples of clusters of labeled image regions and of image coordinates of mating features which we used to discuss different aspects of assembly detection and recognition. But, revisited, they also suggest that the appearance of a cluster of regions or the $2 \mathrm{D}$ distribution of mating features are visual characteristics of individual assemblies and might be used for recognition.

This leads to the idea of a database-like visual memory that stores information from different steps of image processing and associates it with symbolic assembly knowledge. 
Its general conceptual layout is sketched in Fig. 5.1. The memory consists of several tables, called dimensions, each containing a specific kind of data. Since data in the higher levels is computed from lower level information, the dimensions are implicitly organized in a hierarchy. For each dimension there is a specialized function that compares the entities contained in that dimension. Here, this memory concept differs from classical databases: since visual assembly recognition is a pattern recognition task, data which is to be stored in the memory might be similar to but usually not identical with earlier stored one. Thus, we need sophisticated functions computing similarities instead of key comparisons known from database theory. Pieces of information from different dimensions which represent the same assembly are connected via pointers. Therefore, it will be possible to retrieve information concerning an assembly given just a certain aspect. Note that the comparison functions can be chosen freely. Hence, the concept sketched in Fig. 5.1 is modular and facilitates the exchange of pattern recognition techniques.

Given an input image, assembly recognition based on this concept should be accomplished by the following procedure:

1. If there is yet no data in the memory, carry out a conventional syntactic assembly detection and store the results from intermediate steps of processing, i.e. from different levels of abstraction, in the corresponding levels of the memory.

2. If there already is data in the memory, compute the lower most type of information from the current image and check whether similar information is contained in the lowest memory dimension. If this is the case, trace the pointers to the highest dimension, report the information found there and stop. If there is no similar data in the lowest dimension, register it and continue with 3.

3. Continue image processing. If similar data can be found in the corresponding hierarchical level, interconnect this entity and the recently registered data from the previous level, trace the pointers to the highest dimension, and stop. If similar information has not yet been stored in the current dimension, store it and draw pointers to the corresponding data in the level below.

Iterate this step until the last level of processing is reached.

In order to test this idea, we implemented an experimental memory of only a few dimensions. Please note that the corresponding pattern recognition methods which we will briefly present in the following may not represent the state of the art in the respective field. Rather, they are meant to verify the practical applicability of the underlying layout.

\subsection{An Experimental Implementation}

Our current implementation of the memory realizes four dimensions. The lower most one stores clusters of labeled regions like shown in Fig. 5.2(b) which represent the objects 


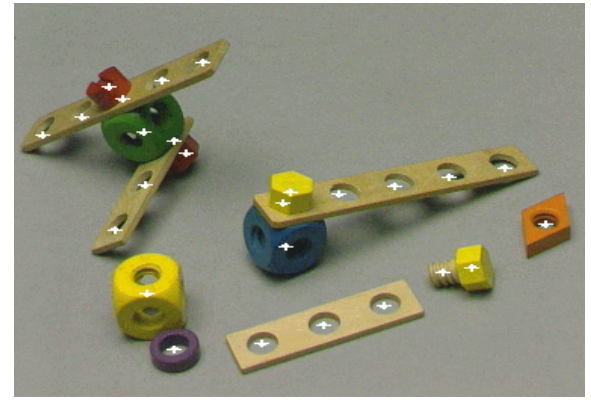

(a)

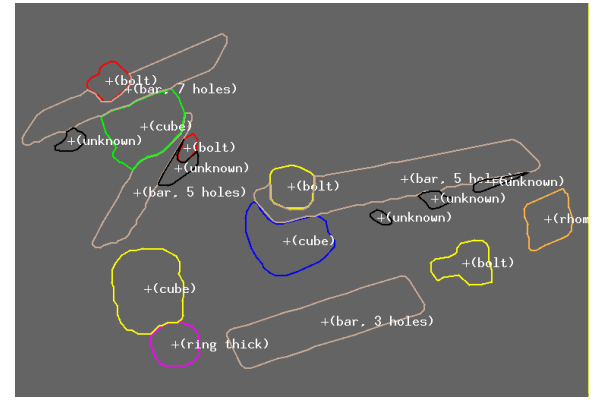

(b)

Figure 5.2.: A scene of baufix ${ }^{\circledR}$ objects and the corresponding object recognition results. The mating feature coordinates cast into the image on the left were calculated before assembly detection took place.

comprised in an assembly. Together with the corresponding comparison function, it can thus be interpreted as a region or shape based recognition module.

The next dimension contains sets of those mating feature coordinates that can be extracted directly from object regions. For abbreviation, we will call them interest points henceforth. As explained on page 52 , in the baufix ${ }^{\circledR}$ domain one can apply heuristics such that interest points can be found for almost every feature. The holes of a bar, for instance, are assumed to be equally distributed along the principle axis of the corresponding image region. An exception are the holes of cubes whose positions cannot be calculated until mating relations have been found by syntactic cluster analysis. As we can see from Fig. 5.2(a), the distribution of interest points resembles the skeleton of objects or clusters of objects.

The data in the third hierarchical level describes syntactic assembly structures resulting from object cluster parsing. And, finally, the highest level stores names of assemblies which are not generated automatically but must be assigned interactively.

While the comparison of names is trivial, the last chapter discussed that comparing syntactic assembly structures is rather impossible and, in fact, in the experimental implementation introduced here, assembly tree comparison was postponed. But for clusters of labeled regions and sets of interest points one can again think of a variety of methods to compute resemblance. To compare two region clusters in the memory each cluster is characterized by a four-dimensional feature vector that represents the number and types of objects comprised in the cluster and the compactness and exentricity of the merged regions it consists of. Given two feature vectors $\boldsymbol{v}$ and $\boldsymbol{v}^{\prime}$, a function $s$ defined by the boolean expression

$$
s\left(\boldsymbol{v}, \boldsymbol{v}^{\prime}\right)=\bigwedge_{i=1}^{4}\left(\left|v_{i}-v_{i}^{\prime}\right| \leq 0.3 \max \left(v_{i}, v_{i}^{\prime}\right)\right)
$$


yields whether the vectors represent the same cluster. I.e. two clusters are said to be equal if for each pair of corresponding features the difference does not exceed $30 \%$ of the larger value. Of course, this is a crude approach. But it is fast and well suited to recognize assemblies that neither change their position nor their orientation throughout a sequence of construction tasks.

\subsubsection{Matching Interest Points}

More effort was spent in matching interest points. Besides its 2D image coordinates each interest point is assigned the type of mating feature it represents. Thus, an interest point is a tuple $p=(\boldsymbol{x}, t)$ with $\boldsymbol{x} \in \mathbb{R}^{2}$ and $t \in T=\{$ BOLT-HEAD, BAR-HOLE, CUBE-HOLE, $\ldots\}$. Apparently, given different images of an assembly the sets of interest points derivable therefrom will differ. Corresponding interest points will have different coordinates and due to perspective occlusion or recognition errors it might happen that there are points in either set which do not have a counterpart in the other set. Therefore, flexible techniques to estimate the similarity of sets of interest points are needed.

By defining a distance $d\left(p, p^{\prime}\right)=\left\|\boldsymbol{x}-\boldsymbol{x}^{\prime}\right\|+d_{T}\left(t, t^{\prime}\right)$ between interest points where we choose

$$
d_{T}\left(t, t^{\prime}\right)= \begin{cases}0 & , \text { if } t=t^{\prime} \\ \frac{\sqrt{2}}{2} & , \text { otherwise }\end{cases}
$$

the set $\mathbb{R}^{2} \times T$ becomes a metric space and methods to measure distances or similarities between sets of interest points are available. Out of the many known approaches to point set matching (cf. e.g. [38, 79] and the references therein) we chose two fast ones for our scenario: calculating the Haussdorff distance between two sets and computing an affine mapping between sets derived by means of a gradient descent method.

\section{A Gradient Descent Method}

Our gradient descent approach determines the similarity of two point sets $P_{0}$ and $P_{1}$ by looking for an affine transformation $\boldsymbol{A}$ that most accurately maps the spatial coordinates of the points in $P_{1}$ onto coordinates of points in $P_{0}$.

This is done as follows: First, the spatial coordinates $\boldsymbol{x}$ of all points in both sets are normalized to the unit square. Then, two points $p_{1}^{0}=\left(\boldsymbol{x}_{1}^{0}, t_{1}^{0}\right)$ and $p_{2}^{0}=\left(\boldsymbol{x}_{2}^{0}, t_{2}^{0}\right)$ are chosen from $P_{0}$ such that their spatial distance in maximal in $P_{0}$, i.e.

$$
\left\|\boldsymbol{x}_{1}^{0}-\boldsymbol{x}_{2}^{0}\right\|=\max _{p, p^{\prime} \in P_{0}}\left\|\boldsymbol{x}-\boldsymbol{x}^{\prime}\right\|
$$

Likewise a pair of points $\left(p_{1}^{1}, p_{2}^{1}\right)$ is chosen from $P_{1}$, as a constraint, however, we require that their types correspond to the types $t_{1}^{0}$ and $t_{2}^{0}$ of points chosen from $P_{0}$. 
Then, an affine operator $\boldsymbol{A}=\left[a_{i j}\right], i, j \in\{1,2\}$ that maps the spatial coordinates $\left(\boldsymbol{x}_{1}^{1}, \boldsymbol{x}_{2}^{1}\right)$ to $\left(\boldsymbol{x}_{1}^{0}, \boldsymbol{x}_{2}^{0}\right)$ is estimated and applied to the spatial coordinates of all points in $P_{1}$ yielding a new point set $\boldsymbol{A}\left(P_{1}\right)$.

Subsequently, the sets $P_{0}$ and $\boldsymbol{A}\left(P_{1}\right)$ are aligned according to the distance $d$. This requires to know the number $n$ of type equivalent points in $P_{0}$ and $\boldsymbol{A}\left(P_{1}\right)$. It can be computed from a multiset intersection ${ }^{1} n=\left|P_{0, T} \cap P_{1, T}\right|$ of the multisets of interest point types where $P_{i, T}=\left\{t \mid(\boldsymbol{x}, t) \in P_{i}\right\}$.

Given $n, n$ pairs of points are estimated such that

$$
d\left(p_{1}^{0}, p_{1}^{1}\right) \leq d\left(p_{2}^{0}, p_{2}^{1}\right) \leq \ldots \leq d\left(p_{n}^{0}, p_{n}^{1}\right)
$$

where $p_{i}^{0} \in P_{0}$ and $p_{i}^{1} \in \boldsymbol{A}\left(P_{1}\right)$. Afterwards, the error

$$
E=\frac{1}{2 n} \sum_{i}\left\|\boldsymbol{x}_{i}^{0}-\boldsymbol{A} \boldsymbol{x}_{i}^{1}\right\|^{2}
$$

is calculated and $\boldsymbol{A}=\left[a_{i j}\right]$ is updated according to

$$
a_{i j}(\tau+1)=a_{i j}(\tau)-\frac{\partial E(\tau)}{\partial a_{i j}(\tau)} .
$$

These steps are iterated until $E$ falls below a certain threshold, does not improve anymore, or a maximum number of iterations is reached. Two sets of interest points are assumed to be equivalent if, after the iterations have terminated, $E$ is smaller than the threshold.

All this amounts in the algorithm shown in Fig. 5.3(a). The parameters were adjusted experimentally; $\tau_{\max }=20, \theta=0.05$, and $\epsilon=10^{-4}$ have proven to be suitable values.

We also tried two variants of this algorithm, the first one is depicted in Fig. 5.3(b). Here, the set $P_{1}$ is updated to $\boldsymbol{A}\left(P_{1}\right)$ at the end of each iteration. Consequently, the operator $\boldsymbol{A}$ has to be reestimated at the beginning of the next iteration. The second variant is not shown here for it is similar to the first one. The only difference is that the operator $\boldsymbol{A}$ will be updated a couple of times to $\boldsymbol{A}=\boldsymbol{A}+\Delta \boldsymbol{A}$ before $P_{1}$ is set to $\boldsymbol{A}\left(P_{1}\right)$. Both variants, however, resulted in heavy oscillations of the error $E$ and performed less well in practice. Details can be found in [33].

\section{Computing the Haussdorff Distance between Sets of Interest Points}

The Hausdorff distance between two point sets $P_{0}$ and $P_{1}$ depends on a method to measure the distance between individual points. Considering the interest point distance $d:\left(\mathbb{R}^{2} \times T\right)^{2} \rightarrow \mathbb{R}$ as defined above the Hausdorff distance between $P_{0}$ and $P_{1}$ is

$$
H\left(P_{0}, P_{1}\right)=\max \left(h\left(P_{0}, P_{1}\right), h\left(P_{1}, P_{0}\right)\right)
$$

\footnotetext{
${ }^{1} \mathrm{~A}$ multiset is a collection of objects that are not necessarily distinct [77]. For instance, $A=$ $\{a, b, b, c, c, c\}$ and $B=\{a, a, b, b, c, c\}$ are multisets. Their intersection is $A \cap B=\{a, b, b, c, c$,$\} .$
} 


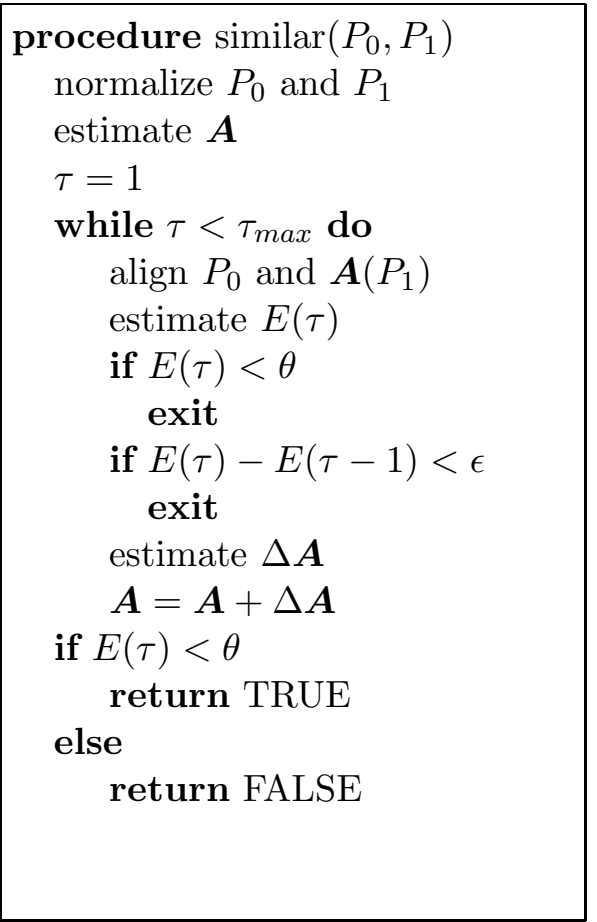

(a) Original method

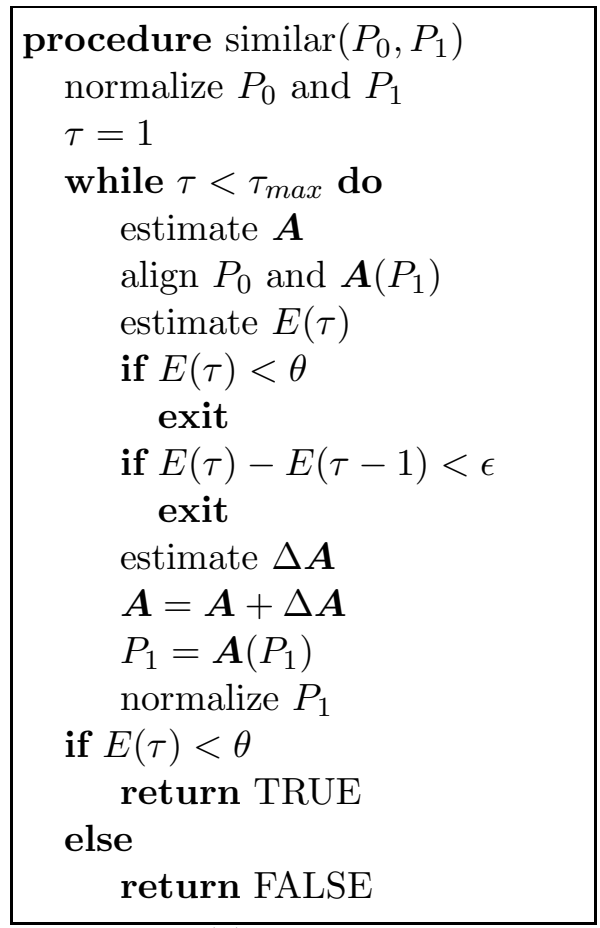

(b) A variant

Figure 5.3.: Algorithm to estimate the similarity of two sets of interest points by means of a gradient descent method.

where

$$
h\left(P, P^{\prime}\right)=\max _{p \in P} \min _{p^{\prime} \in P^{\prime}} d\left(p, p^{\prime}\right) .
$$

To test the equivalence of two sets of interest points the image coordinates $\boldsymbol{x}$ of all points in the sets are transformed to principle axis coordinates and the Hausdorff distance is computed subsequently. If it is smaller than a threshold $\theta=1.0$ that was estimated from test samples, the sets are said to represent the same object. Again, details are found in [33].

\section{A simple Voting Scheme}

It turned out that the gradient descent method and the Haussdorff distance tend to yield different results when applied to the same data. Thus, a simple majority voting scheme (cf. [96]) was realized to increase the reliability of point set matching.

Since majority voting needs at least three cues, we were in need of another index of point set similarity. As computing the distance between point sets by means of an affine 
operator mainly concerns image coordinates while computing the Hausdorff distance concerns image coordinates and mating feature types, we opted for another rather simple cue that puts emphasis on the type of interest points.

Given two interest point sets $P_{0}$ and $P_{1}$ of sizes $n_{0}=\left|P_{0}\right|$ and $n_{1}=\left|P_{1}\right|$, we again consider the number $n$ of type equivalent points contained in the sets. If, without loss of generality we assume that $n_{0} \leq n_{1}$ and if $n \geq 0.7 n_{0}$ then $P_{0}$ and $P_{1}$ are said to be equal.

Now there are enough cues for majority voting and two sets of interest points are believed to represent the same assembly if at least two of the three described methods vote accordingly, i.e. if at least two of them yield that $P_{0}$ and $P_{1}$ are equivalent.

\section{Results}

Figure 5.4 shows that, compared with syntactic analysis, interest point matching is beneficial in terms of computation time. The figure displays the average time needed to derive mating feature graphs or to test for point set equivalence. Both are sketched with respect to the number of bolts comprised in an assembly which indicates the complexity of the task. Obviously, the amount of time required for syntactic image analysis tends to grow exponentially. Generally, this is due to the fact that syntactic methods examine local properties of patterns. In our case, the semantic network for cluster parsing analyzes local adjacency relations among the objects in a cluster. The more adjacencies there are the more syntactic derivations must possibly be checked. If a chosen alternative fails to yield a good explanation (because too many objects in the cluster do not fit into the current structure), it has to be discarded and another description must be considered. Interest point matching, in contrast, deals with global properties of an assembly. It does not aim at explaining local relations between parts but regards distances between sets of features coordinates. If these sets are of reasonable size (in our experiments, an assembly with e.g. nine bolts typically had about 35 interest points), combinatorial explosions can be avoided.

To determine the recognition accuracy of voted point set matching an independent test set of 193 pairs of corresponding but geometrically different interest point sets was considered. For 153 of those pairs the similarity was recognized, i.e. this approach reaches a correctness of $79 \%$.

This seems a rather poor performance. However, our method for point set matching is embedded in a larger system. And user interaction with the system can defuse this problem. If neither region based matching nor interest point matching detects a correspondence to an assembly in the memory, a new syntactical description is computed and the regions, the interest points, and the syntactic structure are stored and interrelated. A user then can assign a name to this collection of data. If he chooses a name already contained in the memory, the system has learned another prototypical description of an already known assembly. The following discussion of an exemplary interaction with the memory shall illustrate this mechanism. 


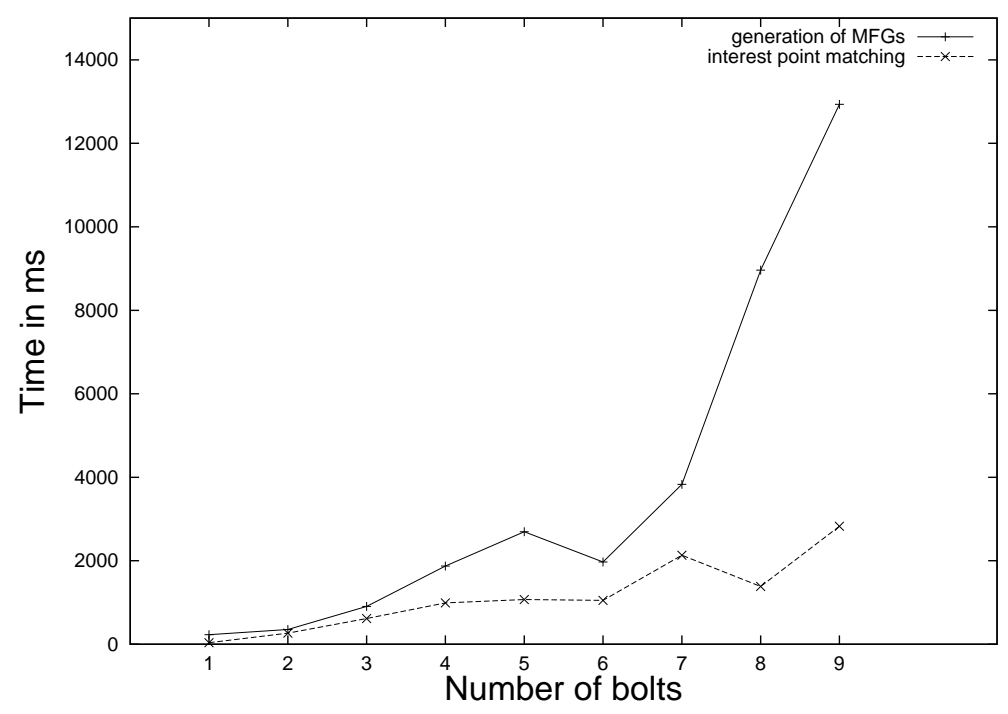

Figure 5.4.: Average computation times for generating a mating feature graph and matching two interest point clusters. Times are plotted versus the complexity of composite objects measured by the number of bolts comprised in an assembly. All experiments were carried out on a DEC AlphaStation 500/500 (SPECint95 15.0, SPECfp95 20.4).

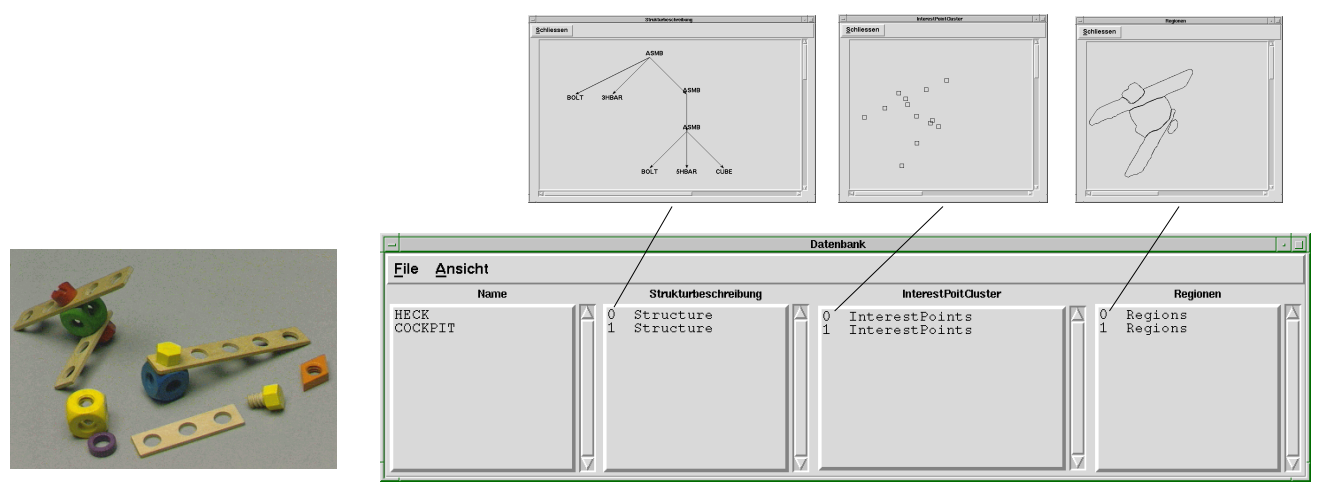

Figure 5.5.: Initial state of an assembly cell and content of the memory after the scene was analyzed.

\subsubsection{A Performance Example}

Figures 5.5-5.7 display a series of intermediate states of a construction process together with the corresponding content of the memory. Initially, there were two assemblies in the scene and the memory did not contain any data. Consequently, conventional assembly 


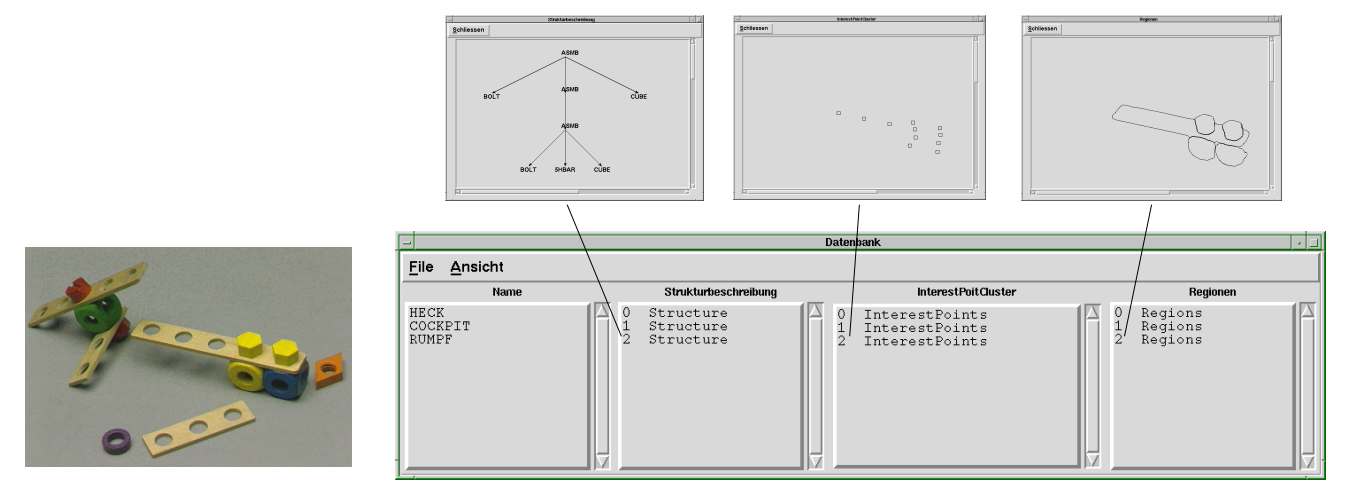

Figure 5.6.: Assembly cell viewed after a series of assembly tasks were carried out and corresponding state of the memory.
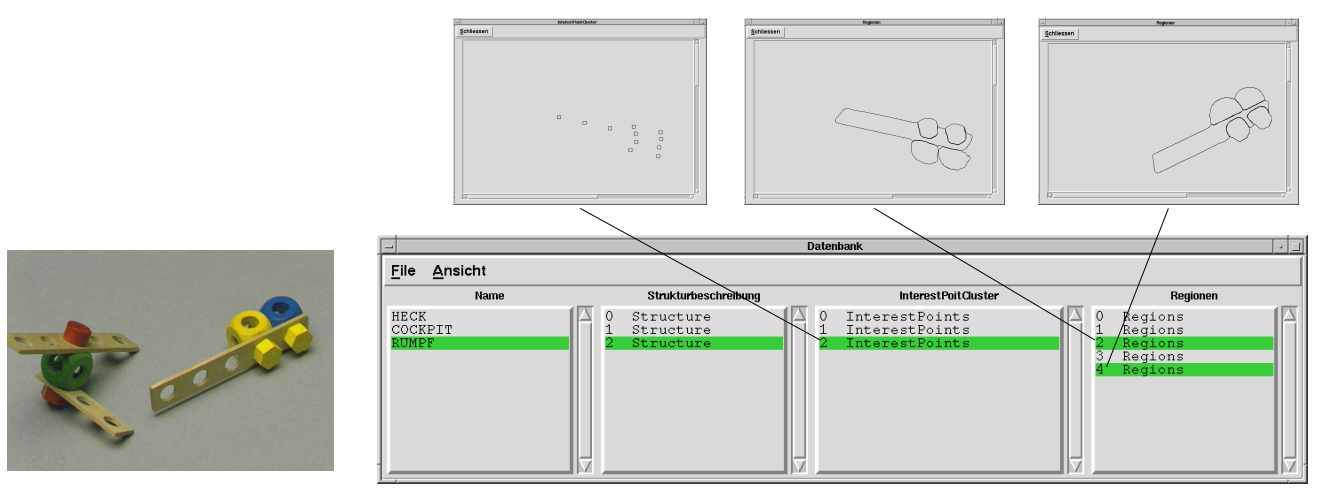

Figure 5.7.: The scene viewed, after the assemblies were rearranged and the corresponding memory content.

detection was carried out and the information gathered was accumulated in the memory. The region clusters depicting assemblies, sets of interest points found therein, and syntactic descriptions of both assemblies were stored in the corresponding dimension and interrelated correspondingly. Furthermore, a user assigned a name to each of the assemblies. One was called HECK, the other was referred to as COCKPIT. The scene and the resulting state of the memory are shown in Fig. 5.5. The cluster of regions, the set of interest points, and the syntactic structure that were stored as features of the HECK are visualized in the three windows on the top.

A series of assembly tasks resulted in the scene shown in Fig. 5.6. As the HECK did not change its appearance in the image, its new cluster of regions was successfully matched against one of the clusters already contained in the memory. Hence, the HECK was recognized and no further computations were necessary. For the assembly that newly appeared in the scene neither a corresponding cluster nor a similar point set were found 
in the memory, so, both kinds of information had to stored, a syntactic structure was determined, and it was called RUMPF. And of course, all this data, visualized in the top three windows in the figure, was linked correspondingly.

In the final scene, HECK and RUMPF were rearranged. Therefore, clusters of regions were extracted from the image to whom no corresponding ones were found in the respective memory dimension. Consequently, new clusters had to be registered. However, new sets of interest point did not have to be stored since the system found correspondences to already known ones. Like in the two preceding figures, the large window in the middle of Fig. 5.7 depicts the visual front-end of the memory which allows to examine the content of the different dimensions. Clicking on the entry RUMPF in the leftmost column of the window caused all the data related with it to be highlighted in green.

\subsection{Summary}

Earlier in this thesis we demonstrated that assemblies can be recognized from computer vision using a framework of syntactic and graph based methods. However, in a scenario of cooperative flexible assembly there are two rather antipodal aspects that should be considered. On the one hand, syntactic image parsing and subsequent graph matching are very flexible but might be time consuming if the assemblies in the scene are of high complexity. On the other hand, mechanical assembly usually is a step by step procedure of building complex objects from simpler ones. And in most of the tasks that can be observed during a process only a few objects are manipulated.

Thus, in order to avoid unnecessary efforts, assembly process monitoring by means of computer vision should incorporate knowledge of the history of an assembly process. It should consider recognition result accomplished for objects that were manufactured earlier.

This chapter presented an idea how to extend our structural framework by methods that approach assembly recognition from a different direction. We introduced an architecture for a visual memory intended to store and interrelate information from different levels of abstraction gathered during syntactic image analysis. The basic idea was that visual assembly features which were stored at an earlier stage of a construction process might serve to quickly recognize assemblies in subsequent stages and thus might help to supersede time consuming parsing.

The characteristics of the proposed architecture are multidimensionality, scalability, modularity, and hybridicity. The memory is multidimensional since it registers data from different stages of image processing. If new information is drawn from an image, it is stored and related to earlier derived facts. By this the memory dynamically learns prototypical features for assembly recognition. It is scalable since new dimensions can easily be integrated into the architecture. Finally, it is a hybrid and modular system 
because it integrates different methods for recognition and the techniques to compare data of a certain dimension can be easily exchanged.

The implemented experimental version of the memory has four dimensions. These are used to store clusters of labeled image regions, sets of interest points, syntactic descriptions, and names. While the former two types of data represent image features, the latter two are of symbolic nature. With a content like this, there is an implicit hierarchy of the dimensions of the memory. Information in the higher dimensions is derived from or assigned to data in the lower ones. This led to an extension of the algorithm for assembly detection.

Before further analyzing an input cluster of objects, a corresponding one will be searched in the lowest dimension of the memory. If there is a similar one, assembly recognition has succeeded. If there is none, the cluster is stored in the memory and interest points are calculated. If a corresponding set of interest points can be found, it will be related to the recently registered cluster and recognition has succeeded. Otherwise, the new set will be integrated in the memory and syntactic cluster parsing will begin.

While the implemented method for object cluster comparison is coarse, the realized approach of interest point set matching is more sophisticated. It was evaluated with respect to computation time and accuracy. It proved that point matching speeds up visual assembly recognition and does not depend exponentially on the complexity of the input. However, its accuracy is rather moderate. But we argued that in a scenario of intelligent man-machine interaction for assembly this is not a severe drawback. If the system fails to recognize an assembly properly, a user can correct its conclusions and the system can use this correction to further extend its knowledge.

Interesting directions for future work are first of all the integration of a memory dimension that stores mating feature graphs. Furthermore, we plan the investigation and integration of other visual assembly features. Contour information, for instance, seems to be an auspicious candidate since, within the SFB 360, there already is experience with its use for object recognition [116]. And of course, other methods for object cluster and interest point set comparison should be taken into consideration. 


\section{Evaluation}

In Chapter 3, we exhaustively discussed the use of context free grammars in assembly modeling. We explained that context free assembly grammars can be implemented as semantic networks using ERNEST and introduced a control algorithm that parses for assembly structures in nonlinear input. This algorithm was inspired from principles of discourse parsing and examples of its application in the detection of baufix ${ }^{\circledR}$ assemblies from image data and in structuring sets of three-dimensional furniture models were presented.

For the baufix ${ }^{\circledR}$ scenario we also discussed the integration of cues from syntactic context into elementary object recognition. And we presented a method to generate high-level assembly sequence plans from image parsing. These plans were more detailedly studied in Chapter 4 where we used them to compute graph based representations of assemblies which in turn are employed in recognition or reconstruction.

Hence, grammar based assembly detection by means of a semantic network substantially contributes to the perceptive capabilities of the artificial communicator the SFB 360 intends to develop. In spite of the little effort for modeling, it is flexible for it finds arbitrary assembly structures. Furthermore, it provides valuable cues for further steps of processing. Integrated into a system for multi-modal interaction in assembly, assembly detection is not a stand alone component but supplies significant information to other processing modules. However, up to now we did not quantify how accurate syntactic assembly detection is in practice. This shall be done next.

In the following, we will first present a couple of prototypical results obtained from image parsing by means of the ERNEST network introduced in Chapter 3.2.2. They shall point out that out parser successfully copes with the problem of over-generalization which we discussed when introducing the idea of grammatical assembly modeling in Chapter 3. Also based on these examples, we will argue that the performance in detecting baufix ${ }^{\circledR}$ assemblies is task dependent. Consequently, we will plead for an evaluation of our algorithm that takes into account how difficult the data is it has to deal with.

However, assessing the difficulty of a pattern recognition task is difficult itself. The notion of difficulty often is rather subjective and fuzzy and in our context it is not at all clear how to measure it. Due to its inherent fuzziness we decided to identify a couple of indices of task difficulty and to apply a fuzzy function to classify it. We will discuss this idea in more detail and then present the results we could achieve within this framework. 


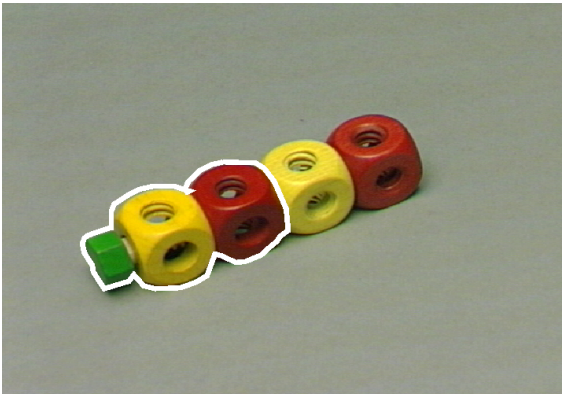

(a) Difficulty 0.32

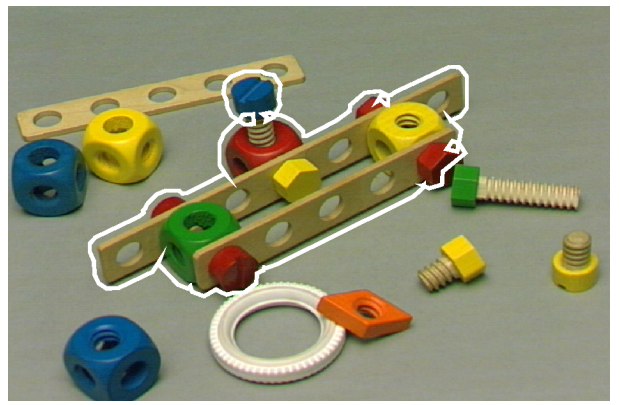

(c) Difficulty 0.72

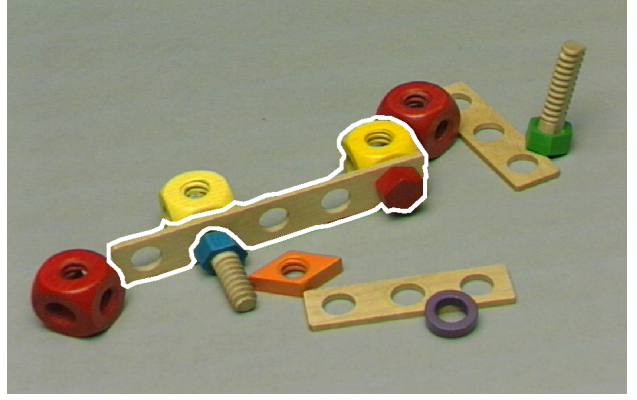

(b) Difficulty 0.53

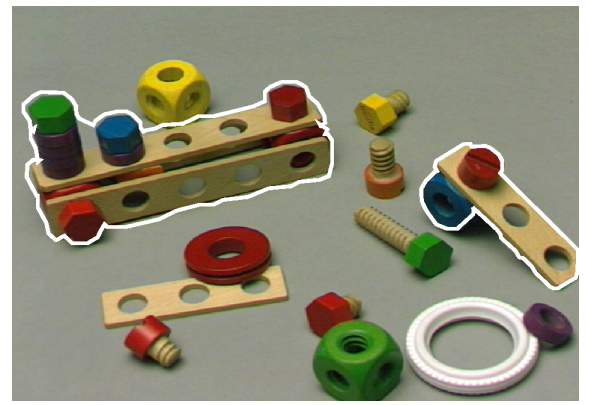

(d) Difficulties 0.72 and 0.16

Figure 6.1.: Detection results obtained from object clusters of varying difficulty.

\subsection{Prototypical Detection Results}

Figure 6.1 displays prototypical detection results for the baufix ${ }^{\circledR}$ domain. Each image depicts one or several clusters of objects as they usually appear in a flexible and unconstrained assembly environment. The largest assembly structures found in a cluster of objects are enclosed by white polygons.

Some of the examples testify that the semantic network based parsing algorithm successfully copes with problems we first mentioned on page 31 in Chapter 3. There, we discussed that the grammar that models the class of baufix ${ }^{\circledR}$ assemblies also derives structures which are mechanically infeasible. In particular, we explained that due to its recursive properties the grammar derives structures of infinitely many objects attached to a single bolt. But from the example in Fig. 6.1(a) we see that in practice we do not have to worry about effects like this.

In Fig. 6.1(a), only two out of the chain of four cubes were associated with the bolt. But this is perfectly correct for the bolt's thread is not long enough to carry more than two cubes. Information like this can be modeled by means of attributes of the semantic network. In this example, syntactic cluster parsing correctly terminated after 
instantiating the assembly consisting of the green bolt, a yellow and a red cube because an attribute that registers the state of mating features yielded that the bolt could not pick up further parts.

Another type of contextual constraint our implementation can deal with is exemplified in Fig. 6.1(b). Although the yellow cube and the blue bolt enclose the detected assembly as if it were a complex miscellaneous part, the parser yielded that they are not connected. The obvious reason is that the thread of the bolt points away from the assembly and the cube. Geometric information like this cannot be captured in a context free grammar but again attributes defined in the semantic network will help.

The examples also indicate that, in our scenario, assembly detection has to deal with images of varying complexity. Hence, it is no surprise to observe that the performance in assembly detection depends on the complexity of the input or on the difficulty of the task. Certainly, this figure will be influenced by several factors. For instance, visual assembly detection surely depends on the visibility of the objects that form an assembly. The more parts are occluded the more likely it is to gain a fairly wrong description. But even if objects are just partially occluded, it may have consequences. Objects that are hardly visible might not be recognizable or they might not be recognized as parts of a partial order and thus be ignored by the parser.

This dependency on the input complicates the provision of meaningful test sets to assess our algorithm. If a test set contained too many simple cases, the parsing algorithm might be overrated. If the test set contained too many difficult images, the observable performance might be below average. We therefore decided to evaluate our approach with respect to the difficulty of the input.

\subsection{Assessing the Difficulty of a Task}

The basic idea to assess the difficulty of a task was to consider influencing factors that can be measured from an image. Provided a suitable method this would allow to compute the difficulty instead of annotating it manually. Assuming a cluster of labeled image regions $C$ to be given, we opted for the following four features:

- the number $\mathcal{B}(C)$ of visible bolts in $C$

- the number $\mathcal{P}(C)$ of visible parts in $C$

- the mean number $\mathcal{A}(C)$ of adjacent objects in $C$

- the mean degree $\mathcal{O}(C)$ of perspective occlusion in $C$

Note that although intuitively they would be interesting, the total numbers of parts and bolts of an assembly do not appear in this list for they cannot be guaranteed to be measurable: parts that are not visible cannot be counted from an image. 
However, each of the listed indices was chosen according to considerations mentioned earlier. As explained in Chapter 3 only clusters of objects can depict assemblies thus only clusters of objects have to be searched for assembly structures. Therefore, it must be properties of object clusters that influence assembly detection. From Fig. 5.4 on page 113 we know that the number of visible bolts in a cluster has an impact on the time needed for parsing. The number of parts comprised in an assembly is generally accepted as an index of complexity $[55,136]$; measurable from an image is the number of visible parts. Likewise, it is agreed that assembly topology determines how many syntactic structures there may be. For the baufix ${ }^{\circledR}$ domain, we verified this at the beginning of Chapter 4 ; the mean number of adjacent objects in a cluster is meant as a measure of connectedness. Finally, we just discussed that part visibility is of crucial influence for assembly detection.

While it is trivial to obtain the first two features $\mathcal{B}(C)$ and $\mathcal{P}(C)$ from a given cluster of objects $C$, the computation of $\mathcal{A}(C)$ and $\mathcal{O}(C)$ is not that obvious but nevertheless simple. The mean number of adjacent objects in a cluster is defined as

$$
\mathcal{A}(C)=\frac{1}{\mathcal{P}(C)} \sum_{p \in C} \mathcal{A}(p)
$$

where $\mathcal{A}(p)$ is the number of objects adjacent to a part $p \in C$. And the mean degree of perspective occlusion is given by

$$
\mathcal{O}(C)=1-\frac{1}{\mathcal{P}(C)} \sum_{p \in C} v(p)
$$

where

$$
v(p)=\left\{\begin{array}{cl}
\frac{s(p)}{\bar{s}(p)} & \text { if } \frac{s(p)}{\bar{s}(p)}<1 \\
1 & \text { otherwise }
\end{array}\right.
$$

denotes the degree of visibility of a part $p$ and $s(p)$ denotes the size of $p$ measured in pixels. The denominator $\bar{s}(p)$ represents the average size the part $p$ would have if it was not occluded by any other part; suitable values for $\bar{s}$ were determined empirically.

Thus, each cluster of labeled image regions $C$ passed to the assembly detection algorithm can be characterized by a feature vector

$$
\boldsymbol{x}(C)=(\mathcal{P}(C), \mathcal{B}(C), \mathcal{A}(C), \mathcal{O}(C)) .
$$

For the object cluster in Fig. 6.1(a), for instance, a vector $\boldsymbol{x}_{a}=(5,1,1.6,0.19)$ was computed while the assembly in Fig. 6.1(b) is part of a cluster that yielded $\boldsymbol{x}_{b}=$ $(11,3,2,0.17)$. However, even though except for the degree of occlusion the feature values in $\boldsymbol{x}_{b}$ are higher than the ones in $\boldsymbol{x}_{a}$, such vectors do not tell much about difficulty. Yet most people would agree that for the scene in Fig. 6.1(b) it seems more demanding to find an assembly than for the one in Fig. 6.1(a). And indeed, on a DEC AlphaStation 

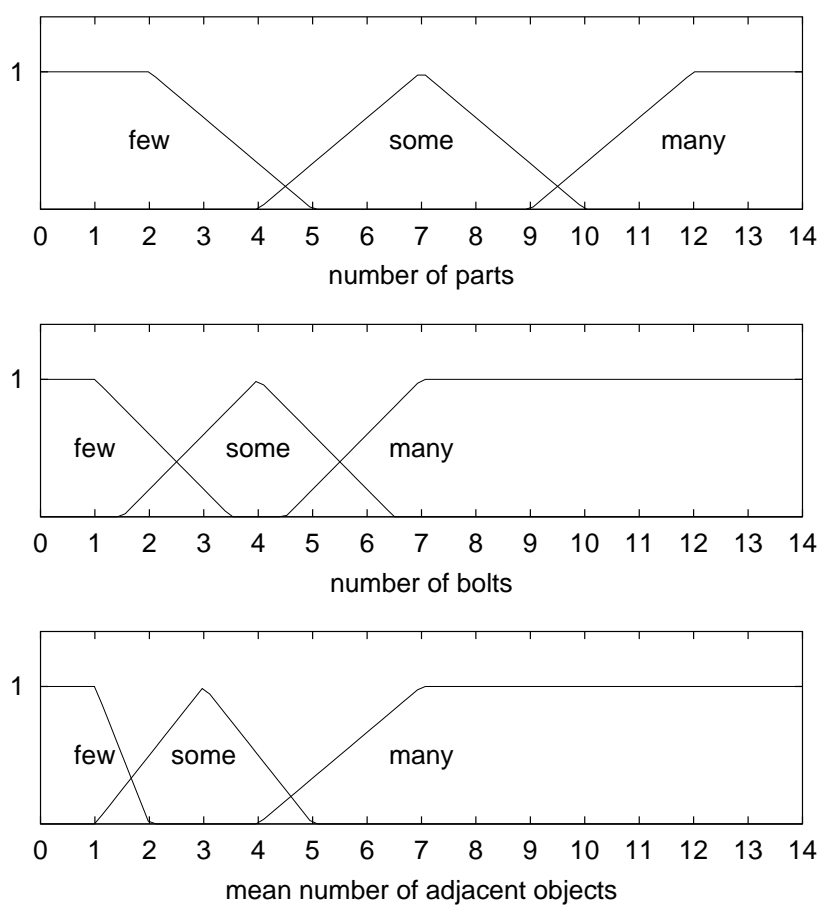

Figure 6.2.: Fuzzy sets and corresponding linguistic values to partition the ranges of the measures $\mathcal{P}(C), \mathcal{B}(C)$, and $\mathcal{A}(C)$.

$500 / 500^{1}$ assembly detection took $2944 \mathrm{~ms}$ for the cluster in Fig. 6.1(b) while for the one in Fig. 6.1(a) the result was available after $339 \mathrm{~ms}$. But this still does not tell how difficult assembly detection was. We therefore are in need of a technique that derives a measure of difficulty from a common sense assessment of cluster complexity. Fuzzy logic is a well accepted method to do so.

In order to quantify the task difficulty we thus applied a simple fuzzy classification scheme originally introduced by Mamdani and Assilian [80]. In this approach, the range of possible values of a variable $X$ is partitioned by a set of fuzzy sets. Each fuzzy set $F$ is then assigned a linguistic value like for instance few, some, or many so that a numeric value $x$ can be characterized by its degree of membership $\mu_{X, F}(x)$ to a linguistic value.

Figures 6.2 and 6.3 show the fuzzy sets and the corresponding linguistic values we did choose to partition the influencing factors and the difficulty itself.

Furthermore, rules have to be provided that characterize the relation between a collection of measurements and the entity these measurements represent. This is done

${ }^{1}$ SPECint95 15.0, SPECfp95 20.4 

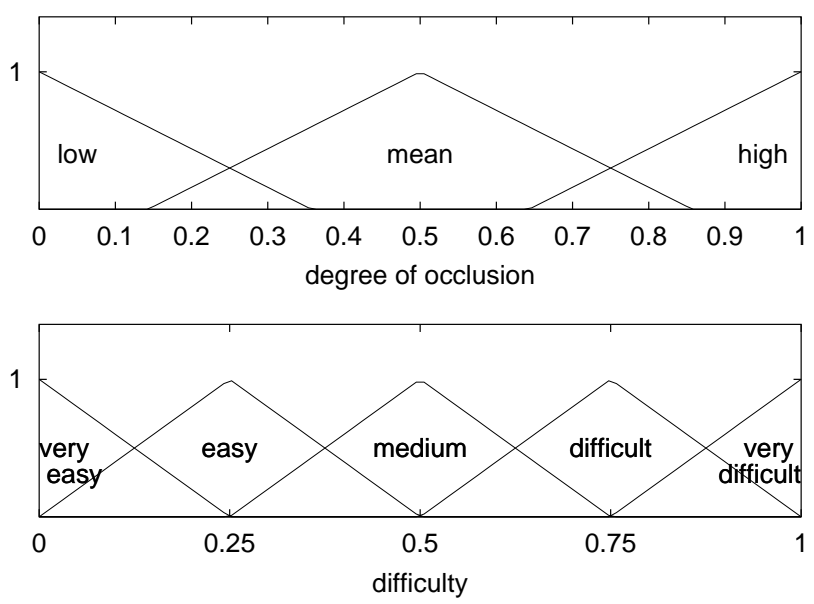

Figure 6.3.: Fuzzy sets and corresponding linguistic values to partition the ranges of the measure $\mathcal{O}(C)$ and the difficulty $D$.

\begin{tabular}{cccc|c}
$\mathcal{P}(C)$ & $\mathcal{B}(C)$ & $\mathcal{A}(C)$ & $\mathcal{O}(C)$ & difficulty \\
\hline few & few & few & low & very easy \\
few & few & few & mean & easy \\
few & few & some & low & easy \\
few & few & some & mean & easy \\
few & some & few & low & easy \\
$\vdots$ & $\vdots$ & $\vdots$ & $\vdots$ & $\vdots$ \\
many & many & many & high & very difficult
\end{tabular}

Table 6.1.: Table of fuzzy rules to classify the difficulty of assembly detection.

by means of tables containing linguistic values. Table 6.1 lists some of the rules we used to model our intuition about the difficulty of assembly detection. From the first row, for instance, we see that if a cluster contains a few objects and a few bolts and there are few adjacent objects at an average and the degree of perspective occlusion is low, then the task will be very easy.

The quantitative classification of a given feature vector $\boldsymbol{x}$ happens as sketched in Fig. 6.4. First, for each rule in the rule base the degree of membership of the numeric components $x_{i}$ of $\boldsymbol{x}$ to the values in the rule's antecedent is estimated. For example, 


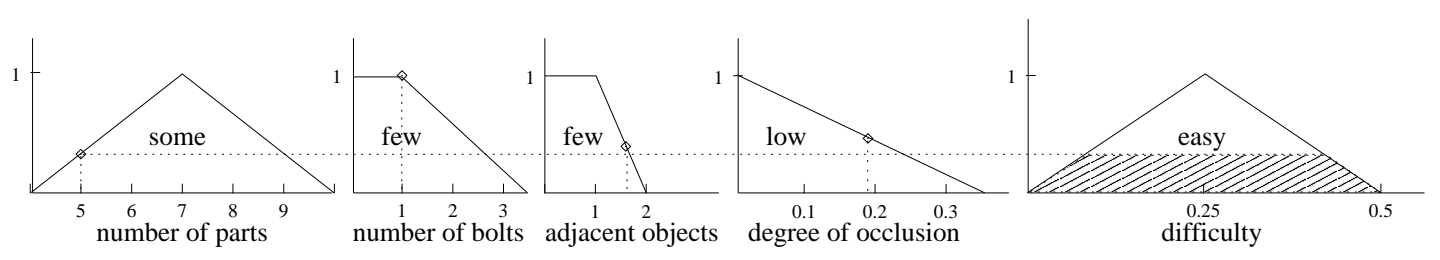

(a) For $\boldsymbol{x}_{a}=(5,1,1.6,0.19)$ rule $r_{9}$ yields $\mu_{r_{9}}=(0.33,1,0.4,0.468)$ with $\alpha_{r_{9}}=0.33$

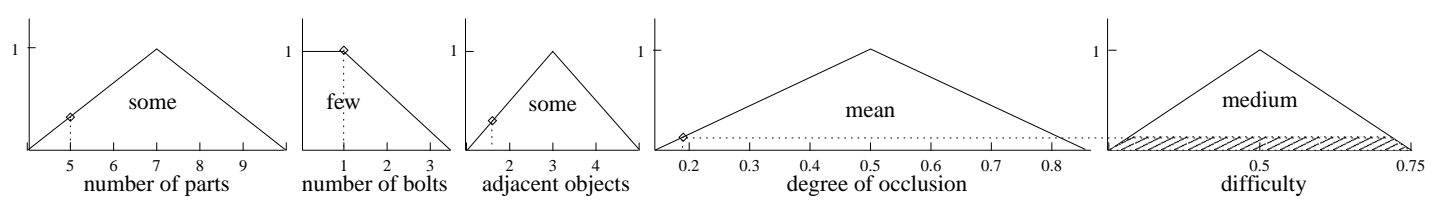

(b) For $\boldsymbol{x}_{a}=(5,1,1.6,0.19)$ rule $r_{12}$ yields $\mu_{r_{12}}=(0.33,1,0.3,0.132)$ with $\alpha_{r_{12}}=0.132$

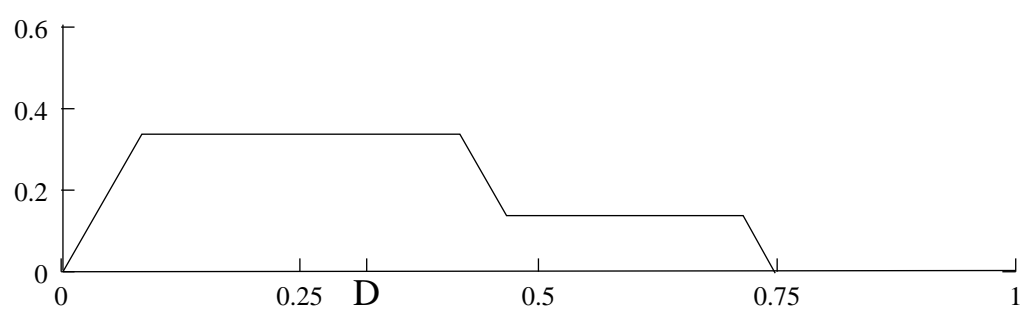

(c) Merged results from 6.4(a) and 6.4(b)

Figure 6.4.: Fuzzy classification according to Mamdani and Assilian [80]. For the feature vector $\boldsymbol{x}_{a}$ computed from Fig. 6.1(a) there are two firing rules. These produce the output set in Fig. 6.4(c). The difficulty $D=0.32$ of the task characterized by $\boldsymbol{x}_{a}$ corresponds to the horizontal component of the center of the output set.

given the vector $\boldsymbol{x}_{a}=(5,1,1.6,0.19)$ derived from Fig. 6.1(a), for the first rule $r_{1}$ of Tab. 6.1 we would have to compute

$$
\mu_{r_{1}}\left(\boldsymbol{x}_{a}\right)=\left(\mu_{\mathcal{P}, \text { few }}(5), \mu_{\mathcal{B}, \text { few }}(1), \mu_{\mathcal{A}, \text { few }}(1.6), \mu_{\mathcal{O}, \text { low }}(0.19)\right) .
$$

If for a rule $r$ we have $\mu_{X_{i}^{r}, F_{i}^{r}}\left(x_{i}\right)>0$ for each component $x_{i}$ of $\boldsymbol{x}$, the rule is said to be firing. Its degree of acceptance $\alpha_{r}$ is defined as

$$
\alpha_{r}=\min _{i} \mu_{X_{i}^{r}, F_{i}^{r}}\left(x_{i}\right)
$$

and subsequently used to clip the fuzzy set in the consequence of $r$ (see Fig. 6.4). Merging the clipped consequences of all firing rules yields the the output set of the method by Mamdani and Assilian.

Given the fuzzy output set, there are several methods to obtain the numeric classification result one is interested in. In our experiments, we applied the so called center of 


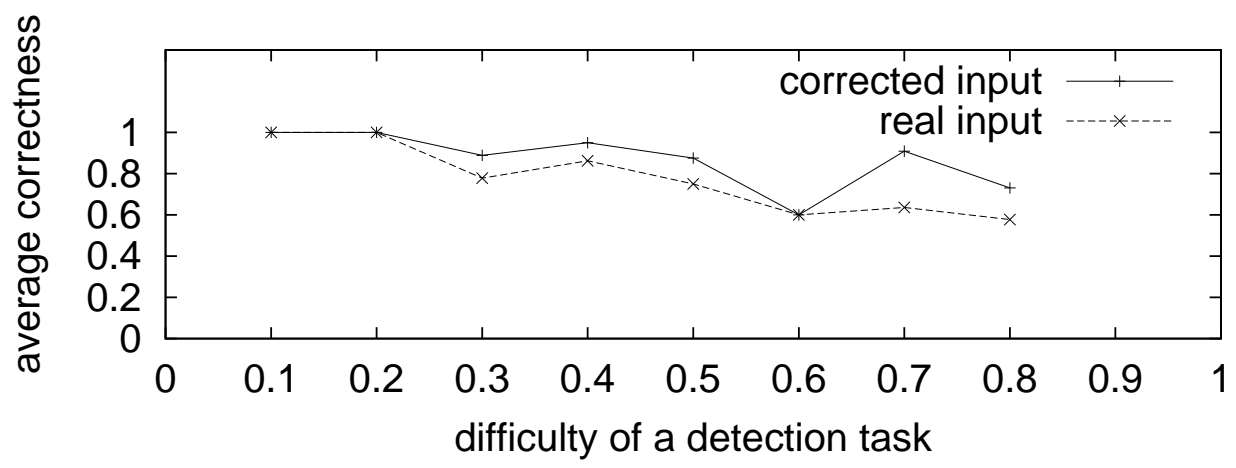

Figure 6.5.: Detection accuracy with respect to the difficulty of the task.

area method which yields the numeric value as the horizontal component of the center of gravity of the fuzzy output. As we see from Fig. 6.4(c), for the feature vector $\boldsymbol{x}_{a}$ we considered as an example this resulted in a task difficulty $D=0.32$.

\subsection{Results}

The partitions of the influencing variables as well as the fuzzy rules that characterize interdependencies among them which are shown in Fig. 6.2 and 6.3 and Tab. 6.1 were adjusted according to a training set of 70 object clusters.

Figure 6.5 summarizes the results we obtained with this parameterization. To produce this figure the difficulties of 164 clusters of labeled image regions from an independent test set were estimated and rounded to values of $0.1,0.2, \ldots, 0.8$. Examples of difficulties that could have been rounded to other values were not contained in the test sample.

Two series of experiments were carried out. In the first series, we manually corrected region labels resulting from elementary object recognition so that the input to the assembly parser was always correct. This was done to estimate the optimal performance in assembly detection. If there were errors and misclassifications in the input, cluster parsing simply cannot produce correct results. A second series was done without manual corrections and thus represents the real world testbed. In both series, an experiment was counted a success if syntactic analysis resulted in the mating feature graph one would expect from what was visible in the input.

Evidently, simple data was always processed correctly whereas correctness diminishes with increasing difficulty. But even in difficult cases syntactic assembly detection is quite accurate and the difference between results obtained from manually corrected data and real input is small. The latter actually shows that object recognition according to Kummert et al. [73] provides reliable input to our assembly detection method. Viewed from the perspective of a system for cooperative construction in an unconstrained en- 


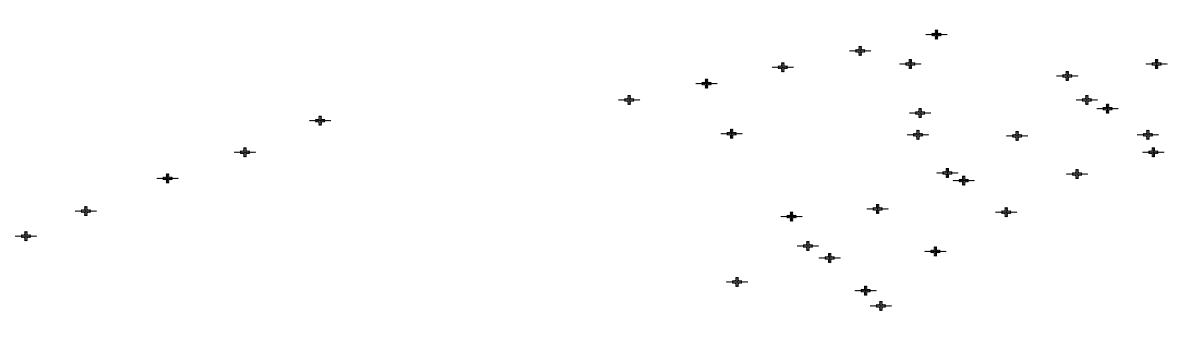

(a)

(b)

Figure 6.6.: Distributions of calculated mating feature coordinates for the object cluster in Fig. 6.1(a) and the large cluster in Fig. 6.1(c).

vironment, these results are encouraging. For scenes of moderate complexity, syntactic assembly detection yields very reliable results. It thus fits well into an architecture for multi-modal man-machine interaction whose other components process input of similar complexity [7].

An analysis of the unsuccessful detection attempts revealed that failures were due to the assumptions concerning mating features we made on page 52 in Chapter 3.3.1. There, we presented a method to roughly approximate image coordinates of mating features. We explained that lines of those coordinates reflect partial orders among objects attached to bolts and that object cluster parsing is performed along these lines. Now, we discovered two basic reasons for detection errors closely related to this heuristic.

The first one may affect any cluster independent of its difficulty: especially for the features of bars the calculated coordinates often deviate from their real position. For certain views, this may lead to lines of coordinates which do not correspond to any object order actually depicted in an image. For instance, instead of being situated between a bolt and a nut, the hole of a bar might wrongly be assumed to be in front of the bolt. Pathological cases like this obviously prevent correct detection.

The second type of error explains why detection accuracy decreases with a growing difficulty. It mainly affects clusters of many objects which show a high degree of adjacency and thus come along with large and homogeneous sets of mating feature coordinates. Figure 6.6, for instance, contrasts the mating features of the object cluster in Fig. 6.1(a) with the feature distribution corresponding to the large cluster in Fig. 6.1(c). This example indicates that for clusters of higher difficulty there usually are many possibilities to group features into lines. Therefore, it is likely that a line is chosen for parsing that does not correspond to a partial order of interconnected objects. But if it neverthe- 
less constitutes an object sequence that complies with the assembly grammar, erroneous structures will result.

However, neither of these sources of error allows to raise fundamental criticism of our syntactic approach to visual assembly detection. On the one hand, context free grammar based assembly detection accomplishes very accurate results for input of minor and medium difficulty and still performs satisfyingly for difficult cases. On the other hand, the shortcomings we discussed are neither due to grammatical modeling nor to our semantic network based adaption of discourse parsing. Rather, they are caused by the assumptions and parameters which guide parsing. This actually provides an agenda for further work.

Obviously, detection failures of the first type could be avoided if there was a more precise method to estimate feature coordinates. Higher precision will hardly be obtainable from the kind of data we are dealing with up to now. But within our research project there is expertise in low-level vision techniques to detect subparts of objects. These techniques yield so called focus points which are used to support object recognition [112]. Similar methods might supply accurate feature coordinates to the assembly detection module. Errors of the second type result if the attributes of the semantic network that govern the instantiation process determine a wrong direction for parsing. These attributes, however, were parameterized according to a sample of 20 rather simple clusters (which are part of the samples for adjusting the fuzzy classifier but independent from the set for testing). Thus, readjusting these parameters with regard to more complex clusters might yield an improvement for difficult input.

Seen from this perspective, syntactic assembly detection based on simple grammars is rather robust and shows amazing generalization capabilities. In conclusion we can state that using a context free grammar for assembly modeling, implementing it as a semantic network, and adopting strategies from discourse parsing to realize a control algorithm meets the requirements posed by the constructions scenario we are dealing with. Our approach is generic and flexible, it treats different difficulties, and provides reliable data to other modules of an integrated system for man-machine interaction.

\subsection{Summary}

This chapter assessed the performance of cluster parsing. First, we presented some scenes of arbitrarily arranged parts from our cooperative construction scenario where visual assembly detection succeeded. They were meant to underline the advantages the semantic network language ERNEST offers as a tool for implementing parsing algorithms.

When we introduced our context free assembly grammar in Chapter 3, we pointed out that it actually is too general and derives structures which do not correspond to feasible assemblies. Later in that chapter, we presented the idea to implement context free grammars as semantic networks using ERNEST . We asserted that attributes defined 
in an ERNEST knowledge base will allow to cope with over-generalization for they can model features and properties of a concept that depend on its context. With regard to assembly detection from vision we claimed that they can model mechanical constraints not expressible in a context free grammar and thus may prevent infeasible structures to result from parsing. The results presented in this chapter indicated that this claim is justified.

Moreover, the examples indicated that syntactic assembly detection must handle input of different complexity. As this will make it difficult to provide meaningful test sets for an evaluation, we promoted the idea to estimate its performance with respect to the difficulty of the task. To this end we selected a set of four measurable features of a cluster of labeled image regions which exert influence on cluster parsing. We saw that this choice was plausible for it accords with observations presented in earlier chapters.

However, the difficulty of a task is a rather subjective notion. As a means to quantify it from a given collection of cluster features we discussed and applied a fuzzy classification scheme proposed by Mamdani and Assilian [80]. We presented suitable fuzzy set partitions of the influencing factors and a rule base that characterizes their impact on the difficulty, both drawn from a set of 70 exemplary object clusters. Given this parameterization, an evaluation of a test set of 164 object clusters revealed that the detection accuracy accomplished with our method indeed depends on the difficulty of the input. For input of minor difficulty the accuracy in assembly detection reaches $100 \%$ but decreases down to $60 \%$ in difficult or very difficult cases.

We discussed two major sources of error but saw that neither of these does challenge or question our approach in general. On the one hand, syntactic assembly detection performs satisfyingly even for difficult input and does not totally collapse. On the other hand, we could ascribe detection failures to the choice of a few internal parameters of our assembly parser. As they were chosen with respect to less difficult input, the performance for difficult input is in fact very well acceptable and there is reasonable hope that a careful adjustment of these parameters will gain better results for difficult cases.

Using a context free grammar for assembly modeling, a semantic network for implementation, and discourse parsing strategies for detection thus solves the problem of flexible assembly detection in an unpredictable environment. This framework provides a flexible, reasonable, and reliable means for the detection of assembly structures in image data. 


\section{Conclusion}

Recently, scientists investigating advanced human-computer communication discovered interactive toys and game playing scenarios as a suitable setting to develop and test their algorithms. The Collaborative Research Center 360 (SFB 360) at Bielefeld University is among those projects that pioneered this idea. It aims to develop a robot that instructed by spontaneous speech and gesture- assembles toy models from parts of the baufix ${ }^{\circledR}$ construction-kit. As the parts can be arbitrarily arranged in the construction cell and as its instructor should be free to decide what to assemble, the machine must be equipped with flexible algorithms to appropriately perceive and react to its surroundings.

Emerging from the SFB 360, this thesis addressed the problem of assembly recognition from computer vision. According to the underlying scenario a robust, reliable, and, of course, flexible solution was of primary interest. Due to the enormous variety of possible assemblies we argued that recognition must be based on generic knowledge representation. From a thorough review of assembly modeling and recognition literature we concluded that, as our scenario does not provide high precision sensors, syntactic methods best meet this requirement.

Simple concepts from formal language theory are sufficient to model the component structure of mechanical assemblies. To motivate this, we brought together ideas due to Homem de Mello and Sanderson and a discovery by Hall. While Homem de Mello and Sanderson established AND/OR graphs as a standard to represent all component structures of mechanical artifacts, Hall pointed out that AND/OR graphs and context free grammars as introduced by Chomsky are equivalent formalisms. Moreover, we demonstrated that understanding assemblies to be made from functional units results in compact grammars with recursive productions which derive entire classes of assemblies.

However, context free grammatical models of classes of assemblies cannot represent context dependent mechanical restrictions and thus may derive structures which are mechanically infeasible. But we proved that context free grammars are structurally equivalent to certain ERNEST knowledge bases. Therefore, they can be implemented as semantic networks using ERNEST and grammatical parsing can be realized as the instantiation of concepts of an ERNEST network. Furthermore, domain or context dependent knowledge can be modeled by means of attributes and will prevent the instantiation of infeasible assembly structures.

Intense cooperation with researchers working on spontaneous speech processing revealed a similarity between discourse and assembly structures. And ideas developed for 
discourse parsing inspired a robust instantiation strategy for the ERNEST based assembly detection in higher-dimensional input. Two exemplary implementations demonstrate the suitability of this approach. One searches clusters of labeled image regions resulting from elementary object recognition for structures corresponding to baufix ${ }^{\circledR}$ assemblies. The other detects certain furniture arrangements in sets of CAD models. As both implementations analyze and register mating relations among parts in the respective data, assembly detection using ERNEST actually yields high-level assembly sequence plans.

Besides syntactic assembly structures and plans grammar based parsing embedded in ERNEST can yield valuable cues for other recognition tasks. Integrated into a feedback loop along with a module for elementary object recognition, the assembly parser applies contextual knowledge to hypothesize labels for unrecognized image areas, to generate expectations that would complete partial structures, and to rate competing recognition results. Furthermore, there is successful mutual support with a module for action detection. While assembly detection supplies connection details to the action detection component, action detection yields accurate assembly sequences and thus allows to cope with less severe perspective occlusion which might affect assembly detection.

Syntactic assembly modeling suffers from combinatorial explosion. The number of different syntactic structures of a baufix ${ }^{\circledR}$ assembly grows exponentially with the number of bolts it contains. Thus, though grammatical parsing is well suited for flexible assembly detection, it is less suited for assembly recognition. Since parsing cannot be guaranteed to always yield the same syntactic structure of a complex assembly, syntax based recognition would require to store numerous individual descriptions or grammars.

However, high-level sequence plans resulting from syntactic assembly detection characterize topological relations among mating features of interconnected parts. These can be represented as a mating feature graph. As all different plans of an assembly will lead to the same graph, mating feature graphs are topologically unique assembly models. This observation revealed another striking correspondence between mechanical assemblies and ideas from formal language theory: we demonstrated that mating feature graphs constitute a denotational semantics of assembly sequence plans.

Based on mating feature graphs, assembly recognition is a problem of graph matching which we attacked using a software developed by Messmer and Bunke. Searching for subgraph isomorphisms between mating feature graphs enables assembly and subassembly recognition; searching for error-correcting subgraph isomorphisms copes with perspective occlusion and recognizes similar assemblies. Matching mating feature graphs derived from stereo images even enables $3 \mathrm{D}$ object reconstruction and thus allows to distinguish topologically identical assemblies of different geometry.

Assembly recognition by means of syntactic image parsing and subsequent graph matching is flexible but requires rather abstract data and might be time consuming. Therefore, we also examined assembly recognition based on information from lower levels of abstraction. The basic idea was to introduce a visual memory that stores and relates data from different stages of image processing. By matching results from a recent 
input image against earlier stored data, the memory either recognizes or learns visual characteristic of individual assemblies. The features currently considered are clusters of image regions, sets of interest points, syntactic structures and names assigned by a user.

Assembly recognition from a set of interest points was investigated more closely. We considered point set matching that couples the computation of an affine mapping using a domain specific gradient descent method with a variant of the Haussdorff distance. Experiments indicated that in contrast to parsing with subsequent graph matching the average computation time of this approach does not depend exponentially on the input; its recognition accuracy reaches $79 \%$.

As the scenario studied in the SFB 360 does not constrain the construction environment, visual assembly detection must cope with input of varying complexity. In order to avoid insignificant results we thus promoted the idea of a task dependent evaluation of assembly detection and mating feature graph generation. Given a set of features that exert influence on a detection task, the rather vague measure of task difficulty is quantified according to a fuzzy classification scheme introduced by Mamdani and Assilian. For input of minor difficulty the detection of assembly structures performs with an accuracy of $100 \%$ while for very difficult input the accuracy decreases down to $60 \%$. However, the two major sources of error are due to the parameterization of the employed algorithms and cannot challenge or question the syntactic approach in general. Using a context free grammar for assembly modeling, a semantic network for implementation, and discourse parsing strategies for detection thus provides a reliable solution for flexible assembly detection in an unconstrained environment.

The combined framework of syntactic and graph based methods investigated in this thesis constitutes a vision system that autonomously learns about individual assemblies. Parsing according to a context free grammar reliably detects arbitrary assembly structures in preprocessed image data. Assembly recognition is accomplished from matching the resulting mating feature graphs against a set of previously derived ones. Learning is realized by inserting those structures into that database which could not be matched. If this mechanism is integrated into a system for multi-modal man-machine interaction, a user may assign names to structures derived from an image. The system thus may learn about metonymies and what an assembly depicts and can acquire symbolic assembly knowledge far beyond the information a priori encoded in the grammar.

In conclusion, this thesis underlines that syntactic and graph based approaches are very well suited to treat various phenomena in cooperative assembly. Their application is based on sound theoretical concepts because mechanical assemblies show structural properties that are also investigated in the theories of formal languages, discourse, and semantics. In combination, syntactic and graph based methods provide a structural framework to describe and model different aspects of assemblies. Therefore, they can successfully be integrated into a system for man-machine cooperation in assembly. In particular, they provide an avenue to flexible and reliable assembly recognition from vision. 


\section{A. Further Notes on Mechanical Assemblies}

\section{A.1. Nonuniqueness of Context Free Assembly Structures}

On page 33, we presented a context free language that contains two words both characterizing an exemplary assembly of two bolts, a cube, and a bar with three holes. Strictly speaking, projecting a three-dimensional assembly onto a one-dimensional structure thus is not a mapping: a composite object may correspond to several words. Associating an assembly with a whole context free language, in contrast, is a mapping. However, we hinted that this mapping must not be one to one. A context free language may rather represent entire sets of different assemblies for there might be geometrically different configurations of a given collection of objects which all correspond to the same language. This becomes obvious from Fig. A.1.

Figure A.1 shows different configurations of the objects that form the assembly we used as an example. An AND/OR graph representation of the feasible decompositions of each of these assemblies will lead to the grammar we already know from the third chapter:

$$
\begin{aligned}
& \mathrm{A}_{1} \rightarrow B O L T_{2} \mathrm{~A}_{2} \mid B O L T_{1} B A R_{1} \mathrm{~A}_{3} \\
& \mathrm{~A}_{2} \rightarrow B O L T_{1} B A R_{1} C U B E_{1} \\
& \mathrm{~A}_{3} \rightarrow B O L T_{2} C U B E_{1} .
\end{aligned}
$$

Augmenting syntactic structures with details of mating relations between parts, however, leads to more unique descriptions. The high-level sequence plans we introduced on page 50 yield a different description for each configuration in the figure (see Fig. A.2).

\section{A.2. Mating Features Resulting from Assembly}

In our discussion on learning grammatical assembly models from examples, we noted that mating operations may create new types of mating features that are not contained in the set of elementary features provided by the underlying individual components. A simple assembly domain whose elementary parts are shown in Fig. A.3 will illustrate this. 


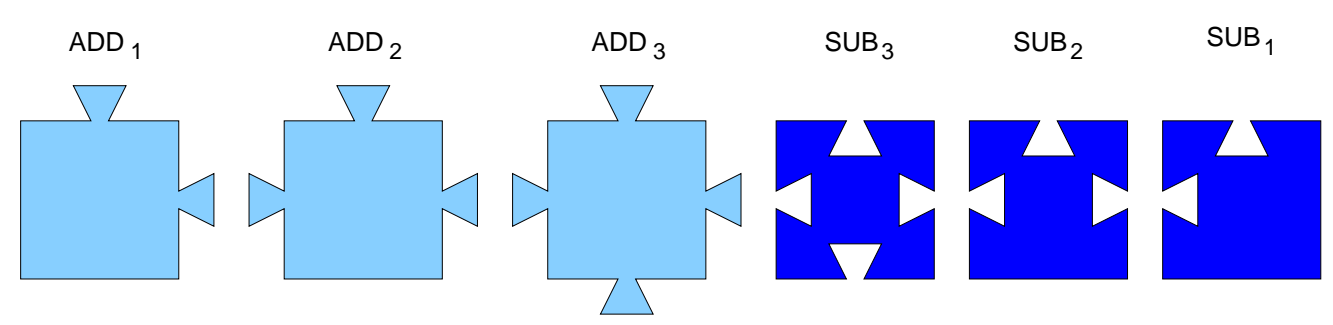

Figure A.3.: Elementary parts of a jigsaw puzzle. The set of elementary mating features provided by these parts is $\Phi=\{A D D, S U B\}$.
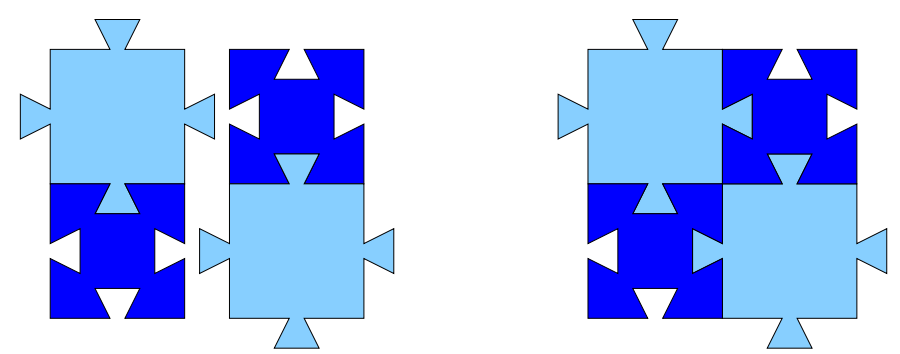

Figure A.4.: Assemblies of elementary pieces may show mating features of composite nature. These features may permit types of attachments that are not possible with elementary objects.

This grammar could, for example, represent the assembly on the right of Fig. A.4 by means of the following derivation:

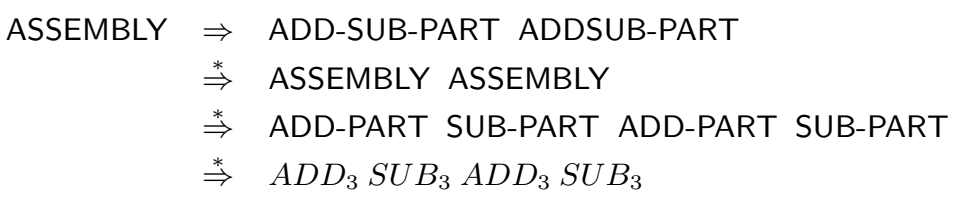

In Chapter 3, we pointed out that for any feasible assembly the set of mating features provided by its elementary parts is finite. Consequently, the number of composite features may be large but must be finite as well. Given these prerequisites, basic set theory yields a framework to reason about the mechanical function of subassemblies. If all elementary mating features of an assembly domain were collected in a set $\Phi$, any composite feature will correspond to a subset of $\Phi$. But the mechanical function of a part can be identified with the feature types it contributes to a connection. Thus, the function of a part can be mapped onto $2^{\Phi}$, the set of all subsets of $\Phi$. 


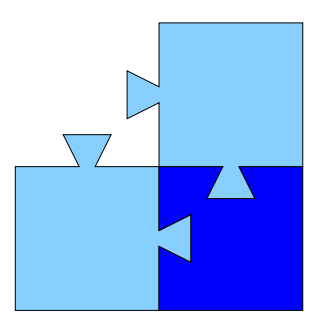

(a)

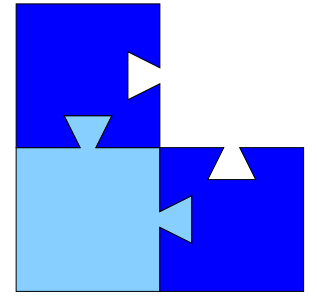

(b)

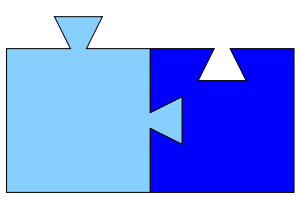

(c)

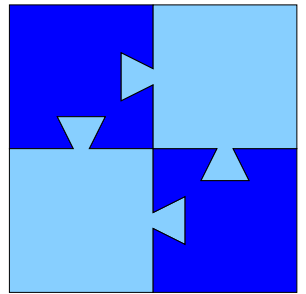

(d)

Figure A.5.: Simple puzzles assembled from some the parts introduced in Fig. A.3. Each assembly provides a (new) type of mating feature corresponding to a subset of $2^{\Phi}: \mathrm{A} .5(\mathrm{a}) \Leftrightarrow\{A D D\}, \mathrm{A} .5(\mathrm{~b}) \Leftrightarrow\{S U B\}, \mathrm{A} .5(\mathrm{c}) \Leftrightarrow\{A D D, S U B\}$, and A. $5(\mathrm{~d}) \Leftrightarrow \emptyset$

For the jigsaw domain we have $\Phi=\{A D D, S U B\}$ so that $2^{\Phi}=$ $\{\emptyset,\{A D D\},\{S U B\},\{A D D, S U B\}\}$. Figure A.5 shows that in this domain a corresponding assembly can be constructed for each element of the power set. Figures A.5(a) and A.5(b) depict examples of assemblies with additive and subtractive features, respectively. Figure A.5(c) shows an assembly that may serve as the ADD-SUB-PART of a more complex unit and Fig. A.5(d) underlines that, in general, it is even possible to construct assemblies with a function corresponding to $\emptyset$.

In most practical cases, however, the power set of the set of mating features will contain more than four elements and only few of the composite features will actually enable new kinds of attachments. In the baufix ${ }^{\circledR}$ domain, for instance, there are three basic mating features and of the eight combinatorial possible functions only five appear to be of practical relevance. If the elementary bolt, misc, and nut features are represented by the set $\Phi=\{B, M, N\}$, the functions possibly resulting from a mating operations correspond to $\{B\},\{M\},\{N\}, \emptyset$, and $\{M, N\}$. Exemplary realizations of the former three can be found in Chapter 3. Examples of the latter two are shown in Fig. A.6.

The assemblies in Fig. A.6(a) apparently cannot be used in further mating operations for they do not provide mating features. Figure A.6(b) shows an assembly with a composite feature. If the indicated mating operation was carried out, the assembly would contribute a feature of the misc type and a feature of the nut type to the connection. From this example we can see that the context free grammar for baufix ${ }^{\circledR}$ assemblies presented on page 31 should be extended by the productions:

$\begin{aligned} \text { ASSEMBLY } & \rightarrow \text { BOLT-PART MISC-NUT-PART } \\ \text { MISC-NUT-PART } & \rightarrow \text { ASSEMBLY }\end{aligned}$




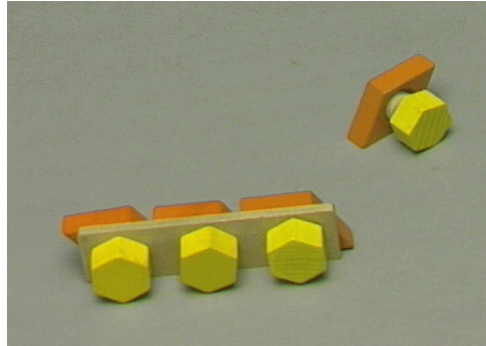

(a)

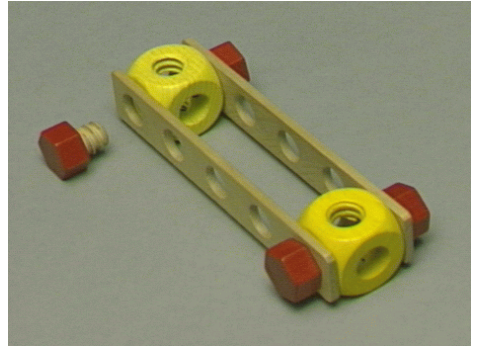

(b)

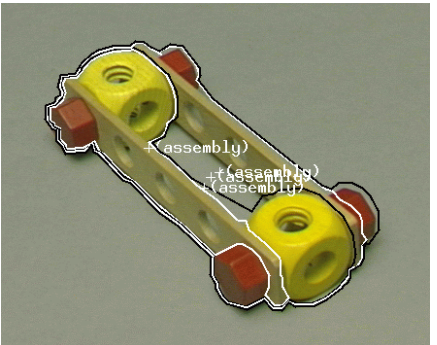

(c)

Figure A.6.: A.6(a) and A.6(b) Examples of baufix ${ }^{\circledR}$ assemblies with functions corresponding to $\emptyset$ and $\{M, N\}$, respectively. A.6(c) Detection result for an assembly containing a MISC-NUT-PART.

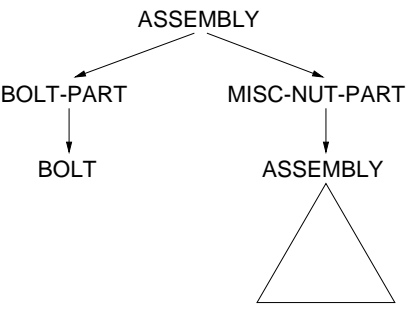

(a)

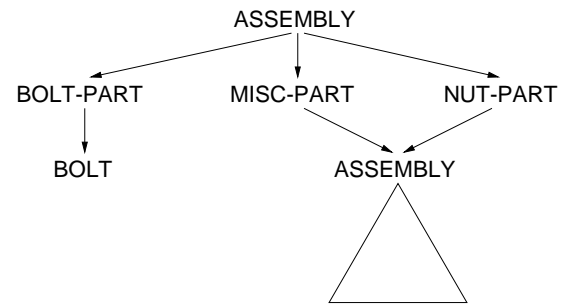

(b)

Figure A.7.: A.7(a) Theoretically desirable structure of the assembly in Fig. A.6(c). A.7(b) Solution realized in the ERNEST implementation of the assembly parser.

Since this functional relation between parts of an assembly could not be deduced from the examples presented in Chapter 3, it was not mentioned in the model presented there. Actually, it even is not realized as a modality with corresponding $\stackrel{\text { part }}{\longrightarrow}$ relations in our ERNEST implementation. Nevertheless, as Fig. A.6(c) proves, the flexibility offered by ERNEST enables our algorithm to cope with such assemblies and to detect them in image data.

In the implementation, all occurrences of a current assembly tree in an incoming assembly tree are unified to one singleton subtree. Figure A.7(a) shows the theoretically desirable structure and Fig. A.7(b) indicates how the assembly in Fig. A.6(c) is actually represented as an ERNEST instance tree. 
A. Further Notes on Mechanical Assemblies 


\section{B. Details on Assembly Detection from Parsing}

\section{B.1. An Example of Conjoining Assembly Trees}

In Chapter 3.2.2, we presented an algorithm for assembly detection that originated from ideas developed for discourse parsing. Here, we will further illustrate this approach and exemplify parsing by means of the assembly in Fig. B.2(a).

Remember that we assume labeled image regions resulting from an elementary object recognition procedure by Kummert et al. [73] as the grammatical primitives our approach deals with. Also remember that assembled objects are physically connected and therefore appear as clusters of classified regions once elementary object recognition is completed. Figure B.2(b) depicts the cluster that resulted for our example. Finally, recall that in order to detect assemblies by grammatical parsing the cluster elements have to be examined sequentially. But as pointed out earlier for baufix ${ }^{\circledR}$ assemblies sequencing is simple because objects attached to bolts are linearly arranged in the image plane.

Figures B.2(c)-B.5(c) show intermediate results and the final outcome of the assembly detection procedure. After starting at the yellow bolt, the process continues by examining the green cube. According to the grammatical model, bolt and cube form an assembly and the assembly tree $S U B_{1}$ in Fig. B.1(a) is derived. The corresponding screen display - a white polygon comprising both labeled regions - is shown in Fig. B.2(c). Since there still are unexamined objects in the cluster, the parsing process restarts at the red bolt. This leads to the detection of another subassembly $S U B_{2}$ with syntactic structure and screen display as shown in Fig. B.1(b) and Fig. B.2(d), respectively.

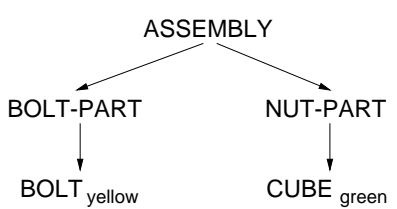

(a) $S U B_{1}$

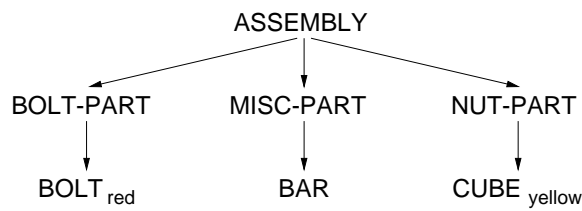

(b) $S U B_{2}$

Figure B.1.: Syntactic structures of the first two subassemblies found during parsing the cluster in Fig. B.2(b).

Continuing at the blue bolt yields assembly tree $S U B_{3}$ in Fig. B.3(a) and since blue bolts may have further objects attached to them, parsing directly proceeds with examin- 


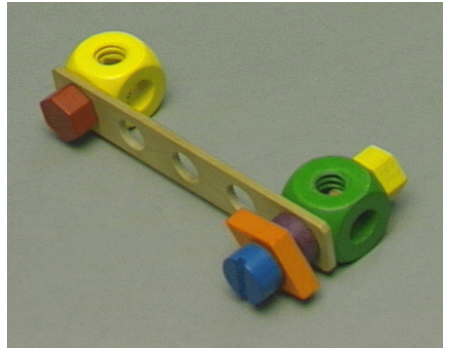

(a)

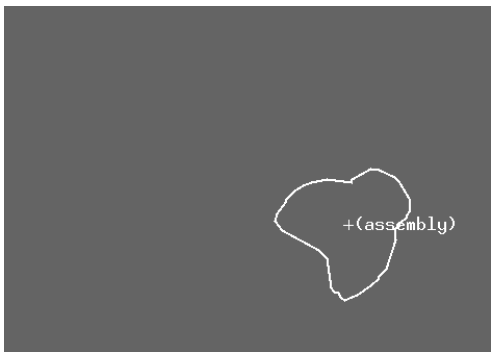

(c)

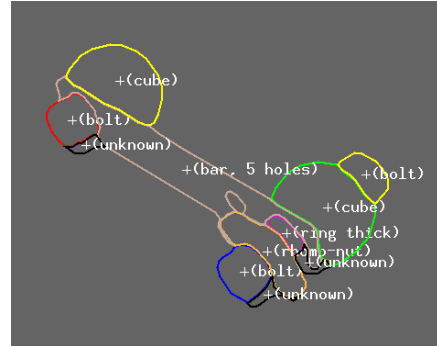

(b)

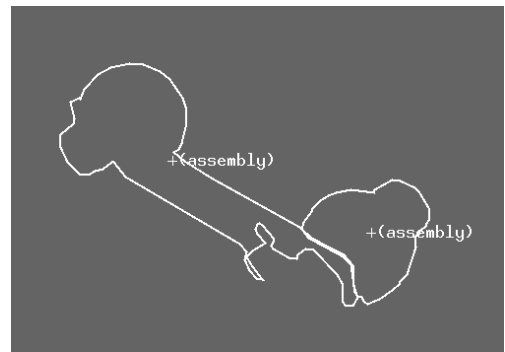

(d)

Figure B.2.: B.2(a), B.2(b) An exemplary assembly and the corresponding cluster of classified image regions. B.2(c) - B.5(b) Screen displays of the first two subassemblies found while parsing the cluster.

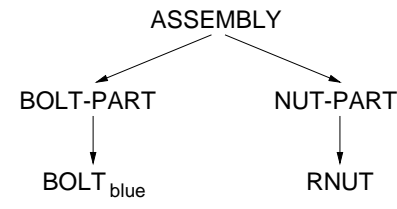

(a) $S U B_{3}$

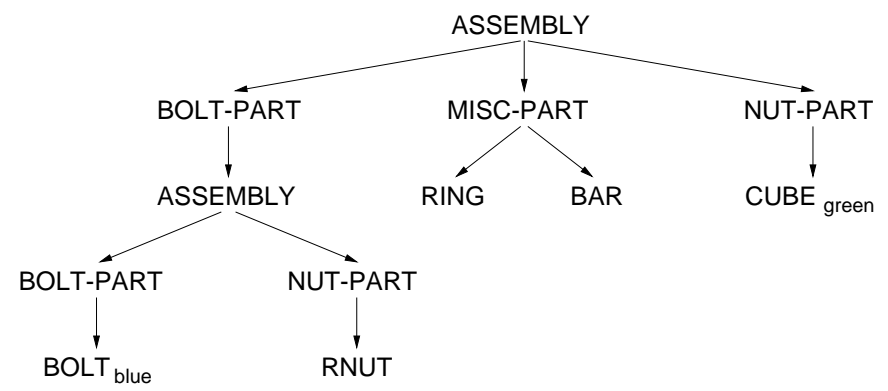

(b) $S U B_{4}$

Figure B.3.: Syntactic structures of two further subassemblies found during parsing the cluster in Fig. B.2(b).

ing the ring, the bar, and the green cube. This reveals another subassembly $S U B_{4}$ whose structure is shown in Fig. B.3(b). However, this current assembly tree shares an elemen- 


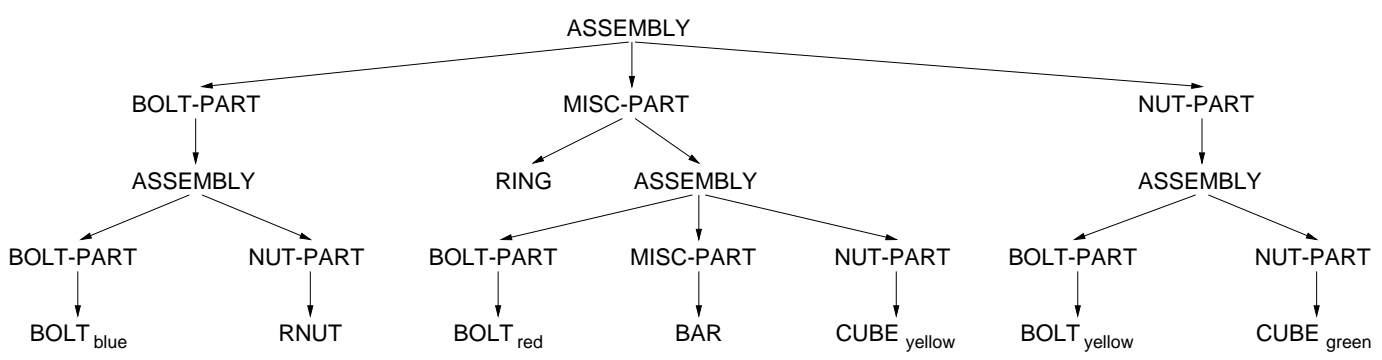

Figure B.4.: A possible syntactic structure of the whole assembly generated by conjoining the earlier found subassembly structures.

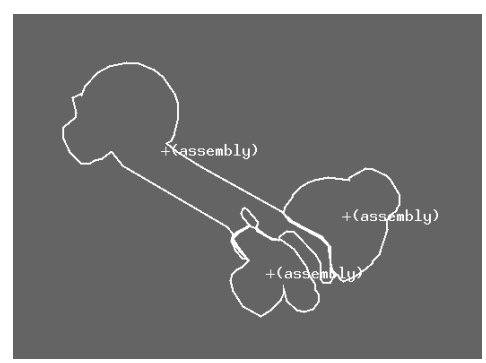

(a)

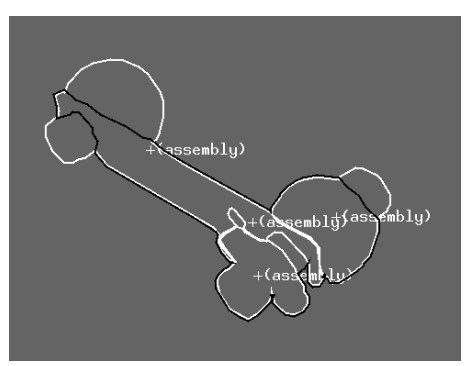

(b)

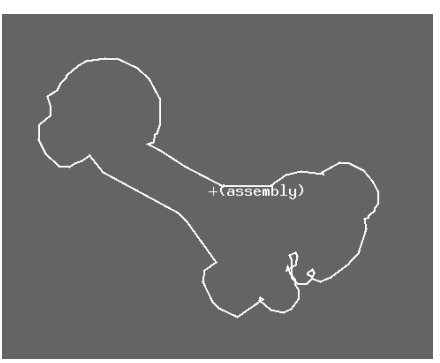

(c)

Figure B.5.: B.5(a) - B.5(b) Screen displays of further subassemblies found while parsing the cluster in Fig. B.2(b). B.5(c) Final result.

tary object with subassemblies $S U B_{1}$ and $S U B_{2}$. Consequently, all three descriptions are unified which results in the syntactic description $\left(S U B_{4} \triangle S U B_{2}\right) \triangle S U B_{1}$ in Fig. B.4. As this structure describes the whole assembly the polygon of the corresponding screen display in Fig. B.5(c) comprises all subassemblies found so far.

In Chapter 3.2.2, we also mentioned that there might be cases of current assemblies that share several objects with the incoming one. On the left of Fig. B.6, we see a corresponding example. The two bars arranged in parallel are part of any complex subassembly of the depicted assembly. Thus, no matter which subassembly will be detected first, it will always share two objects with the one detected next. The result in Fig. B.6(b) indicates that cases like this can be successfully coped with.

\section{B.2. An Example of Instantiating Assembly Concepts}

On page 42 in Chapter 3.2.1, we mentioned that using knowledge modeled in an ERNEST network is a process of creating and assessing modified concepts and instances and that 


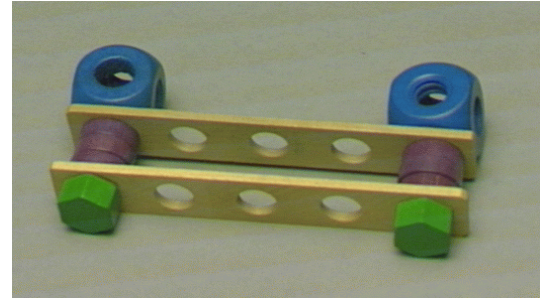

(a)

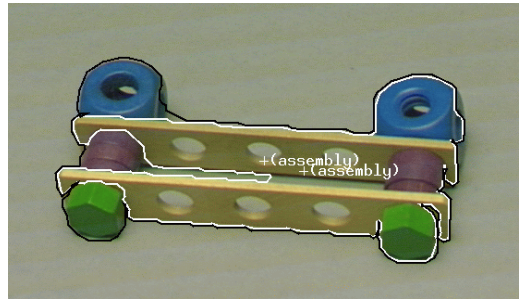

(b)

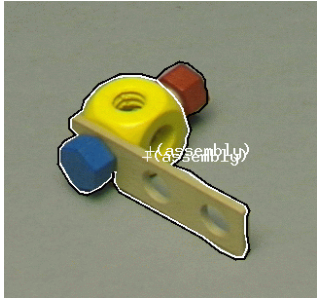

(c)

Figure B.6.: B.6(a), B.6(b) Example of an assembly containing subassemblies that are conjoined via several objects and corresponding detection result. B.6(c) Detection result for an assembly known from Chapter 3 .

an object is believed to be recognized as soon as instances for all its parts were generated. In the following, we will illustrate this by reconsidering the non discourse based parsing process that was described on page 43 .

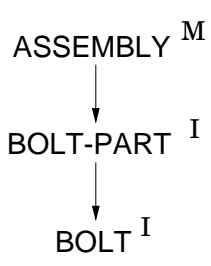

(a)

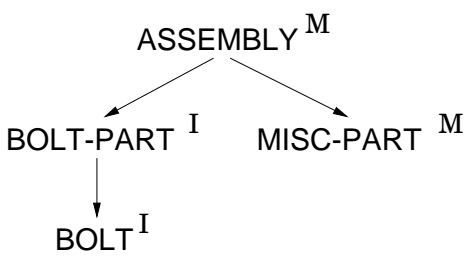

(b)

Figure B.7.: First stages of an instance tree generated while parsing the cluster of labeled regions corresponding to the assembly in Fig. B.6(c).

Figure B.6(c) once again shows the exemplary assembly we know from Chapter 3. The detection result cast into the image is the final outcome of a parsing process that, as it is based on an ERNEST implementation, involves the generation of an instance tree. Given instances of elementary objects, the instantiation of (sub)assembly concepts from parsing begins in a bottom-up manner. If the parsing process starts with a region representing a bolt, the first step is to link this bolt to a BOLT-PART concept. Consequently, the BOLTPART has a part and can immediately be instantiated. Next, the BOLT-PART is linked to an ASSEMBLY concept which can only become a modified concept for its other obligatory parts are still missing (see Fig. B.7(a)).

According to the structure of the exemplary cluster, parsing continues by considering the bar. As the bar is a miscellaneous object, a modified concept of MISC-PART is created 


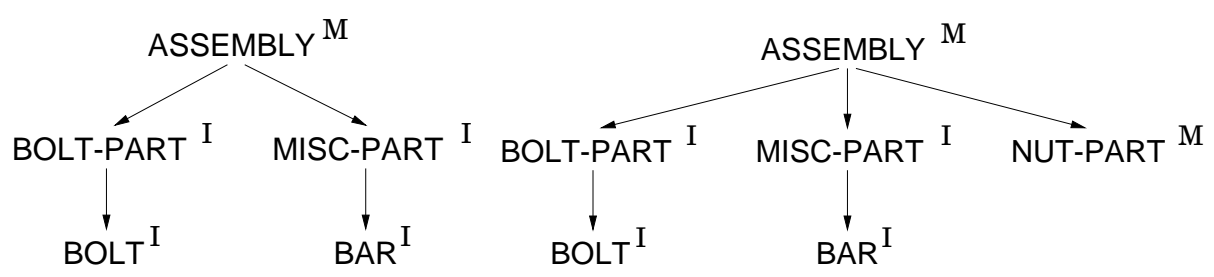

(a)

(b)

Figure B.8.: The modified concept of MISC-PART becomes an instance after the bar was linked to it and a top-down expansion of the ASSEMBLY is carried out.

in a top-down manner (see Fig. B.7(b)) and the corresponding instance of the bar will be linked to it.

This leads to the instantiation of the MISC-PART (see Fig. B.8(a)) because there are no further miscellaneous objects adjacent to the bar. Subsequently, a modified concept of NUT-PART is created and the instance representing the cube is linked to it. This causes the NUT-PART to be instantiated and also leads to the instantiation of the ASSEMBLY concept because now there are instances for all its obligatory parts (see Figs. B.8(b) and B.9(a)).

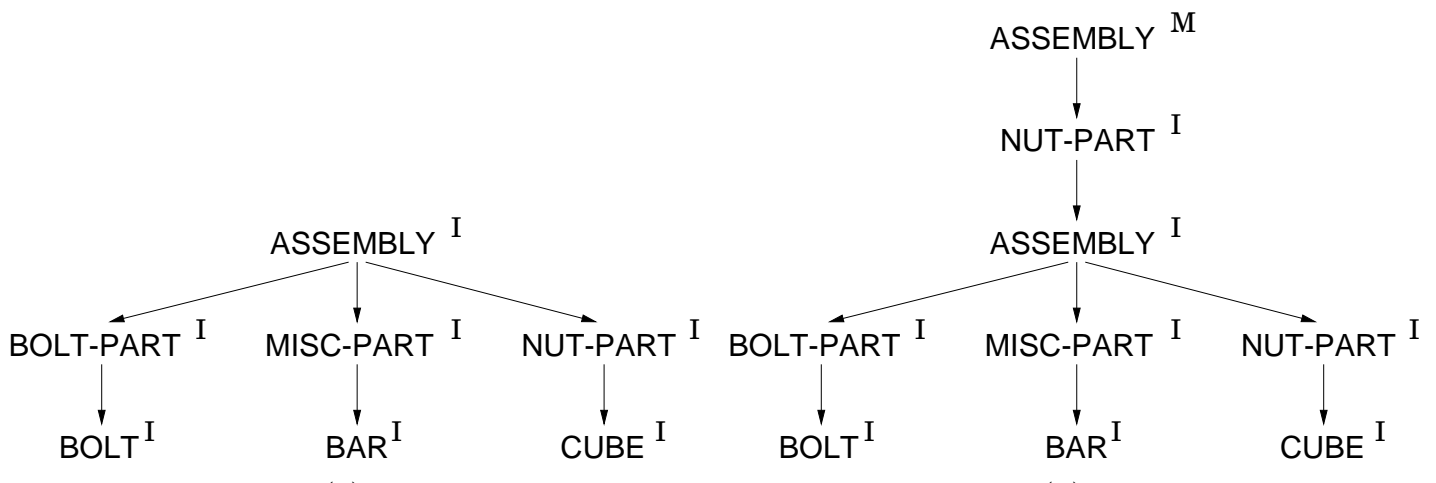

(a)

(b)

Figure B.9.: Instantiation of the ASSEMBLY concept and bottom-up expansion of the search tree corresponding to functional role of the just detected assembly.

At this stage of the analysis, the recently found assembly is hypothesized to be the NUT-PART of another assembly. Bottom-up expansion of the instance tree consequently yields a new instance of NUT-PART that is linked to another modified concept of ASSEMBLY (see Fig. B.9(b)).

Then, a modified concept of BOLT-PART is generated because there is no miscellaneous object adjacent to the cube so that the control algorithm automatically tries to generate 


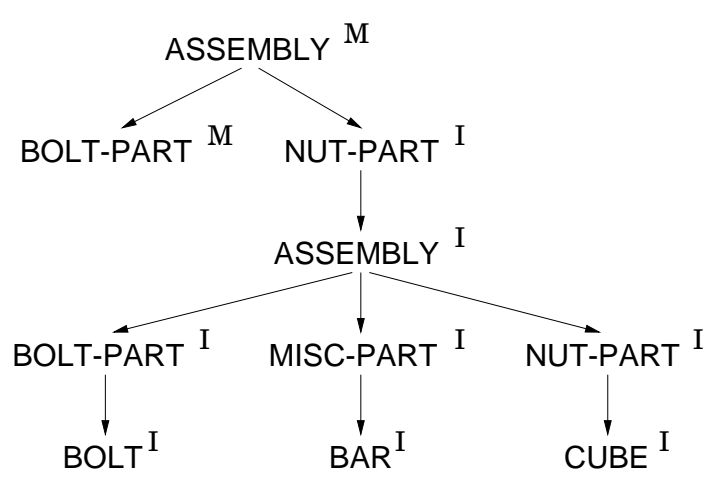

(a)

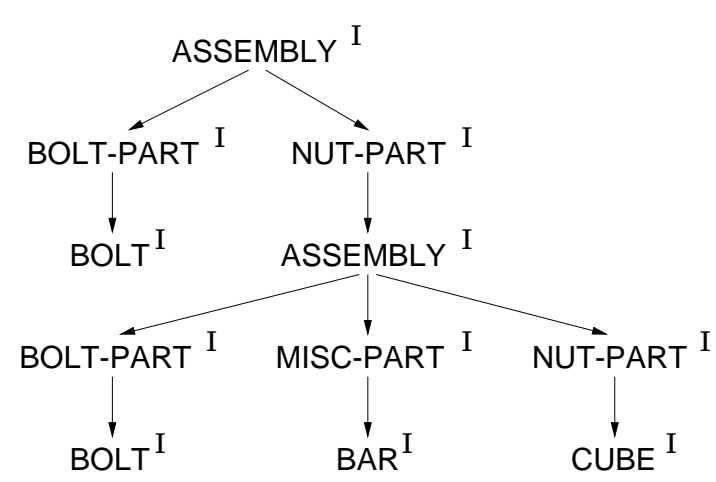

(b)

Figure B.10.: Top-down expansion of the modified ASSEMBLY concept and final state of the instance tree resulting from analyzing the object cluster.

instance of the other obligatory part of an ASSEMBLY (see Fig. B.10(a)). This succeeds because there actually is a bolt that can be linked to the BOLT-PART so that this can be instantiated. This, in turn, causes the instantiation of the ASSEMBLY concept (see Fig. B.10(b)). As there are no unexamined objects left, the parsing process terminates and the generated instance tree reflects the detected assembly structure. 


\section{Formal Proofs}

\section{C.1. ERnest and Context Free Grammars}

In Chapter 3.2.1, we claimed that a special kind of ERNEST networks is structurally equivalent to context free grammars. Here, we shall substantiate this proposition.

Modeled in a rather abstract mathematical way and neglecting many of the features ERNEST actually provides, an ERNEST knowledge base $K B$ is a 4 -tuple

$$
K B=(\boldsymbol{C}, \nu, \pi, \mu)
$$

where

$\boldsymbol{C}=\left\{C_{1}, C_{2}, \ldots, C_{n}\right\}$ is a set of concepts

$\nu: C \leadsto \boldsymbol{N A} \boldsymbol{M E}$ is a function assigning names to concepts.

$\pi: \boldsymbol{C} \leadsto \boldsymbol{P} \boldsymbol{R} \boldsymbol{T}={ }_{2} \boldsymbol{C}$ is a function assigning sets of parts to concepts.

$\mu: C \leadsto M O D$ is a function assigning sets of modalities to concepts.

If $C_{j} \in \pi\left(C_{i}\right) \subseteq \boldsymbol{C}$ we say that $C_{j}$ is a part of $C_{i}$ and usually write $C_{i} \stackrel{\text { part }}{\longrightarrow} C_{j}$. The set $M O D$ is a set of sets of modalities defined as

$$
\boldsymbol{M O D}=\left\{\left\{\left(\boldsymbol{P}_{i_{j}}, A_{i_{j}}\right) \mid j \in \mathbb{N}\right\} \mid i \in \mathbb{N}\right\}
$$

where each modality $\left(\boldsymbol{P}_{i_{j}}, A_{i_{j}}\right)$ consists of a multiset ${ }^{1} \boldsymbol{P}_{i_{j}}$ of concepts and a relation $A_{i_{j}} \subseteq \boldsymbol{P}_{i_{j}} \times \boldsymbol{P}_{i_{j}}$ called adjacency matrix.

For the special kind of network we will consider in the following, we require that given concepts $C^{1}, C^{2} \ldots, C^{m} \in \boldsymbol{P}_{i_{j}}, m=\left|\boldsymbol{P}_{i_{j}}\right|$ the adjacency matrix $A_{i_{j}}$ has the following form:

\begin{tabular}{c|cccccc} 
& $C^{1}$ & $C^{2}$ & $C^{3}$ & $\ldots$ & $C^{m-1}$ & $C^{m}$ \\
\hline$C^{1}$ & & 1 & & & & \\
$C^{2}$ & & & 1 & & & \\
$\vdots$ & & & & $\ddots$ & & \\
$C^{m-1}$ & & & & & & 1 \\
$C^{m}$ & & & & & &
\end{tabular}

\footnotetext{
${ }^{1} \mathrm{~A}$ multiset is a collection of objects that are not necessarily distinct [77]. For instance, $\{a, b, b, c, c, c\}$ is a multiset.
} 
Note that this actually defines a string of concepts and we may also write $A_{i_{j}}$ as:

$$
C^{1} \cdot C^{2} \cdot C^{3} \cdot \ldots \cdot C^{m}, C^{k} \in \boldsymbol{P}_{i_{j}}, m=\left|\boldsymbol{P}_{i_{j}}\right| .
$$

An ERNeST knowledge base $K B$ thus consists of concepts that may have parts. The parts are organized in modalities and each modality lists its collection of parts in a sequential order. Generally, a knowledge base $K B$ might be isomorphic to a context free grammar $G$ but does not have to be. However, consider the slightly extended definition:

Definition: An ERnEST knowledge base with root concept is a 5-tuple

$$
K B R=(\boldsymbol{C}, \nu, \pi, \mu, R)
$$

where $C, \nu, \pi, \mu$ are a set and functions as introduced above and $R \in C$ with $\pi(R) \neq \emptyset$ is called the root concept.

Based on this definition we can derive the following corrolar:

Corrolar: ERNEST knowledge bases with root concept are structurally equivalent to context free grammars.

Proof: We must show that for an arbitrary ERNEST knowledge base $K B R=$ $(\boldsymbol{C}, \nu, \pi, \mu, R)$ there is a corresponding context free grammar $G=(N, T, P, S)$. And that for an arbitrary context free grammar $G$ there is a corresponding ERNEST network $K B R$.

1. $K B R \Rightarrow G$ :

Choose the sets $N$ and $T$ of the grammar to be:

$$
\begin{aligned}
& N=\left\{\nu\left(C_{i}\right) \mid C_{i} \in C \wedge \pi\left(C_{i}\right) \neq \emptyset\right\} \\
& T=\left\{\nu\left(C_{i}\right) \mid C_{i} \in C \wedge \pi\left(C_{i}\right)=\emptyset\right\}
\end{aligned}
$$

The variables of the grammar thus correspond to the names of concepts that have parts, while terminals are given by names of concepts which do not have parts.

Set $S=\nu(R)$ for the start symbol of the grammar and define the productions $P$ as follows:

$$
P=\left\{p_{i_{j}}\left(C_{i}\right)\left|C_{i} \in C \wedge \pi\left(C_{i}\right) \neq \emptyset, j=1, \ldots l=\right| \mu\left(C_{i}\right) \mid\right\}
$$

with

$$
p_{i_{j}}\left(C_{i}\right)=\nu\left(C_{i}\right) \rightarrow \nu\left(C^{1}\right) \cdot \nu\left(C^{2}\right) \cdot \ldots \cdot \nu\left(C^{m}\right)
$$


where

$$
\left(\boldsymbol{P}_{i_{j}}, A_{i_{j}}\right) \in \mu\left(C_{i}\right), \quad C^{k} \in \boldsymbol{P}_{i_{j}}, \quad A_{i_{j}}=C^{1} \cdot C^{2} \cdot \ldots \cdot C^{m}, \quad m=\left|\boldsymbol{P}_{i_{j}}\right| .
$$

Hence, for each modality $\left(\boldsymbol{P}_{i_{j}}, A_{i_{j}}\right)$ of a concept $C_{i}$ in the knowledge base $K B R$ there is a grammatical production. Each of these productions relates a variable in $N$ and a sequence of symbols in $(N \cup T)^{*}$.

2. $G \Rightarrow K B R$ :

First, create a set of concepts $\boldsymbol{C}=\boldsymbol{C}_{T} \cup \boldsymbol{C}_{N}$ where

$$
\boldsymbol{C}_{T}=\{C(t) \mid t \in T\} \text { and } \boldsymbol{C}_{N}=\{C(n) \mid n \in N\}
$$

Thus, the set of concepts consists of two subsets where one contains concepts corresponding to the terminal symbols and the other contains concepts corresponding to the variables of $G$.

Choose the function $\nu$ as follows:

$$
\nu(C(a))=a, a \in N \cup T
$$

Hence, the names of concepts directly correspond to the names of variables and terminals.

Define the function $\pi$ as:

$$
\pi(C(a))=\left\{\begin{array}{cl}
\emptyset & \text { if } C(a) \in \boldsymbol{C}_{T} \\
\boldsymbol{P R T}(C(a)) & \text { if } C(a) \in \boldsymbol{C}_{N}
\end{array}\right.
$$

where

$$
\boldsymbol{P R T}(C(n))=\left\{C(a) \mid a \in N \cup T \wedge n \rightarrow u a v \in P \wedge u, v \in(N \cup T)^{*}\right\}
$$

Thus, for each symbol $a$ that appears on the right hand side of a production where the variable $n$ is on the left, the concept $C(a)$ becomes a part of the concept $C(n)$. Concepts corresponding to a terminal symbol do not have parts.

Next, define the function $\mu$ to be:

$$
\mu(C(a))=\left\{\begin{array}{cl}
\emptyset & \text { if } C(a) \in \boldsymbol{C}_{T} \\
\boldsymbol{M O D}(C(a)) & \text { if } C(a) \in \boldsymbol{C}_{N}
\end{array}\right.
$$

where

$$
\boldsymbol{M O D}(C(n))=\left\{(\boldsymbol{P}(w), A(w)) \mid n \rightarrow w \in P \wedge w \in(N \cup T)^{*}\right\}
$$


with

$$
\boldsymbol{P}\left(a_{1} \ldots a_{m}\right)=\left\{C\left(a_{1}\right), \ldots, C\left(a_{m}\right)\right\} \text { and } A\left(a_{1} \ldots a_{m}\right)=C\left(a_{1}\right) \cdot \ldots \cdot C\left(a_{m}\right)
$$

Hence, each sequence of symbols $a_{1} \ldots a_{m}$ that appears on the right hand side of a production with $n$ on the left hand side is transformed into a modality of the concept $C(n)$. The adjacency matrix of this modality reproduces the sequence of symbols. Concept corresponding to terminal symbols do not have any modality.

Finally, set $R=C(S) \in C$ where $S$ is the start symbol of $G$.

Thus, there is an isomorphism from $K B R$ to $G$ and vice versa. This proves the claim. $\square$

\section{C.2. Partial Orders}

In Chapter 3.3.1 the following definition of partially ordered sets was introduced:

Definition: A set $A$ is said to be partially ordered by a relation $\sqsubseteq$ if the following properties hold for every $x, y, z \in A$ :

1. $x \sqsubseteq x$.

2. $x \sqsubseteq y$ and $y \sqsubseteq x$ implies $x=y$.

3. $x \sqsubseteq y$ and $y \sqsubseteq z$ implies $x \sqsubseteq z$.

We also introduced a relation carries on $B O L T_{i}$, abbreviated $\sqsubseteq_{c_{i}}$, which holds between certain objects that are part of a bolted assemblies. It was formally defined as follows:

Definition: Let $o_{1}$ and $o_{2}$ be two elementary objects such that one of them is a bolt $B O L T_{i}$ and the other is attached to it, or, if none of them is a bolt, both are

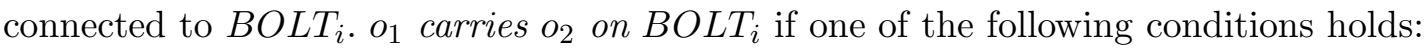

1. $o_{1}=o_{2}$, or

2. $o_{1}$ is $B O L T_{i}$ and $o_{2}$ is attached to it, or

3. $o_{1}$ was attached to $B O L T_{i}$ ere $o_{2}$ was attached to $B O L T_{i}$. 
Both definitions immediately lead to the following

Corrolar: With respect to the relation $\sqsubseteq_{c_{i}}$ a bolt $B O L T_{i}$ and a set of objects attached to it form a partially ordered set.

Proof: To proof the corrolar we must show that $\sqsubseteq_{c_{i}}$ matches the three properties that define a partial ordering relation.

1. Reflexivity

$o \sqsubseteq c_{i} o$ holds, if $o$ is $B O L T_{i}$ or an object attached to it, thus $\sqsubseteq_{c_{i}}$ is reflexive.

2. Antisymmetry

Case 1: $o_{1}=o_{2}$

$o_{1} \sqsubseteq_{c_{i}} o_{2}$ and $o_{2} \sqsubseteq_{c_{i}} o_{1}$ do hold.

Case 2: $o_{1} \neq o_{2}$

a) If w.l.o.g. $o_{1}$ is $B O L T_{i}$ and $o_{2}$ is an object attached to it, then $o_{1} \sqsubseteq_{c_{i}} o_{2}$ holds but $o_{2} \sqsubseteq_{c_{i}} o_{1}$ does not.

b) If w.l.o.g. $o_{1}$ and $o_{2}$ are attached to $B O L T_{i}$ where $o_{1}$ was attached earlier than $o_{2}$, then $o_{1} \sqsubseteq_{c_{i}} o_{2}$ holds but $o_{2} \sqsubseteq_{c_{i}} o_{1}$ does not.

Thus, $\sqsubseteq_{c_{i}}$ is antisymmetric.

3. Transitivity

Case 1: $o_{1}=o_{2}, o_{1}=o_{3}$, and $o_{2}=o_{3}$

$o_{1} \sqsubseteq_{c_{i}} o_{2}, o_{2} \sqsubseteq_{c_{i}} o_{3}$, and $o_{1} \sqsubseteq_{c_{i}} o_{3}$ do hold.

Case 1: $o_{1} \neq o_{2}, o_{1} \neq o_{3}$, and $o_{2} \neq o_{3}$

a) If w.l.o.g. $o_{1}$ is $B O L T_{i}$ and $o_{1} \sqsubseteq c_{i} o_{2}$ and $o_{2} \sqsubseteq c_{i} o_{3}$ then $o_{3}$ is attached to $B O L T_{i}$ and $o_{1} \sqsubseteq_{c_{i}} o_{3}$ holds.

b) If $o_{1}, o_{2}$, and $o_{3}$ are attached to $B O L T_{i}$ and w.l.o.g. $o_{1}$ was attached ere $o_{2}$ and $o_{2}$ was attached earlier than $o_{3}$, then $o_{1}$ was attached ere $o_{3}$, too. I.e. we have: $o_{1} \sqsubseteq c_{i} o_{2}, o_{2} \sqsubseteq c_{i} o_{3}$, and $o_{1} \sqsubseteq c_{i} o_{3}$.

Thus, $\sqsubseteq_{c_{i}}$ is transitive.

Therefore, $\sqsubseteq_{c_{i}}$ is a partial ordering relation.

And without considerable effort we can conclude another 
Corrolar: A set of objects $O$ that is partially ordered by $\sqsubseteq_{c_{i}}$ is a chain.

Proof: An object $o \in O$ either is $B O L T_{i}$ or it is attached to $B O L T_{i}$. If there were two objects $o_{1}, o_{2} \in O$ such that neither $o_{1} \sqsubseteq_{c_{i}} o_{2}$ nor $o_{2} \sqsubseteq_{c_{i}} o_{1}$, then if one of them was $B O L T_{i}$, the other one would not be attached to it. If none of them was $B O L T_{i}$, then due to the transitivity property of $\sqsubseteq_{c_{i}}$ at least one of them would not be attached to $B O L T_{i}$. Thus, by contradiction we see that for any two objects $o_{1}, o_{2} \in O$ either $o_{1} \sqsubseteq_{c_{i}} o_{2}$ or $o_{2} \sqsubseteq_{c_{i}} o_{1}$ must hold. Therefore, $O$ is a chain.

Finally, we shall motivate why the relation $\sqsubseteq_{c_{i}}$ was defined with respect to a certain bolt. Consider the following assembly and the corresponding partial order that would result if the ordering relation was not defined with respect to a bolt:
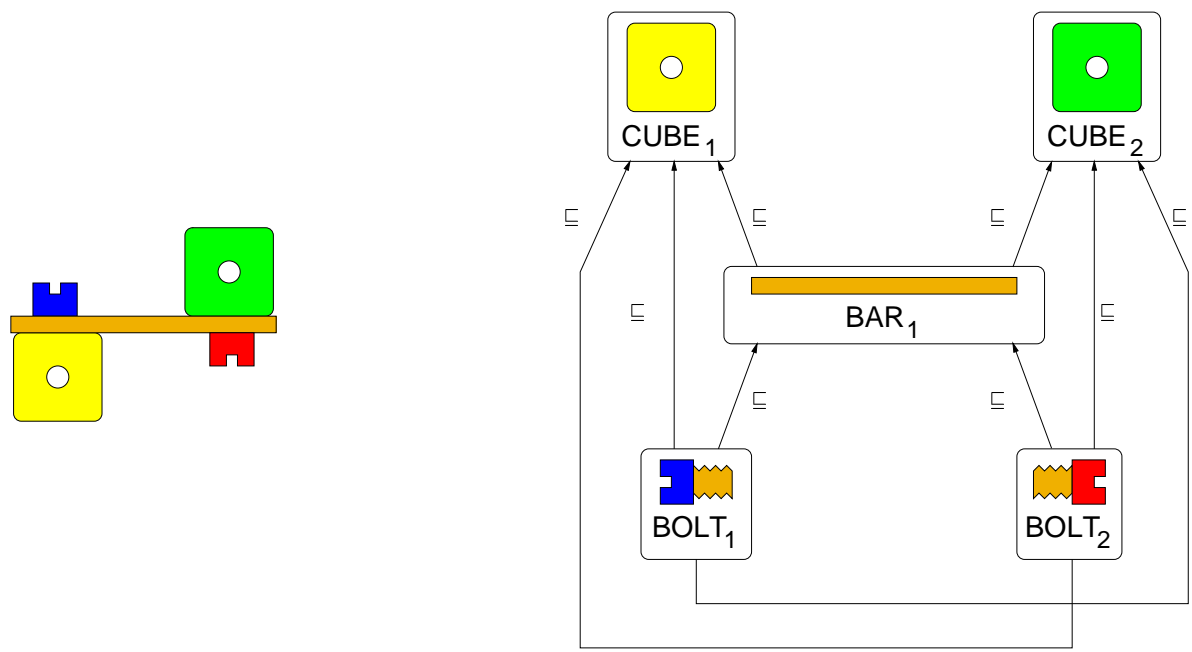

Of course, we would have two chains:

$$
B O L T_{1} \sqsubseteq B A R_{1} \sqsubseteq C U B E_{1} \text { and } B O L T_{2} \sqsubseteq B A R_{1} \sqsubseteq C U B E_{2} .
$$

But as $\sqsubseteq$ implies transitivity, we also had:

$$
B O L T_{1} \sqsubseteq C U B E_{2} \text { and } B O L T_{2} \sqsubseteq C U B E_{1} .
$$

Mathematically, this would be no problem; however, it seems less intuitive to relate bolts and cubes that are not attached to each other. 


\section{Complete Definition of the Semantics Function $\mathcal{S}$}

When we discussed the semantics of assembly sequence plans in Chapter 4.2.3, we introduced a denotational semantics function $\mathcal{S}$. However, only a few of the functions the calculation of $\mathcal{S}$ involves were presented in detail. The complete definition of the semantics function $\mathcal{S}$ and its subfunctions is presented in the following.

Many of the definitions listed below are known from Chapter 4.2.3 and do not have to be explained. The semantics of the objects FELLY, RING, and SOCKET correspond to the semantics of an RNUT which was explained on page 85. The semantics of a CUBE resembles the one of a BAR we know from pages 85-87. Nevertheless, there are subtle differences which shall be explained below.

$$
\begin{aligned}
& \mathcal{S}: \Sigma^{*} \leadsto M F G \\
& \mathcal{S} \llbracket p \rrbracket=\operatorname{partS} \llbracket p \rrbracket(\emptyset, \emptyset, \Omega, \Omega) \\
& \text { partS : } \Sigma^{*} \times M F G \sim M F G \\
& \operatorname{partS} \llbracket \operatorname{ASSEMBLY}(f) B M_{1} \ldots M_{n} N \rrbracket \Gamma=\operatorname{part} \mathcal{S} \llbracket N \rrbracket \ldots \operatorname{part} \mathcal{S} \llbracket M_{1} \rrbracket \text { partS } \llbracket B \rrbracket \Gamma \\
& \operatorname{partS} \llbracket \operatorname{BOLT} n \rrbracket \Gamma=\text { boltS } \llbracket \text { BOLT } n \rrbracket \Gamma \\
& \text { partS } \llbracket \text { FELLY } n H \rrbracket \Gamma=\text { fellyhole } \mathcal{S} \llbracket H \rrbracket n \Gamma \\
& \text { partS } \llbracket \text { RING } n H \rrbracket \Gamma=\text { ringhole } \mathcal{S} \llbracket H \rrbracket n \Gamma \\
& \text { partS } \llbracket \text { SOCKET } n H \rrbracket \Gamma=\text { sockethole } \mathcal{S} \llbracket H \rrbracket n \Gamma \\
& \text { partS } \llbracket \operatorname{BAR} n H_{1} \ldots H_{m} \rrbracket \Gamma= \\
& \text { interpartS } \llbracket \text { BAR } n \rrbracket \text { intrapartS } \llbracket \text { BAR } n \rrbracket \text { barhole } \mathcal{S} \llbracket H_{m} \rrbracket n m \ldots \text { barhole } \mathcal{S} \llbracket H_{1} \rrbracket n 1 \Gamma \\
& \text { part } \mathcal{S} \llbracket \operatorname{RNUT} n H \rrbracket \Gamma=\text { rnuthole } \mathcal{S} \llbracket H \rrbracket n \Gamma \\
& \text { part } \mathbb{S} \llbracket \operatorname{CUBE} n H_{1} \ldots H_{4} \rrbracket \Gamma= \\
& \text { interpart } \mathbb{S} \llbracket \text { CUBE } n \rrbracket \text { intrapart } \mathbb{S} \llbracket \text { CUBE } n \rrbracket \text { cubehole } \mathcal{S} \llbracket H_{4} \rrbracket n 4 \ldots \text { cubeholeS } \mathbb{S} \llbracket H_{1} \rrbracket n 1 \Gamma
\end{aligned}
$$


boltS : $\Sigma^{*} \times M F G \leadsto M F G$

boltS $\llbracket$ BOLT $n \rrbracket(V, E, \mu, \nu)=\left(V^{\prime}, E, \mu^{\prime}, \nu\right)$

$$
\begin{aligned}
V^{\prime} & =V \cup\left\{\operatorname{vrtx}\left(\mathrm{BOLT}_{n}\right)\right\} \\
\mu^{\prime} & =\mu\left[\frac{v r t x\left(\operatorname{BOLT}_{n}\right)}{\left(\mathrm{BOLT}_{1} \operatorname{HEAD}, \operatorname{col}\left(\mathrm{BOLT}_{n}\right)\right)}\right]
\end{aligned}
$$

fellyholeS : $\Sigma^{*} \times \mathbb{N} \times M F G \leadsto M F G$

fellyholeS $\llbracket($ BOLT $m) \rrbracket n(V, E, \mu, \nu)=\left(V^{\prime}, E^{\prime}, \mu^{\prime}, \nu^{\prime}\right)$

$$
\begin{aligned}
& V^{\prime}=V \cup\left\{v r t x\left(\text { FELLY }_{n}\right)\right\} \\
& E^{\prime}=E \cup\left\{\left(v, \operatorname{vrtx}\left(\operatorname{FELLY}_{n}\right)\right)\right\}, v \in V \wedge \operatorname{vrtx}\left(\operatorname{BOLT}_{m}\right) \sqsubseteq v \wedge \nexists v^{\prime} \in V: v \sqsubseteq v^{\prime} \\
& \mu^{\prime}=\mu\left[\frac{v r t x\left(\text { FELLY }_{n}\right)}{(\mathrm{FELLY}, \mathrm{HOLE}, \mathrm{red}}\right] \\
& \nu^{\prime}=\nu\left[\frac{\left(v, v r t x\left(\operatorname{FELLY}_{n}\right)\right)}{\sqsubseteq}\right]
\end{aligned}
$$

ringholeS : $\Sigma^{*} \times \mathbb{N} \times M F G \leadsto M F G$

ringhole $\mathcal{S} \llbracket($ BOLT $m) \rrbracket n(V, E, \mu, \nu)=\left(V^{\prime}, E^{\prime}, \mu^{\prime}, \nu^{\prime}\right)$

$$
\begin{aligned}
V^{\prime} & =V \cup\left\{v r t x\left(\mathrm{RING}_{n}\right)\right\} \\
E^{\prime} & =E \cup\left\{\left(v, \text { vrtx }\left(\mathrm{RING}_{n}\right)\right)\right\}, v \in V \wedge \operatorname{vrtx}\left(\mathrm{BOLT}_{m}\right) \sqsubseteq v \wedge \nexists v^{\prime} \in V: v \sqsubseteq v^{\prime} \\
\mu^{\prime} & =\mu\left[\frac{v r t x\left(\mathrm{RING}_{n}\right)}{\left(\operatorname{RING}, \mathrm{ROLE}_{\text {purpe }}\right.}\right] \\
\nu^{\prime} & =\nu\left[\frac{\left(v, v r t x\left(\mathrm{RING}_{n}\right)\right)}{\sqsubseteq}\right]
\end{aligned}
$$

sockektholeS : $\Sigma^{*} \times \mathbb{N} \times M F G \leadsto M F G$

sockekthole $\mathcal{S} \llbracket($ BOLT $m) \rrbracket n(V, E, \mu, \nu)=\left(V^{\prime}, E^{\prime}, \mu^{\prime}, \nu^{\prime}\right)$

$$
\begin{aligned}
& V^{\prime}=V \cup\left\{\operatorname{vrtx}\left(\operatorname{SOCKET}_{n}\right)\right\} \\
& E^{\prime}=E \cup\left\{\left(v, v r t x\left(\operatorname{SOCKET}_{n}\right)\right)\right\}, v \in V \wedge \operatorname{vrtx}\left(\operatorname{BOLT}_{m}\right) \sqsubseteq v \wedge \nexists v^{\prime} \in V: v \sqsubseteq v^{\prime} \\
& \mu^{\prime}=\mu\left[\frac{v \operatorname{vrtx}\left(\mathrm{SOCKET}_{n}\right)}{(\mathrm{SOCKT})}\right] \\
& \nu^{\prime}=\nu\left[\frac{\left(v, v r t x\left(\mathrm{RING}_{n}\right)\right)}{\sqsubseteq}\right]
\end{aligned}
$$

barholeS : $\Sigma^{*} \times \mathbb{N} \times \mathbb{N} \times M F G \leadsto M F G$

barhole $\mathcal{S} \llbracket() \rrbracket i j(V, E, \mu, \nu)=\left(V^{\prime}, E, \mu^{\prime}, \nu\right)$

$$
\begin{aligned}
V^{\prime} & =V \cup\left\{\operatorname{vrtx}\left(\mathrm{BAR}_{i j}\right)\right\} \\
\mu^{\prime} & =\mu\left[\frac{\operatorname{vrtx}\left(\mathrm{BAR}_{i j}\right)}{\text { (BAR,HOLE,wood } \left.)_{\text {BR }}\right)}\right.
\end{aligned}
$$




$$
\begin{aligned}
\text { barhole } & \mathcal{S} \llbracket(\operatorname{BOLT} m) \rrbracket i j(V, E, \mu, \nu)=\left(V^{\prime}, E^{\prime}, \mu^{\prime}, \nu^{\prime}\right) \\
V^{\prime} & =V \cup\left\{\operatorname{vrtx}\left(\mathrm{BAR}_{i j}\right)\right\} \\
E^{\prime} & =E \cup\left\{\left(v, \operatorname{vrtx}\left(\mathrm{BAR}_{i j}\right)\right)\right\}, v \in V \wedge \operatorname{vrtx}\left(\mathrm{BOLT}_{m}\right) \sqsubseteq v \wedge \nexists v^{\prime} \in V: v \sqsubseteq v^{\prime} \\
\mu^{\prime} & =\mu\left[\frac{v r t x\left(\mathrm{BAR}_{i j}\right)}{\left(\mathrm{BAR}_{\mathrm{HOLE}}, \mathrm{wood}\right)}\right] \\
\nu^{\prime} & =\nu\left[\frac{\left(v, v r t x\left(\mathrm{BAR}_{i j}\right)\right)}{\sqsubseteq}\right]
\end{aligned}
$$

rnutholeS : $\Sigma^{*} \times \mathbb{N} \times M F G \leadsto M F G$

rnuthole $\mathcal{S} \llbracket($ BOLT $m) \rrbracket n(V, E, \mu, \nu)=\left(V^{\prime}, E^{\prime}, \mu^{\prime}, \nu^{\prime}\right)$

$$
\begin{aligned}
V^{\prime} & =V \cup\left\{\operatorname{vrtx}\left(\operatorname{RNUT}_{n}\right)\right\} \\
E^{\prime} & =E \cup\left\{\left(v, \operatorname{vrtx}\left(\operatorname{RNUT}_{n}\right)\right)\right\}, v \in V \wedge \operatorname{vrtx}\left(\mathrm{BOLT}_{m}\right) \sqsubseteq v \wedge \nexists v^{\prime} \in V: v \sqsubseteq v^{\prime} \\
\mu^{\prime} & =\mu\left[\frac{\operatorname{vrtx}\left(\mathrm{RNUT}_{n}\right)}{\left(\mathrm{RNUT}_{1}, \mathrm{HOLE}_{\text {,orange }}\right)}\right] \\
\nu^{\prime} & =\nu\left[\frac{\left(v, \operatorname{vrtx}\left(\mathrm{RNUT}_{n}\right)\right)}{\sqsubseteq}\right]
\end{aligned}
$$

cubeholeS : $\Sigma^{*} \times \mathbb{N} \times \mathbb{N} \times M F G \leadsto M F G$

cubeholeS $\llbracket() \rrbracket i j(V, E, \mu, \nu)=\left(V^{\prime}, E, \mu^{\prime}, \nu\right)$

$$
\begin{aligned}
V^{\prime} & =V \cup\left\{\operatorname{vrtx}\left(\operatorname{CUBE}_{i j}\right)\right\} \\
\mu^{\prime} & =\mu\left[\frac{\operatorname{vrtx}\left(\operatorname{CUBE}_{i j}\right)}{\left(\operatorname{CUBE}_{1}, \mathrm{HOLE}, \operatorname{col}\left(\mathrm{CUBE}_{i}\right)\right)}\right]
\end{aligned}
$$

cubeholeS $\llbracket($ BOLT $m) \rrbracket i j(V, E, \mu, \nu)=\left(V^{\prime}, E^{\prime}, \mu^{\prime}, \nu^{\prime}\right)$

$$
\begin{aligned}
V^{\prime} & =V \cup\left\{\operatorname{vrtx}\left(\operatorname{CUBE}_{i j}\right)\right\} \\
E^{\prime} & =E \cup\left\{\left(v, \operatorname{vrtx}\left(\operatorname{CUBE}_{i j}\right)\right)\right\}, v \in V \wedge \operatorname{vrtx}\left(\operatorname{BOLT}_{m}\right) \sqsubseteq v \wedge \nexists v^{\prime} \in V: v \sqsubseteq v^{\prime} \\
\mu^{\prime} & =\mu\left[\frac{v r t x\left(\operatorname{CUBE}_{i j}\right)}{\left.\left(\operatorname{CUBE}_{i}, \operatorname{HOLE}, \operatorname{col}_{(\operatorname{CUBE}}\right)\right)}\right] \\
\nu^{\prime} & =\nu\left[\frac{\left(v, v r t x\left(\mathrm{CUBE}_{i j}\right)\right)}{\sqsubseteq}\right]
\end{aligned}
$$

intrapartS : $\Sigma^{*} \times M F G \leadsto M F G$

intrapartS $\llbracket \operatorname{BAR} n \rrbracket(V, E, \mu, \nu)=\left(V, E^{\prime}, \mu, \nu^{\prime}\right)$

$$
\begin{aligned}
E^{\prime} & =E \cup E_{\text {intra }} \\
\nu^{\prime} & =\nu\left[\frac{e}{\text { ADJ }}\right], \forall e \in E_{\text {intra }} \\
V_{B_{n}} & =\left\{v r t x\left(\mathrm{BAR}_{i j}\right) \in V \mid i=n \wedge j \in \mathbb{N}\right\} \\
E_{\text {intra }} & =\left\{\left(v_{i}, v_{i+1}\right),\left(v_{i+1}, v_{i}\right)\left|v_{i}, v_{i+1} \in V_{B_{n}} \wedge i=1, \ldots,\right| V_{B_{n}} \mid-1\right\}
\end{aligned}
$$

intrapartS $\llbracket \operatorname{CUBE} n \rrbracket(V, E, \mu, \nu)=\left(V^{\prime}, E^{\prime}, \mu^{\prime}, \nu^{\prime}\right)$ 


$$
\begin{aligned}
& V^{\prime}=V \cup\left\{v r t x\left(\operatorname{CUBE}_{n}\right)\right\} \\
& E^{\prime}=E \cup E_{\text {intra }} \\
& \mu^{\prime}=\mu\left[\frac{v r t x\left(\operatorname{CUBE}_{n}\right)}{\left(\operatorname{CUBE}, \mathrm{CENTER}, \operatorname{col}\left(\mathrm{CUBE}_{n}\right)\right)}\right] \\
& \nu^{\prime}=\nu\left[\frac{e}{\mathrm{ADJ}}\right], \forall e \in E_{\text {intra }} \\
& V_{C_{n}}=\left\{\operatorname{vrtx}\left(\mathrm{CUBE}_{i j}\right) \in V \mid i=n \wedge j \in \mathbb{N}\right\} \\
& E_{\text {intra }}=\left\{\left(v, \text { vrtx }\left(\mathrm{CUBE}_{n}\right)\right),\left(\text { vrtx }\left(\operatorname{CUBE}_{n}\right), v\right) \mid v \in V_{C_{n}}\right\}
\end{aligned}
$$

Applied to a CUBE, the function intrapartS relates the vertices representing the corresponding cube holes. However, as explained in Chapter 4.2.2, we regard the center of a CUBE as a virtual mating feature. Thus, first intrapart $\mathcal{S}$ creates a vertex representing the center. Then, the vertices representing holes are linked to it where the corresponding edges are labeled 'ADJ'.

$$
\begin{aligned}
& \text { interpartS }: \Sigma^{*} \times M F G \leadsto M F G \\
& \begin{aligned}
\text { interpartS } \llbracket \operatorname{BAR} n H_{1} \ldots H_{m} \rrbracket(V, E, \mu, \nu)=\left(V, E^{\prime}, \mu, \nu^{\prime}\right) \\
E^{\prime}=E \cup\left\{e \mid\left(e,\left(s, s^{\prime}\right)\right) \in P_{2}\right\} \\
\nu^{\prime}=\nu\left[\frac{e}{\varphi\left(s, s^{\prime}\right)}\right], \forall\left(e,\left(s, s^{\prime}\right)\right) \in P_{2} \\
P_{1}=\left\{\left(v r t x\left(\mathrm{BOLT}_{m}\right), s\right) \mid \text { vrtx }\left(\mathrm{BOLT}_{m}\right) \in V \wedge\left(\mathrm{BOLT}_{m}, s\right) \in\left\{H_{1}, \ldots, H_{m}\right\}\right\} \\
P_{2}=\left\{\left(\left(v, v^{\prime}\right),\left(s, s^{\prime}\right)\right) \mid(v, s),\left(v^{\prime}, s^{\prime}\right) \in P_{1} \wedge v \neq v^{\prime}\right\}
\end{aligned}
\end{aligned}
$$

where

$$
\begin{aligned}
& \varphi\left(s, s^{\prime}\right)= \begin{cases}0^{\circ}, & \text { if } s=s^{\prime} \\
180^{\circ}, & \text { otherwise. }\end{cases} \\
& \text { interpartS } \llbracket \text { CUBE } n H_{1} \ldots H_{4} \rrbracket(V, E, \mu, \nu)=\left(V, E^{\prime}, \mu, \nu^{\prime}\right) \\
& E^{\prime}=E \cup\left\{e \mid\left(e,\left(i, i^{\prime}\right)\right) \in P_{2}\right\} \\
& \nu^{\prime}=\nu\left[\frac{e}{\psi\left(i, i^{\prime}\right)}\right], \forall\left(e,\left(i, i^{\prime}\right)\right) \in P_{2} \\
& P_{1}=\left\{\left(v r t x\left(\mathrm{BOLT}_{m}\right), i\right) \mid \text { vrtx }\left(\mathrm{BOLT}_{m}\right) \in V \wedge H_{i}=\left(\mathrm{BOLT}_{m}\right) \in\left\{H_{1}, \ldots, H_{4}\right\}\right\} \\
& P_{2}=\left\{\left(\left(v, v^{\prime}\right),\left(i, i^{\prime}\right)\right) \mid(v, i),\left(v^{\prime}, i^{\prime}\right) \in P_{1} \wedge v \neq v^{\prime}\right\}
\end{aligned}
$$

where

$$
\psi\left(i, i^{\prime}\right)= \begin{cases}90^{\circ}, & \text { if }\left|i-i^{\prime}\right| \bmod 2=1 \\ 180^{\circ}, & \text { otherwise }\end{cases}
$$

To compute the relations between bolts attached to the same CUBE, the vertices representing those bolts are paired with an index indicating the number of the hole they are attached to. These pairs are collected in the set $P_{1}$. Then each two pairs from $P_{1}$ are transformed into a tuple whose first component contains two vertices (i.e. an edge) 
while the second component contains the corresponding indices. These form the set $P_{2}$ where the angle between bolts is computed from: for each element in $P_{2}$ the edge in the first component is labeled with the result of a function $\psi$ applied to the indices from the second component. For instance, a bolt in hole 1 encloses an angle of $90^{\circ}$ with a bolt in hole 2 or in hole 4 while it will face a bolt in hole 3 so that the angle would be $180^{\circ}$. 
D. Complete Definition of the Semantics Function $\mathcal{S}$ 


\section{Bibliography}

[1] A. Abella and J. R. Kender. Qualitatively describing objects using spatial prepositions. In Proc. AAAI'93, pages 536-540, 1993.

[2] M.G. Abu-Hamdan and A. Sherif-El-Gizawy. Computer-aided monitoring system for flexible assembly operations. Computers In Industry, 34(1):1-10, 1997.

[3] A.P. Ambler, H.G Barrow, C.M. Brown, R.M. Burstall, and R.J. Popplestone. A Versatile System for Computer Controlled Assembly. Artificial Intelligence, 6(2):129-156, 1975.

[4] A.P. Ambler and R.J. Popplestone. Inferring the Position of Bodies from Specified Spatial Relationships. Artificial Intelligence, 6:157-174, 1975.

[5] R. Anantha, G.A. Kramer, and R.H Crawford. Assembly modelling by geometric constraint satisfaction. Computer-Aided Design, 28(9):707-722, 1996.

[6] F. Arbab. Set Models and Boolean Operations for Solids and Assemblies. IEEE Computer Graphics \& Applications, pages 76-86, November 1990.

[7] C. Bauckhage, G.A. Fink, J. Fritsch, F. Kummert, F. Lömker, G. Sagerer, and S. Wachsmuth. An Integrated System for Cooperative Man-Machine Interaction. In Proc. IEEE International Symposium on Computational Intelligence in Robotics and Automation (CIRA'2001), 2001.

[8] C. Bauckhage, J. Fritsch, F. Kummert, and G. Sagerer. Towards a Vision System for Supervising Assembly Processes. In Proc. Symposium on Intelligent Robotic Systems (SIRS'99), pages 89-98, 1999.

[9] C. Bauckhage, J. Fritsch, and G. Sagerer. Erkennung von Aggregaten aus Struktur und Handlung. Künstliche Intelligenz, 3:4-11, 1999.

[10] C. Bauckhage, J. Fritsch, and G. Sagerer. Memorizing Visual Knowledge for Assembly Process Monitoring. In B. Radig and S. Florczyk, editors, Pattern Recognition, volume 2191 of Lecture Notes in Computer Science, pages 178-185. SpringerVerlag, 2001. 
[11] C. Bauckhage, S. Kronenberg, F. Kummert, and G. Sagerer. Grammars and Discourse Theory to Describe and Recognize Mechanical Assemblies. In Advances in Pattern Recognition, volume 1876 of Lecture Notes in Computer Science, pages 173-182. Springer-Verlag, 2000.

[12] C. Bauckhage, F. Kummert, and G. Sagerer. Modeling and Recognition of Assembled Objects. In Proc. IEEE IECON'98, pages 2051-2056, 1998.

[13] C. Bauckhage, F. Kummert, and G. Sagerer. Learning Assembly Sequence Plans Using Functional Models. In Proc. IEEE International Symposium on Assembly and Task Planning (ISATP'99), pages 1-7, 1999.

[14] C. Bauckhage, H. Rieser, B. Jung, , and I. Voss. Struktur, Semiotik und Dynamik - ein Überblick über Aspekte und Methoden der Modellierung mechanischer Aggregate. Report 1, SFB 360 'Situierte Künstliche Kommunikatoren', Universität Bielefeld, 2001.

[15] C. Bauckhage, S. Wachsmuth, and G. Sagerer. 3D Assembly Recognition by Matching Functional Subparts. In Proc. 3rd IAPR-TC15 Workshop on Graph-based Representations in Pattern Recognition (GbR 2001), pages 95-104, 2001.

[16] M. Bertolotto, E. Bruzzone, L. De Floriani, and G. Nagy. Generating assembly and machining sequences from the Face-to-Face Composition model. Computer-Aided Design, 28(2):101-112, 1996.

[17] A. Bourjault. Contribution à une approche méthodologique de l'Assemblage Automatisé: Elaboration Automatique des Séquences Opératoires. $\mathrm{PhD}$ thesis, Université de Franche-Comté, 1984.

[18] E. Braun, G. Heidemann, H. Ritter, and G. Sagerer. A multi-directional multile path recognition scheme for complex objects applied to the domain of a wooden toy kit. Pattern Recognition Letters, 20:1085-1091, 1999.

[19] K.N. Brown, C.A. McMahon, and J.H. Sims Williams. Describing process plans as the formal semantics of a language of shape. Artificial Intelligence in Engineering, 10(2), 1996.

[20] H. Bunke. Modellgesteuerte Bildanalyse. B.G. Teubner, Stuttgart, 1985.

[21] H. Bunke. Graph matching for visual object recognition. Spatial Vision, 13(23):335-340, 2000.

[22] H. Bunke and B.T. Messmer. Recent Advances in Graph Matching. International Journal of Pattern Recognition and Artificial Intelligence, 11(1):169-203, 1997. 
[23] R. Caracciolo, E. Ceresole, T. De Martino, and F. Giannini. From CAD Models to Assembly Planning. In W. Straßer and F.M. Wahl, editors, Graphics and Robotics, pages 115-129. Springer-Verlag, Berlin, 1995.

[24] S. Chakrabarty and J. Wolter. A Structure-Oriented Approach to Assembly Sequence Planning. IEEE Transactions on Robotics and Automation, 13(1):14-29, 1997.

[25] K.H. Chang and W.G. Wee. A knowledge-based planning system for mechanical assembly using robots. IEEE Expert, 3(1):18-30, 1988.

[26] K. Chen and J.-M. Henrioud. Systematic Generation of Assembly Precedence Graphs. In Proc. IEEE Int. Conf. Robotics and Automation, pages 1476-1482, 1994.

[27] N. Chomsky. Syntactic Structures. Mouton and Company, The Hague, 1957.

[28] T.L. De Fazio, S.J. Rhee, and D.E. Whitney. Design-Specific Approach to Design fo Assembly (DFA) for Complex Mechanical Assemblies. IEEE Transactions on Robotics and Automation, 15(5):869-881, 1999.

[29] L. De Floriani, A. Maulik, and G. Nagy. Representation of solid objects by a modular boundary model. In L.S. Homem de Mello and S. Lee, editors, Computeraided mechanical assembly planning, pages 41-90. Kluwer Academic Press, 1991.

[30] P. Deransart, M. Jourdan, and B. Loho, editors. Attributed Grammars, volume 323 of Lecture Notes in Computer Science. Springer, 1988.

[31] M. Ehrenmann, R. Zöllner, S. Knoop, and R. Dillmann. Sensor Fusion Approaches for Obbservation of User Actions in Programming by Demonstration. In Proc. Int. Conf. on Multisensor Fusion and Integration for Intelligent Systems, pages 227$232,2001$.

[32] T.-H. Eng, Z.-K. Ling, W. Olson, and C. McLean. Feature-based assembly modeling and sequence generation. Computers \& Industrial Engineering, 36(1):17-33, 1999.

[33] N. Esau and L. Steinborn. Konzeption und Realisierung eines Aggregatgedächtnisses zur Analyse von Konstruktionsprozessen. Master Thesis, Bielefeld University, Feb. 2001. available at: www.TechFak.Uni-Bielefeld.DE/techfak/ ags/ai/publications/master-theses/Esau_Steinborm2001.ps.gz.

[34] O. Faugeras. Three-Dimensional Computer Vision. MIT Press, Cambridge, MA, 1993. 
[35] J. Feder. Plex Languages. Information Sciences, 3:225-241, 1971.

[36] E. Fehr. Semantik von Programmiersprachen. Springer-Verlag, Berlin, 1989.

[37] E. Freund and J. Rossmann. Projective Virtual Reality: Bridging the Gap between Virtual Reality and Robotics. IEEE Transactions on Robotics and Automation, 15(3):411-422, 1999.

[38] K. Fries, J. Meyer, B. Lindemann, and H. Hagen. Correspondence Analysis: The Matching of 3-Dimensional Sets of Points. In Proc. Vision, Modelling, and Visualization 2000, pages 399-406. infix, 2000.

[39] K.S. Fu, editor. Digital Pattern Recognition, chapter 4. Springer-Verlag, Berlin, 1976.

[40] K.S. Fu, editor. Syntactic Pattern Recognition, Applications. Springer-Verlag, Berlin, 1977.

[41] J.Y.H. Fuh, C.-H. Chang, and M.A. Melkanoff. The development of an integrated and intelligent $\mathrm{CAD} / \mathrm{CAPP} / \mathrm{CAFP}$ environment using logic-based reasoning. Computer-Aided Design, 28(3):217-232, 1996.

[42] T. Fuhr, G. Socher, C. Scheering, and G. Sagerer. A three-dimensional spatial model for the interpretation of image data. In IJCAI-95 Workshop on Representation and Processing of Spatial Expressions, Montreal, Canada, 1995. $14^{t} h$ Int. Joint Conf. on Art. Int. (IJCAI-95).

[43] G. Dudek and M. Jenkin. Computational Principles of Mobile Robotics. Cambridge University Press, New York, 2000.

[44] C. Gardent. Discourse tree adjoining grammar. Technical report, University of Saarland, CLAUS report 91, 1997.

[45] S. Ginsburg. The Mathematical Theory of Context-Free Languages. McGraw-Hill, New York, 1966.

[46] M. Goldwasser, J.-C. Latombe, and R. Motwani. Complexity Measures for Assembly Sequences. In Proc. IEEE Int. Conf. Robotics and Automation, pages 1581-1587, 1996.

[47] J. González-Linares, N. Guil, P. Pérez, and R. Dillmann. An Efficient Image Processing Algorithm for High-Level Skill Acquisition. In Proc. IEEE International Symposium on Assembly and Task Planning (ISATP'99), pages 262-267, 1999.

[48] J.-K. Gui and M. Mäntylä. Functional Understanding of Assembly Modelling. Computer-Aided Design, 28(6):435-451, June 1994. 
[49] P.A.V. Hall. Equivalence Between AND/OR Graphs and Context-Free Grammars. Communications of the ACM, 16(7):444-445, 1973.

[50] M. Hanheide. Objektbezogene 3D-Erkennung automatisch generierter Konturmodelle in Intensitätsbildern. Master Thesis (Diplomarbeit), Bielefeld University, 2001. available at: www.TechFak.Uni-Bielefeld.DE/techfak/ags/ai/ publications/master-theses/Hanheide2001.ps.gz.

[51] J.-M. Henrioud and A. Bourjault. LEGA: a computer-aided generator of assembly plans. In L.S. Homem de Mello and S. Lee, editor, Computer-aided mechanical assembly planning, pages 191-215. Kluwer Academic Publishers, 1991.

[52] M. Hoffhenke and I. Wachsmuth. Object Recognition with Shape Prototypes in a 3D Construction Scenario. In W. Burgard, T. Christaller, and A. B. Cremers, editors, KI-99: Advances in Artificial Intelligence, volume 1701 of Lecture Notes in Artificial Intelligence, pages 231-242. Springer-Verlag, 1999.

[53] L.S. Homem de Mello and A.C. Sanderson. A correct and complete algorithm for the generation of mechanical assembly sequences. In Proc. IEEE Int. Conf. Robotics and Automation, pages 56-61, 1989.

[54] L.S. Homem de Mello and A.C. Sanderson. Representations of assembly sequences. In Proc. IEEE Int. J. Conf. Artificial Intelligence, pages 1035-1040, 1989.

[55] L.S. Homem de Mello and A.C. Sanderson. AND/OR Graph Representation of Assembly Plans. IEEE Transactions on Robotics and Automation, 6(2):188-199, 1990.

[56] D.S. Hong and H.S. Cho. A Neural-network-based Computational Scheme for Generating Optimized Robotic Assembly Sequences. Engineering Applications of Artificial Intelligence, 8(2):129-145, 1995.

[57] J.E. Hopcroft and J.D Ullman. Introduction to Automata Theory, Languages and Computation. Addison-Wesley Publishing Company, Reading, MA, 1979.

[58] W. Hsu, J. Fuh, and Y. Zhang. Synthesis of design concepts from a design for assembly perspective. Computer Integrated Manufacturing Systems, 11(1-2):1-13, 1998.

[59] J.A. Hurt. A Taxonomy of CAD/CAE Systems. Manufacturing Review, 2:170-178, 1989.

[60] K. Ikeuchi, M. Kawade, and T. Suehiro. Assembly Task Recognition with Planar, Curved and Mechanical Contatc. In Proc. IEEE Int. Conf. Robotics and Automation, pages 688-694, 1993. 
[61] K. Ikeuchi and T. Suehiro. Towards an Assembly Plan from Observation. In Proc. IEEE Int. Conf. Robotics and Automation, pages 2171-2177, 1992.

[62] K. Ikeuchi and T. Suehiro. Towards an Assembly Plan from Observation Part I: Task Recognition with Polyhedral Objects . IEEE Transactions on Robotics and Automation, 10(3):368-385, 1994.

[63] R.E. Jones and R.H. Wilson. A Survey of Constraints in Automated Assembly Planning. In Proc. IEEE Int. Conf. Robotics and Automation, pages 1525-1532, 1996.

[64] B. Jung, M. Hoffhenke, and I. Wachsmuth. Virtual Assembly with Construction Kits. In Proc. ASME Design for Engineering Technical Conferences, 1998.

[65] L.E. Kavraki and M.N. Kolountzakis. Partitioning a planar assembly into two connected parts is NP-complete. Information Processing Letters, 55:159-65, 1995.

[66] K.W. Khawaja, A.A. Maciejewski, D. Tretter, and C. Bouman. A Multiscale Assembly Inspection Algorithm. IEEE Robotics $\mathcal{G}$ Automation Magazine, 3(2):15$22,1996$.

[67] S. Kronenberg and F. Kummert. Syntax coordination: Interaction of discourse and extrapositions. In ICSLP, volume 5, pages 2071-2074, 1998.

[68] S. Kronenberg and F. Kummert. Soft unification: Towards robust parsing of spontanous speech. In IASTED Int. Conf. on Artificial Intelligence and Soft Computing, pages 381-385, 1999.

[69] S. Kronenberg and F. Kummert. Structural dependencies between syntactic relations: A robust parsing model for extrapositions in spontaneous speech. In ASRU: IEEE Int. Workshop on Automatic Speech Recognition and Understanding, [Online], 1999. available at: http://asru99.research.att.com/abstracts/4_9297.html.

[70] J.-P. Kruth, G. Van Zeir, and J. Detand. Extracting process planning information from various wire frame and feature based CAD systems. Computers in Industry, 30(2):145-162, 1996.

[71] F. Kummert. Flexible Steuerung eines sprachverarbeitenden Systems mit homogener Wissensbasis. Number 12 in DISKI. infix, Sankt Augustin, 1992.

[72] F. Kummert, G.A. Fink, and G. Sagerer. Schritthaltende hybride Objektdetektion. In E. Paulus and F.M.Wahl, editors, Mustererkennung 97, 19. DAGM-Symposium Braunschweig, Informatik-Fachberichte, pages 137-144. Springer-Verlag, Berlin, 1997. 
[73] F. Kummert, G.A. Fink, G. Sagerer, and E. Braun. Hybrid Object Recognition in Image Sequences. In 14th International Conference on Pattern Recognition, volume II, pages 1165-1170, Brisbane, 1998.

[74] L. Laperrière and H.A. ElMaraghy. GAPP: A Generative Assembly Process Planner. Journal of Manufacturing Systems, 15(4):282-293, 1996.

[75] S. Lee and F.-C. Wang. Physical Reasoning of Interconnection Forces foe Efficient Assembly Planning. In Proc. IEEE Int. Conf. Robotics and Automation, pages 307-313, 1993.

[76] R. Linn and H. Liu. An automatic assembly liaison extraction method and assembly liaison model. IIE Transactions, 31(4):353-363, 1999.

[77] C. L. Liu. Elements of Discrtete Mathematics. McGraw-Hill, 1977.

[78] J.E. Lloyd, J.S. Beis, D.K. Pai, and D.G. Lowe. Programming Contact Tasks Using a Reality-Based Virtual Environment Integrated with Vision. IEEE Transactions on Robotics and Automation, 15(3):423-434, 1999.

[79] B. Luo and E.R. Hancock. Alignment and Correspondence Using Singular Value Decomposition. In Advances in Pattern Recognition, Lecture Notes in Computer Science 1876, pages 226-235. Springer-Verlag, 2000.

[80] E.H. Mamdani and S. Assilian. An Experiment in Linguistic Synthesis with a Fuzzy Controller. Int. Journal of Man Machine Studies, 7:1-13, 1975.

[81] K. Mehlhorn and S. Näher. LEDA: a platform for combinatorial and geometric computing. Cambridge University Press, Cambridge, 1999.

[82] B.T Messmer. Efficient Graph Matching algorithms for preprocessed model graphs. PhD thesis, University of Berne, 1995.

[83] B.T. Messmer and H. Bunke. A New Algorithm for Error-Tolerant Subgraph Isomorphism Detection. IEEE Transactions on Pattern Analysis and Machine Intelligence, 20(5):493-504, 1998.

[84] E. Michaelsen. Über Koordinaten Grammatiken zur Bildverarbeitung und Szenenanalyse. PhD thesis, University of Erlangen-Nürnberg, 1998.

[85] J. Miura and K. Ikeuchi. Task Oriented Generation of Visual Sensing Strategies in Assembly Tasks. IEEE Transactions on Pattern Analysis and Machine Intelligence, 20(2):126-138, 1998.

[86] J. Miura and K. Ikeuchi. Task Planning of Assembly of Flexible Objects and Vision-Based Verification. Robotica, 16:297-307, 1998. 
[87] M. Nagel. Graph-Grammatiken. Vieweg, Braunschweig, 1997.

[88] B. K. Natarajan. On planning assemblies. In Proc. ACM Syposium on Computational Geometry, pages 299-308, 1988.

[89] B.J. Nelson, N.P. Papanikolopoulos, and P.K. Khosla. Robotic Visual Servoing and Robotic Assembly Tasks. IEEE Robotics 83 Automation Magazine, 3(2):2331, 1996.

[90] B. Neumann. Configuration Expert Systems: a Case Study and Tutorial. In H. Bunke, editor, Artificial Intelligence in Manufacturing, Assembly, and Robotics, pages 27-68. Oldenbourg, München, 1988.

[91] J.L. Nevins and D.E. Whitney. Assembly Research. Automatica, 16:595-613, 1980.

[92] N.J. Nilsson. Principles of Artificial Intelligence. Morgan Kaufmann, Los Altos, CA, 1980.

[93] J.A. Noble. From inspection to process understanding and monitoring: a view on computer vision in manufacturing. Image and Vision Computing, 13(3):197-214, 1995.

[94] P. Olivier and J. Tsujii. Quantitative Perceptual Representation of Prepositional Semantics. In P. McKevitt, editor, Intergration of Natural Language and Vision Processing. Kluwer Academic Publishers, 1995.

[95] E. Ostrosi, A. Coulibaly, B. Mutel, and P. Lutz. A PLEX Grammar Approach Directed to Manufacturing Feature Recognition. International Journal of Robotics and Automation, 13(2):33-42, 1998.

[96] B. Parhami. Voting Algorithms. IEEE Transactions on Reliability, 43(4):617-629, 1994.

[97] T. Pavlidis. Structural Pattern Recognition. Springer-Verlag, Berlin, 1977.

[98] J. Pearl. Heuristics. Artificial Intelligence Series. Addison-Wesley, 1984.

[99] W.-H. Qian and E. Pagello. On the Scenario and Heuristics of Disassemblies. In Proc. IEEE Int. Conf. Robotics and Automation, pages 264-271, 1994.

[100] M. Rabemanantsoa and S. Pierre. An artificial intelligence approach for generating assembly sequences in CAD/CAM. Artificial Intelligence in Engineering, 10:97107, 1996.

[101] V. Raghavan, J. Molineros, and R. Sharma. Interactive Evaluation of Assembly Sequences Using Augmented Reality. IEEE Transactions on Robotics and Automation, 15(3):435-449, 1999. 
[102] C. Ramos, J. Rocha, and Z. Vale. On the complexity of precedence graphs for assembly and task planning. Computers In Industry, 36(1-2):101-111, 1998.

[103] M. Ranta, M. Mäntylä, Y. Umeda, and T. Tomiyama. Integration of functional and feature-based product modelling - the IMS/GNOSIS experience. ComputerAided Design, 28(5):371-381, 1996.

[104] A.A.G. Requicha. Representations for rigid solids: Theory, methods, and systems. ACM Computing Surveys, 12(4):437-464, 1980.

[105] A.A.G. Requicha and T.W. Whalen. Representations for Assemblies. In L.S. Homem de Mello and S. Lee, editors, Computer-aided mechanical assembly planning, pages 15-39. Kluwer Academic Press, 1991.

[106] G. Rickheit and I. Wachsmuth. Collaborative Research Centre "Situated Artificial Communicators" at the University of Bielefeld. Artificial Intelligence Review, 10(34):165-170, 1996.

[107] H. Rieser and J. Meyer-Fujara. Zur Semantik von Repräsentationsrelationen. Fallstudie eins zum SFB-Flugzeug. Report 7, SFB 360 'Situierte Künstliche Kommunikatoren', Universität Bielefeld, 1997.

[108] H. Rieser and J. Meyer-Fujara. Aggregates and Depictional Metonymy. In J. Meyer-Fujara and H. Rieser, editors, Proc. Bi-Metonymy, SFB 360 'Situierte Künstliche Kommunikatoren', Universität Bielefeld, 2000.

[109] F. Röhrdanz, H. Mosemann, and F.M. Wahl. Geometrical and Physical Reasoning for Stable Assembly Sequence Planning. In W. Strasse, R. Klein, and R. Rau, editors, Geometric Modelling: Theory and Practice, pages 416-434. Springer-Verlag, Berlin, 1997.

[110] D. Roy. Grounded Speech Communication. In Proc. Int. Conf. on Spoken Language Processing (ICSLP'2000), volume IV, pages 69-72, 2000.

[111] O.E. Ruiz and P.M. Ferreira. Algebraic geometry and group theory in geometric constraint satisfaction for computer-aided design and assembly planning. IIE Transactions, 28(4):281-294, 1996.

[112] G. Sagerer, C. Bauckhage, E. Braun, G. Heidemann, F. Kummert, H. Ritter, and D. Schlüter. Integrating recognition paradigms in a multiple-path architecture. In S. Singh, N. Murshed, and W. Kropatsch, editors, Proc. ICAPR'2001, pages 202-211. Springer-Verlag, 2001.

[113] G. Sagerer and H. Niemann. Semantic Networks for Understanding Scenes. Plenum Publishing Corporation, New York, 1997. 
[114] R. Scha and L. Polanyi. An augmented context free grammar for discourse. In Proc. 12th Int. Conf. of the Association for Computational Linguistics, pages 573-577, 1988.

[115] D. Schlüter, F. Kummert, G. Sagerer, and S. Posch. Integration of regions and contours for object recognition. In Proc. 15th Int. Conf. on Pattern Recognition, pages $944-947,2000$.

[116] D. Schlüter, S. Wachsmuth, G. Sagerer, and S. Posch. Towards an integrated framework for contour-based grouping and object recognition using markov random fiels. In Proc. IEEE Int. Conf. on Image Processing, volume II, pages 100-103, 2000 .

[117] C. Schmied, R. Mohr, and C. Bauckhage. Evaluation of Interest Point Detectors. International Journal of Computer Vision, 37(2):151-172, 2000.

[118] K.T. Seow and R. Devanathan. A Temporal Framework for Assembly Sequence Representation and Analysis. IEEE Transactions on Robotics and Automation, 10(2):220-229, 1994.

[119] L.G. Shapiro and R.M. Haralick. Structural Descriptions and Inexact Matching. IEEE Transactions on Pattern Analysis and Machine Intelligence, 3:504-519, 1981.

[120] M. Sonka, V. Hlavac, and R. Boyle. Image Processing, Analysis and Machine Vision. Chapman \& Hall, London, 1993.

[121] P.C. Stadzisz and J.M. Henrioud. An integrated approach for the design of multiple-product assembly systems. Computers in Industry, 36:21-29, 1998.

[122] J.E. Stoy. Denotational Semantics: The Scott-Strachey Approach to Programming Language Theory. MIT Press, Cambridge, MA, 1977.

[123] T. Suzuki, T. Kanehara, A. Inaba, and S. Okuma. On Algebraic and Graph Structural Properties of Assembly Petri Net. In Proc. IEEE Int. Conf. Robotics and Automation, pages 507-514, 1993.

[124] A. Syed, H.A. ElMaraghy, and N. Chagneux. Real-Time Monitoring and Diagnosing of Robotic Assembly with Self-Organizing Neural Maps. In Proc. IEEE Int. Conf. Robotics and Automation, pages 188-195, 1993.

[125] J.P Thomas and N. Nissanke. An algebra for modelling assembly tasks. Mathematics And Computers In Simulation, 41:639-659, 1996.

[126] J.P. Thomas, N. Nissanke, and K.D Baker. A Hierarchical Petri Net Framework for the Representation and Analysis of Assembly. IEEE Transactions on Robotics and Automation, 12:268-279, 1996. 
[127] J.P. Thomas, N. Nissanke, and K.D Baker. Boundary Models for Assembly Knowledge Representation. IEEE Transactions on Robotics and Automation, 12(2):302$312,1996$.

[128] J.K. Tsotsos, G. Verghese, S. Dickinson, M. Jenkin, A. Jepson, E. Milios, F. Nuflo, S. Stevenson, M. Black, D. Metaxas, S. Culhane, Y. Ye, and R. Mann. PLAYBOT: A visually-guided robot to assist physically disabled children in play. Image and Vision Computing, 16:275-292, 1998.

[129] C.-P. Tung and A.C. Kak. Integrating Sensing, Task Planning and Execution for Robotic Assembly. IEEE Transactions on Robotics and Automation, 12(2):187201, 1996.

[130] W. van Holland, W.F. Bronsvoort, and F.W. Jansen. Feature Modelling for Assembly. In W. Straßer and F.M. Wahl, editors, Graphics and Robotics, pages 131-148. Springer-Verlag, Berlin, 1995.

[131] G. Vosselman. Relational Matching, volume 628 of Lecture Notes in Computer Science. Springer-Verlag, Berlin, 1992.

[132] I. Wachsmuth and B. Jung. Dynamic Conceptualization in a Mechanical-Object Assembly Environment. Artificial Intelligence Review, 10(3-4):345-368, 1996.

[133] S. Wachsmuth. Multi-modal Scene Understanding Using Probabilistic Models. $\mathrm{PhD}$ thesis, Bielefeld University, 2001.

[134] Bonnie Lynn Webber. Structure and ostension in the interpretation of discourse deixis. Language and Cognition Processes, pages 107-135, 1991.

[135] J.D. Wolter. Assembly Sequence Planning Terminology. WWW-Document. available at: http://www.cs.tamu.edu/research/robotics/ Wolter/asp/terms.html.

[136] J.D. Wolter. A Combinatorial Analysis of Enumerative Data Structures for Assembly Planning. Journal of Design and Manufacturing, 2(2):93-104, 1992. 


\section{Index}

AND/OR graph, 16-18, 32, 72-75

AND link, 16

OR link, 16

solution, 16

tree, 16

Assembly

definition, 12-13

function of parts, 27

high-level sequence plan, 53

linear, 48

nonlinear, 48

partial, 13

sequential, 134

task, 13

tree, 46

current, 46

incoming, 46

two handed, 134

CFG, see Context free grammar

Chain, 51, 72, 150

maximal, 51

Conjoin operator, 46-47

Conjoined relation, 45, 72

Context free grammar, 30-31, 145

generator, 39

Cooperative assembly

aspects of, 5

scenario, 3

Correspondence problem, 10

Degree of assemblage, $\mathbf{6 1}$

Difficulty of a task, 119

Discourse, 44

Disparity, 99

Distance between graph labels, 91

between interest points, 109

Haussdorff, 110, 112

subgraph, see Subgraph

ERNEST , 41-42, 141, 145

attribute, $\mathbf{4 2}, 46,49,53,65,67$

concept, 41

instance, 41, 141-144

judgment, 42

link

concrete, 42

part, 41,145

specialization, 41

modality, 41, 145

modified concept, 41, 141-144

relation, 42

Graph

automorphism, 98

edit distance, 89

edit operation, 89

isomorphism, $8 \mathbf{8}$

labeled, $\mathbf{7 7}$

Hypergraph, 16

hyperlink, 16

Interest point, 108

Liaison graph, 15-16, 101

Mating feature, 14, 27, 35, 78-81, 133136

virtual, 66,81

Mating feature graph, 78-81

Mechanical assembly, see Assembly 
Metonymy, 18

depictional, 18

Miscellaneous object, 28

Multiset, 110, 145

intersection, 110

Parser

$\mathrm{LR}(k), \mathbf{4 0}, 46$

reduce action, 46

shift action, 46

Parsing, see Parser

Partially ordered set, 50-51, 148

complete, 83

maximal element, 51

minimal element, 51

Relational description, $\mathbf{7 5}$

Relational model, see Relational description

Semantic domain, $\mathbf{8 3}$

Semantics, 81

denotational, 81

of assembly plan, 83-87, 151-155

SFB 360, 3

Subassembly, 13

Subgraph, $7 \mathbf{7}$

distance, 90

isomorphism, 88, 92, 98

error-correcting, $\mathbf{8 9}$

Visual memory, 106-107 